\title{
Conical Refraction: Fundamentals and Applications
}

\author{
Alejandro Turpin Avilés
}

Submitted in fulfillment of

the requirements for the degree of

Doctor of Physics

Thesis supervisor:

Dr. Jordi Mompart

Departament de Física

Universitat Autònoma de Barcelona

Bellaterra, September 2015 

A mis padres, hermanos, sobrinos y a Raquel 

Tot té un principi i un final i aquest serà el punt final a una etapa de la meva vida la qual estic segur que d'aquí uns anys, quan miri enrere, veuré que ha estat probablement la que ha tingut una major rellevància tant a nivell personal com científic. Quan vaig acabar la carrera no tenia gaire clar quin camí seguir però vaig tenir la sort de parlar amb qui ha estat el meu director de tesi, el Jordi, qui em va guiar i va posar tota la seva confiança en mi des del primer moment. Em va oferir un tema original i es va aventurar a dirigir una tesi amb una forta vessant experimental, tot un atreviment venint d'un teòric! Quan vaig començar el Màster, si m'haguessin dit on he arribat 5 anys després mai m'ho hauria cregut i, Jordi, tu tens gran part de culpa de tot això. Moltes gràcies per haver confiat en mi des del primer dia, per haver-te mostrat sempre tan orgullós de tot el que he fet, per haver estat sempre disponible i per ajudar-me i animar-me quan he passat moments durs durant aquest anys. M'has ensenyat a ser un bon científic, a moure'm dins aquest món, a creure em mi i a ser una millor persona. Mai t'estaré prou agraït per tot això i per haver sigut tan proper amb els teus cotilleos, converses de futbol, política, noies, etc. dinant al bar. Has estat com un pare per a mi (bueno, un germà gran, que tampoc ets tan vell per a ser Mompare :P). Si el Jordi ha estat el meu pare en la ciència, el Yury ha estat el meu gurú científic. Mai he trobat una persona tan pacient, que sàpiga tant de qualsevol tema i amb tanta dedicació com tu. M'has ensenyat a ser riguròs, a tenir un mètode, la importància de estar al dia de tot el que es fa en el cap d'estudi, a programar, i un llarg etcètera. En resum, m'has ajudat a donar-me forma a mi mateix com a científic i a tenir perseverança en el que faig. Tot això sense oblidar que per a mi ets un gran amic! Seguidament, també he donar les gràcies al Todor, una de les persones més enèrgiques que conec i amb més passió per la ciència. Tu vas iniciar tot el tema de la refracció cònica al nostre grup i has demostrat que ets realment qui més coneix de cristalls i del fenomen en si. Si no hagués estat per tu, no podríem haver començat res d'això i no podríem haver explotat el fenomen ni una quarta part del que hem fet. Ets un exemple per tenir sempre un somriure a la cara i per aquesta 
força que et fa tirar endavant tots els teus projectes. També vull donar les gràcies a altres companys del Grup d'Òptica, començant per la Verònica, que va passar de ser una "professora de la carrera" a ser el meu ideal de constància i l'esforç. Ets un exemple professional a més a més d'una persona molt més propera i alegra del que pot semblar a primera vista. A Ramón también le tengo que agradecer su ayuda y su disponibilidad a lo largo de estos años, su paciencia cuando ha tenido que soportar mis imitaciones y su alegría y naturalidad en todo momento. Amb el Francesc també he compartit molts bons moments, sobretot a les diverses activitats de divulgació que hem realitzat junts (tot i que sempre m'ha fet estar alerta perquè cada pregunta seva semblava un examen per a mi). Del Gaspar m'enduc la seva passió com a docent i la seva espectacularitat explicant la ciència, saltant més que els àtoms en inversió de població! També he d'agrair de tot cor als qui han estat els meus companys de doctorat, que han fet que tot sigui molt més fàcil. Primerament, el Menxi, que va passar de ser un simple company a un dels meus millors amics gràcies a las piernas de Madonna, a les nostres apostes i bromes i, en particular, a tot el recolzament que m'ha donat quan pitjor ho he passat. Després, al Bensi, un gran amic també que sempre s'ha mostrat disponible per a ajudar-me a nivell científic i per a treure'm un somriure en tot moment. Es troben a faltar molt les teves frikades! El Dani amb els seus transgordos, bonrollisme constant i que ens va alegrar la vida amb el seu bodorrio!; Shubi with whom I had very funny moments during the master and after that (and extremely good moonwalker); el Juanlu (AKA el Poeta cuántico), que nos ha animado las comidas con sus aventuras y desventuras y a quien valoro muchísimo por haber sido capaz de volver a la carrera científica después de un parón tan largo; i el Joan, amb qui a poc a poc he anat intimant i qui també m'ha ensenyat molt en ciència $i$ ha hagut de soportar les meves frases dramàtiques i fatídiques. També vull donar les gràcies a la Sònia, que sempre que ve pel grup porta el seu aire fresc i la seva alegria y a Irati, por tu paciencia con mis particulitas de las narices y los buenos ratos que hemos pasado en el máster y durante estos años. I no em vull oblidar dels clàssics, que al final han passat de ser "los otros" a ser col-laboradors habituals: el Juan, l'Àngel (la positivitat personificada), l'Alba (una gran companya de salons de l'ensenyament) i la Irene (o Ireniña, campeona de 100 metros lisos pasillo!).

Fora de l'esfera UAB també he trobat grans persones que m'han ajudat a créixer com a científic. El Jose i la Crina han estat sempre uns grans mestres per a mi i sempre han posat a la meva disposició el seu laboratori i coneixements per a ajudar-me en tot el que fos necessari, a més a més de convidar-me en múltiples ocasions a les divertides calçotades que organitzen cada any. I also would like to acknowledge the people from the ANU, in Canberra: Vlad, Cyril, Yan and, in particular, to Wieslaw. You have always been extremely supportive with me, you were very kind with me during my stay in Australia and helped me a lot when needed it at most. També he de donar les gràcies al Gabriel i als Xavis per la seva ajuda i atenció durant els dies que vaig estar a Sidney (tot i que esmorzar chilli no està fet per a mi, Xavi!). Auch, ich möchte die Leute in Darmstadt danke: Sascha (Ich freue mich auf ihren besuch auf Konferenzen), Seppo, 
Alex, Malte (danke für das Fahrrad), Johnny (das Ironman), Felix (immer geduldig und freundlich), Gabriele und Gerhard. Ich habe viele schönes Erinnerungen an meine Zeit in Darmstadt und ich habe viel gelernt von dir. Insbesondere vielen dank Gerhard für deines Unterstützung und Meisterschaft mit mich (I hope that I did not say anything strange...). It was also really nice to go to Glasgow to work in such an excellent environment at the Optics Group and to meet such a great people: Sara (still helping me to find some inaccessible papers!), Reuben, Matt, Dave (great pints-time!), Neal, Tom, Lena, Peter, Emma, Rebecca, Graham (the lord of the lab) and the chinese clan: Yiwei, Minjie, Baoquing and Ray (great Chinese teachers!). In particular, I would like to thank Daniel and Jacqui for their support and help both in the lab and with theoretical issues and for their constant good vibes; and Miles for his guidance and availability. It is very valuable for me that you gave me freedom to develop my own project while guided me to make it as most interesting as possible.

Moltes vegades concentrar els nostres agraïments en gent que ens ha ajudat a desenvolupar la carrera científica de manera més directa, com pot ser un director de tesi o col-laboradors, però crec que hi ha d'altres persones sense les quals no podria haver aconseguit fer aquesta tesi. En este sentido quiero dar las gracias a mis profesores del instituto IES Ferran Casablancas y, en particular, a M $\mathrm{M}^{\mathrm{a}}$ José y Moisés. Entre los dos pusisteis la semilla de todo lo que he sido académicamente hasta el momento. Por un lado, Ma José me enseñó a dar el máximo de mí, a exigirme todo lo que pudiera exigirme para ser yo mismo mejor persona y, aunque quizá no lo supe valorar suficiente cuando tenía 15 años, ahora tengo bien claro que lo que he conseguido ha sido en buena medida a ese cambio de chip que me hiciste tener. También recuerdo constantemente lo que me escribiste en aquella actividad de tutoría y espero que con el tiempo siga dándote la razón. Por otro lado, Moisés despertó en mí algo que más adelante descubrí que había llevado latente toda mi vida: la pasión por la física y por conocer cómo funciona el mundo que nos rodea. Hiciste que tuviera claro que quería estudiar física, porque necesitaba saber más de todo lo que nos habías ido transmitiendo con esa pasión y perseverancia. Siempre os lo agradeceré. I també m'agradaria fer una menció al Josep Puigdomenech, un gran professor de matemàtiques de qui sempre guardaré una frase al meu cap: entendre és un plaer molt fi. No hi puc estar més d'acord!

Ara toca el torn dels amics. Primero quiero agradecer a los amigos de toda la vida: Floren, mi primer amigo (aunque no me gustase que me llamaras tío) y uno de los más auténticos que tengo, que siempre se ha mostrado interesado por lo que hago a la par que orgulloso (como ves, al final sí que he sido inventor, como dijimos cuando éramos pequeños y menos mal que me dio por eso y no por asesino a sueldo! :P); Rubeniki (no digo la frase que sigue), que fue un gran descubrimiento y cuyas birras semanales me han ayudado a desconectar del trabajo; Rubenxo, con quien comparto pasión por el trabajo y aún más por la comida; a Linares el padre del año, que tantas risas y buenos momentos me ha hecho pasar y a Carlos, que fue un gran apoyo durante mucho tiempo. També estic molt agraït per a haver trobat grans persones que van començar 
sent companys de carrera i han acabat sent uns amics genials: el Jordi, que sempre s'ha interessat per mi quan ho he necessitat, amb qui ens hem recolzat mútuament durant la carrera i el doctorat i a qui admiro (tot i que no et perdono totes les $\mathrm{MH}$ que em vas treure!); el Parra, que fue quien más estuvo ahí cuando peor lo pasé y se ha mostrado siempre interesado y orgulloso por lo que hago; y Juanfra ( $\sin n$ ), una de las personas con quien mejor me lo paso y de la cual valoro mucho que siempre diga lo que piensa. También quiero dar las gracias a la gente del Balonmano, que también siempre demuestran interés por todo lo que hago y con quien paso muy buenos ratos por las tardes: Sergi y Antchonio (dos grandes maestros), Raul, Xavi, Marcos, Rober, Loren y Hippy.

Otra persona que siento que ha sido importantísima para esta tesis ha sido Raquel, aunque no haya estado desde el día que la empecé. No me has ayudado a desarrollar ninguna investigación, ni académicamente, pero me has apoyado y siempre has sabido escuchar mis inquietudes y problemas desde el primer día (y soy consciente que puedo ser un tostón :P). Cuando me he ido fuera y he estado solo, has estado ahí para que me sienta tranquilo y has podido aguantar lo que significa tener una pareja que le dedica el $80 \%$ de su tiempo a su trabajo, incluyendo viajes y estancias que en total suman una buena parte del tiempo que llevamos juntos. Muchas gracias por hacerme la vida más fácil y por hacerme feliz, no dudes que eso me ha ayudado y mucho para conseguir todo esto. Su familia también ha sido un gran apoyo para mí e incluso a veces se han mostrado interesados por lo que hago, cosa que sé que no es fácil! Por eso, muchas gracias Salva, Mariasun, Asunción, Sandra, Alex, David, Ester y Paula (valiente reto tuve con vosotras dos!).

Finalmente, no tengo suficientes palabras para agradecer a mi familia todo lo que han hecho por mí. Mis padres, Enrique y Victoria han sido geniales durante toda mi vida y me han dado fuerzas especialmente durante estos últimos años. De mi madre he aprendido a ser un luchador, a tener una sonrisa en la cara siempre y a ver las cosas por el lado positivo. La verdad es que nunca he conocido a alguien tan fuerte, enérgico y alegre como tú y es un honor para mí ser tu hijo. De tu boca siempre he encontrado las palabras tú tienes que hacer lo que te haga más feliz, siempre me has apoyado en todo y nunca me has reprochado nada. Eres la mejor madre que podría tener. Y mi padre es un crack, una de esas personas que marcan a quien lo conoce. Eres luchador y rebelde como nadie y me encanta haber sacado esas virtudes de ti, aunque a veces se nos vayan de las manos. Siempre te has preocupado para que no me falte de nada $y$, mientras haya estado en tu mano, nunca has dejado que hubiera ninguna injusticia conmigo. Todo eso sin olvidar la de veces que me haces reir, aunque no sea ese tu propósito. Espero que con esta tesis os devuelva a los dos un poquito de todo lo que me habéis dado y podáis estar orgullosos de mí (pero papa no lleves la tesis en el bolsillo por favor, que te conozco!). Mis hermanos, también siempre me han dado todo su apoyo y se lo agradeceré siempre. Charly, he pasado la mayoría del tiempo contigo y en todas mis elecciones siempre me has dado tu opinión y tu apoyo cuando has visto que tenía 
algo claro. Tu manera de ser siempre ha sido una referencia para mí y me gusta ver que en muchas cosas somos parecidos. Enrique, a nivel profesional siempre has sido una gran referencia, siempre ha sido un orgullo que la gente te conociera por lo que haces y me lo comentaran. Espero que sientas parte de eso tú con esta tesis (que no es por restregar nada, pero al final seré doctor antes que tú! :P). También quiero darles las gracias mis sobrinos Martina y Mateu. Martina, ya estás hecha una mujercita, como pasa el tiempo! Espero que te sientas tan orgullosa de tu tito como cuando de pequeña le explicabas a tus profes las cosas que yo te contaba del universo y los dejabas con la boca abierta. Ser tu tío siempre me ha hecho muy feliz. Mateu, encara ets petit per a poder llegir tot això però no t'imagines com d'orgullós em sento de que tinguis la curiositat del teu tiet i les ganes de saber el perquè de tot. Algun dia farem un robot junts! Gràcies per impregnar-me de l'alegria i la vida que portes a casa cada cop que vens. Y para acabar, quiero también agradecer a mis tíos Antonio y Alicia, por ser unas personas tan generosas y buenas, siempre dispuestas a ayudar en todo momento y muy interesadas por mis preocupaciones en todos los sentidos.

Els qui millor em coneixen ja saben el que us diré a continuació: sóc un despiste, així que si em deixo a algú als agraïments no m'ho tingueu en compte.

La realización de esta beca ha sido posible gracias a una beca de Formación del Profesorado Universitario del MECD (Refs. AP2010-2310 y Est13/00538) und dank eines Stipendiums von der Deutscher Akademischer Austauschdienst wann war ich in Darmstadt für drei Monate (Ref. 91526836). Per tant, dec 0.11 cèntims a cada contribuent a Espanya und 0.004 Cent für jede Deutsch. Recordeu-me que us ho torni :)

Alejandro Turpin Avilés

Bellaterra, Setembre de 2015. 

Conical refraction is a phenomenon occurring in biaxial crystals that was predicted theoretically by Hamilton in 1832 observed by Lloyd few months later. During many years, this optical effect was considered as a curiosity and almost fell into oblivion until the last quarter of the $20^{\text {th }}$ century. One of the reasons for this is that crystal technology was not developed enough to offer high quality biaxial crystals cut perpendicular to one of the optic axes. This issue has been solved during the last decades and with the start of the $21^{\text {st }}$ the community started studying the conical refraction phenomenon both experimentally and theoretically. There are three main reasons that make conical refraction so attractive:

1) When using a focused Gaussian input beam the conical refraction beam forms an optical bottle, i.e. it opens and closes itself symmetrically along its propagation leaving a central region without intensity.

2) At the intermediate region of the optical bottle -the focal plane-, the transverse intensity pattern is formed by a pair of concentric bright rings split by an exactly null dark ring known as Poggendorff ring.

3) The state of polarization of the rings is linear and unique at every point in a way such that every two diametrically opposite points of the ring have orthogonal polarizations. This polarization distribution differs from the well known radial and azimuthal polarization distributions.

Inspired by these features, with the present thesis we have the aim of analyzing in detail the phenomenon of conical refraction and to exploit it in different areas of science and technology. The organization of the thesis does not follow the temporal line of development of our work but we have tried to find the most constructive way to understand the conical refraction phenomenology and its applications.

The thesis can be divided in two main parts in which 1) we analyze in detail the phenomenon of conical refraction (Chapters 18) and 2) we apply it in different fields of 
fundamental science and technology (Chapters 6610). Unless otherwise stated, all the work presented here has been performed in the Conical Refraction Laboratory at the Optics Group of the Universitat Autònoma de Barcelona in collaboration with Dr. Yury V. Loiko, Dr. Todor K. Kalkandjiev and Prof. Jordi Mompart.

Chapter 1 is devoted to introduce conical refraction historically: from Hamilton's prediction and the first experiments from Lloyd to the diffractive theory of Belsky, Khapalyuk and Berry and the last reported experiments. We also give a brief introduction to crystal optics in order to understand the fundamental physics of the phenomenon.

Then, in Chapter 2 we present the theory of conical refraction for both a single crystal and cascade of them. We divide the theory in three complementary views: the diffractive theory developed by Belsky and Khapalyuk and reformulated in an elegant way by Berry (Chapter 2.2) and our group, the dual-cone model introduced by Sokolovskii and co-workers and reformulated by our group (Chapter 2.4), and the wavevector and polarization interpretation introduced by our group (Chapter 2.3).

One feature that has not been commented above about the nature of conical refraction is the fact that the beam evolution is governed by the parameter $\rho_{0}$, obtained as the ratio between the ring radius $R_{0}$ and the waist radius of the focused input beam $w_{0}$, i.e., $\rho_{0} \equiv \frac{R_{0}}{w_{0}}$. We show the relevance of $\rho_{0}$ Chapter 3 and demonstrate that, by adjusting appropriately this parameter, there can be obtained very interesting light beams, such as three-dimensional dark focus and a super-Gaussian beam.

Besides, the $\rho_{0}$ parameter not only controls the intensity pattern but also the state of polarization of the conical refraction beam. Beams at which the state of polarization and the intensity pattern do not factorize are known as vector beams. In Chapter 4 we show different vector beams generated with conical refraction and demonstrate that their polarization distribution depends, in general, on the state of polarization of the input beam. We also show that the spin-orbit coupling provided by the biaxial crystal leads to different optical singularities in the conical refraction beam.

The birefringence introduced by the biaxial crystal has the consequence that, even if the input beam lacks of some the wave-vectors (for instance, when the beam is blocked partially by an obstruction), the conical refraction beam can preserve in part its annular structure. The reconstruction of after an obstruction is a process known as self-healing. In Chapter 5 it is demonstrated that conically refracted beams also self-heal on some way. In our approach, we do not obstruct the conical refraction beam itself but we do it with the Gaussian input beam by using an azimuthal obstruction. We show that even when half of the input beam is blocked, the transverse intensity pattern at the focal plane is still ring-like.

Biaxial crystals are mostly used as non-linear media to give rise to non-linear effects such as second harmonic generation, parametric down-conversion, Kerr effect, etc. Second harmonic generation in conical refraction has been studied together, in the same biaxial crystal. The approach that is presented in Chapter $\mathbf{8}$ slightly differs from that 
one. We study the second harmonic generation of a conically refracted beam in type I and type II non-lineal crystals. This arrangement is very efficient, since the phase matching orientation of the non-linear crystal can be adjusted independently on the optic axis of the crystal giving rise to conical refraction. We show the beam evolution of the SHG beams when the non-linear crystal is placed at the ring plane of conical refraction and give a formula for the intensity pattern at the focal plane.

Chapters 6 and 7 are related to one of the major applications of conical refraction: optical trapping. At variance with other works where conical refraction is used as for optical twzeers, our approach takes profit of the force of photophoresis and the dipolar force to trap macroscopic absorbing particles and Bose-Einstein condensates. With photophoresis (Chapter [6), we demonstrate the efficient loading and unloading of macroscopic particles by reconfiguring in real-time the optical bottle provided by the conical refraction beam. This work was thought and carried out during a short research stay that was carried out at the group of Prof. Wieslaw Krolikowski from the Australian National University. For the case of optical trapping with dipole potentials (Chapter 7), we give the trapping frequencies and potential depths for different optical potentials available with conical refraction. For the particular cases of the Poggendorff dark ring and the three-dimensional dark focus we give analytical formulas deduced directly from the theory of conical refraction and we present an experimental implementation of these optical potentials. Most of this research with Bose-Einstein Condensates was developed during a research stay funded by the DAAD at the group of Prof. Gerhard Birkl at the Technische Universität Darmstadt.

Chapter 9 we present the first proposal of combining conical refraction with quantum optics. Firstly, we analyze the transformation of a spontaneous parametric downconversion beam generated in a non-linear crystal, which leads photons entangled in linear momentum. Then, we propose a method to demonstrate such entanglement through a polarization measurement, i.e., we swap linear momentum entanglement into polarization entanglement by means of the conical refraction phenomenon. This proposal was developed during a short research stay that was carried out at the group of Prof. Miles Padgett from the University of Glasgow and funded by the MICINN.

In addition to all the implementations at a fundamental science level, conical refraction also has technological applications, as it is shown in Chapter 10. In particular, we report a free space optical communication system for multiplexing and demultiplexing multiple polarization channels with a cascade of 3 biaxial crystals.

Finally, Chapter 11 gives a summary of the work presented in this thesis and envisages possible future work. 



\section{List of publications by Alejandro Turpin}

The research contained in this thesis can be found in the publications listed below:

\section{Chapter 2: Theory and fundamental experiments on conical refraction}

1. A. Turpin, Yu. V. Loiko, T. K. Kalkandjiev, H. Tomizawa, and J. Mompart, "Wave-vector and polarization dependence of conical refraction," Opt. Express 21, 4503-4511 (2013).

2. A. Turpin, Yu. V. Loiko, T. K. Kalkandjiev, and J. Mompart, "Multiple rings formation in cascaded conical refraction," Opt. Lett. 38, 1455-1457 (2013).

3. A. Turpin, Yu. Loiko, T. K. Kalkandjiev, H. Tomizawa, and J. Mompart, "On the dual-cone nature of the conical refraction phenomenon," Opt. Lett. 40, 1639-1642 (2015).

4. A. Turpin, Yu. V. Loiko, T. K. Kalkandjiev, and J. Mompart, "Light propagation in biaxial crystals," J. Opt. 17, 065603 (2015).

5. A. Turpin, A. Vargas, A. Lizana, F. A. Torres-Ruiz, I. Estévez, I. Moreno, J. Campos, and J. Mompart, "Transformation of vector beams with radial and azimuthal polarizations in biaxial crystals," J. Opt. Soc. Am. A 32, 1012-1016 (2015).

\section{Chapter 3: Beam shaping with conical refraction}

6. Yu. V. Loiko, A. Turpin, T. K. Kalkandjiev, E. U. Rafailov, and J. Mompart, "Generating a three-dimensional dark focus from a single conically refracted light beam," Opt. Lett. 38, 4648-4651 (2013).

7. A. Turpin, Yu. V. Loiko, T. K. Kalkandkiev, H. Tomizawa, and J. Mompart, "SuperGaussian conical refraction beam," Opt. Lett. 39, 4349-4352 (2014).

Chapter 4: Novel vector beams and optical singularities in conical refraction 8. A. Turpin, Yu. V. Loiko, A. Peinado, A. Lizana, T. K. Kalkandjiev, J. Campos, and J. Mompart, "Polarization tailored novel vector beams based on conical refraction," Opt. Express 23, 5704-5715 (2015). 
Chapter 5: Conical refraction healing after partially blocking the input beam

9. A. Turpin, Yu. V. Loiko, T. K. Kalkandjiev, R. Corbalán, and J. Mompart, "Conical refraction healing after partially blocking the input beam," Phys. Rev. A 92, 013802 (2015).

\section{Chapter 6: An optical vault for absorbing particles}

10. A. Turpin, V. Shvedov, C. Hnatovsky, Yu. V. Loiko, J. Mompart, and W. Krolikowski, "Optical vault: A reconfigurable bottle beam based on conical refraction of light," Opt. Express 21, 26335-26340 (2013).

11. A. Turpin and J. Mompart, "Una cámara acorazada óptica," Investigación y ciencia 463, 14-15 (2015).

\section{Chapter 7: Trapping of BEC with conical refraction}

12. A. Turpin, J. Polo, Yu. V. Loiko, J. Küber, F. Schmaltz, T. K. Kalkandjiev, V. Ahufinger, G. Birkl, and J. Mompart, "Blue-detuned optical ring trap for Bose-Einstein condensates based on conical refraction," Opt. Express 23, 1638-1650 (2015).

13. A. Turpin, J. Küber, F. Schmaltz, Yu. V. Loiko, J. Mompart, and G. Birkl, "Trapping of Bose-Einstein condensates in a three-dimensional dark focus generated by conical refraction," submitted to publication.

\section{Chapter 8: Second harmonic generation of a conically refracted beam}

14. A. Turpin, Yu. V. Loiko, T. K. Kalkandjiev, J. Trull, C. Cojocaru, and J. Mompart, "Type I and type II second harmonic generation of conically refracted beams," Opt. Lett. 38, 2484-2486 (2013).

\section{Chapter 10: Conical refraction for free-space optical communications}

15. A. Turpin, Yu. V. Loiko, T. K. Kalkandjiev, and J. Mompart, "Free-space optical polarization demultiplexing and multiplexing by means of conical refraction," Opt. Lett. 37, 4197-4199 (2012).

16. A. Turpin, Yu. V. Loiko, T. K. Kalkandjiev, and J. Mompart, "System, method, transmitter and receptor for optical communications," patent application (EP), P201230105 (2012).

Other publications of Alejandro Turpin not contained in this thesis:

17. A. Peinado, A. Turpin, A. Lizana, E. Fernández, J. Mompart, and J. Campos, "Conical refraction as a tool for polarization metrology," Opt. Lett. 38, 4100-4103 (2013).

18. A. Peinado, A. Turpin, A. Lizana, T. K. Kalkandjiev, J. Mompart, and J. Campos, "Complete polarimeter based on conical refraction," patent application (EP), PCT/EP2014/065918 (2014). 
19. A. Peinado, A. Lizana, A. Turpin, C. Iemmi, T. K. Kalkandjiev, J. Mompart, and J. Campos, "Optimization, tolerance analysis and implementation of a Stokes polarimeter based on the conical refraction phenomenon," Opt. Express 23, 5636-5652 (2015).

20. A. Peinado, A. Turpin, C. Iemmi, A. Márquez, T. K. Kalkandjiev, J. Mompart, and J. Campos, "Interferometric characterization of the structured polarized light beam produced by the conical refraction phenomenon," Opt. Express 23, 18080-18091 (2015).

21. A. Lizana, I. Estévez, A. Turpin, C. Ramirez, A. Peinado, and J. Campos, "Implementation and performance of an in-line incomplete Stokes Polarimeter based on a single biaxial crystal," to appear in Appl. Opt. (2015). 

1 History of conical refraction 1

1.1 The triumph of the wave theorv of light . . . . . . . . . . . . . 1

1.2 Elements of crystal optics . . . . . . . . . . . . . . . . . . . . . . . . . . . . . . .

1.2 .1 Uniaxial crystals $\ldots \ldots \ldots \ldots \ldots$

1.2 .2 Biaxial crystals . . . . . . . . . . . . . . . . . . 7

1.3 The subtle phenomenon of conical refraction . . . . . . . . . . 8

1.4 Everything was not said: new observations on conical refraction . . . . . 10

1.5 From Hamilton's model to the diffractive theory of conical refraction . . 11

1.6 Conical refraction out of crystal optics . . . . . . . . . . . . . . . . 12

2 Theory and fundamental experiments on conical refraction 15

2.1 Introduction . . . . . . . . . . . . . . . . . . . . . 16

2.2 Diffractive solution . . . . . . . . . . . . . . . . . . . . . . 18

$2.2 .1 \quad$ Cylindrically symmetric solution . . . . . . . . . . . . . . 18

2.2 .2 Non-cylindrically symmetric solution . . . . . . . . . . . . . 21

$2.3 \quad$ Wave-vector and polarization description of conical refraction . . . . . . 23

2.3 .1 Conical refraction of spatially anisotropic beams revisited . . . . 24

2.3 .2 Transformation rules of conical refraction . . . . . . . . 26

2.3.3 Application of the transformation rules of $\mathrm{CR}$ to an axicon beam 29

2.4 Dual-cone model of conical refraction . . . . . . . . . . . . . . . 31

2.4.1 Dual-cone theory and its relation to the wave-vector interpretation 31

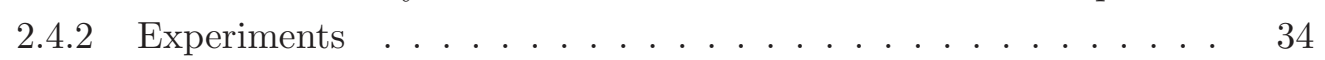

2.5 Cascaded conical refraction . . . . . . . . . . . . . . 38

2.5 .1 Coordinates system and single crystal configuration . . . . . . 39

2.5 .2 Multiple crystals . . . . . . . . . . . . . . . . . . . . . . . 40

2.6 Conclusions $\ldots \ldots \ldots \ldots$ 
$\begin{array}{lll}3 & \text { Beam shaping with conical refraction } & 47\end{array}$

3.1 Introduction . . . . . . . . . . . . . . . . . . . . . . . . . 47

3.2 Generating a 3D dark focus with conical refraction . . . . . . . . . . . . . . 48

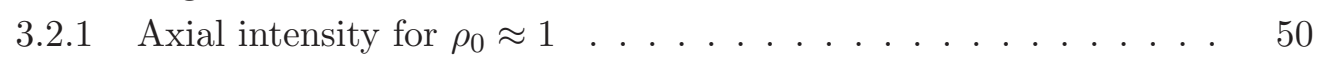

3.2.2 Characteristics of the 3D dark focus beam . . . . . . . . . . . . . 52

3.3 A Super-Gaussian conical refraction beam . . . . . . . . . . . 54

3.3.1 Characteristics of the Super-Gaussian conical refraction beam . . 55

3.4 Conclusions . . . . . . . . . . . . . . . . . 57

4 Novel vector beams and optical singularities in conical refraction 59

4.1 Introduction . . . . . . . . . . . . . . . . . . . . . . . . . . . . . . 60

4.2 Theoretical background . . . . . . . . . . . . . . . . . 60

4.2 .1 Analvsis of the $B_{0}$ and $B_{1}$ functions . . . . . . . . . . 60

4.2 .2 Stokes vector formalism . . . . . . . . . . . . . . . . . . . . . . . . . . . . . 64

4.3 State of polarization for $\rho_{0} \gg 1 \ldots \ldots \ldots$. . . . . . . . . . . 64

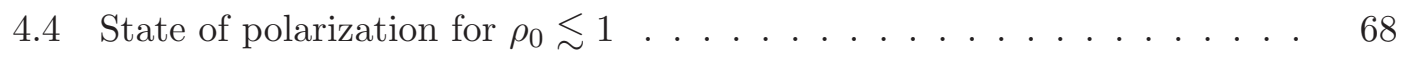

4.5 Discussion in terms of spin-orbit coupling . . . . . . . . . . . . . . . . . . 72

4.6 Conclusions . . . . . . . . . . . . . . . . . . . . . 74

5 Conical refraction healing after partially blocking the input beam $\quad 77$

5.1 Introduction . . . . . . . . . . . . . . . . . . . . . . . 77

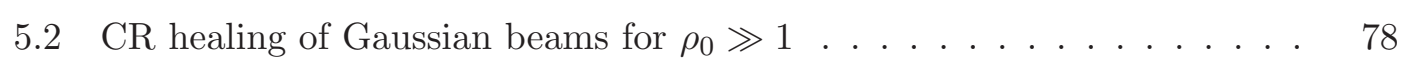

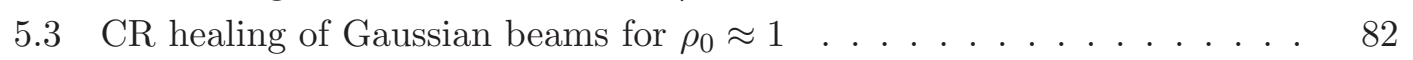

5.4 Conclusions . . . . . . . . . . . . . . . . . . 84

6 An optical vault for absorbing particles $\quad 85$

6.1 Introduction . . . . . . . . . . . . . . . . . . . . . . . . . . . . . . . . . . . . .

6.2 The photophoretic force $\ldots \ldots \ldots \ldots$. . . . . . . . . . . . . . . . . . . . . . 86

6.3 Experimental proposal $\ldots \ldots \ldots \ldots$. . . . . . . . . . . . . . . . . . . . . . . . . . . . . . . . 88

6.4 Experimental results $\ldots \ldots \ldots \ldots$. . . . . . . . . . . . . . . . . . . . . . . . . . . . . . . . . . . . . .

6.5 Conclusions . . . . . . . . . . . . . . . . . . . . . . 92

$7 \quad$ Trapping Bose-einstein condensates with conical refraction 93

7.1 Introduction . . . . . . . . . . . . . . . . . . . . . . . 94

7.2 Trapping BECs in a $3 \mathrm{D}$ dark focus . . . . . . . . . . . . . . . . 94

7.2.1 Theoretical formulation for the 3D dark focus to atom trapping . 95

7.3 Experimental trapping of a ${ }^{87} \mathrm{Rb}$ BEC in the 3D dark focus . . . . . . . 98

7.4 Blue-detuned optical ring traps for BECs based on conical refraction . . 100

7.4.1 Asymptotic solution close to the Poggendorff dark ring . . . . . . 101

7.4 .2 Harmonic potential approximation . . . . . . . . . . . . . 103

7.4.3 Numerical simulations of a BEC of ${ }^{87} \mathrm{Rb}$ atoms . . . . . . . . . 105

7.5 Conclusions . . . . . . . . . . . . . . . . . . . . 108 
8 Second harmonic generation of a conically refracted beam 111

8.1 Introduction . . . . . . . . . . . . . . . . . . . . . . . 112

8.2 Experimental set-up . . . . . . . . . . . . . . . . . . . . . . . . 113

8.3 Transverse intensitv patterns at the focal plane . . . . . . . . . . . . . 114

8.4 Beam evolution of the SHG beams . . . . . . . . . . . . . 116

8.5 Influence of the position of the non-linear crystal . . . . . . . . . . . 117

8.6 Conclusions . . . . . . . . . . . . . . . . . . . . 117

$9 \quad$ Testing quantum mechanics with conical refraction 119

9.1 Introduction . . . . . . . . . . . . . . . . . . . . . . 119

9.2 Conical refraction of a spontaneous parametric down-converted beam . . 121

9.3 Detection of linear momentum entanglement with conical refraction . . 123

9.4 Conclusions . . . . . . . . . . . . . . . . . . . 126

10 Conical refraction for free-space optical communications $\quad 127$

10.1 Introduction . . . . . . . . . . . . . . . . . . . . . 127

10.2 The forward-backward conical refraction transformation . . . . . . . . 128

10.3 Multiplexing and de-multiplexing proposal and experimental results . . 130

10.4 Conclusions . . . . . . . . . . . . . . . . . . 133

$\begin{array}{ll}11 \text { Conclusions and outlook } & 135\end{array}$

\begin{tabular}{lr}
\hline Bibliography & 141
\end{tabular} 

CHAPTER 1

History of conical refraction

This Chapter serves to introduce the phenomenon of Conical Refraction and its meaning to the theory of light. Our aim is to show the historical evolution of the phenomenon, including both the development of the theory of conical refraction and milestone experiments, as long as we introduce briefly some ideas of crystal optics. The organization of the Chapter is the following. In Section 1.1 we show the relevance of the conical refraction phenomenon for the acceptance of the wave nature of light. Then, after a brief introduction of crystal optics given in Section 1.2, we discuss how Hamilton discovered conical refraction in biaxial crystals (Section 1.3), the new observations of the phenomenon reported by Voigt, Poggendorff and Raman (Section 1.4); and show the development of the diffractive theory of conical refraction introduced by Lalor and further developed later by Belsky and Khapalyuk and reformulated by Berry (Section 1.5). Finally, in Section 1.6 we discuss other areas of knowledge in which conical refraction plays a role.

\subsection{The triumph of the wave theory of light}

The prediction of conical refraction (CR) in biaxial crystals by William Rowan Hamilton is closely related to the triumph of the wave theory of optics over its corpuscular picture. At the beginning of the $19^{\text {th }}$ century there was a fight between backers of both theories. However, the corpuscular-minded scientists had some advantage because Sir Isaac Newton, one of the fathers of scientific thought, was one of the major contributors to that theory [1. Among all the known phenomenology related to light by that time, double refraction observed in anisotropic uniaxial crystals was one of the candidates to tip the balance towards one of the two theories. 
The effects of anisotropic media over a light beam are known since the $17^{\text {th }}$ century, when Rasmus Bartholin in 1669 reported the observation of the splitting of an incident light beam into two sub-beams after passing through a plate of the Iceland mineral [2]. Bartholin observed that while one of the two sub-beams refracted following the ordinary Snell's law, the other sub-beam followed a new extraordinary law. This phenomenon is known as double refraction and it is a consequence of the birefringence of the medium generated by an anisotropy in the material structure. Some years after, in 1690, Christiaan Huygens presented a novel description of light propagation in anisotropic media where light is assumed to be a wave that, when passing through a uniaxial crystal, refracts as two internal waves [3]. In Huygens' description, the ordinary wave refracts as a sphere, i.e. following Snell's law in all directions, but the extraordinary wave refracts as an ellipsoid. Both the sphere and the ellipsoid touch each other at only two points and the line that connects these points gives the direction of the optic axis of the material. Along this direction, the input beam into the crystal propagates without splitting at all. This new theory established the roots for the wave theory of light that came in the following centuries. In fact, the wave theory of double refraction was revised in detail during the $19^{\text {th }}$ century by George Gabriel Stokes among others, who could nothing else than verify it.

Although Huygens' theory described well all the double refraction experiments carried out by that time, it was rejected by Newton because the origin of the two orthogonal polarizations in waves was unclear. In addition, David Brewster in 1813 discovered that the mineral topaz has two different directions along which no refraction was observed, i.e. two optic axes. Accordingly, these type of crystals were named biaxial crystals. Further investigations showed that aragonite, borax or mica also behave as biaxial crystals. The theory of Huygens could not explain the behavior of light in these materials, what weaken even more the wave theory of light against the corpuscular theory. In addition, Pierre Simon Laplace and Etienne Louis Malus were able to deduced double refraction effects from a corpuscular point of view.

However, not everything was lost for the wave theory of light. At the beginning of the $19^{\text {th }}$ century, Thomas Young was convinced that light was a wave and in 1801 presented his milestone contribution on the interference of light waves and the slit experiment [4. Nevertheless, the shadow of Newton was too large such that Young's experiment and its wave explanation was not accepted by the community. In 1819, Agustin-Jean Fresnel presented an independent description of the wave theory of light to a competition launched by the Académie des Sciences to explain the properties of light. Fresnel's theory of light included the effects of interference, diffraction and double refraction both in uniaxial and biaxial media [5]. Concretely, in that work, he predicted that a beam of light passing through a pinhole creates an interference intensity pattern such that an on axis maximum intensity spot is found. In contrast, the corpuscular theory of light predicted a dark intensity region at that point. The experimental observation of Dominique-François-Jean Arago (who was the head of the comitee of the Académie des 
Sciences) of Fresnel's prediction was the first big victory of the wave theory of light.

Regarding double refraction, Fresnel's theory states that, in general, a wave of light propagating through a medium does it differently in any of the three Cartesian directions. As a consequence, the wave surface does not form an ellipsoid nor a spheroid but a surface of the $4^{\text {th }}$ order formed by two sheets whose points of contact with the tangent planes at any point give the direction of the two refracted beams in the crystal. Note that, although similar, this new law differs with Snell's law and Huygens' law, in general. If the wave refracts identically in all three Cartesian directions, i.e. if the medium is isotropic, Snell's law governs the propagation of light. If two of the three Cartesian directions of refraction are equal, then the medium is uniaxial, it possesses an optic axis through which no double refraction is observed and Huygens' law can be applied. Finally, if all three directions are different, the medium possesses two optic axes through which light does not double refract, while in any other direction double refraction is observed.

One of the problems of Fresnel's theory of light was that the procedure that he followed to obtain it was involved and inelegant and this did not give the final victory to the wave theory of light. Anyways, the Fresnel wave surface and its properties were studied exhaustively during all the $19^{\text {th }}$ century by scientists such as André Marie Ampère, Augustin Louis Cauchy, Lord Rayleigh and William Rowan Hamilton, who was the culpable of the final triumph of the wave theory of light, as we will discuss in Section 1.3

\subsection{Elements of crystal optics}

In the previous section we have highlighted the relevance of the Fresnel surface for the triumph of the wave theory of light. In what follows section we present the Fresnel surface formally in order to understand CR in that context. We do not follow the procedure developed by Fresnel since we have the possibility of using Maxwell equations, which were established decades after Fresnel's theory. A deeper analysis of crystal optics is presented in Chapter 15 of Ref. [6] and in Chapter 11 of Ref. [7].

We consider the propagation of an electromagnetic monochromatic plane wave through an electrically linear anisotropic medium with permittivity

$$
\hat{\varepsilon}=\left(\begin{array}{ccc}
\epsilon_{x x} & \varepsilon_{x y} & \varepsilon_{x z} \\
\epsilon_{y x} & \varepsilon_{y y} & \varepsilon_{y z} \\
\epsilon_{z x} & \varepsilon_{z y} & \varepsilon_{z z}
\end{array}\right),
$$

where $\varepsilon_{i j}$ are constants, and permeability $\hat{\mu}=\mu_{0} \mathbf{I}$, where $\hat{\mathbf{I}}$ is the identity matrix. The diagonalized form of the permittivity tensor from Eq. (1.1) reads as follows

$$
\hat{\varepsilon}=\left(\begin{array}{ccc}
\varepsilon_{1} & 0 & 0 \\
0 & \varepsilon_{2} & 0 \\
0 & 0 & \varepsilon_{3}
\end{array}\right) .
$$


An electromagnetic field $\mathbf{E}$ applied to the medium forces the charges of the latter to re-organize. This re-organization can be described by means of the displacement vector D through the identity

$$
\mathbf{D}=\hat{\varepsilon} \mathbf{E} .
$$

A similar relation is found for the magnetic field $\mathbf{H}$ and the magnetic inductance $\mathbf{B}$ :

$$
\mathbf{H}=\hat{\mu}^{-1} \mathbf{B} .
$$

Eqs. (1.3) and (1.4) are the constitutive equations of electromagnetism. In order to have complete information of the propagation of an electromagnetic field passing through a medium, Maxwell equations must be also considered:

$$
\begin{aligned}
\nabla \cdot \mathbf{E} & =\rho \\
\nabla \cdot \mathbf{B} & =0 \\
\nabla \times \mathbf{E} & =-\frac{\partial \mathbf{B}}{\partial t}, \\
\nabla \times \mathbf{H} & =\mathbf{J}+\frac{\partial \mathbf{E}}{\partial t} .
\end{aligned}
$$

For a linear dielectric anisotropic medium, there are neither free charges $(\rho=0)$ nor currents $(\mathbf{J}=0)$. The propagating electromagnetic field we consider is a monochromatic transverse plane wave with angular frequency $\omega$ propagating at speed $c / n$ (being $n$ the refractive index) with electric field in the form

$$
\mathbf{E}=E_{0} \exp \left[i \omega\left(\frac{n}{c} \mathbf{k} \cdot \mathbf{r}-t\right)+\delta\right] \mathbf{e}_{\mathbf{0}},
$$

where $E_{0}$ is a constant, $\mathbf{k}=\left(k_{x}, k_{y}, k_{z}\right)$ is the unitary wave-vector, and $\mathbf{e}_{\mathbf{0}}=\left(a \mathbf{e}_{\mathbf{x}}+b \mathbf{e}_{\mathbf{y}}+\right.$ $c \mathbf{e}_{\mathbf{z}}$ ) is a unit vector in Cartesian coordinates. By combining Eq. (1.9) with Eqs. (1.3), (1.4) and (1.5)-(1.8) Maxwell equations can be rewritten as follows:

$$
\begin{aligned}
\mathbf{k} \cdot \mathbf{E} & =0, \\
\mathbf{k} \cdot \mathbf{B} & =0, \\
n \mathbf{k} \times \mathbf{E} & =\mathbf{B}, \\
n \mathbf{k} \times \mathbf{H} & =-\mathbf{E} .
\end{aligned}
$$

Now we can combine Eqs. (1.3), (1.12) and (1.13) to obtain

$$
\hat{\varepsilon} \mathbf{E}=n^{2}[\mathbf{E}-(\mathbf{k} \cdot \mathbf{E}) \mathbf{k}] .
$$

Eq. (1.14) forms a system of three linear equations that can be solved only if the associated determinant vanishes, i.e.

$$
\operatorname{det}\left|\mathrm{n}^{2} \delta_{\mathrm{ij}}-\mathrm{n}^{2} \mathrm{k}_{\mathrm{i}} \mathrm{k}_{\mathrm{j}}-\varepsilon_{\mathrm{ij}}\right|=\left|\begin{array}{ccc}
n^{2}\left(1-k_{x}^{2}\right)-\varepsilon_{1} & n^{2} k_{x} k_{y} & n^{2} k_{x} k_{z} \\
n^{2} k_{y} k_{x} & n^{2}\left(1-k_{y}^{2}\right)-\varepsilon_{2} & n^{2} k_{y} k_{z} \\
n^{2} k_{z} k_{x} & n^{2} k_{z} k_{y} & n^{2}\left(1-k_{z}^{2}\right)-\varepsilon_{3}
\end{array}\right|=0 .
$$


Evaluation of Eq. (1.15) gives

$$
n^{4}\left(\varepsilon_{1} k_{x}^{2}+\varepsilon_{2} k_{y}^{2}+\varepsilon_{3} k_{z}^{2}\right)-n^{2}\left[k_{x}^{2} \varepsilon_{1}\left(\varepsilon_{2}+\varepsilon_{3}\right)+k_{y}^{2} \varepsilon_{2}\left(\varepsilon_{1}+\varepsilon_{3}\right)+k_{z}^{2} \varepsilon_{3}\left(\varepsilon_{1}+\varepsilon_{2}\right)\right]+k_{x}^{2} \varepsilon_{1} \varepsilon_{2} \varepsilon_{3}=0 \text {. }
$$

Eq. (1.16) which is a surface of the $4^{\text {th }}$ order known as Fresnel's equation and it is probably the most relevant equation of crystal optics, since it describes light propagation in isotropic, uniaxial and biaxial media. Eq. (1.16) has, in general, two different real roots for a fixed $\mathbf{k}$, i.e. every input plane-wave into an anisotropic medium propagates as two plane-waves with different wave-vectors within the medium.

Let us here note that Fresnel's wave-surface describes phase propagation within the material. At variance with isotropic homogeneous media, in anisotropic crystals the direction of propagation of the energy, i.e. the direction of the Poynting vector, does not coincide with the direction of propagation of the phase, in general. An equivalent equation to Eq. (1.16) can be obtained analogously for propagation of rays within the crystal [6,7].

\subsubsection{Uniaxial crystals}

Uniaxial crystals are optical media with only two different permittivity constants $\varepsilon_{1}=\varepsilon_{2} \equiv \varepsilon_{o}$ and $\varepsilon_{3}=\varepsilon_{e}$, being $\varepsilon_{o}$ parallel to the optic axis of the crystal and $\varepsilon_{e}$ orthogonal to it. Under this condition, Eq. (1.16) reads as

$$
\left(n^{2}-\varepsilon_{o}\right)\left[n^{2} \varepsilon_{e} k_{z}^{2}+n^{2} \varepsilon_{e}\left(k_{x}^{2}+k_{y}^{2}\right)-\varepsilon_{o} \varepsilon_{e}\right]=0 .
$$

It is straightforward to realize that Eq. (1.17) has two possible solutions: a spheroid and an ellipsoid

$$
\begin{aligned}
k_{x}^{2}+k_{y}^{2}+k_{z}^{2} & =\frac{\varepsilon_{o}}{n^{2}}, \\
\frac{k_{z}^{2}}{\varepsilon_{o}}+\frac{k_{x}^{2}+k_{y}^{2}}{\varepsilon_{e}} & =\frac{1}{n^{2}} .
\end{aligned}
$$

That is to say, the wave-vector surface of the $4^{\text {th }}$ order becomes two independent surfaces of the $2^{\text {nd }}$ order. Fig. 1.1 gives cuts in the (a) $x y$, (b) $x z$ and (c) $y z$ planes and also (d) the three-dimensional surface of Eq. (1.17) for $\varepsilon_{o}=1$ and $\varepsilon_{e}=2$. Refraction of an input plane-wave by a uniaxial crystal is depicted in Fig. 1.1(e) by the direction of the normals of the tangent planes with red and blue lines at the crossing points between the input ray (black dashed line) with the Fresnel surfaces. One of the refracted planewaves follows usual laws of refraction in a medium with refractive index $n=\sqrt{\varepsilon_{o}}$ and is called as the ordinary wave, depicted in red in Fig. 1.1(e). In contrast, the direction of propagation of the other refracted wave depends on the angle that the input plane-wave makes with the optic axis and is known as the extraordinary wave, depicted in blue in Fig. 1.1(e). As it can be appreciated, the spheroid and the ellipsoid touch each other at two points. The line that connects these two points gives the direction of the optic axis 
of the crystal. Note that the refracted waves from a plane-wave propagating along the optic axis overlap and therefore only a single plane-wave is observed. If the spheroid is contained within the ellipsoid, the material is said to be a positive uniaxial crystal, while if the case is the opposite, the material is a negative uniaxial crystal. Note additionally that at the crossing point between the two surfaces there can be found only one tangent plane that touches the surfaces at a single point.

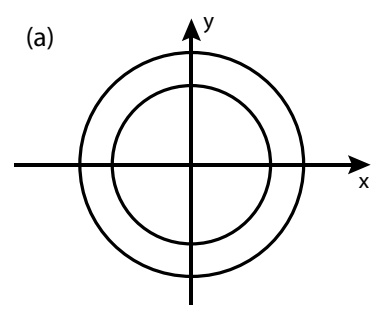

(d)

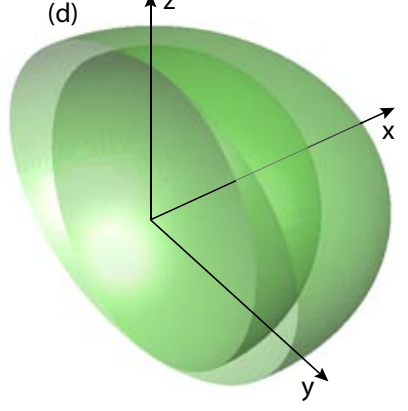

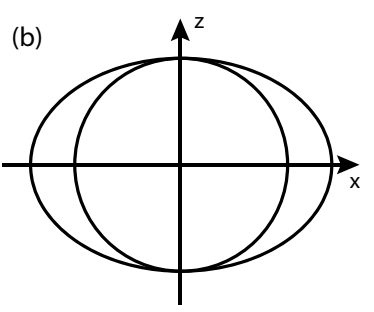

(e)

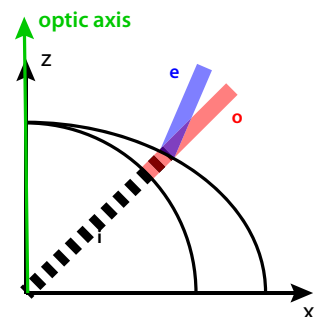

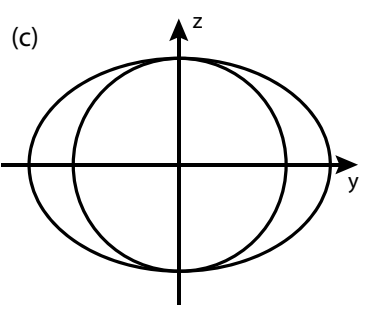

(f)

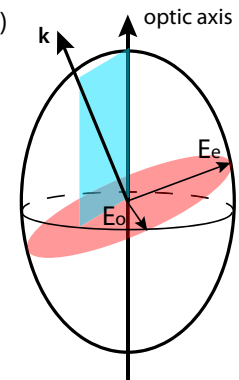

Figure 1.1: Transverse cuts in the (a) $x y$, (b) $x z$ and (c) $y z$ planes of the wave-surface. (d) Three-dimensional representation of the wave-surface of Eq. (1.17) for $\varepsilon_{o}=1$ and $\varepsilon_{e}=2$. (e) An input plane-wave (depicted with a black dashed line) refracts into an ordinary (red) and extraordinary (blue) plane waves, which are in the direction of the normal vectors to the tangent plane at the crossing point between the input wavevector and Fresnel's surface. (f) The ordinary and extraordinary waves are mutually orthogonally polarized.

The state of polarization of the ordinary and extraordinary waves can be easily visualized through the construction depicted in Fig. 1.1(e). From Eq. (1.10) it is clear that $\mathbf{E} \perp \mathbf{k}$. Let's consider the plane containing the wave-vector $\mathbf{k}$ of a propagating plane-wave through the crystal and the optic axis. This plane is known as the principal plane (depicted in color blue). The section of the plane perpendicular to $\mathbf{k}$ at the origin with the surface of wave normals forms an ellipse (depicted in color red) whose principal semi-axes are one perpendicular and one parallel to the principal plane. At the same time, the semi-axis of the ellipse perpendicular to the principal plane has the same radius than the spheroid, i.e. it is in the direction of the ordinary wave. Therefore, the electric field of the ordinary wave vibrates orthogonal to the principal plane, while the electric field of the extraordinary wave vibrates parallel to this plane being both electric fields mutually orthogonal. Note that for a given input plane-wave, the ordinary and 
extraordinary refracted waves are always linearly polarized. The combination of both waves gives a total elliptically polarized wave, in general.

\subsubsection{Biaxial crystals}

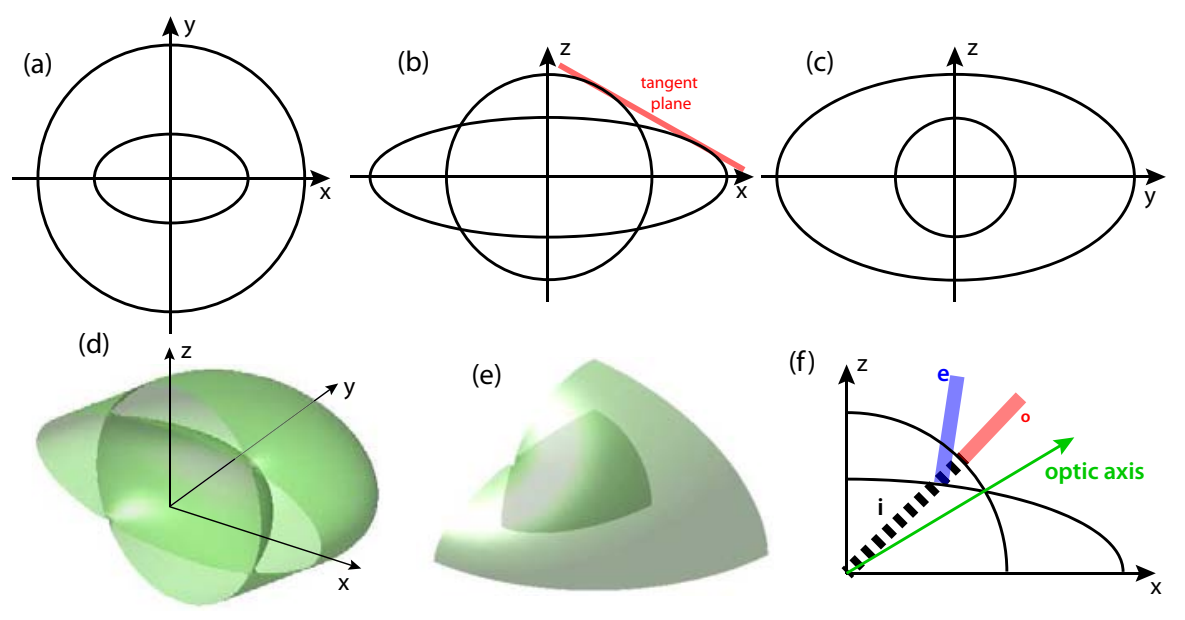

Figure 1.2: Transverse cuts in the (a) $x y$, (b) $x z$ and (c) $y z$ planes of the wave-surface. (d) Three-dimensional representation of the wave-surface surface of Eq. (1.16) for $\varepsilon_{1}=1$, $\varepsilon_{2}=3$ and $\varepsilon_{3}=5$. (e) A single octane of the wave surface to see properly the crossing point between both surfaces. We have used illumination parallel to the optic axis to show that instead of a point, a ring is reflected. (f) An input plane-wave (depicted with a black dashed line) refracts into an ordinary (red) and extraordinary (blue) plane waves, which are in the direction of the normal vectors to the tangent plane at the crossing point between the input wave-vector and Fresnel's surface.

Biaxial crystals are anisotropic optical materials with three different permittivity constants, i. e. $\varepsilon_{1} \neq \varepsilon_{2} \neq \varepsilon_{3}$. In this case, further simplifications of Eq. (1.16) cannot be applied and the wave-surface is of the $4^{\text {th }}$ order. All the phenomenology associated to refraction of an input plane wave when it propagates within a biaxial crystal along any direction and described by Fresnel's surface is analogous to a uniaxial crystal: the input plane-wave refracts as two orthogonally linearly polarized waves that propagate along different directions given by the normals to the tangent plane of the surfaces at the crossing point with the input plane-wave. However, at variance with uniaxial crystals, the two wave surfaces generated in a biaxial crystal cross each other at 4 points. The two lines that connect each pair of diametric crossing points of the surfaces give the direction of the optic axes of the crystal. Fig. 1.2 gives cuts in the (a) $x y$, (b) $x z$ and (c) $y z$ and also (d) the three-dimensional surface of Eq. (1.16) for $\varepsilon_{1}=1, \varepsilon_{e}=2$ and $\varepsilon_{e}=3$. Fig. 1.2(e) shows only an octant of the total volume to facilitate the visualization of the crossing point between the two surfaces. and Fig. 1.2(f) depicts refraction in a biaxial crystal. From Fig. 1.2(b) it is clear that at the crossing points defining the optic axes, 
a tangent plane cannot be defined. Nevertheless there can be found a tangent plane enclosing the optic axis, that it is expected to touch the surface at two points. However, the octane from Fig. 1.2(e) shows that when one shines light in the direction of the optic axis (see white illumination), the reflected light forms neither a spot nor a double-spot but a ring. As a consequence, the tangent plane enclosing the optic axis does not touch the surface at two points but at a ring. In what follows we will show that this apparently small difference between uniaxial and biaxial crystals is more relevant than it seems at first sight.

\subsection{The subtle phenomenon of conical refraction}

As commented in the previous section, there are two types of anisotropic optical media: uniaxial and biaxial crystals. In both types of crystals, an incident light beam suffers, in general, from double refraction at the exit of the crystal. However, there exist a significant difference that escaped to Fresnel between the two types of media when the beam propagates parallel to the optic axis. Fresnel found correctly that the general form of the wave surface is a doubled sheeted surface of the $4^{\text {th }}$ order and that the crossing points between the two sheets give the optic axes of the crystal. According to the experimental observations, he stated that light propagating parallel to the optic axis does not suffer from double refraction. This statement, which is true for uniaxial crystals, does not hold for biaxial crystals or, at least, not completely.

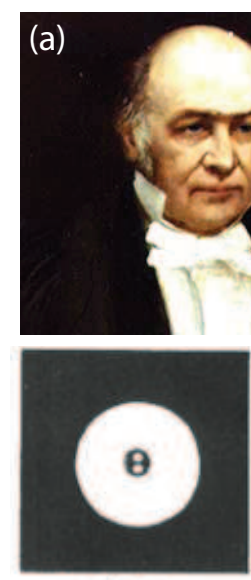

Fig. a.
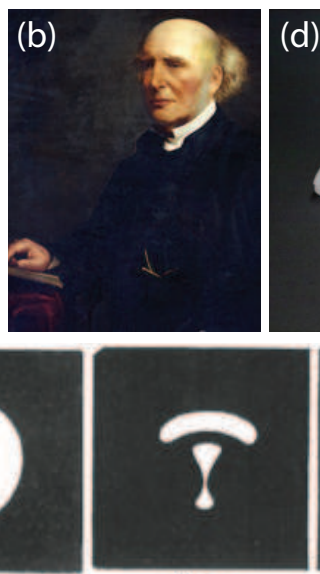

Fig. o.
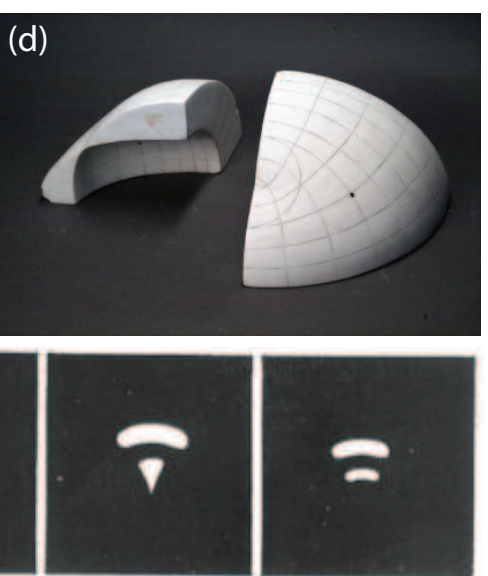

Fig. 4.

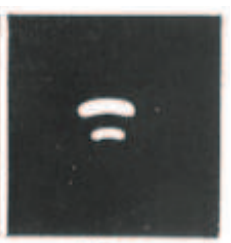

Fig, e.

Figure 1.3: The fathers of CR: (a) W. R. Hamilton and (b) H. Lloyd. (c) Model of the wave surface of a biaxial crystal reproduced by the Göttingen Collection of Mathematical Models and Instruments. Bottom sequence of images: transition from double refraction of CR reproduced from Lloyd report 8 .

William Rowan Hamilton was one the first great mathematicians of the $19^{\text {th }}$ century and one of the fathers of the modern formulation of physics as we recognize it nowadays. 
He was very interested on the properties of the Fresnel wave surface and studied it in detail, discovering new properties that were misapprehended by all previous researchers. It was clear that the direction of the refracted beams in biaxial crystals can be described by the normal vector to the tangent planes to the surface at any point. The subtle realization of Hamilton was that at the crossing points of the Fresnel surface, i.e. at the points defining the optic axes, there is not a unique tangent plane. As shown in Fig. 1.2(a), the crossing points form conoidal cusps where the tangent plane is not well defined. In contrast, at that singular points a tangent cone can be found. Additionally, Fresnel assumed that each of the four singularities could be completely covered over by a tangent plane that touches the wave surface at two single points, as suggested by the two points $a$ and $b$ from Fig. 1.2, However, the complex structure of the wave surface when imaged in three dimensions [Fig. 1.2(b)] shows that a closing tangent plane touches the wave surface along a ring, as Hamilton discovered. Therefore, a bundle of waves of light propagating parallel to one of the optic axes of a biaxial crystal should refract internally as an slanted cone within the crystal and emerge from it as an hollow light cylinder whose transverse cross-section forms a ring of light. But not only this, there is one more solution in which a bundle of waves forming a cone of light propagate as a pencil within the crystal and emerge externally as a cone of light. These two new phenomena were called as internal and external conical refraction (CR) respectively by Hamilton. Hamilton's theory of CR was presented together with a general method to solve optical problems in his Essay on the theory of systems of rays in 1832 [9].

The two new phenomena predicted elegantly by Hamilton needed to be observed experimentally. For that reason, Hamilton contacted Humphrey Lloyd and asked him to carry out the corresponding experimental observations. He took aragonite as biaxial material and succeeded in observing, first, external and, secondly, internal CR few weeks after Hamilton's prediction [8] from sunlight and by arranging a metallic pinhole at the entrance of the crystal. In addition to the novel refraction phenomena, Lloyd observed that all the rays of the light cone were polarized in different planes so that every two diametrically opposite points have orthogonal polarizations. This new law of polarization in CR was deduced afterwards by Hamilton from his theory. Lloyd also observed that the light cylinder appeared displaced with respect to the transverse position of the pinhole.

Hamilton's discovery together with Lloyd's observations was the definitive fist on the table of the wave theory of light and it meant its acceptance over the corpuscular theory. It had the relevance of being one of the first mathematical predictions of physical phenomena, at variance with previous theoretical contributions to the wave theory of light that were explanations of already observed effects. However, this was not the last word in CR since, as we show in the next section, new phenomena escaping Hamilton's theory were observed later. 


\subsection{Everything was not said: new observations on conical refraction}

It seemed that Hamilton's theory was able to predict any phenomena regarding light propagation in optical media and, in particular, that all the phenomenology associated to CR had been reported. However, in 1839 Johann Christian Poggendorff (editor of Annalen der Physik) reported new observations on light propagation under internal CR conditions [10]. As Lloyd, he used aragonite as biaxial material but as a light source he used a fire lamp, which tends to be more monochromatic than sunlight, and a lens. In addition to the transition from double refraction to conical refraction as the optic axes of the crystal and the direction of the beam approach each other and the polarization distribution along the ring, Poggendorff realized that the ring of light was split by a ring of complete darkness. Two years after, similar observations were reported by $\mathrm{R}$. Potter [11, although he resisted to accept that his experimental results confirmed the wave nature of light.

The work of Poggendorff caused some impression in their German colleagues Beer and Haidinger, who reported independently very detailed experimental works on the phenomenology of internal CR: the transition from double to internal conical refraction, the state of polarization of the ring, the dispersion relation for different wavelengths and even some calculations on the refractive indices of aragonite but they did not say anything regarding the dark ring observed by Poggendorff [12,13].

During the following years, it seems that interest on studying CR and on explaining the novel observations from Poggendorff disappeared. In 1905, Voigt presented an exhaustive 50 pages long article with both an experimental and a theoretical analysis of CR in Annalen der Physik, where he discussed all the phenomenology known and gave a theoretical explanation for the Poggendorff dark ring [14. He gave a guide of all types of crystallographic groups and included also crystals with optical activity. Voigt's proposal for the Poggendorff dark ring was not too far from reality: the biaxial crystal generates a conical wave for the ordinary and the extraordinary polarizations and each of the two bright rings split by the Poggendorff dark ring correspond to one of that ordinary and extraordinary conical waves. In addition, Voigt reported that the lateral shift of the light cylinder observed by Lloyd depends on the orientation of the plane of optic axes of the crystal. Finally, he also realized that if the incident beam is linearly polarized, the ring lacks of one sector.

Hamilton was not the only big presence in CR. In 1941 the Nobel prize winner Sir Chandrasekhara Venkata Raman published a series of works on internal CR in naphthalene crystals [15 17, a biaxial material with almost 10 times larger birefringence than aragonite. He confirmed the presence of the Poggendorff dark ring and reported a new observation: the transverse intensity pattern along the beam propagation direction changes. There is one plane at which the Poggendorff ring can be observed clearly and as the imaging plane is moved from that plane the bright rings become wider. Far 
enough from the plane of observation of the Poggendorff ring, the inner ring becomes a bright axial spot. These observations had to wait for a diffractive theory of CR to have a theoretical explanation.

\subsection{From Hamilton's model to the diffractive theory of conical refraction}

The born of non-linear optics brought a new hope for the study of CR mainly by two different reasons: (1) the requirement of good quality non-linear crystals, being many of them biaxial, and (2) the use of a coherent, monochromatic and easily addressable light source, i. e. the laser. In this sense, we find the first proposal of a new generation of experiments in CR in the work of Goyal and Prakash [18], where they discuss the possibility of observing $\mathrm{CR}$ in a non-linear medium whose refractive index is modified by means of an additional high intense light beam. Almost at the same time, Portigal and Burstein made the first big step towards a complete description of the CR phenomenon [19]. Starting from Maxwell equations, they were able to give a quantitative analysis of internal CR by calculating the phase velocities, polarization modes and Poynting vector of a bundle of waves entering into the biaxial crystal.

Soon afterwards, Lalor presented a series of works in which he calculated The Angular Spectrum of Electromagnetic Fields in Crystals both for uniaxial and biaxial crystals [20, 21]. This was, up to our knowledge, the first attempt to describe light propagation in anisotropic media with a diffractive formalism. The summit of his series of three papers was An Analytical Approach to the Theory of Internal Conical Refraction, where the Fourier transform has a central role in the description of the phenomenon [22]. Closely related to this work is the one from Schell and Bloembergen 23. In addition to the diffractive equations for the light field out of the biaxial crystal, they also showed for the first time experimental images of CR for linearly polarized input beams with a clear dark ring splitting the ring of CR into two bright rings. Similar observations were reported by Perkal'skis and Mikhailichenko [24] and by Velichkina et al. [25].

In 1978 Belsky and Khapalyuk presented the holy grail of CR: the full diffractive theory of both external [26] and internal CR 27] in biaxial crystals. This work is very related to Lalor's calculations but the equations at which Belsky and Khapalyuk arrived were much more compact. In this formalism, the electric field behind the crystal is obtained from the transverse plane-wave decomposition of the input beam transformed by a Fourier-like operation that includes beam propagation. These results describe in detail all the phenomenology associated with CR, which were demonstrated by Fève et al. with spheres of KTP [28]. The Beslky and Khapalyuk theory of CR was explored in detail by Belsky and Stepanov [29] (including gyroscopic crystals [30]), who recognized the relevance on the $\mathrm{CR}$ beam evolution of the ratio between the ring radius and the waist radius of the input beam, and also by Belafhal for the asymptotic case of $R_{0} \gg w_{0}$ [31], 
where $R_{0}$ is the geometric approximation of the $\mathrm{CR}$ ring radius and $w_{0}$ is the waist radius of the input beam.

172 years after Hamilton's prediction of CR, Sir Michael Victor Berry developed the definitive theory of CR [32]. He considered diffractive paraxial optics and the biaxial crystal acting as a unitary transformation over the angular plane wave spectrum of the input beam impinging the crystal. He presented analytical formulas for the Poggendorff dark and bright rings and for the Raman axial spot. This approach was a breakthrough in biaxial crystal optics and, together with some illustrative experiments on $\mathrm{CR}$ with high quality $\mathrm{KGd}\left(\mathrm{WO}_{4}\right)_{2}$ biaxial crystals [33, and opened a new era for the study of the phenomenon and the applications of CR.

Finally, it is worth saying external $\mathrm{CR}$ has not been reported clearly. The only related experiments are the first one reported by Lloyd [8] and the work from Féve et al. 28]. In this thesis we will always deal with internal CR.

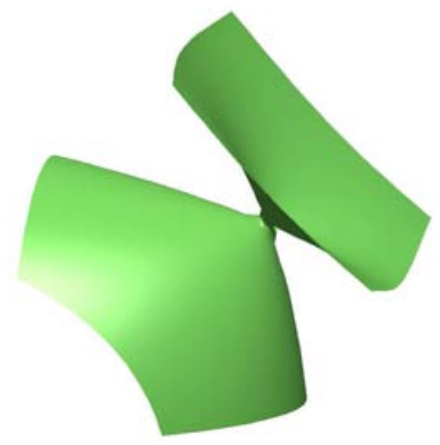

Figure 1.4: Diabolo-like geometry near the cusp point of the Fresnel's surface of a biaxial crystal defining one of the optic axis.

\subsection{Conical refraction out of crystal optics}

CR has been also studied out of the domain of optics. As in many other topics, $\mathrm{CR}$ has its analogous in sound waves by considering sonic crystals 34. Most related works are purely theoretical [35-40] and only preliminary experiments have been reported 4143. However, all the phenomenology related to $\mathrm{CR}$ has been recovered for the acoustic case; namely the generation of conical structures when the incident bundle of waves propagate nearly parallel to the optic axis of the sonic crystal such that the state of polarization along the ring is linear, with the azimuth varying as $\mathbf{e}_{\mathrm{CR}}=(\cos (\phi / 2), \sin (\phi / 2))$. A similar approach has been discussed in plasma physics by Tsiklauri, who reported theoretically CR of magnetohydrodynamical waves in a collisionless plasma with anisotropic thermal pressure [44]. As it has been discussed in previous sections, CR is associated to a diabolo-like cusp in the Fresnel's surface of a biaxial crystal, see Fig. 1.4. These type of surface singularities are conical intersections, which have studied en chemistry for instance [45]. In the line of conical intersections, CR 
has been discussed recently in honeycomb lattices [46,47] and in Lieb lattices [48]. In particular, CR and gap solitons in photonic honeycomb lattices have been reported both experimentally and theoretically by Peleg et al. [49]. Additonally, Bulgakov and Fedorin discussed CR in periodic semiconductors under the influence of an external magnetic field [50]. Finally, note that both these works and our work only consider non-magnetic biaxial crystals. However, it could be interesting to study all the phenomenology associated to the the case of biaxial crystals with $\mu \neq \mu_{0}$, as it has been introduced by Matos and co-workers [51]. 

CHAPTER 2

Theory and fundamental experiments on conical refraction

In this Chapter we introduce theory of CR that will be the base of all the following Chapters and which can be divided in three main contributions. Firstly, we briefly introduce the development of the CR theory, see Section 2.1. In Section 2.2 we present the diffractive theory of CR developed by Belsky, Khapalyuk and Berry (BKB) and show the cylindrically symmetric solution that is the base of almost all the CR-related works. Our contribution to this Section is the demonstration that the BKB solution can be used to predict the transformation of beams with non-cylindrically symmetric electric field amplitude propagating both along and out of the optic axis of a biaxial crystal. Then, Section 2.3 we propose a simple formulation based on splitting of linearly polarized wave-vectors that can be used to predict the CR transverse intensity pattern of arbitrary input beams at the focal plane, and demonstrate it for an axicon beam. In Section 2.4 we reformulate the dual-cone model of $\mathrm{CR}$, give an intuitive explanation based on the wave-vector and polarization dependence of the CR phenomenon and demonstrate the former experimentally. The wave-vector and polarization CR formalism is extended to a cascade of multiple biaxial crystals with aligned optic axis, including linearly polarized input beams, in Section 2.5. We show that, in the general case, a cascade of $N$ crystals generates $2^{N-1}$ concentric rings at the focal plane. Finally, in Section 2.6, we discuss the main conclusions of the Chapter.

The research contained in this chapter has been published in Refs. [52 56] and has been done in collaboration of Alba Peinado, Ángel Lizana, Irene Estévez and Juan Campos from the Universitat Autònoma de Barcelona; Hiromitsu Tomizawa from the Japan Synchrotron Radiation Research Institute, Asticio Vargas and Fabián A. TorresRuiz from the Universidad de Concepción; and Ignacio Moreno from the Universidad Miguel Hernández. 


\subsection{Introduction}

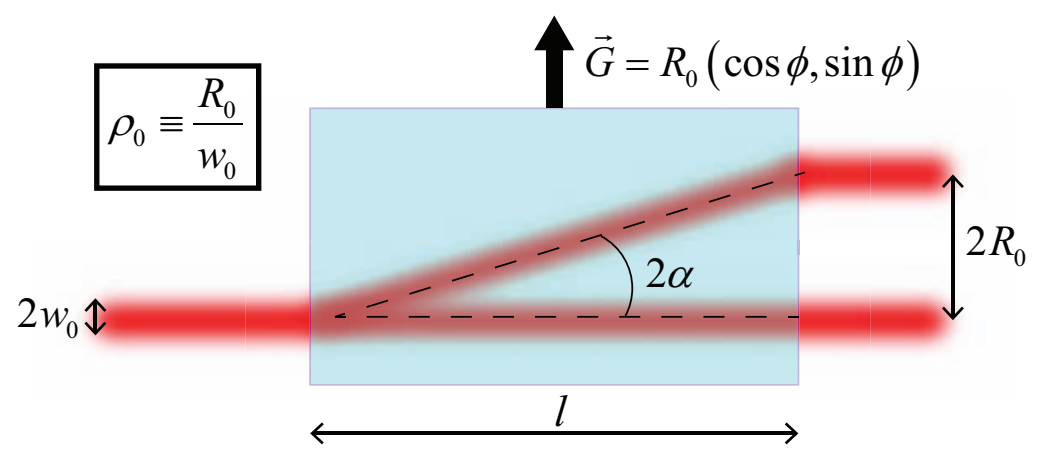

Figure 2.1: Basic parameters in CR. $R_{0}$ is the geometric approximation of the CR light ring that can be calculated as $R_{0}=l \alpha$, where $l$ is the length of the biaxial crystal and $\alpha$ the semi-angle of the light cone or conicity. $\rho_{0}$ measures the ring radius in units of waist radius of the input beam $w_{0} . \mathbf{G}$ is a vector that can be attached to the crystal to describe both the direction and the magnitude of the lateral displacement that the CR ring suffers. $\phi$ also gives the orientation of the plane of optic axes of the crystal.

As stated in the previous Chapter, CR was predicted theoretically by Hamilton by analyzing in detail Fresnel's surface corresponding to a biaxial crystal. He considered a collimated bundle of plane-waves propagating within the crystal and parallel to one of the optic axes and deduced that the bundle would refract as an slanted cone within the crystal and emerge as a hollow light cylinder whose transverse profile forms a light ring. Although this is precisely what was found by Lloyd, further experiments carried out by Poggendorff, Voigt and Raman showed that Hamilton theory could not describe completely all the phenomenology associated to CR. These results surpassing Hamilton's theory come from the fact that a collimated beam is an idealization without real equivalent. In any experimental situation, beams have always a plane at which their width is minimum. In other words, real beams are focused in a greater or lesser extent. The description of the transformation of focused beams by optical systems was far from Hamilton's age and was not well established until the middle of the $20^{\text {th }}$ century. That is the reason why the diffractive theory of CR, capable of predicting all the observed phenomena, had to wait until the last quarter of the last century. This theory was presented by Belsky and Khapalyuk [26,27] and then reformulated in an elegant way by Berry [32]. Recently, we have shown that the reformulation of the diffractive theory of CR carried out by Berry can be used to predict the beam evolution of light propagation in biaxial crystals along any direction and not only along the optic axis [52] and also in case of non-homogeneously polarized beams [53]. Note that alternatively Dreger [57] and Garnier [58] have reported the solutions of optical beam propagation in biaxial crystals, although in both cases the theory presented is very involved. On the other hand, Sokolovskii and co-workers showed that the Belsky-Khapalyuk-Berry equations 
can be re-arranged so that they describe CR in terms of the interference between two co-propagating cones [59], being this theory demonstrated experimentally and newly re-formulated by us [56], as it is shown in Section 2.4. Alternatively, we have also presented a novel proposal that can be used to easily predict the CR pattern at the focal plane by means of a wave-vector and polarization formalism [54 that can be also used in a cascade of biaxial crystals, as we have also shown in Ref. [55]. In this chapter, we present these three theories and study in detail all the characteristics associated to CR both for single and multiple crystals.
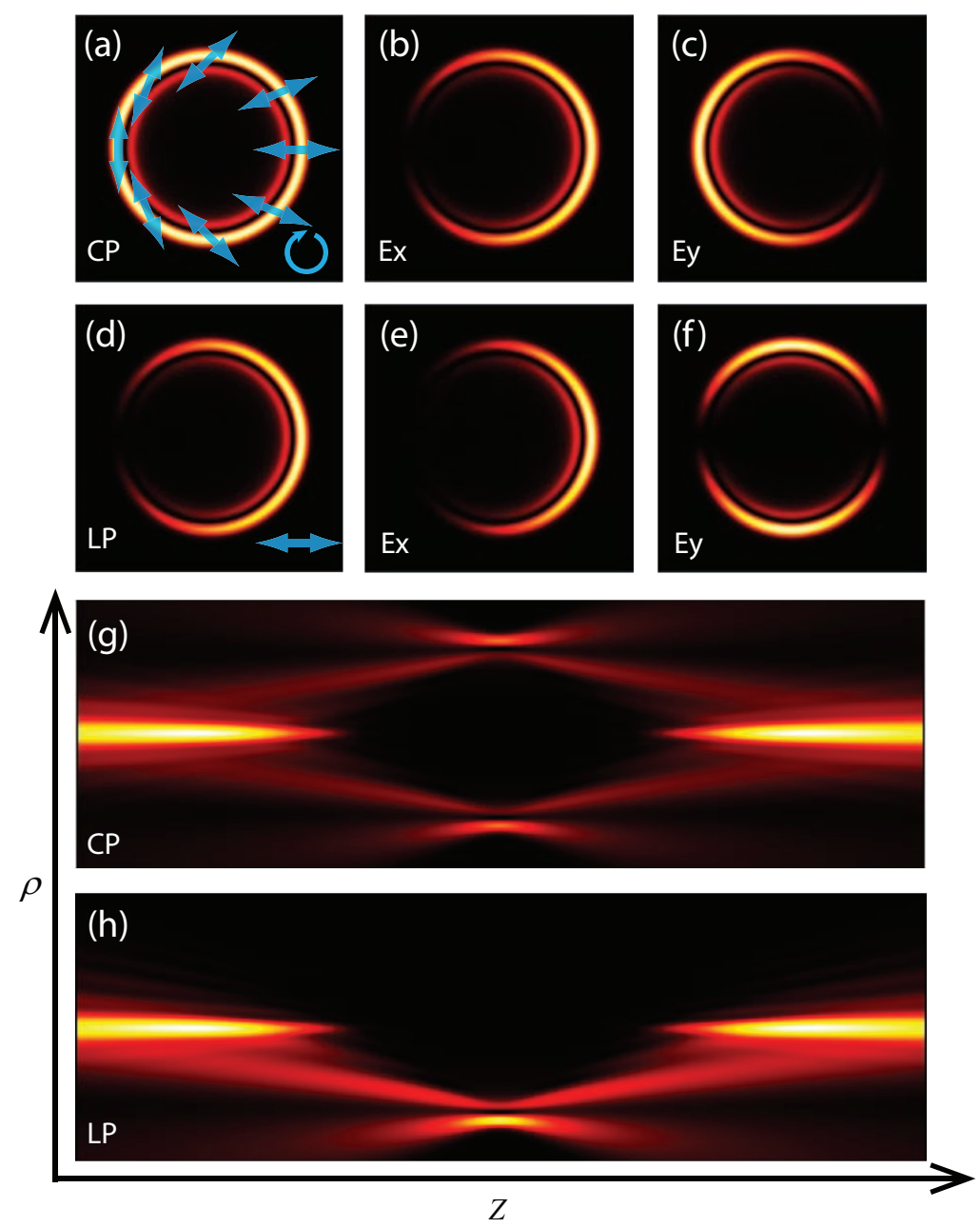

Figure 2.2: Conical refraction for a Gaussian input beam with left handed circular polarization [images (a)-(c) and (g)] and linear horizontal polarization [images (d)-(f) and $(\mathrm{h})]$. Images (a) and $(\mathrm{d})$ are transverse cuts at the focal plane $(Z=0)$ of the intensity pattern. Images (b) and (e) are the transverse electric field in the $X$ direction, while images (b) and (e) are the transverse electric field in the $Y$ direction. (g) and (h) are transverse cuts in the $Z-\rho$ plane at $\varphi=0$. 
Let us firstly briefly outline geometrically the CR annular pattern observed at the focal plane with an input Gaussian beam. While the complete CR ring is observed for circular polarization of the input Gaussian beam, as it is shown in Fig. 2.2. (a), a crescent ring with one point of the ring being of zero intensity appears for linear polarization, see Fig. 2.2(d). In both cases, the center of the CR ring is laterally shifted with respect to the incident beam, as sketched in Fig. 2.1. This shift can be represented by a vector $\mathbf{G}=R_{0}\left(\cos \phi_{G}, \sin \phi_{G}\right)$ that belongs to the plane of the crystal optic axes 33. Its modulus is equal to the ring radius $|\mathbf{G}| \equiv R_{0}$. The latter is the product of the crystal length, $l$, and the $\mathrm{CR}$ semi-angle $\alpha$ or conicity, i.e., $R_{0}=l \alpha$ [33]. The CR semi-angle $\alpha$ depends on the principal refractive indices of the crystal as $\alpha=\sqrt{\left(n_{2}^{2}-n_{1}^{2}\right)\left(n_{3}^{2}-n_{2}^{2}\right) / n_{2}^{2}}$, where we have assumed $n_{1}<n_{2}<n_{3}$. For the $\left.\operatorname{KGd}\left(\mathrm{WO}_{4}\right)_{2}\right)$ biaxial crystals that we will use in all our experiments, $\alpha(\lambda=633 \mathrm{~nm})=16.9 \mathrm{mrad}[33,60$.

\subsection{Diffractive solution}

\subsubsection{Cylindrically symmetric solution}

We will present the theory as re-formulated by Berry, since, in our opinion, it is very compact and clear in terms of operators. In what follows, we will consider normalized coordinates to the waist radius $w_{0}$ and and Rayleigh length $z_{R}$ of the input beam, i.e., $X \leftrightarrow x / w_{0}, Y \leftrightarrow y / w_{0}$ and $Z \leftrightarrow z / z_{R}$. In the parabolic approximation, after passing through a medium or optical element, a light beam can be described by means of its displacement vector $\mathbf{D}$ as a superposition of plane waves $\mathbf{k}=\left(k_{x}, k_{y}\right)$, which are generated from a unitary transformation provided by the optical element $\hat{U}(\mathbf{k})$ applied over the Fourier transform vector of the input light beam $\mathbf{A}(\mathbf{k})$. In other words,

$$
\mathbf{D}=\frac{1}{(2 \pi)^{2}} \int_{-\infty}^{\infty} \int^{i \mathbf{k} \cdot \mathbf{r}} \hat{U}(\mathbf{k}) \mathbf{A}(\mathbf{k}) d \mathbf{k}
$$

where $\mathbf{A}(\mathbf{k})=\left(A_{x}(\mathbf{k}),+A_{y}(\mathbf{k})\right.$ is the $2 \mathrm{D}$ Fourier transform of the transverse amplitude of the input electric field $\mathbf{E}(\mathbf{r})=\left(E_{x}(\mathbf{r})+E_{y}(\mathbf{r})\right)$ :

$$
\begin{aligned}
& A_{x}(\mathbf{k})=\frac{1}{(2 \pi)^{2}} \int_{-\infty}^{\infty} \int_{x}(\mathbf{r}) e^{-i \mathbf{k} \cdot \mathbf{r}} d \mathbf{r}, \\
& A_{y}(\mathbf{k})=\frac{1}{(2 \pi)^{2}} \int_{-\infty}^{\infty} \int_{y}(\mathbf{r}) e^{-i \mathbf{k} \cdot \mathbf{r}} d \mathbf{r} .
\end{aligned}
$$

For a low birefringent biaxial crystal, Berry showed that the unitary transformation provided by the medium is $\hat{U}(\vec{\kappa})=e^{-i \vec{\Gamma}(\vec{\kappa})}$ with 32]

$$
\boldsymbol{\Gamma}(\vec{k})=\frac{1}{2} k^{2} Z^{2} \hat{\mathbf{I}}+\rho_{0} \mathbf{k} \cdot\left(\hat{\sigma_{3}}, \hat{\sigma_{1}}\right),
$$


where $\hat{\sigma}_{3}$ and $\hat{\sigma}_{1}$ are the Pauli matrices and $\hat{\mathbf{I}}$ is the $2 \times 2$ identity matrix. $\rho_{0}$ is the ratio between the geometric ring radius of the $\mathrm{CR}$ ring $R_{0}$ and the waist radius of the focused input beam $w_{0}$, i.e., $\rho_{0} \equiv R_{0} / w_{0}$. We would like to note that this transformation is assumed in the formulation of Belsky and Khapalyuk [26, 27]. It is straightforward to demonstrate that for a given vector $\mathbf{v}=v \mathbf{n}$ with $|n|=1$, the evaluation of $e^{i v(\vec{n} \cdot \vec{\sigma})}$ gives $\hat{\mathbf{I}} \cos (v)+i(\mathbf{n} \cdot \sigma \sin (v))$, where $\sigma=\left(\hat{\sigma}_{1}, \hat{\sigma}_{2}, \hat{\sigma_{3}}\right)$ is the vector of Pauli matrices. By recalling the latter identity and after evaluation of $\hat{U}(\mathbf{k})=e^{-i \Gamma(\mathbf{k})}$ by using Eq. (2.4), the unitary transformation provided by the crystal can be obtained:

$$
\hat{U}(\mathbf{k})=e^{-i \frac{1}{2 n} k^{2} Z^{2}}\left[\cos \left(\rho_{0} k\right) \hat{\mathbf{I}}+i \frac{\sin \left(\rho_{0} k\right)}{k} \mathbf{k} \cdot\left(\hat{\sigma_{3}}, \hat{\sigma}_{1}\right)\right]
$$

where $k \equiv \sqrt{k_{x}^{2}+k_{y}^{2}}$ is the transverse magnitude of the wave-vector in cylindrical coordinates and $n$ is the mean refractive index of the crystal. By using Eq. (2.5), Eq. (2.1) becomes

$$
\mathbf{D}=\frac{1}{(2 \pi)^{2}} \int_{-\infty}^{\infty} \int_{\infty}^{i \mathbf{k} \cdot \mathbf{r}} e^{-i \frac{1}{2 n} k^{2} Z^{2}}\left[\cos \left(\rho_{0} k\right) \hat{\mathbf{I}}+i \frac{\sin \left(\rho_{0} k\right)}{k} \mathbf{k} \cdot\left(\hat{\sigma}_{3}, \hat{\sigma}_{1}\right)\right] \mathbf{A}(\mathbf{k}) d \mathbf{k}
$$

which can be written in cylindrical coordinates $\left(\mathbf{k}=k\left(\cos \left(\phi_{k}\right), \sin \left(\phi_{k}\right)\right), \quad \mathbf{r}=\right.$ $\left.r(\cos (\varphi), \sin (\varphi)) / w_{0}=\rho(\cos (\varphi), \sin (\varphi))\right)$ as

$$
\mathbf{D}=\frac{1}{(2 \pi)^{2}} \iint e^{i k \rho \cos \left(\phi_{k}-\varphi\right)} e^{-i \frac{1}{2 n} k^{2} Z^{2}}\left(\begin{array}{cc}
\cos \left(\phi_{k}\right) & \sin \left(\phi_{k}\right) \\
\sin \left(\phi_{k}\right) & -\cos \left(\phi_{k}\right)
\end{array}\right) \mathbf{A}(\mathbf{k}) k d \mathbf{k} .
$$

Now, we simplify the system by considering a uniformly polarized and cylindrically symmetric input beam $\mathbf{E}=E(r) \mathbf{e}_{\mathbf{0}}$ with cylindrically symmetric Fourier transform too, i.e., $A_{x}(\mathbf{k})=A_{y}(\mathbf{k})=a(k)$, where

$$
a(k)=\int_{0}^{\infty} k E(r, Z=0) J_{0}(r k) d r,
$$

where $J_{n}(x)$ is the $\mathrm{n}^{\text {th }}$-order Bessel function of the first type. With this assumption, we can integrate over $\phi_{k}$ by using the following expressions

$$
\begin{aligned}
\int_{0}^{2 \pi} e^{i k \rho \cos \left(\phi_{k}-\varphi\right)} d k & =J_{0}(k \rho) \\
\int_{0}^{2 \pi} e^{i k \rho \cos \left(\phi_{k}-\varphi\right)} \cos \left(\phi_{k}\right) d k & =\cos (\varphi) J_{1}(k \rho)
\end{aligned}
$$

and obtain a $1 \mathrm{D}$ integral for the field $\mathbf{D}$ :

$$
\mathbf{D}=\left(\begin{array}{cc}
B_{0}+B_{1} \cos \varphi & B_{1} \sin \varphi \\
B_{1} \sin \varphi & B_{0}-B_{1} \cos \varphi
\end{array}\right) \mathbf{e}_{\mathbf{0}}
$$


where $B_{0}=B_{0}\left(\rho, Z, \rho_{0}\right)$ and $B_{1}=B_{1}\left(\rho, Z, \rho_{0}\right)$ are the Belsky-Khapalyuk-Berry integrals that describe the evolution of the $\mathrm{CR}$ beam in both the radial and axial directions:

$$
\begin{aligned}
& B_{0}\left(r, Z, \rho_{0}\right)=\frac{1}{(2 \pi)} \int_{0}^{\infty} a(k) e^{-i \frac{1}{2 n} k^{2} z^{2}} \cos \left(k \rho_{0}\right) J_{0}(k r) d \phi_{k}, \\
& B_{0}\left(r, Z, \rho_{0}\right)=\frac{1}{(2 \pi)} \int_{0}^{\infty} a(k) e^{-i \frac{1}{2 n} k^{2} Z^{2}} \sin \left(k \rho_{0}\right) J_{1}(k r) d \phi_{k} .
\end{aligned}
$$

For an input beam of circular polarization (CP) (also for random polarization, RP) and of linear polarization (LP), the intensity distribution behind the crystal becomes, respectively:

$$
\begin{aligned}
I_{\mathrm{CP}}(\rho, Z) & =\left|B_{C}\right|^{2}+\left|B_{S}\right|^{2} \\
I_{\mathrm{LP}}(\rho, Z) & =I_{\mathrm{CP}}+2 \operatorname{Re}\left[B_{C} B_{S}^{*}\right] \cos \left(2 \Phi-\left(\varphi+\varphi_{C}\right)\right),
\end{aligned}
$$

where $\Phi$ is the polarization azimuth of the LP input beam with $\mathbf{e}^{\mathrm{in}}=(\cos \Phi, \sin \Phi)$.

To explore the main features of the CR beam, let's consider the case of a Gaussian input beam, with electric field and Fourier transform

$$
\begin{aligned}
& \mathbf{E}(\mathbf{r})=E_{0} e^{-\frac{r^{2}}{w(z)^{2}}} \mathbf{e}_{\mathbf{0}} \equiv E_{0} e^{-\rho^{2}} \mathbf{e}_{\mathbf{0}}, \\
& a(k)=a_{0} e^{-\frac{k^{2}}{4}}
\end{aligned}
$$

where $w(z)=w_{0} \sqrt{1+Z^{2}}$ and $E_{0}$ and $a_{0}$ are constants. Additionally, we consider that the dimensions of the $\mathrm{CR}$ ring are much larger than the waist radius of the input beam, i.e., $\rho_{0} \gg 1$. For a circularly polarized beam with $\mathbf{e}_{\mathbf{0}}=1 / \sqrt{2}(1, i)$, at the focal plane $(Z=0)$ the transverse intensity pattern is formed by two bright rings with the characteristic Poggendorff dark annular splitting, see Fig. 2.2(a). Fig. 2.2(b) and (c) show the absolute value of the transverse electric field in the horizontal and vertical directions at the focal plane. As it can be observed, both transverse intensity patterns have a nodal point at diametrically opposite azimuthal positions, which indicates the characteristic polarization distribution of the $\mathrm{CR}$ beam, i.e., the angular variation of the azimuth of the linear polarization along the ring is $\pi$ rad for a full turn. As a consequence, if the input beam is linearly polarized, e.g., for $\mathbf{e}_{\mathbf{0}}=(1,0)$, the transverse intensity pattern forms an azimuthally crescent ring with a point of null intensity coinciding with the point of the ring with orthogonal polarization to the input beam, see Fig. 2.2(d). Fig. 2.2(e) shows a density plot in the $Z-\rho$ plane. Out of the focal plane, along the axial direction, the $\mathrm{CR}$ rings become wider and the Poggendorff splitting disappears as the on-axis intensity (Raman spot) grows up from zero to two axial maxima placed at

$$
Z= \pm \sqrt{4 / 3} \rho_{0} .
$$


Although being the case of a Gaussian input beam the most studied situation in CR for cylindrically symmetric beams [33,61, 69, other works have investigated CR for Laguerre-Gauss beams [70,71] and for top-hat beams [72].

\subsubsection{Non-cylindrically symmetric solution}

Recently, we have demonstrated that the Belsky-Khapalyuk-Berry solution can be also implemented in non-cylindrically symmetric beams [52. Since Eq. (2.5) gives information about the operation of the crystal over an input beam, Eq. (2.11) can always be applied, independently on the transverse profile of the input beam. Additionally, if one realizes that the propagation direction of a beam with transverse amplitude $E(\mathbf{r})$ can be described by the product of this amplitude and a factor $\exp \left(-i \delta \cdot \mathbf{r}_{\perp}\right.$ ) (where $\delta=\left(\delta_{x}, \delta_{y}\right)$ and $\left.\mathbf{r}_{\perp}=(x, y)\right)$, it is straightforward to state that Eq. (2.11) can be implemented with arbitrary beams both in transverse shape and propagation direction. From Eq. (2.11), there can be obtained two main integrals similar to Eq. (2.12) and Eq. (2.13) that describe the beam evolution behind the biaxial crystal [52]:

$$
\begin{aligned}
B_{0, j}\left(\mathbf{r}, \rho_{0}\right) & =\frac{i}{(2 \pi)^{2}} \int_{-\infty}^{\infty} e^{-i\left(\mathbf{k} \cdot \mathbf{r}-\frac{Z}{2 n} k^{2}\right)} \frac{k_{y}}{k} \sin \left(\rho_{0} k\right) A_{j}(\mathbf{k}) d \mathbf{k} \\
B_{1, j}\left(\mathbf{r}, \rho_{0}\right) & =\frac{1}{(2 \pi)^{2}} \int_{-\infty}^{\infty} e^{-i\left(\mathbf{k} \cdot \mathbf{r}-\frac{Z}{2 n} k^{2}\right)}\left(\cos \left(\rho_{0} k\right)+i \frac{k_{x}}{k} \sin \left(\rho_{0} k\right)\right) A_{j}(\mathbf{k}) d \mathbf{k},
\end{aligned}
$$

being $j=(x, y)$. The expressions for the $\mathbf{D}$ field in terms of Eqs. (2.19) and (2.20) are

$$
\begin{aligned}
& D_{x}=B_{0, y}\left(\mathbf{r}, \rho_{0}\right)+B_{1, x}\left(\mathbf{r}, \rho_{0}\right), \\
& D_{y}=B_{0, x}\left(\mathbf{r}, \rho_{0}\right)+B_{1, y}\left(\mathbf{r},-\rho_{0}\right) .
\end{aligned}
$$

Eqs. (2.19)-(2.22) can be used to predict the transformation of any input beam, no matter its state of polarization or its shape, as long as its Fourier transform can be obtained. For input beams with non-homogeneous polarizations, it must be taken into account that the beam and the polarization can always be decomposed in the $(x, y)$ basis, so that the theoretical formalism presented above is always suitable for predicting light propagation in biaxial crystals.

In what follows we discuss the transformation of input beams that propagate within a biaxial crystal non-parallel to one of the optic axes, i.e., under conditions of double refraction. We consider homogeneously left handed circularly polarized input beams, i.e., with normalized electric field $\mathbf{e}_{\mathbf{0}}=(1, i) / \sqrt{2}$, and we look at the transverse patterns at the focal plane $(Z=0)$. We will discuss the already shown case of an input beam with Gaussian transverse profile and also of an elliptical input beam. Their electric field amplitudes are described by

$$
\begin{aligned}
\mathbf{E}_{\mathrm{G}}(x, y) & =e^{-i\left(\delta_{x} x+\delta_{y} y\right)} e^{-\left(x^{2}+y^{2}\right)} \mathbf{e}_{\mathbf{0}} \\
\mathbf{E}_{\mathrm{EB}}(x, y) & =e^{-i\left(\delta_{x} x+\delta_{y} y\right)} e^{-\left(\frac{x^{2}}{a^{2}}+\frac{y^{2}}{b^{2}}\right)} \mathbf{e}_{\mathbf{0}},
\end{aligned}
$$


where $a$ and $b$ are constants and $\delta_{x}$ and $\delta_{y}$ give the angular separation of the input beam's propagation direction with respect to the optic axis of the crystal.

Fig. 2.3 shows transition from double refraction to CR for the Gaussian input beam (first and second rows) and the elliptical input beam (third and fourth rows) both experimentally (second and fourth rows) and numerically calculated by using Eqs. (2.19)(2.24) (first and third rows). For simplicity, we consider only angular displacement of the input beam in the vertical direction, i.e., $\delta_{x}=0$. The experiments have been performed by employing a $\mathrm{KGd}\left(\mathrm{WO}_{2}\right)_{4}$ biaxial crystal with a length of $l=28 \mathrm{~mm}$, yielding a CR ring radius of $R_{0}=l \alpha=475$ mum. The Gaussian input beam used was focused by a spherical lens with $100 \mathrm{~mm}$ of focal length. The elliptical input beam was obtained by focusing the same Gaussian beam with a cylindrical lens with $100 \mathrm{~mm}$ of focal length. The biaxial crystal was mounted on an angular micrometric positioner that allowed to change the $\phi$ and $\theta$ angles in spherical coordinates and to observe the transition from double refraction to $\mathrm{CR}$ as the optic axis and the beam propagation direction approached each other.

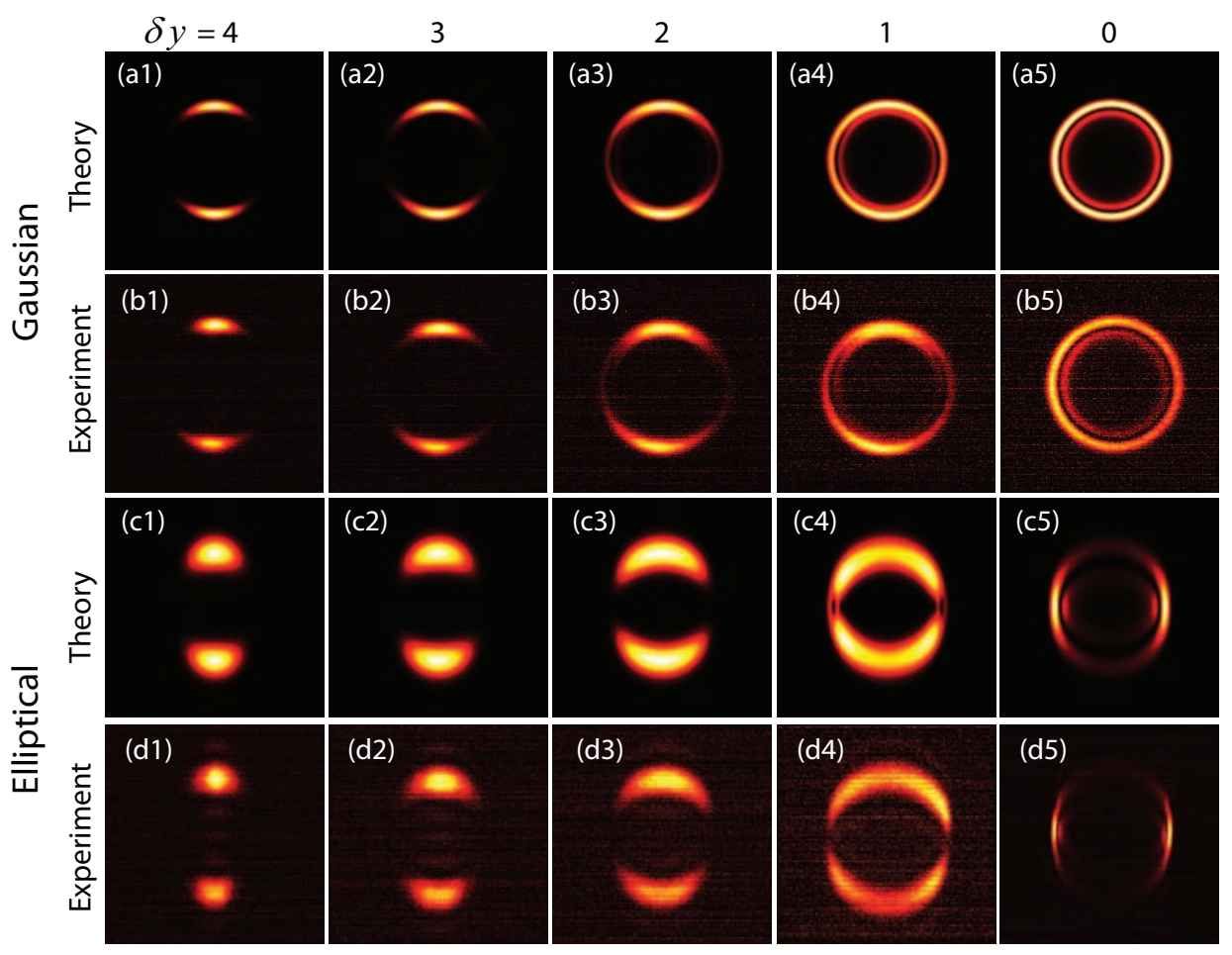

Figure 2.3: Transverse intensity patterns showing the transition from double refraction to conical refraction for a Gaussian input beam (first and second rows) and an elliptical input beam (third and fourth rows) both experimentally (second and fourth rows) and numerically calculated by using Eqs. (2.19)-(2.24) (first and third rows).

The transformation of a Gaussian input beam propagating parallel to one of the optic axes of a biaxial crystal as described by Eq. (2.23) (parallel propagation implies 
$\left.\delta_{x}=\delta_{y}=0\right)$ is the case analyzed in the previous Subsection. When $\delta_{x, y} \neq 0$ double refraction instead of CR is found. Double refraction in uniaxial crystals is associated with the splitting of the input beam into two beams with identical transverse pattern and orthogonal polarizations, corresponding to the ordinary and the extraordinary polarizations. However, in biaxial crystals, a Gaussian input beam propagating out of the optic axes splits into two azimuthal sectors placed at diametrically opposite positions of the otherwise expected CR ring, provided that the angular propagation deviation with respect to the optic axis is small. Only when $\delta_{x, y} \gg 1$ the output split beams preserve the input beam's pattern, as in uniaxial crystals. As the beam propagation direction approaches to the optic axis, the split beams occupy a larger azimuthal angle and eventually both interfere, see Figs. 2.3(a3)-(a5). The fact that the two split beams interfere implies that both beams possess regions of non-orthogonal polarizations. For parallel propagation with respect to the optic axes, the interference between both split beams is maximum and the two bright rings with Poggendorff splitting are formed.

For an elliptical input beam, there is a competition between the ellipticity of the shape of the beam and the refraction induced by the crystal. We consider an elliptical beam described by Eq. (2.24) with $a=1, b=0.1$, i.e., with $w_{x}<w_{y}$. Since the misalignment with respect to the optic axis is only along the $y$ direction, the two split beams are expected also to appear in that direction. Due to the non-symmetrical nature of the elliptical beam and the double refraction provided by the biaxial crystal, which induce opposite effects, the two refracted beams for beam propagation out of the optic axis are wider than for the Gaussian input beam case, see Fig. 2.3(c1). As the misalignment of the input beam is reduced, the refracted beams expand along the azimuthal direction and at some point both interfere. For parallel propagation to one of the optic axis, the pattern is formed by two lobes each of which with Poggendorff splitting, see Fig. 2.3(c5). The two lobes are slightly connected between each other but we have checked that the connection points tend to disappear as the ratio of the axes of the ellipse increases. We have additionally checked that there is a continuous evolution of the double-concentric ring structure from Fig. 2.3(a5) into the double-lobe pattern from Fig. 2.3(c5) as the ratio of the axes of the ellipse increases. In the next section we use strongly elliptical beams to give a formalism capable to predict the transformation of plane waves in CR.

\subsection{Wave-vector and polarization description of conical re- fraction}

Up to this point, we have shown the diffractive theory of $\mathrm{CR}$ and we have demonstrated that it is capable to predict light propagation through biaxial crystals. However, the resulting equations are involved and their computational cost is high. Additionally, they do not offer intuition about the CR pattern obtained for an arbitrary beam. In this section we present a simple analytical formulation capable of predicting the transverse intensity pattern at the focal plane after a light beam propagates along or near the optic 
axis of a biaxial crystal. We experimentally address this by analyzing the wave-vector and polarization dependence of the $\mathrm{CR}$ phenomenon and present a phenomenological formulation that easily describes refraction of a bundle of plane-waves passing through a biaxial crystal. Since the two bright rings of CR have the same polarization distribution, in what follows we will neglect Poggendorff splitting and consider a single bright ring. Our experimental scheme is based on the propagation of elliptical beams (EBs) along the optic axis of a biaxial crystal. EBs do not posses continuous cylindrical symmetry, at variance with Gaussian beams typically used in CR experiments. The approach follows the transformation law pointed out by Loiko et al. [73] for CR filtered beams, which differs from the well known Malus law for double refraction in uniaxial crystals.

\subsubsection{Conical refraction of spatially anisotropic beams revisited}

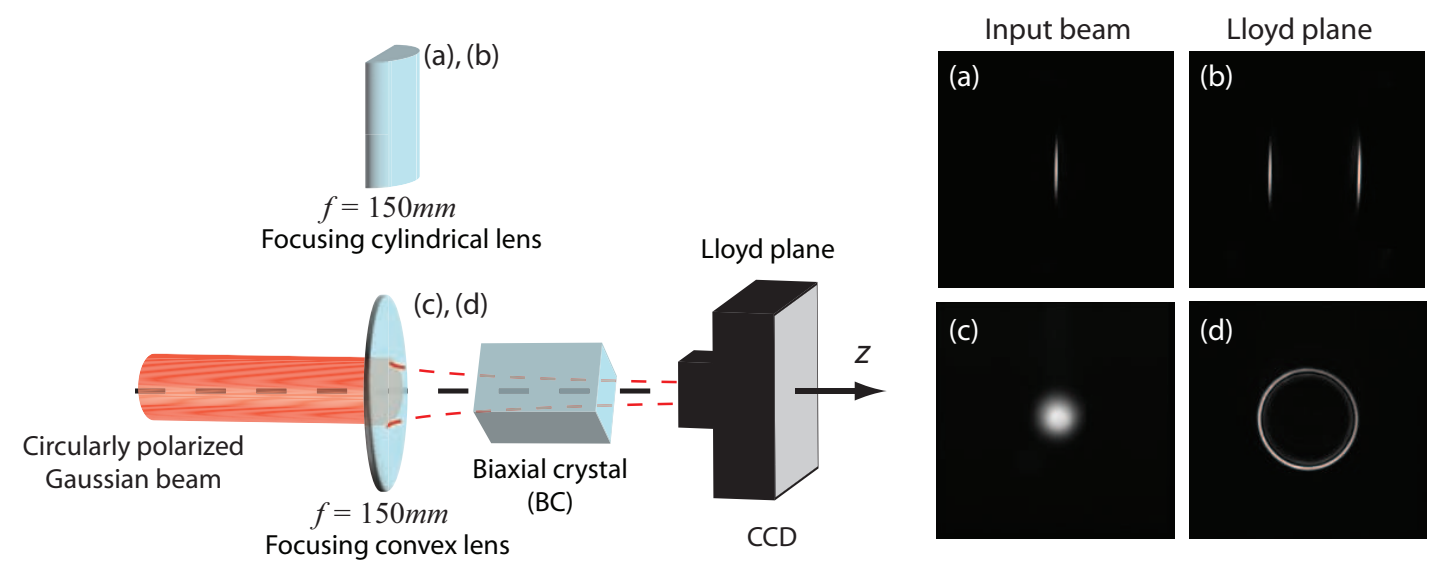

Figure 2.4: Experimental set-up of light refraction along the optic axis of a biaxial crystal (BC): the input beam is focused by a lens and passes through the BC. The refraction pattern is obtained at the focal plane of the system, which coincides with the focal plane. (a) An input Gaussian beam focused with a spherical lens (focal length of $150 \mathrm{~mm}$ ) yields the well-known ring of CR (c) at the focal plane of the system. (b) An elliptical beam (EB) beam, obtained with a cylindrical lens (focal length of $150 \mathrm{~mm}$ ) that focuses in the horizontal direction, yields the the double refraction pattern shown in figure (d).

CR has been mostly reported for input beams with intensity pattern possessing continuous cylindrical (rotation) symmetry around the propagation axis. This has been motivated by Hamilton's prediction of the CR within the ray optics description, where rays are always associated with cylindrically symmetric collimated beams. Real beams of finite size can be modeled as a bundle of rays with propagation directions isotropically distributed around the beam axis. On the one hand, according to the modified Hamilton theory [74, CR can be interpreted as a wave-vector and polarization dependent type of double refraction, when each ray of the input bundle refracts into two rays at the 
entrance crystal surface and all refracted rays form a cone. In this process, the two refracted rays go to opposite points on the $\mathrm{CR}$ ring. On the other hand, it has been also demonstrated that in CR the transverse wave-vector components are conserved [74]. This means that all input rays (plane waves) with wave-vectors confined in a certain plane defined by an azimuthal angle $\phi$ refract to a plane with the same azimuthal angle at the focal plane, i.e., into two opposite points on the CR ring.

Therefore, double refraction along the optic axis of a biaxial crystal and, in particular, the wave-vector and polarization dependence of the CR, can be studied with linearly polarized beams formed by plane waves whose wave-vectors are confined in a certain plane. Such beams are known as cylindrical beams and EBs provide their finite size approximation. The latter ones can be obtained from collimated Gaussian beams focused by a cylindrical lens. In Fig. 2.4 we compare the transverse pattern obtained at the focal plane for a Gaussian and an elliptical input beam. For a Gaussian input beam, the transverse pattern behind the crystal is formed by the well known pair of bright rings with Poggendorff splitting, see Fig. 2.4(d). In contrast, an EB passing through a biaxial crystal does not generate the $\mathrm{CR}$ ring, but splits into two beams (in case of circularly polarized input beams) oppositely placed along the otherwise expected CR ring and tangent to it, see Fig. 2.4(b). The form and orientation of the refracted beams resemble the transverse intensity pattern and orientation of the input EB. Below, using EBs we show how phenomenological laws that describe double refraction along the optic axis in biaxial crystals can be deduced.

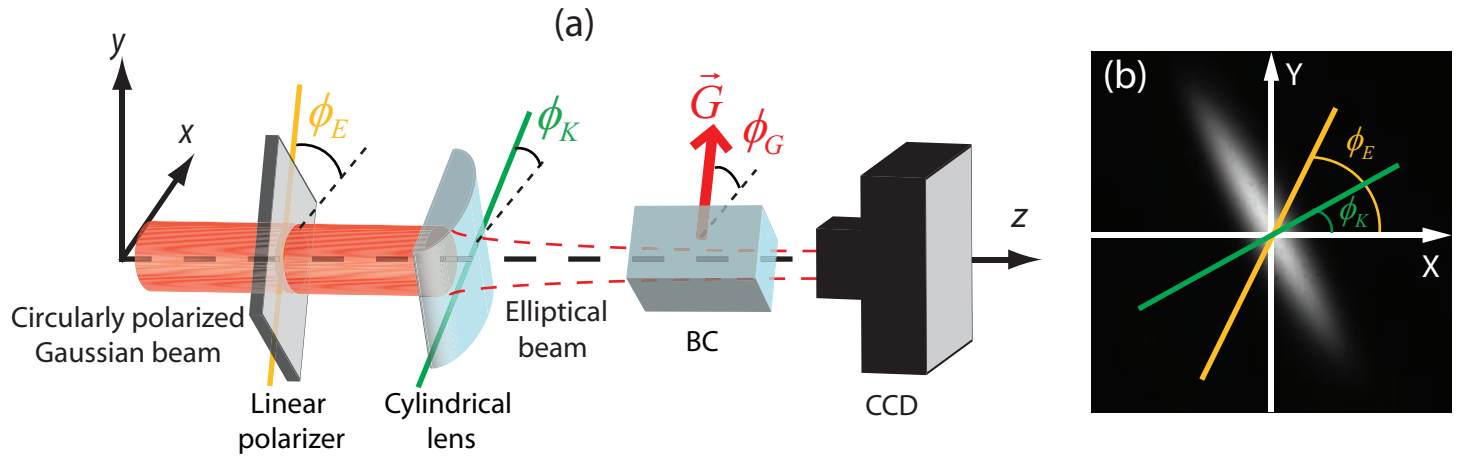

Figure 2.5: (a) An elliptical beam with linear polarization is generated when a circularly polarized collimated Gaussian beam passes through the linear polarizer (with polarization plane given by azimuthal angle $\phi_{E}$ ) and is focused by a cylindrical lens (focal length of $150 \mathrm{~mm}$ ), which determines the wave-vectors plane (given by azimuthal angle $\phi_{K}$ ). The resulting patterns are captured by a CCD camera at the Lloyd plane behind the BC. The orientation of the crystal is characterized by the orientation of the plane of its optic axes (given by azimuthal angle $\phi_{G}$ ). (b) Elliptical beam at the focal plane of the lens when the $\mathrm{BC}$ is removed. The beam is parameterized by the azimuthal angles $\phi_{E}$ and $\phi_{K}$ related to the polarization and wave-vector planes, respectively. All angles are measured from the horizontal $x$-axis of the laboratory system of coordinates. 


\subsubsection{Transformation rules of conical refraction}

In this subsection, we present the position and the relative intensity of the two refracted beams produced when an input EB propagates along the optic axis of a BC. The experimental set-up shown in Fig. 2.5(a) is used. The initial circularly polarized Gaussian beam with waist radius of $w=1 \mathrm{~mm}$ is obtained from a $640 \mathrm{~nm}$ diode laser coupled to a monomode fiber. Then a linear polarizer is introduced to fix the polarization plane in a well defined direction. The resulting linearly polarized beam is focused by a cylindrical lens of $150 \mathrm{~mm}$ focal length with its flat face oriented strictly perpendicular to the beam propagation direction. The cylindrical lens only focuses the Gaussian beam in one direction, so that it transforms its transverse circular shape to an elliptical one with a ratio $3 / 100$ of the semi-axes of the ellipse. As a consequence, the divergence of the generated EB is different along the focused and unfocused directions ( $w_{f}=30 \mu \mathrm{m}, \theta_{f}=$ $6.8 \mathrm{mrad} ; w_{u f}=1000 \mu \mathrm{m}, \theta_{u f}=0.2 \mathrm{mrad}$, where $f$ and $u f$ subscripts refer to focused and unfocused directions, respectively). The EB is characterized by its polarization plane, represented by the azimuthal angle $\phi_{E}$, and by its plane of wave-vectors (or $K$ plane), represented by azimuthal angle $\phi_{K}$; see Fig. 2.5(b). Different EBs are obtained by rotating either the cylindrical lens or the linear polarizer. The $\operatorname{KGd}\left(\mathrm{WO}_{4}\right)_{2} \mathrm{BC}$ is $28 \mathrm{~mm}$ and, therefore, $R_{0}=475 \mu \mathrm{m}$. Both the orientation of the optic axis of the $\mathrm{BC}$ and the cylindrical lens are well controlled in the $\theta$ and $\varphi$ directions in 3D spherical coordinates by a micrometer positioning system. The resulting pattern is captured by a CCD camera at the focal plane behind the BC.

\section{Position of the refracted beams}

Now we will experimentally deduce the lateral shift of the refracted beams for EBs propagated through a BC. In Fig. 2.6 two series of images present the transverse intensity pattern at the focal plane recorded varying either $\phi_{E}\left(\phi_{K}=0^{\circ}\right)(\mathrm{b})$ or $\phi_{K}\left(\phi_{E}=0^{\circ}\right)$ (c) from 0 to $157.5^{\circ}$ in $22.5^{\circ}$ intervals, when the crystal orientation remains fixed at $\phi_{G}=0^{\circ}$. The geometric center of the refracted beams coincides with the center of the otherwise expected CR ring, since it is shifted from the position of the initial EB by the vector G. This shift is shown schematically in Fig. 2.6(a), and it is subtracted in Fig. 2.6(b) and Fig. 2.6(c).

From Fig. 2.6 it is clear that in a local frame with origin at the ring center, the positions of the two refracted beams do not depend on the input beam polarization, $\phi_{E}$, but rotate linearly with $\phi_{K}$. Moreover, the azimuthal angles $\phi_{ \pm}$of the refracted beams are defined by the wave-vector plane, $\phi_{K}$, of the input EB, namely,

$$
\phi_{+}=\phi_{K}, \phi_{-}=\phi_{K}+\pi
$$

Since $\phi_{K}$ and $\phi_{K}+\pi$ describe the same wave-vector plane, the latter expressions mean conservation of the $K$-planes of wave-vectors. Rotation of the crystal, i.e., change of $\phi_{G}$, does not affect the angles $\phi_{ \pm}$, but it affects the position of the center of the refracted 
(a)

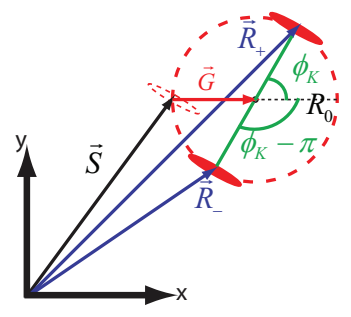

(b)
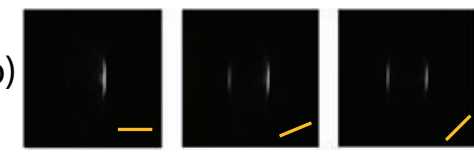

$\phi\left(^{\circ}\right)=0$

22.5

45
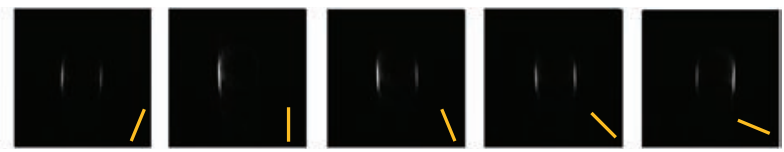

67.5

112.5

(c)
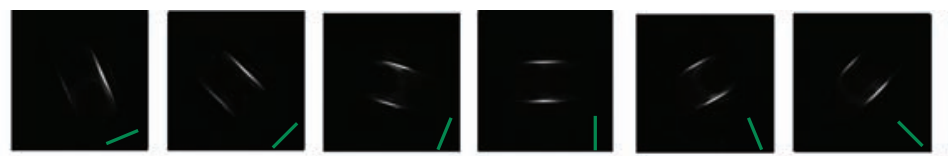

157.5

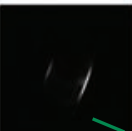

Figure 2.6: Transverse intensity patterns obtained after rotating (b) the polarizer, i.e. varying $\phi_{E}$, or (c) cylindrical lens, i.e. varying $\phi_{K} \cdot \phi$ means $\phi_{E}$ in (b) and $\phi_{K}$ in (c) and it is varied in the range $\left[0^{\circ}, 157.5^{\circ}\right]$ with steps of $22.5^{\circ}$, while $\phi_{G}=0^{\circ}$. Yellow (b) and green (c) lines at the bottom right corner indicate the polarization $E$-plane (b) and the wave-vector $K$-plane (c), respectively. (a) Splitting of an input EB beam (dashed ellipse), where $\mathbf{R}_{ \pm}$denote the position of the two output refracted beams at the Lloyd plane. Dashed ring in (a) denotes the otherwise expected CR in case of input beam of Gaussian profile.

beams and redistributes the intensity between the refracted beams as it will be shown in the next subsection. Summarizing, in the $x y$ laboratory coordinates, the positions of the refracted beams $\mathbf{R}_{ \pm}$at the focal plane can be written as follows:

$$
\mathbf{R}_{ \pm}\left(\phi_{K}\right)=\mathbf{S}\left(\phi_{K}\right)+\mathbf{G} \pm R_{0} \mathbf{u}\left(\phi_{K}\right)
$$

where $\mathbf{u}\left(\phi_{K}\right) \equiv\left(\cos \left(\phi_{K}\right), \sin \left(\phi_{K}\right)\right)$. $\mathbf{S}$ denotes the position at the focal plane where the initial EB would be focused in the absence of the BC, see Fig. 2.6(c). In other words, the two refracted beams are located at diagonal positions of the otherwise expected CR ring. EBs obtained from the same Gaussian beam have the same initial position $\mathbf{S}$, i.e., $\mathbf{S}$ does not depend on $\phi_{E, K, G}$ in this case. As a final comment, Eqs. (2.26) generalize the geometrical approach given in Ref. [74].

\section{Relative intensity distribution of the refracted beams}

Now we report how the intensity of the input EB is distributed between the two refracted beams. In the experiments, three parameters can be varied independently: $\phi_{G}, \phi_{E}$ and $\phi_{K}$, associated to the crystal orientation represented by $\mathbf{G}$ and to the 
polarization and wave-vectors planes of the incident EBs. Below we show that only one combination of these angles governs the relative intensity distribution between the two refracted beams. With this purpose, we have repeated the experiments shown in Fig. 2.6(b) and Fig. 2.6(c) for different orientations of the BC.

(a)

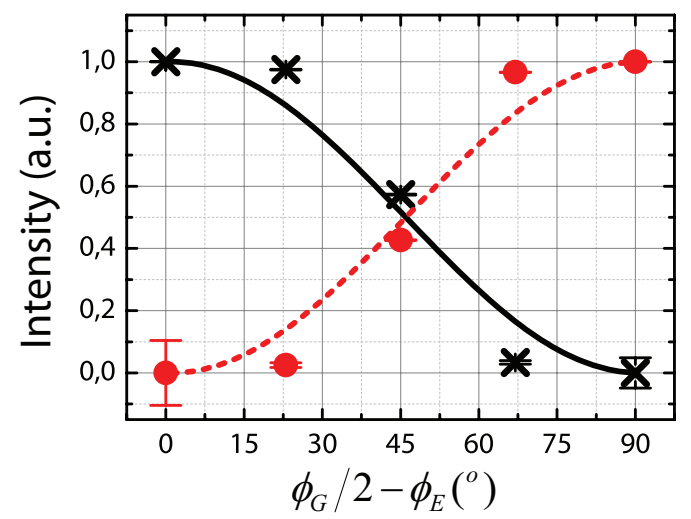

(b)

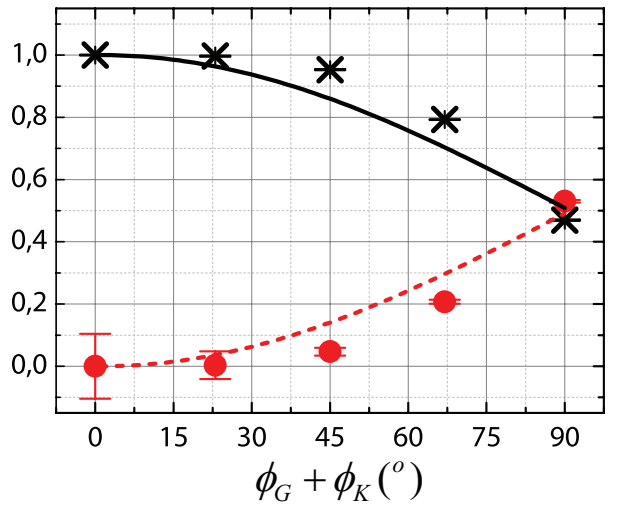

Figure 2.7: Normalized intensities of the two refracted beams after the splitting of the elliptical beam as a function of the variation of the parameters (a) $\phi_{G} / 2-\phi_{E}$ $\left(\phi_{K}=0^{\circ}\right)$ or $(\mathrm{b}) \phi_{G}+\phi_{K}\left(\phi_{E}=0^{\circ}\right)$. Black solid $\left(I_{+}\right)$and red dashed $\left(I_{-}\right)$curves show the analytical fitting given by Eqs. (2.31), while symbols represent the corresponding experimental data. The error in angle measurements is $\pm 0.5^{\circ}$.

Symbols (black crosses and red circles) in Fig. 2.7(a) Fig. 2.7(b) show the corresponding experimental results for the intensities $I_{ \pm}$of the two refracted beams normalized with respect to the intensity of the incident beam. Black solid and red dashed curves represent their analytical fittings given by the following expressions:

$$
\begin{gathered}
I_{+}\left(\phi_{E}, \phi_{G}, \phi_{K}=0\right)=I_{0} \cos ^{2}\left(\frac{\phi_{G}}{2}-\phi_{E}\right) ; \\
I_{-}\left(\phi_{E}, \phi_{G}, \phi_{K}=0\right)=I_{0} \sin ^{2}\left(\frac{\phi_{G}}{2}-\phi_{E}\right), \\
I_{+}\left(\phi_{E}=0, \phi_{G}, \phi_{K}\right)=I_{0} \cos ^{2}\left(\frac{\phi_{G}}{2}+\frac{\phi_{K}}{2}\right) ; \\
I_{-}\left(\phi_{E}=0, \phi_{G}, \phi_{K}\right)=I_{0} \sin ^{2}\left(\frac{\phi_{G}}{2}+\frac{\phi_{K}}{2}\right) .
\end{gathered}
$$

$I_{+}$and $I_{-}$are the intensities of the beams refracted at angles $\phi_{+}$and $\phi_{-}$and located diagonally at the both ends of the CR ring at positions $R_{+}$and $R_{-}$respectively, following Eq.(2.26). Eqs. (2.28) and (2.30) can be rewritten in a unified simple form, giving the next formula for the relative intensity distribution between the two refracted beams with only one governing parameter $\omega$ :

$$
I_{+}=I_{0} \cos ^{2}\left(\frac{\omega}{2}\right), \quad I_{-}=I_{0} \sin ^{2}\left(\frac{\omega}{2}\right), \omega \equiv \phi_{G}-\phi_{\chi}, \quad \phi_{\chi}=2 \phi_{E}-\phi_{K} .
$$


From Eq. (2.31) it follows that, with respect to the relative energy distribution the only significant parameters of the input EB is $\phi_{\chi}$. Therefore, the intensity splitting under CR can be expressed in terms of the difference between the parameter $\phi_{\chi}$ and the orientation of the $\mathrm{BC}$, given by $\phi_{G}$.

Eqs. (2.26) and (2.31) constitute the Transformation Rules of Conical Refraction. For different polarization of the input beams they allow explaining the ratio of intensities for any pair of diagonally opposite points of the CR ring. These results agree with experimental observations that the complete CR ring appears only for beams with azimuthally continuous symmetric distribution of wave-vectors. As a proof of usefulness of the formalism introduced in this work, in the next subsection we apply the derived transformation rules to an axicon input beam.

\subsubsection{Application of the transformation rules of $\mathrm{CR}$ to an axicon beam}

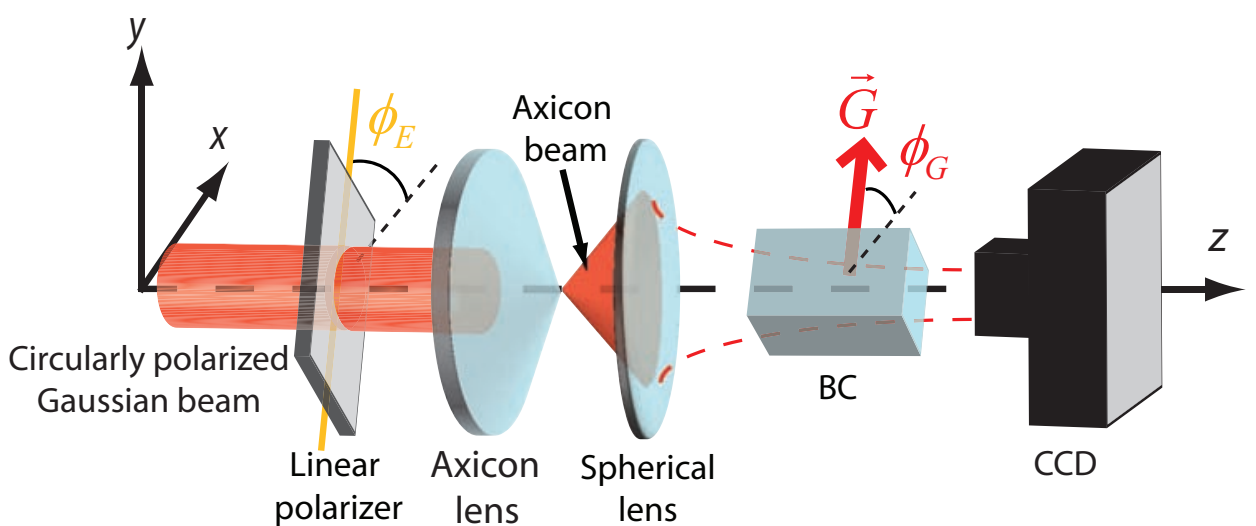

Figure 2.8: Experimental set-up for axicon beam propagation along the optic axis of a biaxial crystal. The axicon lens (apex angle of $179.5^{\circ}$ ) generates a conical beam from an input linearly polarized Gaussian beam which is then focused by a spherical lens (focal length of $150 \mathrm{~mm}$ ) along the optic axis of the $\mathrm{BC}$.

The experimental set-up is sketched in Fig. 2.8. A linearly polarized conical beam is prepared when an initial circularly polarized Gaussian beam passes through a linear polarizer and an axicon lens. The axicon beam is focused by a spherical lens into the BC. The resulting pattern is captured with a CCD camera at the focal plane. As in previous experiments, the orientation of the optic axis of the $\mathrm{BC}$, the spherical lens and the axicon lens are well controlled in the $\theta$ and $\varphi$ directions in 3D spherical coordinates by a micrometer positioning system. Each infinitesimally thin azimuthal sector of the axicon lens characterized by azimuthal angle $\phi$ forms a thin prism that produces a wave with particular wave-vector whose transverse projection comprises an angle $\phi_{k}=\phi$. The 
axicon lens generates, therefore, a continuous collection of beams with $\phi \in[0,2 \pi)$. After the axicon, the refracted beams, following Snell law, have the same inclination angle $\theta_{0}$ with respect to the $z$-axis. At the focal plane of the lens they form a ring such that each point can be characterized by wave-vector plane, $\phi_{k}$, and polarization plane, $\phi_{E}$. In other words, each point of the axicon ring is an EB. In this case all these EB have their polarization plane fixed at $\phi_{E}$ and their wave-vector plane $\phi$ is varying continuously along the ring as shown in Fig. 2.9(a). Behind the BC, the refraction pattern can be calculated by applying Eq. (2.26) to every point of the input axicon annular beam taking into account the initial positions as given by $\mathbf{S}(\phi)=R_{a x}(\cos \phi, \sin \phi)$ (where $R_{a x}$ is the radius of the axicon light ring). Therefore, from Eq. (2.26) one can obtain the refracted pattern for an axicon beam:

$$
\mathbf{R}_{ \pm}(\phi)=\mathbf{G}+\left(\left|R_{a x}\right| \pm\left|R_{0}\right|\right) \mathbf{u}(\phi) .
$$

These expressions, with $\phi$ scanned from 0 to $2 \pi$, parametrize two concentric rings with radii $R_{a x} \pm R_{0}$ laterally shifted by $\mathbf{G}$ relatively to the axicon ring axis. The intensity distribution is calculated from Eq. (2.31). All points of the incident axicon beam have the same intensity, which is distributed between the two refracted rings as follows:

$$
I_{+}=I_{0} \frac{R_{0}}{R_{+}} \cos ^{2}\left(\frac{\phi-\phi_{0}}{2}\right), \quad I_{-}=I_{0} \frac{R_{0}}{R_{-}} \sin ^{2}\left(\frac{\phi-\phi_{0}}{2}\right),
$$

where we have taken $\omega=\phi-\phi_{0}$ being $\phi_{0} \equiv 2 \phi_{E}-\phi_{G}$ a constant parameter. In addition, since both rings have different radii, normalization factors $R_{0} / R_{ \pm}$have been introduced to $I_{ \pm}$to assure energy conservation.
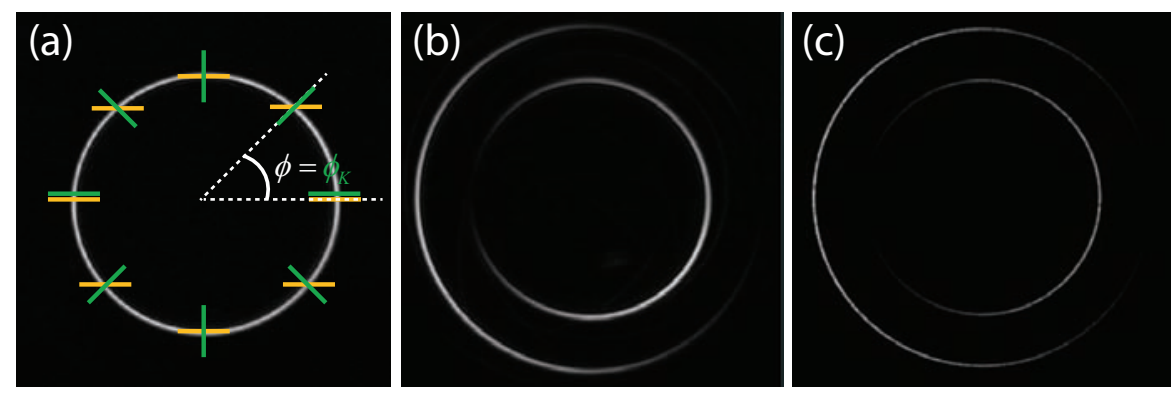

Figure 2.9: Refraction of linearly polarized axicon annular beam along optic axis of a biaxial crystal. (a) Schematic representation of linearly polarized axicon annular beam with each point characterized by the azimuthal angles $\phi_{k}=\phi$ (short green lines) and all of them have the same polarization plane $\phi_{E}$ (short orange lines). (b) Experimentally observed transverse intensity pattern at the focal plane consisting of two concentric rings when a linearly polarized axicon beam with $\phi_{E}=0$ propagates along the optic axis of the biaxial crystal with $\phi_{G}=0$. (c) Corresponding theoretical simulation from Eqs. (2.32) and (2.33).

Fig. 2.9(a) shows the intensity pattern of the axicon beam with its polarization and $K$-plane distribution. The experimental refraction pattern behind the $\mathrm{BC}$ is shown in 
Fig. 2.9(b). The pattern is formed by two concentric rings oppositely polarized, with polarization distribution analogous to that one obtained in a cascaded CR configuration 33. Polarization of the ring points with minimum (zero) and maximum intensities are orthogonal and parallel respectively to that of the input axicon beam. Fig. 2.2. (c) presents the theoretical prediction of the light refraction pattern from Eqs.(2.32) and (2.33). Comparison between Fig. 2.9(b) and Fig. 2.9)(c) shows that the theoretical prediction and the experiment (see Fig. 2.9(b)) are in complete agreement.

\subsection{Dual-cone model of conical refraction}

\subsubsection{Dual-cone theory and its relation to the wave-vector interpreta- tion}

Following the dual-cone model of $\mathrm{CR}$, which can be obtained by re-arranging the Belsky-Khapalyuk-Berry $B_{0}$ and $B_{1}$ equations i.e., Eqs. (2.12) and (2.13), for a uniformly polarized input light beam with axially symmetric intensity distribution, the electric field behind the biaxial crystal can be represented as a sum of two CR cones $\mathbf{C}_{ \pm}(\rho, \varphi, Z)$ :

$$
\begin{aligned}
\mathbf{E}(\rho, \varphi, Z) & =\mathbf{C}_{+}(\rho, \varphi, Z)+\mathbf{C}_{-}(\rho, \varphi, Z), \\
\mathbf{C}_{q}(\rho, \varphi, Z) & =\sum_{q, s= \pm} A_{q s}(\rho, Z) \mathbf{e}_{\mathbf{s}}(\varphi)\left(\mathbf{e}_{\mathbf{s}}(\varphi) \cdot \mathbf{e}_{\mathrm{in}}\right), \\
A_{q s}(\rho, Z) & =\frac{1}{2} \int_{0}^{\infty} d k a(k) k e^{-i Z \frac{k^{2}}{4}} e^{i q \rho_{0} k}\left(J_{0}(k \rho)-i q s J_{1}(k \rho)\right), \\
\mathbf{e}_{+}(\varphi) & =\left[\cos \left(\frac{\varphi}{2}\right), \sin \left(\frac{\varphi}{2}\right)\right] \\
\mathbf{e}_{-}(\varphi) & =\left[\sin \left(\frac{\varphi}{2}\right),-\cos \left(\frac{\varphi}{2}\right)\right] .
\end{aligned}
$$

where dot in Eq. (2.35) means scalar product of two vectors and $a(k)$ is given by Eq. (2.8). $\mathbf{e}_{ \pm}$describe the CR polarization basis. For a circularly polarized input beam with helicity $\sigma= \pm$, the two CR cones $\mathbf{C}_{q}^{\sigma}(q= \pm)$ and the total CR electric field $\mathbf{E}=\left[E_{x}, E_{y}\right]$ can be written as follows:

$$
\begin{aligned}
\mathbf{C}_{q}^{\sigma} & =\frac{e^{i \sigma \varphi / 2}}{\sqrt{2}}\left(A_{q+} \mathbf{e}_{+}-i \sigma A_{q-} \mathbf{e}_{-}\right), \\
E_{x}^{\sigma} & =\frac{e^{i \sigma \varphi / 2}}{\sqrt{2}}\left\{\left(A_{++}+A_{-+}\right) \cos \left(\frac{\varphi}{2}\right) i \sigma\left(A_{+-}+A_{--}\right) \sin \left(\frac{\varphi}{2}\right)\right\}, \\
E_{y}^{\sigma} & =\frac{e^{i \sigma \varphi / 2}}{\sqrt{2}}\left\{\left(A_{++}+A_{-+}\right) \sin \left(\frac{\varphi}{2}\right) i \sigma\left(A_{+-}+A_{--}\right) \cos \left(\frac{\varphi}{2}\right)\right\},
\end{aligned}
$$

where $A_{q s}$ with $q, s= \pm$ is described by Eq. (2.36).

From Eqs. (2.40) and (2.41) it follows that each of the two CR cones can be represented as a decomposition into the two unit vectors $\mathbf{e}_{ \pm}$of the CR basis. Note that $\mathbf{e}_{ \pm}$ 
are orthogonal to each other at any point in 3D space. They are non-homogeneously polarized, in contrast to the well known polarization basis of linearly and circularly polarized states that have homogeneous polarization distribution in 3D space. The CR polarization basis is also different from the well known inhomogeneous polarization basis formed by radially and azimuthally polarized states. In particular, the unit vectors $\mathbf{e}_{ \pm}$ are rotated by $180^{\circ}$ along any closed loop around the center of coordinates normally associated with the geometric center of the light beam.

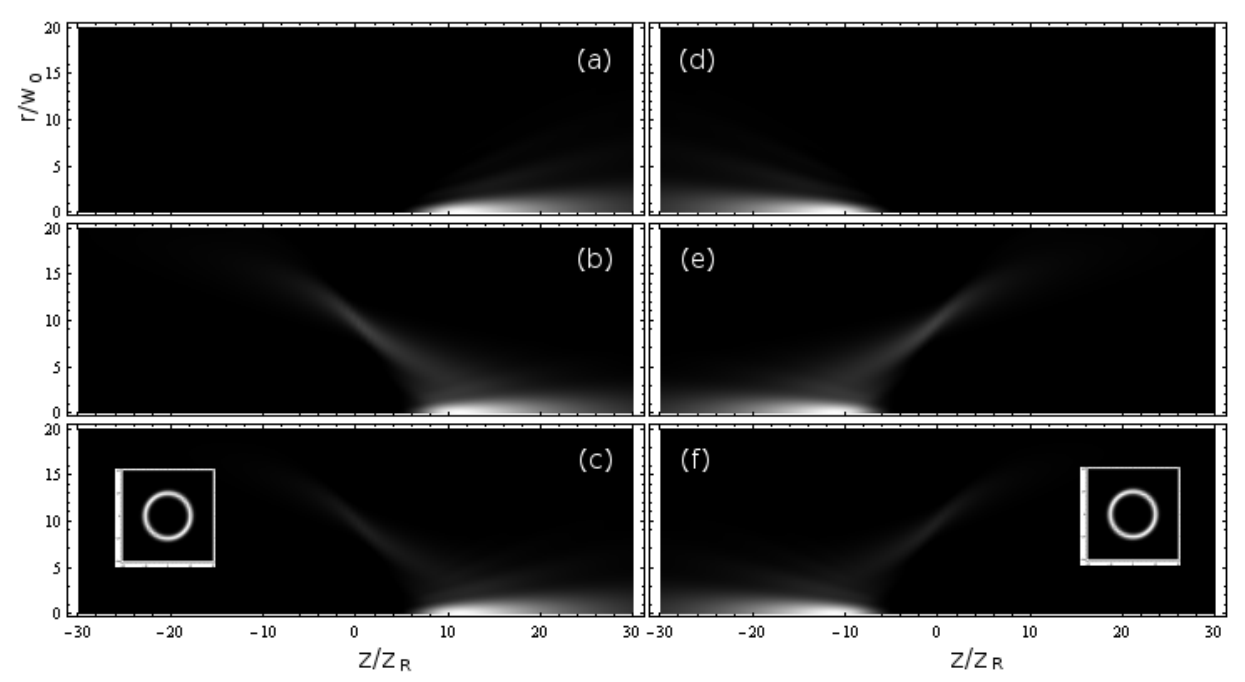

Figure 2.10: Decomposition amplitudes $A_{q s}$ of the CR cones $\mathbf{C}_{q=+}(\mathrm{a}, \mathrm{b})$ and $\mathbf{C}_{q=-}$ $(\mathrm{d}, \mathrm{e})$ onto the CR polarization basis of $\mathbf{e}_{s=+}(\mathrm{a}, \mathrm{d})$ and $\mathbf{e}_{s=-}(\mathrm{b}, \mathrm{e})$ for an input beam with fundamental Gaussian transverse profile. Intensity evolution of the CR cones $\mathbf{C}_{+}$ and $\mathbf{C}_{-}$are presented in figures (c) and (f), respectively. Their corresponding transverse profiles at the focal plane are presented in the insets. $\rho_{0}=10$.

Note that in the general case, decompositions of beams on the CR polarization basis and on the CR cones are different. Each CR cone has two components in the CR polarization basis as demonstrated in Fig. 2.10. The amplitudes of this decomposition are strongly separated in space. The separation point is associated with the CR cone vertexes, i.e., the Raman spots. The $\mathbf{C}_{+}$and $\mathbf{C}_{-}$cones have smallest diameter at the Raman spot behind and before the focal focal plane, respectively. As shown in Fig. 2.10, for the $\mathrm{CR}$ cone $\mathbf{C}_{+}$the polarization amplitude $A_{++}$becomes negligible after the Raman spot behind the focal focal plane, while $A_{+-}$is negligible before the same Raman spot. For the CR cone $\mathbf{C}_{-}$the contributions of amplitudes in the CR polarization basis are opposite, i.e., $A_{-+}\left(A_{--}\right)$becomes negligible before (after) the vertex of the corresponding $\mathrm{CR}$ cone $\mathbf{C}_{-}$. Therefore, $\mathrm{CR}$ cones $\mathbf{C}_{ \pm}$have almost identical polarization distribution between the Raman spots and, consequently, they can interfere with each other, which leads to the double bright ring pattern with the Poggendorff fine splitting at the focal plane previously observed in conical refraction. 
From \pm infinity to the Raman spots the $\mathrm{CR}$ cones $\mathbf{C}_{ \pm}$are predominantly of orthogonal polarization at any spatial point and, therefore, they do not interfere. Moreover, if the polarization distribution of an input beam coincides with one of the CR cones only this CR cone is observed under conical refraction and, therefore, the interference pattern, i.e., the Poggendorff dark ring, disappears at the focal plane of conical refraction as shown theoretically in Fig. 2.10(c) and Fig. 2.10(f) and demonstrated experimentally in the next subsection. This model reproduces well the features of the CR beam shown in Fig. 2.2 with the add that in this case the beam can clearly be decomposed into two contributions, as shown in the next subsection.

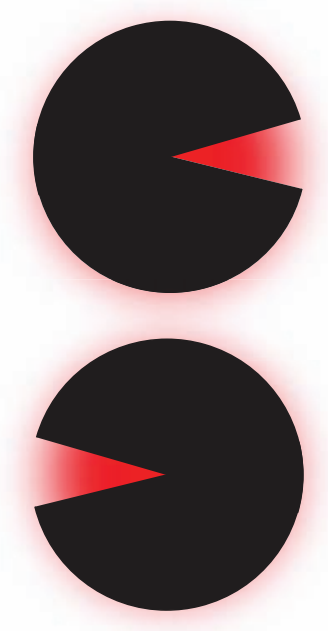

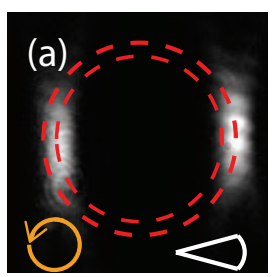

$C P$

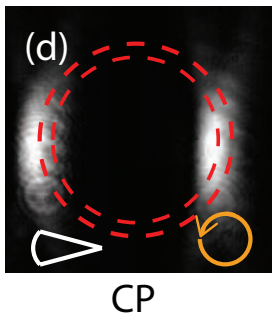

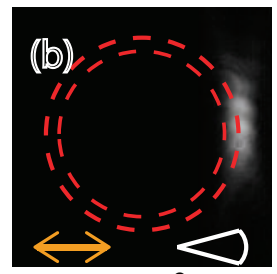

$\operatorname{LP} 0^{\circ}$

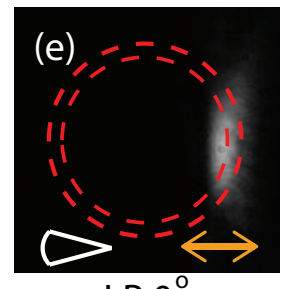

LP $0^{\circ}$

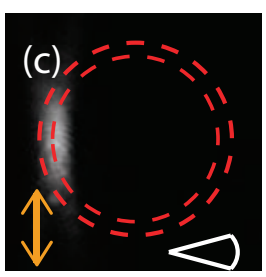

$\operatorname{LP} 90^{\circ}$

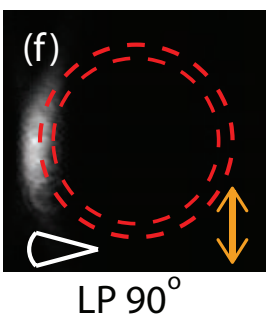

Figure 2.11: Double conical refraction experiment. (a)-(c) are the experimental CR transverse profiles obtained at the focal plane when the input beam is a sector of a Gaussian beam with its wave-vectors mainly contained in a plane at angle $\phi_{k}=0^{\circ}$. Such a beam is obtained by applying an amplitude mask onto the beam, see its sketch at the left side of the corresponding figure. (d)-(f) are the corresponding experimental transverse patterns for $\phi_{k}=180^{\circ}$. In both cases only one refracted beam is obtained by properly choosing the input polarization.

Alternatively, we have shown in the previous section that an input plane-wave with transverse wave-vector $\mathbf{k}_{\perp}=k\left[\cos \left(\phi_{k}\right), \sin \left(\phi_{k}\right)\right]$ refracts into two opposite points in the expected CR rings, after passing through the biaxial crystal. If the polarization of this ray is selected properly, only one point will be observed at the otherwise expected CR ring. Having this idea in mind, let's consider the following experiment: in front of a Gaussian beam we place an angular mask that only transmits one angular sector of the beam, as depicted in the inset of Fig. 2.11(a). If the angular mask has the opening sector at an angle $\phi$, most of the wave-vectors of the transmitted beam posses transverse wave vector $\mathbf{k}_{\text {in }}=k[\cos (\phi), \sin (\phi)]$. In this case, a circularly polarized input beam cut with such an angular mask and passing through the biaxial crystal is transformed into 
two azimuthal sectors at positions $\phi$ and $\phi+\pi$ of the otherwise expected CR rings, represented as dotted lines in Fig. 2.11(a).

However, note that each of the two azimuthal sectors do not belong to the same ring. Therefore, if the polarization of the input beam is fixed to be linear with azimuth at $\Phi=\phi / 2$ or $\Phi=(\phi+\pi) / 2$, after being transformed by the biaxial crystal only one sector of the CR ring will be observed. This is shown in Figs. 2.11(b) and (c) for $\Phi=0$ and $\Phi=\pi / 2$ respectively. If the angular mask is placed with the opened sector at $\phi+\pi$, it will be also transformed into two azimuthal sectors not belonging to the same ring either, see Fig. 2.11(d). Analogously, if the transmitted sector is linearly polarized with azimuth at $\Phi=(\phi+\pi) / 2$ or $\Phi=\phi / 2$, only one azimuthal sector will be observed behind the biaxial crystal, as shown in Figs. 2.11(e) and (f) for $\Phi=0$ and $\Phi=\pi / 2$ respectively. These results indicate that if we consider a Gaussian input beam that possesses a nonuniform polarization mimicking the CR polarization, it is possible to select only one of the two bright rings.

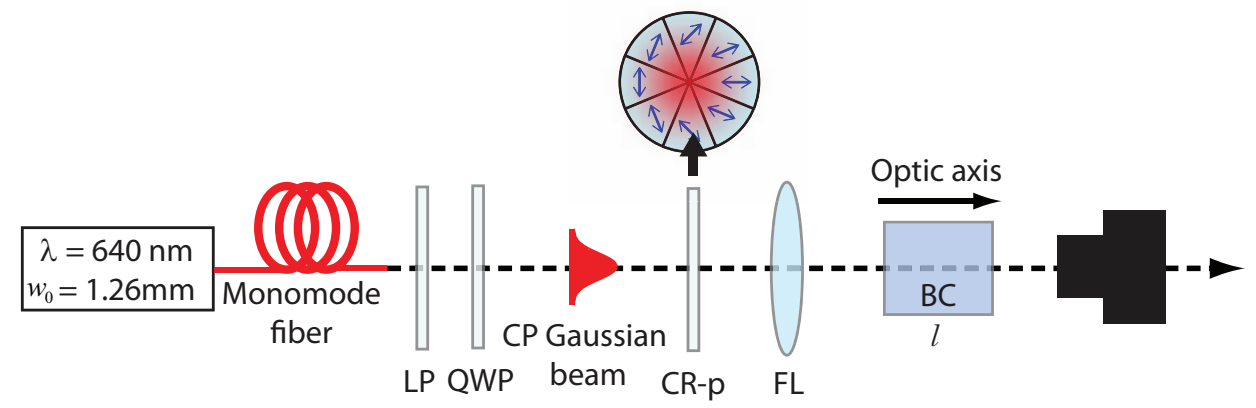

Figure 2.12: Experimental set-up. The input Gaussian beam is obtained from a diode laser coupled to a monomode fiber. Its polarization is fixed to be circular by means of a linear polarizer (LP) and a quarter wave-plate (QWP). A segmented polarizer (CR-p) that can be rotated around the axial direction transforms the Gaussian beam into a non-uniformly polarized beam that mimics the CR polarization azimuthal distribution. The beam is focused (FL) through the biaxial crystal (BC) and the pattern at the focal plane is recorded by a CCD camera.

\subsubsection{Experiments}

To show the dual-cone nature of the CR beam, we have designed a segmented polarizer formed by 8 sectors emulating the CR polarization -for this reason we call it as CR-polarizer. By taking into account the experimental results shown in Fig. 2.11, it is straightforward to deduce that for two appropriate orientations of the CR-polarizer, only one light ring is observed at the CR pattern at the focal plane. In this section we provide experimental proof of this conclusion.

Fig. 2.12 shows our experimental set-up. As input beam, we take a collimated 
linearly polarized Gaussian beam with $w_{0} \approx 1 \mathrm{~mm}$ waist radius obtained from a $640 \mathrm{~nm}$ diode laser coupled to a monomode fiber. The linear polarizer (LP) and the quarter wave-plate (QWP) are used to control the polarization of the input beam and fix it to be circular. The CR-polarizer is placed after the QWP with its center coinciding with the center of the beam, which is focused by the lens ( $200 \mathrm{~mm}$ of focal length) upon the biaxial crystal (BC). To ensure that the vertexes of the CR-polarizer are right at the center of the beam, we use an $X Y$ micro-positioner. Once focused, the beam has a waist radius of $w_{0} \approx 41 \mu \mathrm{m}$.
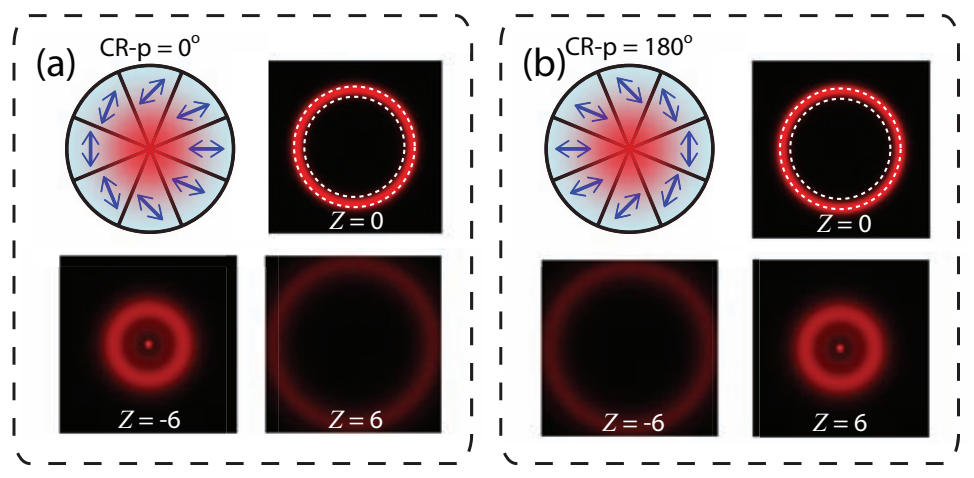
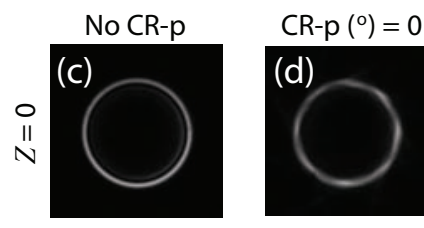

45
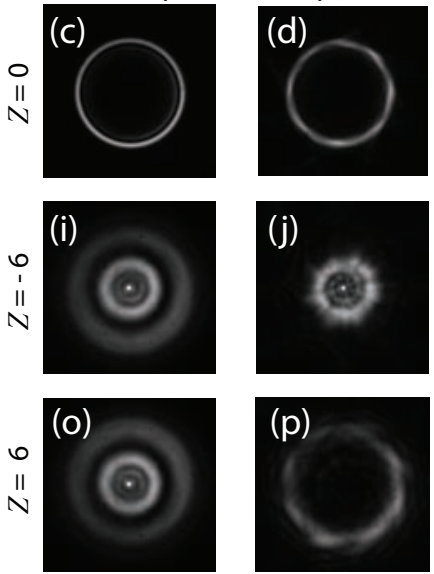
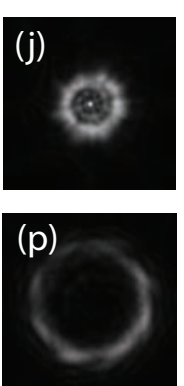
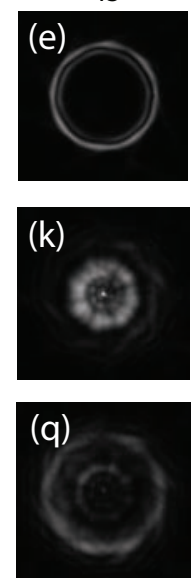

90
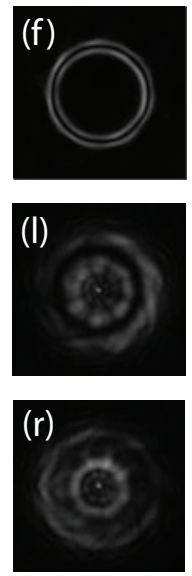

135
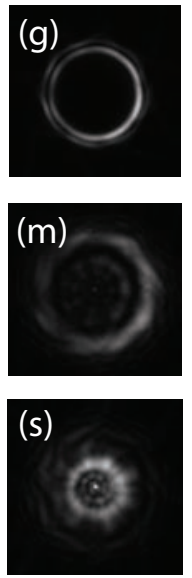

180
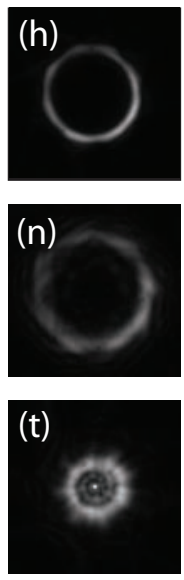

Figure 2.13: (a) and (b) show two particular configurations of the CR-polarizer for which only one CR light ring is obtained. The density plots show the numerically obtained light ring at the focal plane $(Z=0)$, while the dotted concentric rings are the position where the $\mathrm{CR}$ bright rings would appear in the absence of the CR-polarizer. Transverse patterns obtained at $Z= \pm 6$ are also shown. (c)-(h) show the transverse CR pattern at the focal plane for different orientations of the CR-polarizer with respect to the polarization distribution generated by the CR phenomenon [see double arrows in Fig. [2.2(a)]. The characteristic CR bright rings are recovered for $\phi_{\mathrm{CR}-\mathrm{p}}=90^{\circ}$, while for $\phi_{\mathrm{CR}-\mathrm{p}}=0^{\circ}$ or $\phi_{\mathrm{CR}-\mathrm{p}}=180^{\circ}$ only one bright ring is obtained. (i)-(n) and (o)-(t) are the corresponding transverse patterns (demagnified in size around a $30 \%$ ) at $Z=-6$ and $Z=6$, respectively. 
As $\mathrm{BC}$ we use a $\mathrm{KGd}\left(\mathrm{WO}_{4}\right)_{2}$ crystal being $l=28 \mathrm{~mm}$, therefore $R_{0} \approx 475 \mu \mathrm{m}$, and yielding $\rho_{0} \approx 11$. The CCD camera is mounted on a translation stage to record the pattern at different planes along the beam propagation.

The possibility to use the CR-polarizer to observe the dual-cone nature of $\mathrm{CR}$ is reported in Fig. 2.13, Figs. 2.13 (d)-(h) show the CR pattern at the focal plane for rotation of the CR-polarizer at angles $\phi_{\mathrm{CR}-\mathrm{p}}=\left[0^{\circ}, 180^{\circ}\right]$ in steps of $45^{\circ}$. Fig. 2.13(c) is the pattern obtained in the absence of the CR-polarizer. At $\phi_{\mathrm{CR}-\mathrm{p}}=0$ only one light ring is observable. As $\phi_{\mathrm{CR}-\mathrm{p}}$ increases, the intensity of this ring decreases and an inner ring starts to form. At $\phi_{\mathrm{CR}-\mathrm{p}}=90^{\circ}$ the pattern is clearly formed by two light rings. From $\phi_{\mathrm{CR}-\mathrm{p}}=90^{\circ}$ on, the intensity of the outer ring keeps decreasing as the one from the inner increases, until having only one light ring again at $\phi_{\mathrm{CR}-\mathrm{p}}=180^{\circ}$. To provide an even clearer visualization of the effect producing by the rotation of the CR-polarizer, we have carried out the same experiment at $Z= \pm 6$, the planes where the Raman spots starts to appear.
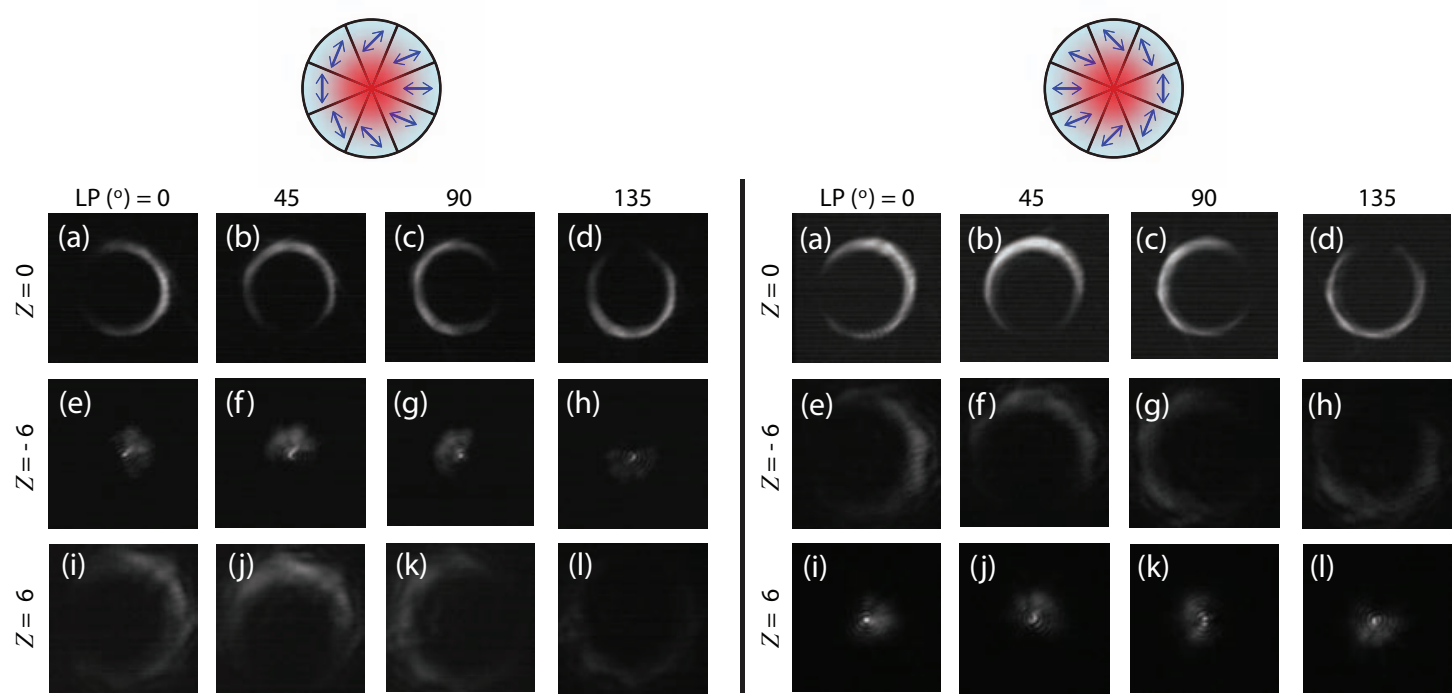

(k)

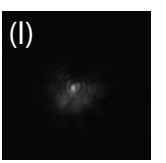

Figure 2.14: CR transverse intensity profiles at the focal plane (first row) and at $Z=-6$ (second row) and $Z=+6$ (third row) for orientations of a linear polarizer (LP) at angles: $\left[0^{\circ}, 45^{\circ}, 90^{\circ}, 135^{\circ}\right]$. Left- and right-hand side set of images correspond to $\phi_{\mathrm{CR}-\mathrm{p}}=0^{\circ}$ and $\phi_{\mathrm{CR}-\mathrm{p}}=180^{\circ}$, respectively.

The results are shown in Figs. 2.13(j)-(n) for $Z=-6$ and in Figs. 2.13(p)-(t) for $Z=6$, being Figs. 2.13)(i) and (o) the pattern obtained in the absence of the CRpolarizer. At $Z=-6$ and for $\phi_{\mathrm{CR}-\mathrm{p}}=0^{\circ}$, the CR pattern only has contributions from the beam center. In contrast, at $\phi_{\mathrm{CR}-\mathrm{p}}=180^{\circ}$ a light ring with no intensity at its center is found. At intermediary angles of the CR-polarizer, contributions of both the central intensity and the light ring are found. At $Z=6$ the $\mathrm{CR}$ pattern obtained for $\phi_{\mathrm{CR}-\mathrm{p}}=0^{\circ}$ is a light ring whereas at $\phi_{\mathrm{CR}-\mathrm{p}}=180^{\circ}$ only intensity at the beam center 
is observed. These results, together with the one presented in Figs. 2.13(d)-(h) indicate that the CR beam can be actually understood as two axially displaced light cones, being the focal plane a mirror symmetry-plane.

It is also necessary to show the state of polarization of the light cones. Fig. 2.14 presents the experimental images of the CR transverse intensity profiles at the focal plane (first row) and at $Z=-6$ (second row) and $Z=+6$ (third row) for different orientations of a linear polarizer (LP) used to analyze the state of polarization of the two cones. Left-hand set of images correspond to $\phi_{\mathrm{CR}-\mathrm{p}}=0^{\circ}$ while right-hand side set of images correspond to $\phi_{\mathrm{CR}-\mathrm{p}}=180^{\circ}$ see top insets. Each set of images correspond to the $C_{-}$and $C_{+}$cones, respectively. The images show two remarkable phenomena: (i) every two diametrically opposite points at the light pattern are orthogonally polarized at any plane, and (ii) the polarization distributions of both light cones are the same.
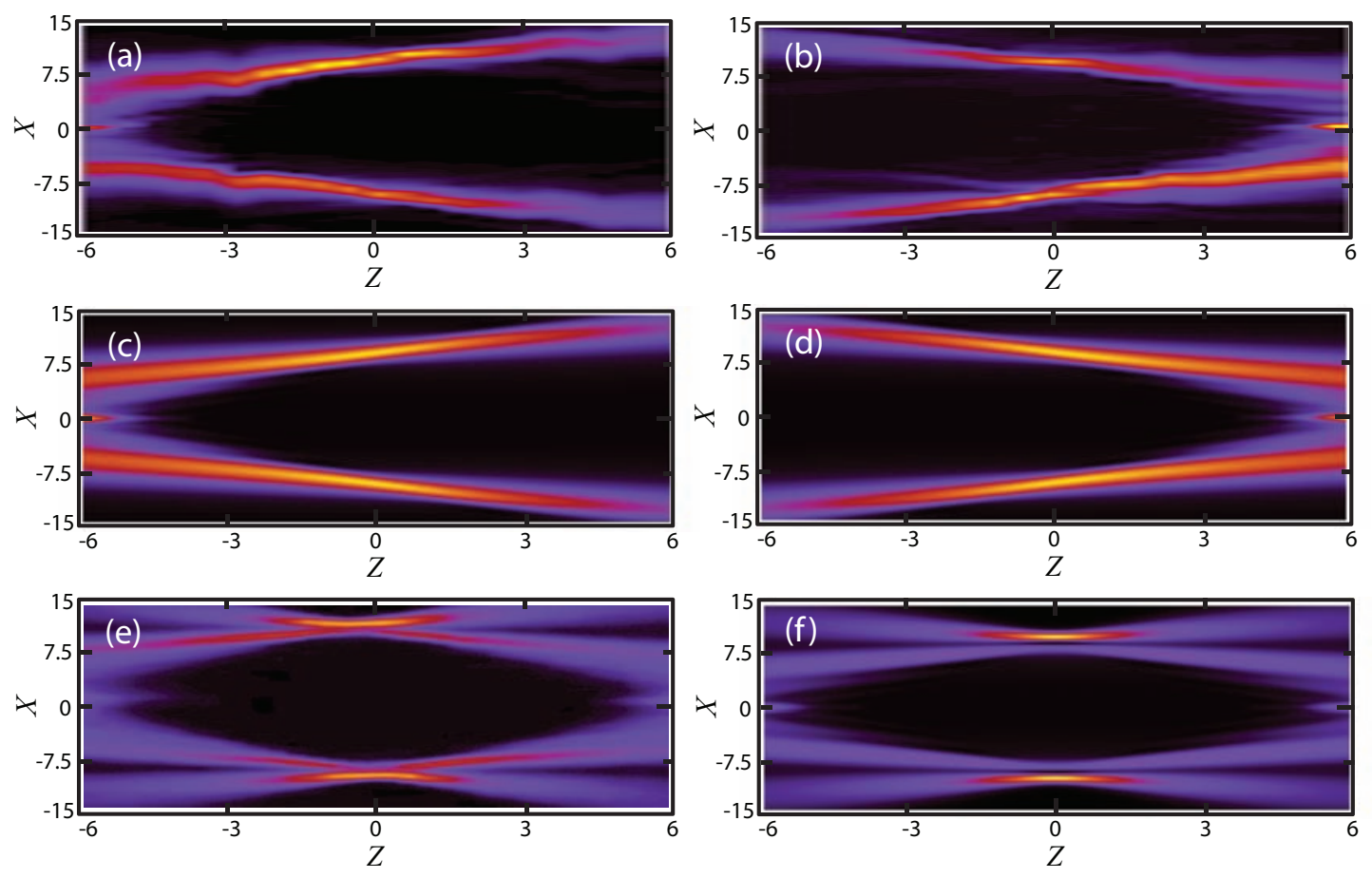

Figure 2.15: Cuts in the $Z-X$ plane (where $X \equiv x / w_{0}$ ) of the beam evolution obtained for two orientations of the CR-polarizer at (a) and (c) $0^{\circ}$, and (b) and (d) $180^{\circ}$, showing the dual-cone nature of the CR phenomenon. The first row presents the experimental measurements obtained by recording the transverse pattern at different propagation planes, while the corresponding numerical simulations obtained using Eqs. (2.40)-(2.41) can be found in second row. (e) and (f) represent, respectively, the experimental and numerical beam propagation with the CR-polarizer being removed from the set-up. $\rho_{0}^{\text {th }}=10, \rho_{0}^{\text {exp }} \approx 11$. Black is null intensity and yellow is maximum intensity.

To observe the free space evolution of the light cones, Fig. 2.15 presents cuts in the $Z_{-}$ 
$X$ plane of the beam evolution of the CR pattern using the CR-polarizer. First row are the experimental results, while second row are the numerical simulations obtained using the dual-cone model, i.e., Eqs. (6)-(2.41). Figs. 2.15(a) (obtained with $\phi_{\mathrm{CR}-\mathrm{p}}=0^{\circ}$ ) and (c) correspond to the $C_{-}$light cone, while Figs. 2.15(b) (obtained with $\phi_{\mathrm{CR}-\mathrm{p}}=180^{\circ}$ ) and (d) correspond to the $C_{+}$light cone.

Fig. 2.15)(e) shows the experimental beam evolution with the CR-polarizer being removed and Fig. 2.15(f) is the corresponding numerical simulation. The experimental images were taken by recording the transverse light pattern at different planes along the axial direction in steps of $5 \mathrm{~mm}$ and then interpolating between them using the software ImageJ. As it can be appreciated, both experiments and numerical simulations are in good agreement, confirming the dual-cone nature of the CR phenomenon.

\subsection{Cascaded conical refraction}
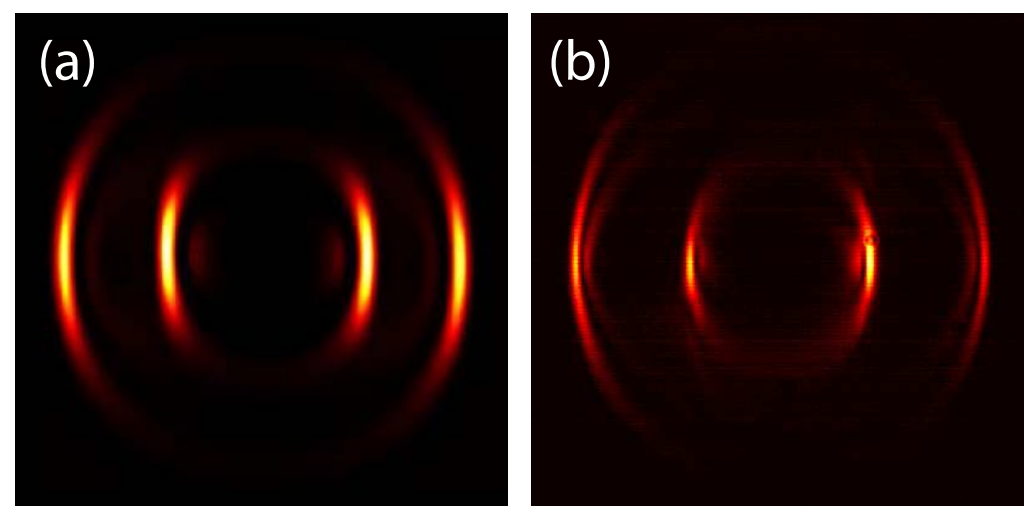

Figure 2.16: Transverse intensity pattern at the focal plane for a cascade of two biaxial crystals with aligned optic axes obtained from an elliptical input beam. (a) Numerical simulations obtained by using Eqs. (2.19) (2.24). (b) Experimental measurements obtained by using two $\mathrm{KGd}\left(\mathrm{WO}_{2}\right)_{4}$ biaxial crystals with lengths of $l_{1}=28 \mathrm{~mm}$ and $l_{2}=10 \mathrm{~mm}$.

Multiple concentric rings in CR have been experimentally reported in a cascade of two [33, 75, 76], three [77] and up to four [78] BCs, with interesting applications in lasing [79], particle trapping [80], vortex generation [81] and free space optical communications [82. The diffracting wave theory of CR has been extended by Berry to the case of cylindrically symmetric beams propagating through a cascade of up to $N$ BCs [75, 83, providing an accurate description of the phenomenon in terms of Bessel functions. Additionally, the extension to non-cylindrically symmetric input beams presented in Section 2.2 .2 can be also implemented to accurately predict the pattern of input beams propagating through a cascade of BCs, as shown in Fig. 2.16 for a cascade of two biaxial crystals and a circularly polarized elliptical input beam. However, the diffractive theory 
of CR has two major drawbacks: 1) its highly demanding from a computational point of view and 2) it does not offer any intuition on the final pattern after an arbitrary large cascade of crystals.
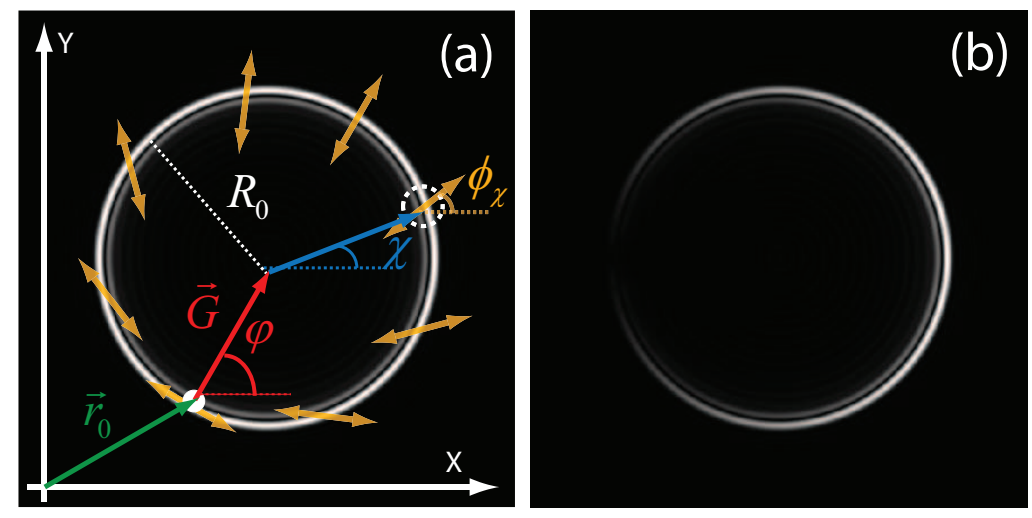

Figure 2.17: (a) CR ring at the focal plane for a circularly polarized input beam. Orange double arrows show the polarization distribution at each point of the $\mathrm{CR}$ ring. $\mathbf{G}=$ $R_{0}[\cos \varphi, \sin \varphi]$ gives the transverse displacement that the $\mathrm{CR}$ ring (with radius $R_{0}$ ) suffers with respect to the input beam (white dot), with original position $\mathbf{r}_{\mathbf{0}} . \varphi$ gives the orientation of the plane of optic axes. $\xi$ is the polar angle of a given point on the ring, with azimuth of the linear polarization $\phi_{\xi}$. (b) CR pattern for a linearly polarized input beam with azimuth of the linear polarization $\phi_{E}=30^{\circ}$.

In this Section we provide a simpler approach based in the wave-vector and polarization interpretation of CR shown in Section 2.3, Our aim is to offer a practical guideline of the final pattern for $\mathrm{CR}$ in cascaded BCs at the focal plane, including the case of linearly polarized input beams.

\subsubsection{Coordinates system and single crystal configuration}

The scheme of our experimental arrangement is shown in Fig. 2.18, A circularly polarized Gaussian light beam is focused with a lens (FL), passing along the optic axis of a cascade of up to three biaxial crystals $\left(\mathrm{BC}_{i}\right)$ rotated by angles $\varphi_{i}(i=1,2,3)$ around their aligned optic axes. Finally, an imaging lens (IL) projects the focal plane onto the CCD camera.

Single annular pattern with fine Poggendorff splitting is observed when the first crystal is placed alone. We introduce the transverse coordinates XY, see Fig. 2.17(a). The $\mathrm{BC}_{1}$ orientated at an angle $\varphi_{1}$ transforms the input Gaussian beam (with position vector $\mathbf{r}_{0}$ ) into the $\mathrm{CR}$ light ring, whose center position is given by

$$
\mathbf{r}_{1}=\mathbf{r}_{0}+\mathbf{G}_{1} \text {. }
$$

From Eq. (2.42), the position of any point of the CR ring (represented by the polar 
angle $\chi$, see Fig. $2.17(\mathrm{a})$ ), is described by

$$
\mathbf{r}_{1}(\chi)=\mathbf{r}_{0}+\mathbf{G}_{1}+\left|\mathbf{G}_{1}\right|[\cos (\chi), \sin (\chi)] .
$$

The polarization azimuth, $\Phi_{\chi}$, at each point of the ring is related to its position along the $\mathrm{CR}$ ring through

$$
\Phi_{\chi}=\frac{\chi+\varphi_{1}}{2}
$$

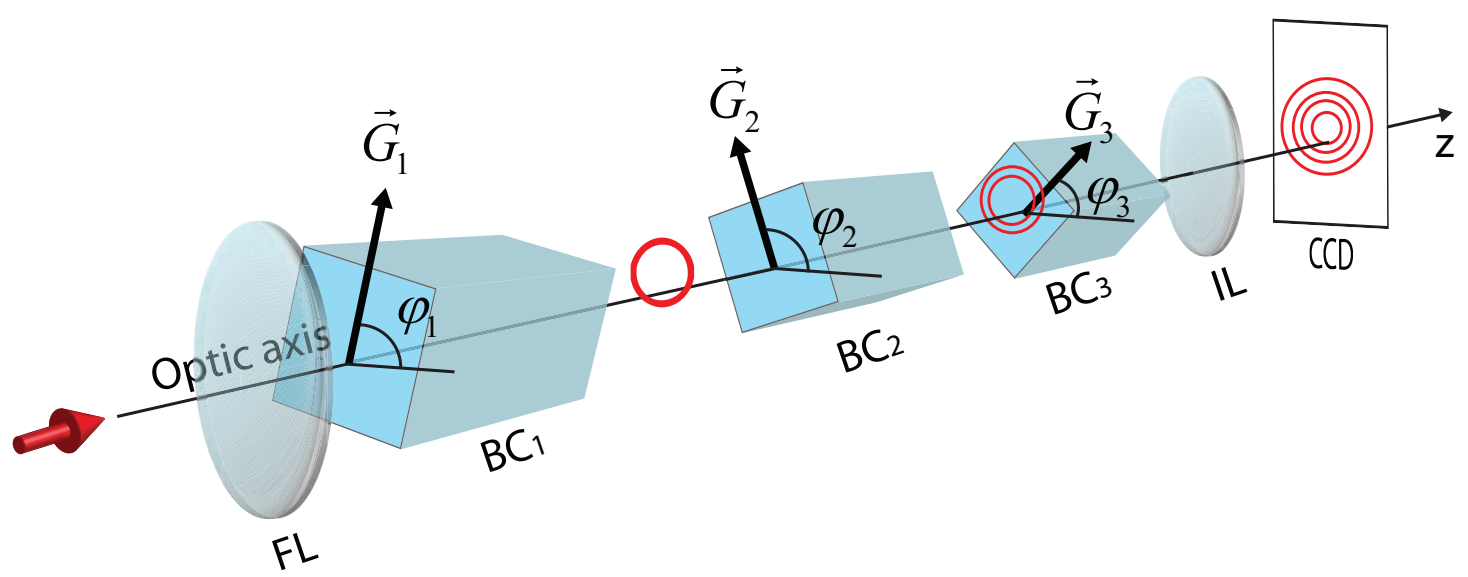

Figure 2.18: Experimental set-up. An input beam propagating along the optic axis of a cascade of biaxial crystals $\left(\mathrm{BC}_{i}\right)$ (with orientation $\varphi_{i}$ ) is focused with a lens (FL) and transformed by the $\mathrm{CR}$ phenomenon in the cascade. An imaging lens (IL) projects the final pattern into the CCD camera.

\subsubsection{Multiple crystals}

To investigate the origin of multiple rings generated by a cascade of BCs, part of the conically refracted beam is selected (filtered) by placing a pinhole at the CR ring after the first crystal, as schematically shown by the dashed white circle in Fig. 2.17(a). Every point of the ring is a CR-filtered beam defined by the polar angle $\chi$ of the filtering (which also defines the plane of wave-vectors $\Phi_{K}=\chi$ [54]) and by the plane of its electric field vector with polarization azimuth $\Phi_{\chi}$ given by Eq. (2.44). CR-filtered beams passing through a BC do not produce full ring pattern, but refract (split) into two orthogonally linearly polarized beams [73]. Their positions correspond to two diagonally opposite points of the otherwise expected CR ring for a Gaussian input beam. Their geometric center is

$$
\mathbf{r}_{2}=\mathbf{r}_{1}+\mathbf{G}_{2}=\mathbf{r}_{0}+\mathbf{G}_{1}+\mathbf{G}_{2},
$$

while their polar angles and transverse positions are defined by the filtering angle $\chi$ of the input beam

$$
\begin{array}{r}
\chi_{2}^{(1)}=\chi, \chi_{2}^{(2)}=\chi+\pi ; \\
\mathbf{r}_{2}\left(\chi_{2}^{(1,2)}\right)=\mathbf{r}_{1}(\chi)+\mathbf{G}_{2} \pm\left|\mathbf{G}_{2}\right|[\cos (\chi), \sin (\chi)]
\end{array}
$$


where the superscript distinguishes the refracted (output) beams. Their polarization azimuths $\Phi_{\chi_{2}^{(1,2)}}$ are defined by Eq. (2.44) with $\chi$ being replaced by $\chi_{2}^{(1,2)}$ given in Eq. (2.46) and $\varphi_{1}$ being replaced by $\varphi_{2}$.
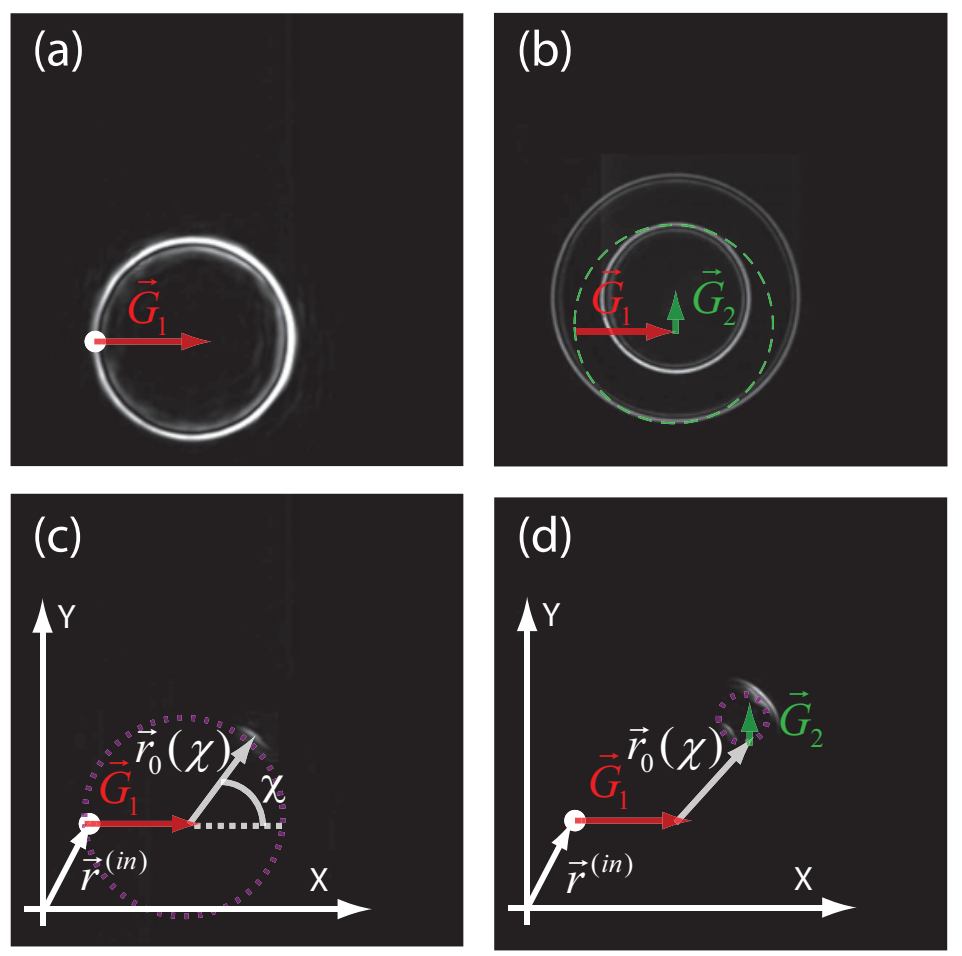

Figure 2.19: (a,b) Experimental intensity ring pattern(s) at the focal plane for a cascade formed by (a) 1 and (b) 2 biaxial crystals (BC). The dotted green ring show the CR ring that should appear in the absence of the second BCs. Red and green arrows correspond to $\mathbf{G}_{1}$ and $\mathbf{G}_{2}$ respectively. (c,d) Experimental intensity patterns obtained by using a beam filtered at $\chi=45^{\circ}$ (represented with gray vector, $\mathbf{r}_{0}(\chi)=\mathbf{r}^{(i n)}+\mathbf{G}_{1}+\frac{1}{\sqrt{2}}\left|\mathbf{G}_{1}\right|[1,1]$ ) from the $\mathrm{CR}$ ring of the first $\mathrm{BC}$. White vector gives the position of the input beam. The purple dotted ring show the CR ring that would appear if the CR-filtered beam was a Gaussian beam.

By combining Eq. (2.43) with Eqs. (2.45) and (2.47), the latter reads

$$
\mathbf{r}_{2}\left(\chi_{2}^{(1,2)}\right)=\mathbf{r}_{2} \pm R_{2}^{(1,2)}[\cos (\chi), \sin (\chi)]
$$

where $R_{2}^{(1,2)}=|| \mathbf{G}_{\mathbf{1}}| \pm| \mathbf{G}_{\mathbf{2}} \|$. To relate the angle between consecutive crystals, we define $\varphi_{n m} \equiv \varphi_{n}-\varphi_{m}$. The intensity splitting distribution between the refracted beams (derived from Section 2.3) is

$$
\begin{aligned}
& I_{2}^{(1)}=I_{\chi} \cos ^{2}\left(\Phi_{\chi_{2}^{(1)}}-\Phi_{\chi}\right)=I_{\chi} \cos ^{2}\left(\frac{\varphi_{21}}{2}\right), \\
& I_{2}^{(2)}=I_{\chi} \cos ^{2}\left(\Phi_{\chi_{2}^{(2)}}-\Phi_{\chi}\right)=I_{\chi} \sin ^{2}\left(\frac{\varphi_{21}}{2}\right),
\end{aligned}
$$


where $I_{\chi}=I_{\chi, C P}=\frac{P_{0}}{A}$ for a circularly polarized input beam, while $I_{\chi}=I_{\chi, L P}=$ $\frac{2 P_{0}}{A} \cos ^{2}\left(\Phi_{\chi}-\Phi_{0}\right)=2 I_{\chi, C P} \cos ^{2}\left(\frac{\chi+\varphi_{1}}{2}-\Phi_{0}\right)$ for the linearly polarized case. $P_{0}$ is the input beam's power, which redistributes over the area $A=4 \pi w_{0} R$ occupied by the CR ring. Note that if $R=0$, then $A=\pi w_{0}^{2}$, where $w_{0}$ is the waist radius of the focused input beam. The two refracted beams after the $\mathrm{BC}_{2}$ are also CR-filtered beams, that are described by their transverse positions $\mathbf{r}_{2}^{(1,2)}=\mathbf{r}\left(\chi_{2}^{(1,2)}\right)$ and by the set of parameters $\left[\chi_{2}^{(1,2)}, \Phi_{\left.\chi_{2}^{(1,2)}\right]}\right.$.

Experimental results on CR-filtered beams in a cascade of two crystals are presented in Fig. 2.19, As it can be appreciated, a CR-filtered beam from the CR ring generated by the first crystal refracts as two beams after passing through the second crystal. When the ring of the first crystal is not filtered, the transverse intensity pattern obtained after the second crystal forms a pair of concentric CR rings each of which with Poggendorff splitting.

Multiple rings formation is obtained by considering the full range $\chi \in[0,2 \pi)$. In this case, Eqs. (2.48), (2.49) and (2.50) define two concentric rings with common center at $\mathbf{r}_{2}$, radii $R_{2}^{(i)}$ and azimuthal intensities $I_{2}^{(i)}$ :

$$
\begin{array}{r}
\mathbf{r}_{2}=\mathbf{r}_{0}+\mathbf{G}_{1}+\mathbf{G}_{2}, \\
R_{2}^{(i)}=|| \mathbf{G}_{\mathbf{1}}| \pm| \mathbf{G}_{\mathbf{2}} \|(i=1,2), \\
I_{2, C P}^{(1)}=\frac{P_{0}}{A_{2}^{(1)}} \cos ^{2}\left(\frac{\varphi_{21}}{2}\right), \\
I_{2, C P}^{(2)}=\frac{P_{0}}{A_{2}^{(2)}} \sin ^{2}\left(\frac{\varphi_{21}}{2}\right),
\end{array}
$$

being $A_{2}^{(i)}=4 \pi w_{0} R_{2}^{(i)}$ if $R_{2}^{(i)} \neq 0$ and $A_{2}^{(i)}=\pi w_{0}^{2}$ if $R_{2}^{(i)}=0$. In other words, the second crystal shifts the center of the ring pattern and splits the parental CR ring into two concentric ones, as observed experimentally for the cascade of two crystals, see Fig. 2.20(a1). For input beams with linear polarization, the azimuthal intensity of the light ring patterns are:

$$
\begin{array}{r}
I_{2, L P}^{(1)}=2 I_{2, C P}^{(1)} \cos ^{2}\left(\frac{\chi+\varphi_{1}}{2}-\Phi_{0}\right), \\
I_{2, L P}^{(2)}=2 I_{2, C P}^{(2)} \cos ^{2}\left(\frac{\chi+\varphi_{1}}{2}-\Phi_{0}+\frac{\pi}{2}\right),
\end{array}
$$

for $\left|\mathbf{G}_{\mathbf{2}}\right|>R_{1}$ and

$$
\begin{aligned}
& I_{2, L P}^{(1)}=2 I_{2, C P}^{(1)} \cos ^{2}\left(\frac{\chi+\varphi_{1}}{2}-\Phi_{0}\right), \\
& I_{2, L P}^{(2)}=2 I_{2, C P}^{(2)} \cos ^{2}\left(\frac{\chi+\varphi_{1}}{2}-\Phi_{0}\right),
\end{aligned}
$$

for $\left|\mathbf{G}_{\mathbf{2}}\right|<R_{1}$. Experimental patterns and corresponding theoretical simulations of a cascade of two biaxial crystals for input beams with linear and circular polarizations are shown in box (1) of Fig. 2.20. 
The third biaxial crystal $\left(\mathrm{BC}_{3}\right)$, once added into the cascade, splits each CR-filtered beam into two CR filtered beams with parameters defined by Eqs. (2.46), (2.47), (2.49) and (2.50) as previously described. Therefore, four CR filtered beams appear at the positions defined by Eq. (2.48) with parameters $\mathbf{r}_{3}=\mathbf{r}_{0}+\mathbf{G}_{1}+\mathbf{G}_{2}+\mathbf{G}_{3}$ and $R_{3}^{(i)}=$ $\left|R_{2}^{(1,2)} \pm\right| \mathbf{G}_{\mathbf{3}}||(i=1,2,3,4)$. For the full range $\chi \in[0,2 \pi)$ the latter results predict four concentric rings as shown in Fig. 2.20(a2), centered at $\mathbf{r}_{3}$ and with radii $R_{i}^{(3)}$ and corresponding azimuthal intensities $I_{3}^{(i)}$ as follows

$$
\begin{array}{r}
\mathbf{r}_{3}=\mathbf{r}_{0}+\mathbf{G}_{1}+\mathbf{G}_{2}+\mathbf{G}_{3}, \\
R_{3}^{(i)}=|| \mathbf{G}_{\mathbf{1}}| \pm| \mathbf{G}_{\mathbf{2}}|| \pm\left|\mathbf{G}_{\mathbf{3}}\right| \mid(i=1,2,3,4), \\
I_{3}^{(1)}=\frac{P_{0}}{A_{3}^{(1)}} \cos ^{2}\left(\frac{\varphi_{21}}{2}\right) \cos ^{2}\left(\frac{\varphi_{21}}{2}\right), \\
I_{3}^{(2)}=\frac{P_{0}}{A_{3}^{(2)}} \sin ^{2}\left(\frac{\varphi_{32}}{2}\right) \cos ^{2}\left(\frac{\varphi_{21}}{2}\right), \\
I_{3}^{(3)}=\frac{P_{0}}{A_{3}^{(3)}} \cos ^{2}\left(\frac{\varphi_{32}}{2}\right) \sin ^{2}\left(\frac{\varphi_{21}}{2}\right), \\
I_{3}^{(4)}=\frac{P_{0}}{A_{3}^{(4)}} \sin ^{2}\left(\frac{\varphi_{32}}{2}\right) \sin ^{2}\left(\frac{\varphi_{21}}{2}\right),
\end{array}
$$

for CP input beams. For LP input beams, corresponding expressions can be obtained using Eqs. (2.55) -(2.58) and taking into account whether $\left|\mathbf{G}_{3}\right|<R_{2}^{(i)}$ or $\left|\mathbf{G}_{3}\right|>R_{2}^{(i)}$ $(i=1,2)$. Explicit formulation is not presented here since for a cascade of $N=3$ BCs there are $3 !=6$ possible combinations $\times 4$ light rings $=24$ formulae. Experimental patterns and corresponding theoretical simulations for a cascade of three biaxial crystals for linearly and circularly polarized input beams are presented in box (2) of Fig. 2.20.

Patterns for a cascade of $N$ biaxial crystals with characteristic vectors $\mathbf{G}_{i}$ can be obtained by applying Eq. (2.59) (position of the center) Eq. (2.60) (radii of each ring) and Eqs. (2.61)-(2.64) (intensity of each ring) recursively. In this case, up to $2^{N-1}$ concentric rings appear at the focal plane, as it was recently shown if Refs. [75, 78, 83] for circularly polarized input beams.

\subsection{Conclusions}

In this chapter we have shown the fundamental features of the CR phenomenon under three different perspectives. In the first part of the chapter, the diffractive solution of CR has been presented. We have demonstrated that this formulation allows predicting the free space evolution of light beams propagated through a biaxial crystal along any direction, including one of the optic axes. For a cylindrically symmetric and uniformly polarized beam propagating along one of the optic axes, simplified equations and numerical calculations for the particular case of a Gaussian input beam have been presented. In the general case, we have reported both theoretically and experimentally 
the transition from double refraction to CR of a Gaussian and an elliptical input beam propagating within a biaxial crystal. Note that this formalism is also suitable to predict the transformation of non-homogeneously polarized beams after propagating through a biaxial crystal, as we have demonstrated for a radially and azimuthally polarized input light beams [53].

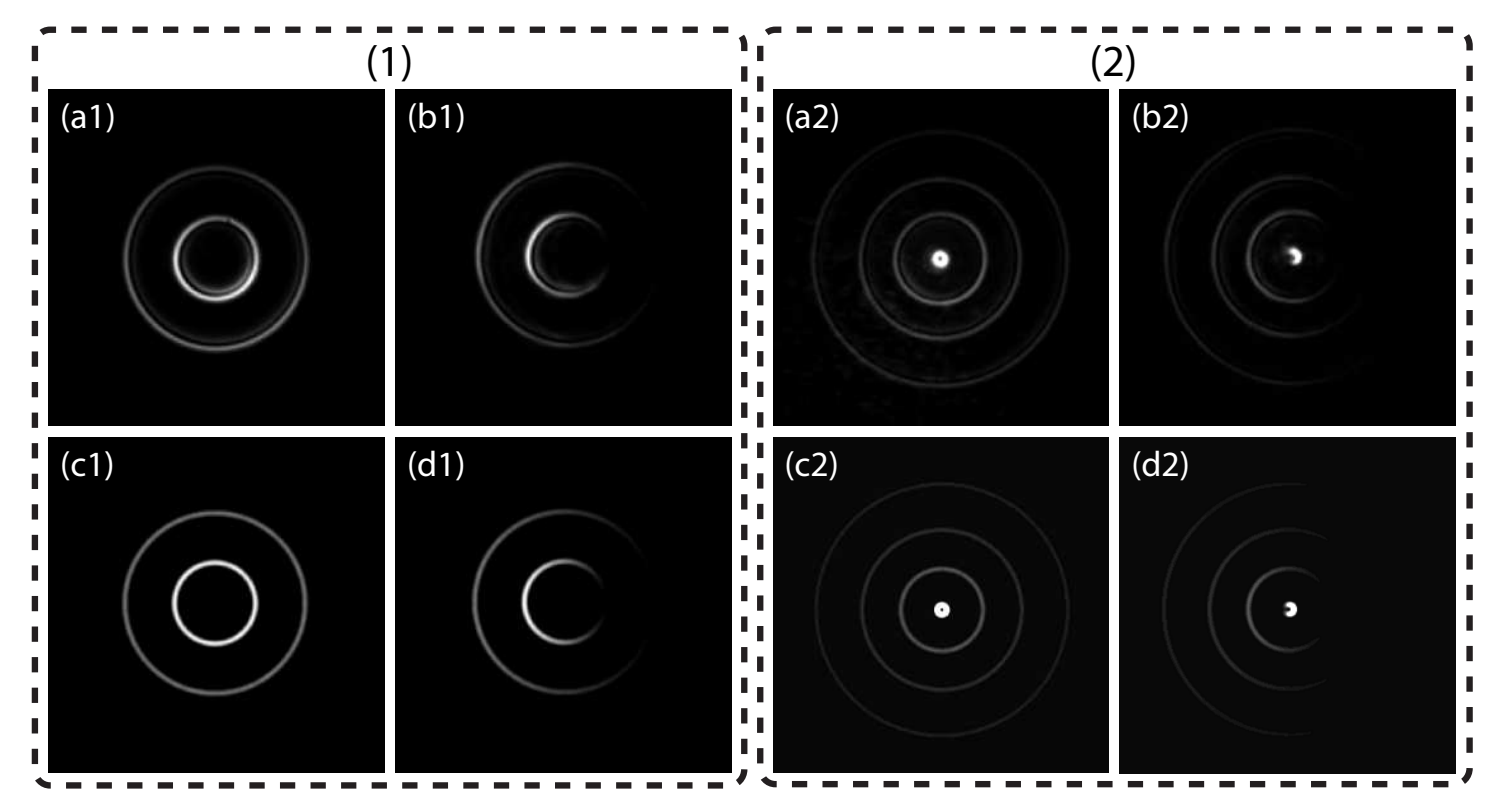

Figure 2.20: Transverse intensity patterns for a cascade of 2 BCs (box 1) and 3 BCs (box 2). Figures (ai,bi) are experimental data and (ci,di) $(i=1,2)$ the corresponding theoretical simulations. First column corresponds to a circularly polarized input beam, while second column corresponds to a linearly polarized input beam with azimuth $\Phi_{0}=$ $0^{\circ}$. Experimental parameters of the BCs: $L_{1}=27.31 \mathrm{~mm}, \varphi_{1}=0^{\circ} ; L_{2}=10.66 \mathrm{~mm}$, $\varphi_{2}=90^{\circ} ; L_{3}=18.29 \mathrm{~mm}, \varphi_{3}=180^{\circ}$. The focal length of the focusing lens (FL, see Fig. 2.18) is $200 \mathrm{~mm}$.

Then, we have presented the wave-vector and polarization dependence of the internal conical refraction phenomenon in biaxial crystals. We have experimentally proved that this phenomenon is only one particular case of refraction associated to beams possessing continuous axial cylindrical symmetry. In fact, we have reported that strongly elliptical beams do not generate the complete characteristic light ring of $\mathrm{CR}$ when they propagate along one of the optic axis of a biaxial crystal but split, in general, into two beams. We have derived expressions for the positions and relative intensities of the resulting refracted beams. These expressions play an analogous role as the Malus law but for biaxial crystals and can be used to predict the transverse intensity pattern of much more involved incident beams, as it has been demonstrated here for an input axicon beam.

In the third Section of the chapter, we have demonstrated the dual-cone nature of 
the CR phenomenon by using an azimuthally segmented polarizer that mimics the CR polarization distribution to generate a non-homogeneously polarized beam. We have proved that such device allows for selecting between the two CR cones and shown the experimental free-space conical beam evolution for a Gaussian input beam. A mirrorsymmetric beam evolution with respect to the focal plane for the two light cones has been obtained. In addition, we have demonstrated that the $\mathrm{CR}$ cones $\mathbf{C}_{+}$and $\mathbf{C}_{-}$have their vertexes at the Raman spot behind and before the focal plane, respectively. It has been also reported the generation of two bright rings split by the Poggendorff dark ring at the focal plane, which can be understood in terms of the interference produced by the difference on the divergence of the two co-propagating light cones.

Finally, it has been presented an extension to a cascade of biaxial crystals, that leads to a transverse intensity pattern formed by multiple concentric rings. We have demonstrated that such pattern can be described by using the wave-vector and polarization formalism previously commented and we have shown that a cascade formed by $N$ BCs generates up to $2^{N-1}$ rings. A Simple formulation that allows deducing the position, radii and intensity for each light ring has been also presented. Finally, we have reported the transverse intensity pattern of cascaded CR from a linearly polarized input beam, showing that the final pattern depends on the relative position of the BCs in the cascade, i.e., it is a non-commutable operation. 

CHAPTER 3

Beam shaping with conical refraction

This Chapter demonstrates the influence of the $\rho_{0} \equiv R_{0} / w_{0}$ parameter over the CR beam evolution. While for $\rho_{0} \gg 1$ the characteristic pair of concentric bright rings with Poggendorff splitting are generated, for $\rho_{0} \approx 1$ the CR beam changes radically. In Section 3.1 we discuss all the previous works that related with CR for low values of $\rho_{0}$. Section 3.1 is devoted to show CR beams that can be obtained for different values of $\rho_{0}<10$. Our investigations on the generation of a three-dimensional (3D) dark focus with CR are presented in Section 3.2. We demonstrate that in this case the CR beam forms a perfect optical bottle, i. e. a null-intensity region in space surrounded for regions of higher intensity in all directions. In Section 3.3 we present a novel SuperGaussian beam obtained with CR. Super-Gaussian beams are beams whose intensity distribution is flat at the top and that decays smoothly at the edges. We discuss the beam propagation of the super-Gaussian beam and show that it has a confocal parameter three times larger than the one that would be obtained from a Gaussian beam. The main advantages CR for beam shaping and conclusions of the Chapter are discussed in Section 3.4

The research contained in this chapter has been has been done in collaboration with Edik U. Rafailov from the Aston University, and Hiromitsu Tomizawa from the Japan Synchrotron Radiation Research Institute and has been published in Refs. 66, 68,

\subsection{Introduction}

The evolution of the CR beam depends strongly on the $\rho_{0}=R_{0} / w_{0}$ parameter. Up to now, we have centered our attention to the condition $\rho_{0} \gg 1$, which covers most of the works on the field. However, there have been some investigations on the CR 
beam dependence with the $\rho_{0}$ parameter. The first contribution that can be found in this sense was from Belsky and Stepanov [29], although they only reported a graph with the $1 \mathrm{D}$ radial intensity distribution at the focal plane for different values of $\rho_{0}$. A similar analysis was made by Belafhal [31. Hellström and co-workers, designed a Yb:KGW laser based on CR and reported transverse intensity patterns obtained with low values of $\rho_{0}$, although no detailed investigation on this issue can be found [84. The first real experimental attempt to take profit of the $\rho_{0}$ parameter was carried out by Peet, who proposed the use of the biaxial crystals as a versatile mode converter between Heremite-Gauss and Laguerre-Gauss beams throughout the CR phenomenon [76]. The same author, analyzed in detail the $B_{0}$ and $B_{1}$ components of the CR beam to improve the directivity of the input beam [63] and also to generate vortex-like structures in the far field of the $\mathrm{CR}$ beam [65]. Rosen and co-workers proposed a sub wavelength localization scheme for optical imaging based in $\mathrm{CR}$ under the condition $\rho_{0} \approx 1$ [85].

Although being very interesting, none of the previously commented works studied in detail the beam evolution of the obtained CR beams for low values of $\rho_{0}$. In this chapter, we do so for two very particular values of $\rho_{0}$ leading to a $3 \mathrm{D}$ dark focus $\left(\rho_{0}=0.924\right)[66$ ] and a Super-Gaussian beam $\left(\rho_{0}=0.445\right)$ 68]. Before discussing in detail the features of the 3D dark focus and the Super-Gaussian beam, in this section we show how the $\rho_{0}$ parameter affects the intensity pattern of the CR beam. In Fig. 3.1(a) we show the radial intensity distribution variation with $\rho_{0}$ obtained from a circularly polarized Gaussian input beam and using Eqs. (2.11) -(2.16). For $\rho_{0} \geq 3$ the characteristic two bright rings with the Poggendorff splitting can be observed. In contrast, for lower values of $\rho_{0}$, the two bright rings and, in particular, the inner bright ring are not clearly distinguished. This can be better visualized in Figs. 3.1(b)-(i), where transverse intensity patterns in the $X Y$ plane at $Z=0$ for different values of $\rho_{0}$ are presented. As $\rho_{0}$ decreases, the transverse intensity pattern differs from the characteristic $\mathrm{CR}$ bright rings. At $\rho_{0} \approx 1.5$ the inner ring collapses into a central spot, at $\rho_{0} \approx 1$ only a single (doughnut-like) ring is found and for $\rho_{0}<1$ the ring becomes imperceptible. Since for $\rho_{0}<1$ the transverse intensity pattern evolves from a doughnut-like ring to a Gaussian-like beam, it seems that it is possible to find a value of $\rho_{0}$ where the radial intensity distribution is flat at its top.

\subsection{Generating a 3D dark focus with conical refraction}

Optical beams with dark regions of exactly zero intensity are rare objects usually related to optical singularities. Their applications range from particle trapping [66, subdiffraction limited tighter focusing [87] and plasmon excitation [88] to laser machining [89. Laguerre-Gauss beams are well known examples of light beams possessing optical vortices forming a straight nodal line surrounded by light [90]. More involved structures of closed loop nodal lines, their threading, knotting and linking have been demonstrated recently [91 94]. 


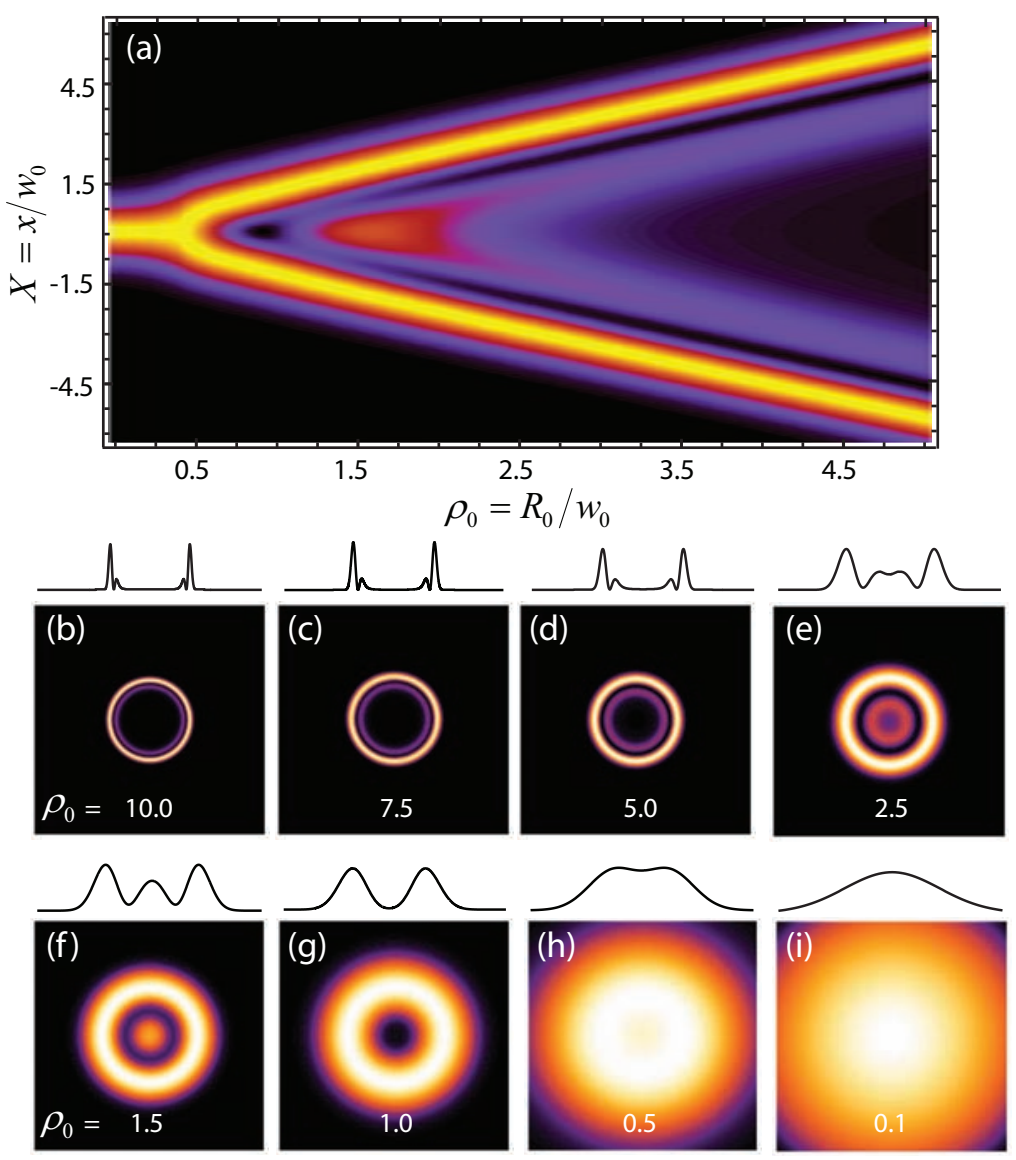

Figure 3.1: (a) 2D density plot of the radial intensity distribution at $I\left(\rho, \rho_{0}, Z=0\right)$, as given by Eq. (2.12)-(2.17). (b)-(i) Transverse intensity distributions at the focal plane $I_{\mathrm{CP}}(\rho, Z=0)$ for an input Gaussian beam of circular polarization as given by Eq. (2.12) -(2.17) for different values of the control parameter $\rho_{0}$.

Bottle beams [95] are another example of optical beams with a dark region, more precisely they are also named optical dark potentials. Ideally, bottle beams comprise zero electric amplitude at one spatial point surrounded in all directions by regions with relatively high intensity. In practice, the intensity minimum in bottle beams is not exactly equal to zero because of different experimental imperfections. Various methods and techniques have been proposed to produce 3D optical dark potentials in a controllable way, such as creating an intensity minimum by surrounding a region in 3D space with several beams [96, 97, crossing at least two cylindrical-vector and vortex beams with a phase dislocation along the beam axis that leads to a zero-intensity point [98], by destructive interference of several Laguerre-Gauss light beams [95,99] or using uniaxial c-cut crystals [100]. However, these methods have several drawbacks such as the fact that in some of them the intensity minimum is not exactly equal to zero, the extreme precise control on the optical elements being used, the field fluctuations introduced at and close to the zero-amplitude point, or the non trivial generation of Laguerre-Gauss 
and cylindrical-vector and vortex beams [101]. Optical bottle beams have applications in optical tweezers for trapping particles with a refractive index lower than the surrounding medium, using the photophoretic force for instance [86], or in cold atom trapping with the possibility of creating an all optical blue-detuned trap that operates in the zero-intensity region [102].

In this section, we present a robust, easy, and compact alternative to the previously cited methods to generate an optical bottle beam by transforming a fundamental monochromatic Gaussian beam into a beam with a 3D dark focus, i.e. a beam with a point of exact null intensity surrounded in all directions by regions of higher intensity, by means of $\mathrm{CR}$.

\subsubsection{Axial intensity for $\rho_{0} \approx 1$}

Let us consider a focused monochromatic input beam propagating along one of the optic axis of a biaxial crystal. In what follows, we will assume a fundamental Gaussian input beam whose Rayleigh length and waist radius are given by $z_{R}$ and $w_{0}$, respectively. If focusing lenses are used, the beam waist position is located at the focal plane of the lens. Since $R_{0}=l \alpha$ is characterized by the length $(l)$ and conicity parameter $(\alpha)$ of the crystal, the $\rho_{0}$ parameter can be easily controlled experimentally by modifying $w_{0}$, e. g. by changing the focal length of the lens used in the experiments.

For a circularly polarized beam, at the beam center $(\rho=0)$, from Eq. (2.14), the on $Z$-axis intensity distribution is defined solely by the function $B_{0}(\rho=0, Z)$ since $J_{1}(0)=0$ and, therefore, $B_{1}(\rho=0, Z)=0$, see Eq. (2.13). In other words,

$$
I_{\mathrm{CP}}(\rho=0, Z)=\left|\frac{1}{(2 \pi)} \int_{0}^{\infty} a(k) e^{-i \frac{1}{2 n} k^{2} Z^{2}} \cos \left(k \rho_{0}\right) d \phi_{k}\right|^{2}
$$

The former can be evaluated analytically for a Gaussian input beam with Fourier transform defined by Eq. (2.17), yielding:

$$
B_{C}(0, Z)=\frac{1}{w_{Z}} \frac{d}{d X} \mathrm{~F}(X)=\frac{1}{w_{Z}}\left[1+i \sqrt{\pi} X e^{-X^{2}} \operatorname{erf}(i X)\right]
$$

where $X=\rho_{0} / \sqrt{w_{Z}}$ and $w_{Z}=1+i Z . \mathrm{F}(X)$ and $\operatorname{erf}(X)$ denote Dawson and error functions, respectively. Dawson function satisfies the equation $d \mathrm{~F}(X) / d X=1-2 X \mathrm{~F}(X)$. 


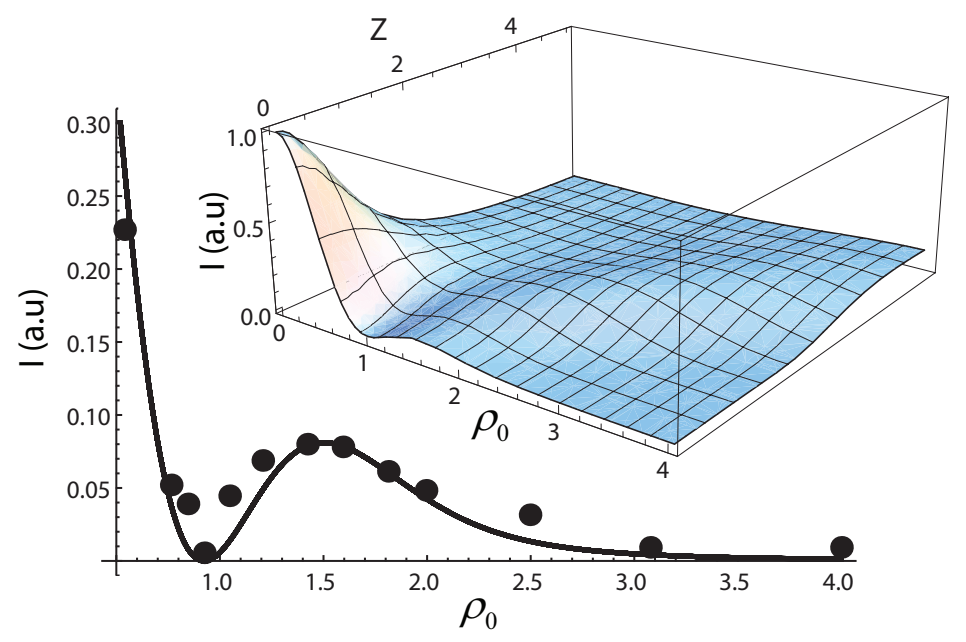

Figure 3.2: On-axis intensity at the focal plane center, $I\left(\rho=0, \rho_{0}, Z=0\right)$ as a function of the control parameter $\rho_{0}=R_{0} / w_{0}$. Black solid circles represent the experimental data obtained when a focused Gaussian beam propagates along the optic axis of a biaxial crystal. The inset shows the on-axis intensity $I\left(\rho=0, \rho_{0}, Z\right)$.

The evolution of the normalized intensity at the beam center for different values of $\rho_{0}$ is shown in Fig. 3.2. At $Z=0$ (1D plot), for $\rho_{0} \geq 3$ the intensity at the beam center is close to 0 . From $\rho_{0} \geq 3$ down, the axial intensity increases, having a relative maximum at $\rho_{0} \approx 1.5$. Then, the intensity decreases newly and a minimum is found at $\rho_{0} \approx 1$. From this value of $\rho_{0}$ down, the axial intensity increases until a maximum at $\rho_{0}=0$, where no $\mathrm{CR}$ can be observed. The surface plot reproduces the $1 \mathrm{D}$ plot at different axial positions. As commented in the previous Chapter, for large values of $\rho_{0}$, an axial intensity maximum (Raman spot) is found far from the focal plane $(Z=0)$. For low values of $\rho_{0}$ the absolute axial maximum is shifted towards $Z=0$. However, for $\rho_{0} \approx 1$ the absolute axial intensity maximum is found again far from the focal plane.

From Fig. 3.2, it is clear that Eq. (3.2) has a solution with zero amplitude at the beam center $(\rho=0, Z=0)$ that corresponds to the maximum of the Dawson function $\mathrm{F}(X)$. Thus, by numerically solving $B_{C}(0, Z)=0$ from Eq. (3.2), one expects to obtain a dark focus (DF) (zero amplitude) for

$$
\rho_{0}^{\mathrm{DF}}=0.924
$$

where the superscript 'DF' means dark focus. 


\subsubsection{Characteristics of the 3D dark focus beam}
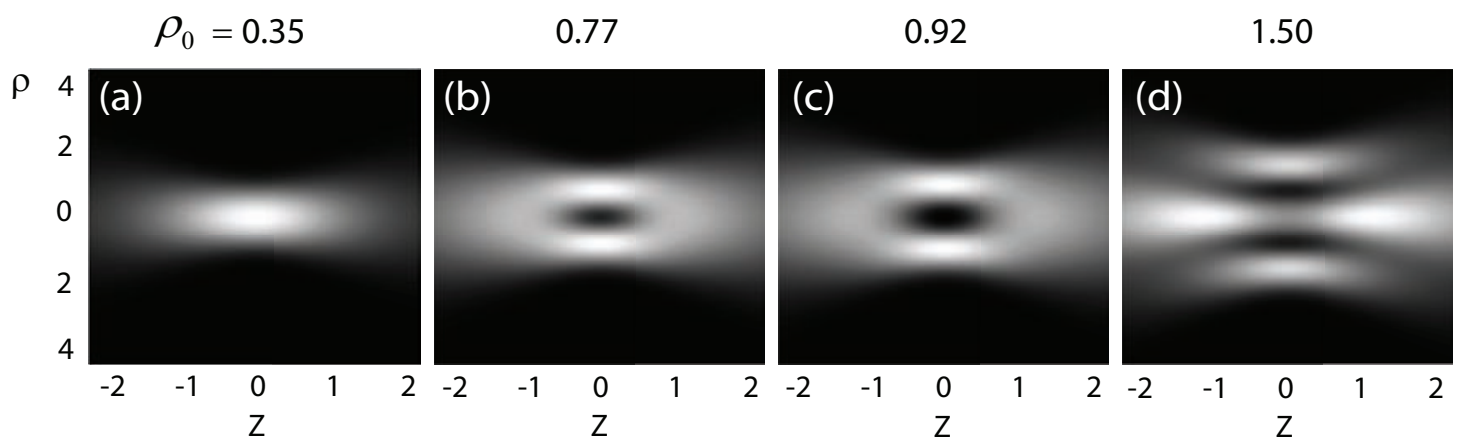

Figure 3.3: Slice of the intensity distributions $I_{\mathrm{CP}}(\rho, Z)$ along the propagation direction for an input Gaussian beam of circular polarization as given by Eqs.(2.12)-(2.14) for different values of the control parameter $\rho_{0}$.

Since the $Z$-axis is the symmetry axis of the output beam, the $3 \mathrm{D}$ distribution of the electric field can be represented by a two dimensional slice of intensity $I_{\rho Z}=I(\rho, Z)$ as shown in Fig. 3.3. For $\rho_{0}<0.5$, a single bright spot is observed whose maximum intensity corresponds to the center of the focal plane, see Fig. 3.3(a). For larger values of $\rho_{0}$, this maximum is laterally shifted in the focal plane and an intensity minimum appears at $\rho=Z=0$, see Figs. 3.3(b,c). In particular, the 3D dark focus or perfect bottle beam found for $\rho_{0}=\rho_{0}^{\mathrm{DF}}=0.924$ [Fig. 3.3(c)] disappears for larger values of $\rho_{0}$, see Fig. 3.3(d). As it is seen in Fig. 3.3(c), the 3D dark focus with a zero intensity point at the beam center is surrounded by a region of higher intensity in all directions. The maximum of the intensity barrier is achieved at the focal plane on a ring with radius $\rho_{\max } \approx 1.1$ and $I\left(\rho_{\max }, Z=0\right) \approx 0.2$, i.e. the peak intensity at this ring is approximately $20 \%$ of that provided by the same focused Gaussian beam without the crystal. Along the beam propagation direction, the axial intensity has maxima at $Z_{\max } \approx \pm 1.388$ with $I\left(\rho=0, Z_{\max }\right) \approx 0.14$. The minimum in the intensity barrier has a form of a ring with radius $\rho_{\theta} \approx 0.62$ that appears at a distance $Z_{\theta} \approx \pm 1.1$ with $\left|\rho_{\theta} / Z_{\theta}\right| \approx \tan 30^{\circ}$ and $I\left(\rho_{\theta}, Z_{\theta}\right) \approx 0.13$. Dark focus, see Fig $3.4(\mathrm{c})$, and dark ring, see Fig $3.4(\mathrm{~d})$, appear because of destructive interference of two cones provided by the CR phenomenon inside biaxial crystal and displaced with respect to each other along propagation direction as detailed in Chapter 2

To experimentally confirm the theoretical prediction on the possibility to generate a CR bottle beam with a 3D dark focus, we have performed experiments on CR in a plate of $\mathrm{KGd}\left(\mathrm{WO}_{4}\right)_{2}$ biaxial crystal $2.3 \mathrm{~mm}$ long yielding a $\mathrm{CR}$ ring radius of $R_{0}=39.1 \mu \mathrm{m}$. The Gaussian input beam (with waist radius of $1.5 \mathrm{~mm}$ ) obtained from a $640 \mathrm{~nm}$ diode laser coupled to a monomode fiber, as shown in Fig. 2.13. The input collimated beam is focused by a lens and the transverse intensity patterns behind the crystal are recorded at different positions along the beam propagation direction within few Rayleigh lengths. 
By varying the focusing distance of the lens, we are able to adjust the radius of the focused beam, i.e. the parameter $\rho_{0}=R_{0} / w_{0}$ in the range $\rho_{0} \in[0.3,4.0]$.

$\rho_{0}=0.55$
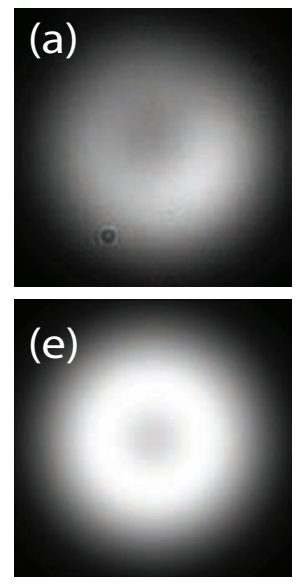

0.93
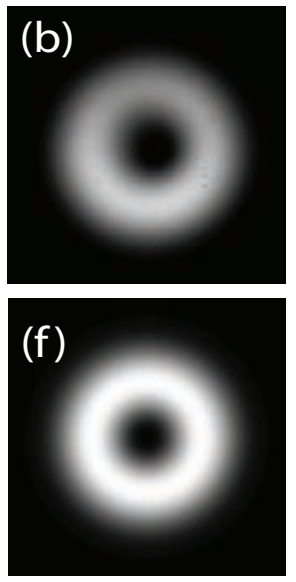

1.50
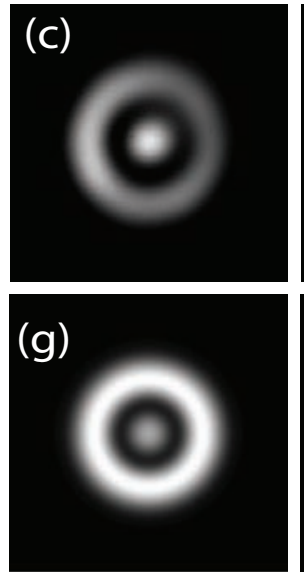

3.00
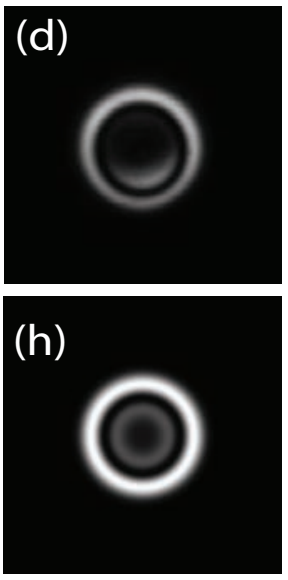

Figure 3.4: Transverse intensity patterns at the focal plane experimentally obtained (a)-(d) and theoretically calculated (e)-(h) for a circularly polarized input beam of fundamental Gaussian profile with different values of the waist radius $w_{0}$, which gives different values of control parameter $\rho_{0}$.

Normalized intensities at the center of the focal plane experimentally measured are plotted in Fig. 3.2 with black solid points, while the experimentally captured full transverse intensity patterns are represented in the upper row of Fig. 3.4 for different values of $\rho_{0}$. As indicated before, for $\rho_{0}<0.5$, the intensity pattern resembles that of a Gaussian beam. From $\rho_{0}=0.45$ to $\rho_{0}=0.92$, the intensity at the center decreases being exactly zero at $\rho_{0}=0.92$, see Figs. 3.4(a) and (b). From $\rho_{0}=0.92$ to $\rho_{0}=1.50$, it increases with a relative maximum at $\rho_{0}=1.50$, see Fig. 3.4(c). From this value on, the intensity at the center monotonically decreases and the full transverse intensity pattern becomes the standard pattern of CR with two bright rings, see Fig. 3.4(d). The waist of the focused beam has been measured by removing the crystal. For the results shown in Fig. 3.4(b), it provides the ratio $\rho_{0}^{(\exp )}=0.93$, which is close to the theoretical value $\rho_{0}^{\mathrm{DF}}$, see Eq. (3.3). The lower row in Fig. 3.4 shows the full transverse intensity patterns theoretically calculated from Eqs. (2.12)-(2.17) being in excellent agreement with the experimental results.

Figs. 3.5(a)-(d) present the experimentally measured spatial evolution of the transverse intensity patterns for $\rho_{0}^{(\exp )}=0.93 \sim \rho_{0}^{\mathrm{DF}}$ that corresponds to the case where a $3 \mathrm{D}$ dark focus appears at the focal plane. The dark focus pattern from Fig. 3.5(a) evolves to a pattern with a maximum intensity at its center as shown in Fig. 3.5(d). Clearly, a region of null intensity is surrounded by regions of higher intensity. The Rayleigh length in this case is $z_{R} \approx 8.9 \mathrm{~mm}$, which gives an estimation of the depth of the dark potential. Figs. 3.5(e)-(h) are the corresponding theoretical predictions obtained from 
Eqs. (2.12)-(2.14). Both theory and experiments are in good agreement.

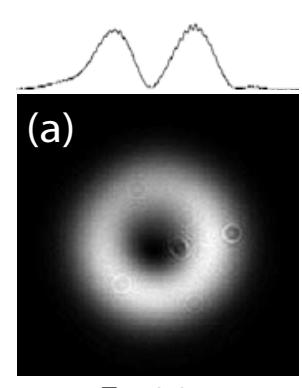

$Z=0.0$

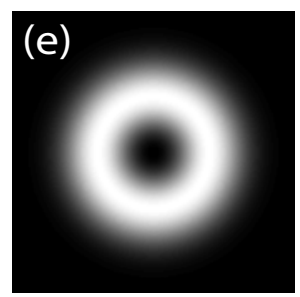

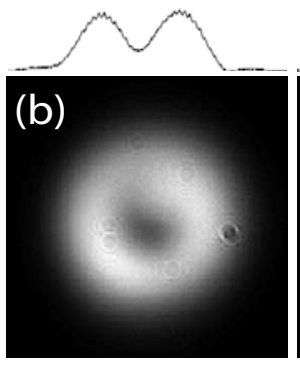

0.5

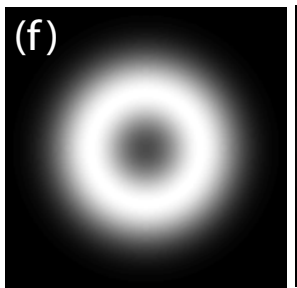

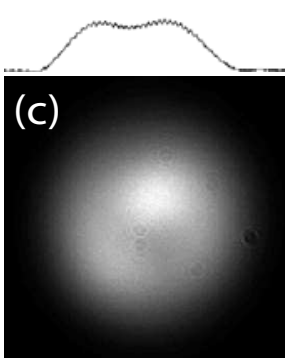

1.0

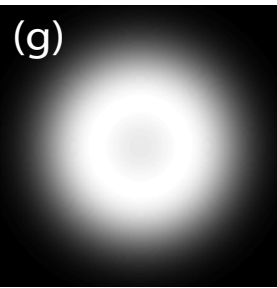

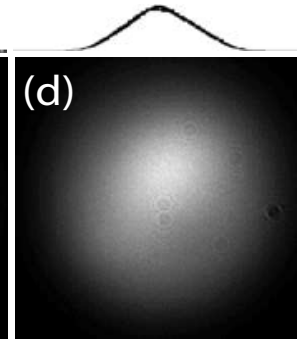

2.0

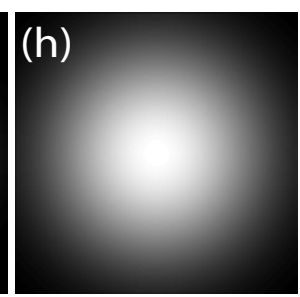

Figure 3.5: Spatial evolution of the transverse intensity patterns along the propagation distance $Z$ for $\rho_{0}^{(\exp )}=0.93 \sim \rho_{0}^{\mathrm{DF}}$, see Eq.(3.3), as experimentally obtained (a)-(d) and theoretically calculated (e)-(h), see Eqs.(2.12)-(2.14). Top insets represent the crosssection of the measured intensity distribution along the horizontal axis at the beam center.

\subsection{A Super-Gaussian conical refraction beam}

Ideally, a flat-top beam [101, 103 116] is a light beam possessing an intensity transverse profile mostly flat in the central part and sharply decaying at its edges, at variance with the Gaussian profile of the fundamental $\mathrm{TEM}_{00}$ mode. Flat-top beams are useful in a wide variety of laser applications where one needs a uniform intensity over a fixed area, such as in optical processing [101, 117, laser-driven acceleration of particles [118, 119], optical trapping [120] or gravitational-waves detectors [121. Nevertheless, the generation of flat-top beams is a non trivial task and usually diffractive optical elements are required, which suffers from several drawbacks such as losses due to inefficient mode projection or diffraction, their extreme precise control or their limited spectral range [101]. Beams whose transverse intensity profile possess sharp edges and extremely flat crosssection are ideal realizations of flat-top beams. In experimental situations flat-top beams are well approximated by super-Gaussian beams [122,123]. A super-Gaussian beam also possesses a flat intensity profile but it decays smoothly at its edges, similarly to a Gaussian beam [123]. The aim of this section is to show that a beam of super-Gaussian profile can be generated by transforming an input Gaussian beam with a biaxial crystal throughout the CR phenomenon. 


\subsubsection{Characteristics of the Super-Gaussian conical refraction beam}
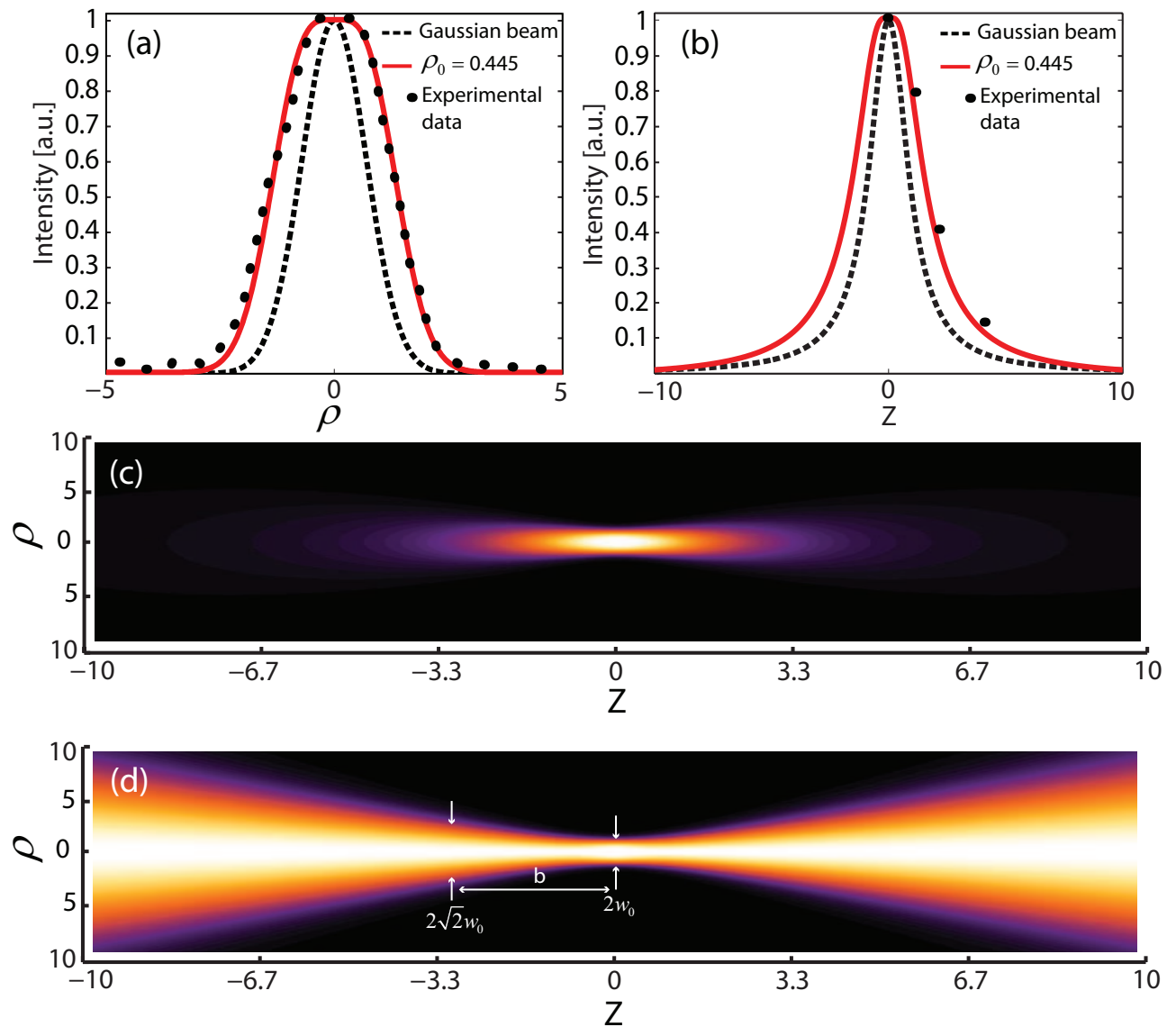

Figure 3.6: Cross-section of the intensity distribution along (a) the radial direction and (b) the beam propagation direction at the beam center for the original Gaussian beam (black dashed line) and the super-Gaussian conical refraction (SGCR) beam obtained by adjusting $\rho_{0}=0.445$ (red solid line). Black dots are the corresponding experimental measurements. (c) Intensity distribution in the $z-r$ plane showing the free space evolution of the SGCR beam. (d) Intensity distribution of the SGCR beam normalized to the beam area to help visualizing the value of its confocal parameter $b$.

By further exploring the CR beam dependence with the $\rho_{0}$ parameter, we have found that at the value $\rho_{0}=0.445$ the transverse intensity profile of the transformed beam becomes flat at its top and it decays smoothly at its edges, i.e. it is a superGaussian conically refracted (SGCR) beam. To deduce such value of $\rho_{0}$, we solved Eqs. (2.12) and (2.13) numerically and we looked for which value of $\rho_{0}$ it is found a maximum number of points with a slope equal to zero at the transverse cross-section at the focal plane. Fig. 3.6(a) and Fig. 3.6(b) show, respectively, the cross-section of the transverse intensity profile of the input Gaussian beam and the output CR beam for 
$\rho_{0}=0.445$ along both the radial and the longitudinal directions. Fig. 3.6(c) plots the 2D intensity distribution of the SGCR beam in the $z-r$ plane, while Fig. 3.6(d) plots the corresponding intensity distribution normalized to the beam area. The plateau at the top part has been measured to be a $30 \%$ of the FWHM, which is compatible with a super-Gaussian beam of first order [103. Also, the depth of field of this super-Gaussian beam is larger than that one of a Gaussian beam, as shown in Fig. 3.6(b). In Gaussian beams, the depth of field or confocal parameter $b$ is twice the distance of the transverse plane at which the beam waist radius is $w\left(z_{R}\right)=\sqrt{2} w_{0}$, i.e. $b=2 z_{R}$. To obtain the confocal parameter for the SGCR beam, we solved numerically Eqs. (2.12) and (2.13) using $\rho_{0}=0.445$ and we looked for the axial distance $Z$ from the focal plane to which the area occupied by the SGCR beam was doubled with respect to the focal plane, i.e. we found $\mathrm{Z}$ accomplishing that $w(Z)=\sqrt{2} w(Z=0)$. The waist radius of beam, $w(Z)$, was considered at $e^{-2}$ of the maximum intensity at each plane. For the SGCR, we have found that the depth of field is $b_{\mathrm{SGCR}}=6.1 z_{R}$, as depicted in Fig. 3.6(d). Therefore, the SGCR beam reported here has a confocal parameter three times larger than that one of the fundamental Gaussian beam.

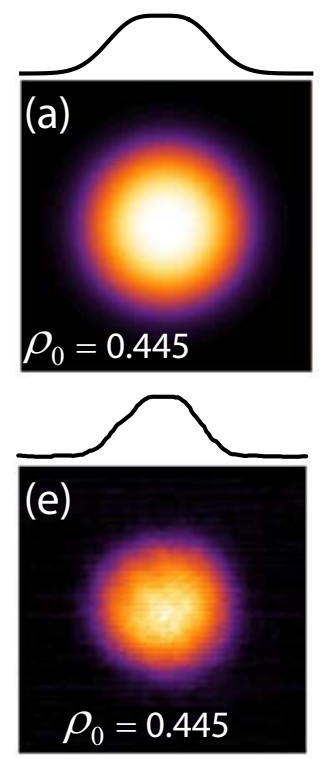

$\mathrm{Z}=0$
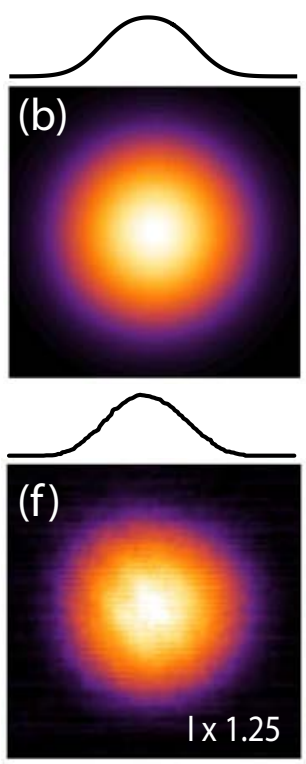

1
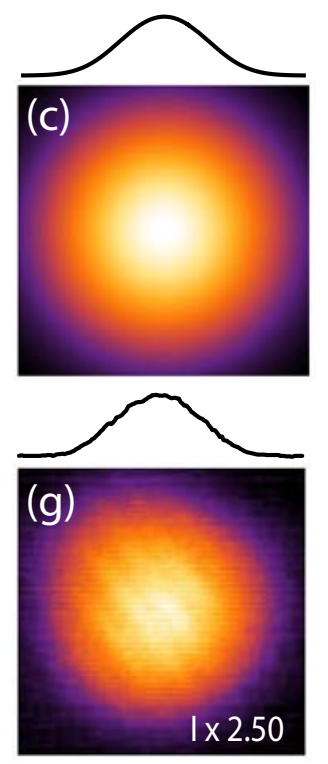

2

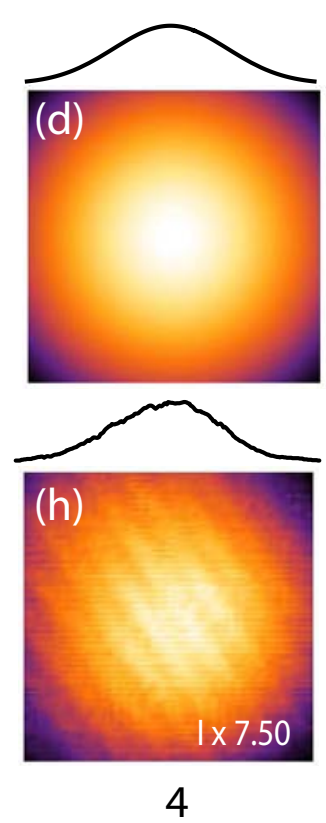

Figure 3.7: Transverse patterns along the beam propagation direction of the SGCR beam calculated theoretically (top row), and obtained experimentally (bottom row) for an input beam of transverse Gaussian profile. Insets are the cross-section of the intensity distribution along the horizontal direction.

Top row of Fig. 3.7presents the theoretical transverse patterns of the SGCR beam at different planes along its propagation for a Gaussian input beam. Top insets demonstrate that the flat-top profile of the SGCR beams is only obtained at the focal plane while when the imaging plane is moved to other planes the plateau disappears and the profile 
tends to be Gaussian-like.

Experiments on the SGCR beam only require an input beam, a BC and an imaging system (CCD camera). We use the same input beam and biaxial crystal as in the previous Section and a focusing lens with focal length of $200 \mathrm{~mm}$. The experimental value of the control parameter of $\rho_{0}$ obtained is 0.44 . Bottom row of Fig. 3.7 shows the experimental transverse patterns obtained at different propagation distances for the SGCR beams. Top insets represent the cross-section at the beam center along the horizontal direction. They agree well with the corresponding numerical simulations presented in Fig. 3.7. For this arrangement, we have also measured the confocal parameter obtaining that $b_{\mathrm{SGCR}}^{\exp }=(5.8 \pm 0.2) z_{R}$.

\subsection{Conclusions}

In conclusion, in this chapter we have carefully investigated CR both theoretically and experimentally in the regime $\rho_{0}=R_{0} / w_{0}<1$ for which novel intensity structures appear. On the one hand, for the specific value $\rho_{0}=0.924$, we have theoretically derived and experimentally reported the transformation of an input Gaussian beam into a bottle beam with a point of exact null intensity. At variance with spatial light modulators (SLMs), where a significant amount of light is lost by diffraction, the here proposed method transfers all the input power into the bottle beam, as long as the facets of the crystal have dielectric coatings to avoid reflections and the doping elements that can be found in some particular crystals do not absorb input light power at the used frequency. The obtained bottle beam could be useful for applications such as laser drilling, stimulated emission depletion microscopy, or trapping neutral atoms and Bose-Einstein condensates (BECs) by means of the light dipole force. An additional advantage is the fact that the quality and smoothness of the 3D dark focus obtained by means of CR is only limited by the quality of the input beam and the focusing lenses, while for SLMs it strongly depends on the pixel density. Nonetheless, this technique can be used in all the spectral range to which the biaxial crystal is transparent, at variance with other techniques such as computer generated holograms with SLMs or diffractive optical elements. Furthermore, as it is shown in Figs. 3.4(b) and (c), the bottle beam generated with $\mathrm{CR}$ can be adiabatically transformed into a 3D dark ring, by simply tuning the focusing geometry. Hence, it could be possible to trap a BEC in the 3D dark focus and then adiabatically transfer it into the dark ring to investigate, for instance, matter wave (Sagnac) interferometry or the appearance of persistent currents.

On the other hand, we have demonstrated that the CR phenomenon can be used as a tool for generating beams with Super-Gaussian transverse profiles, this means flat-top beams with a smooth decay at their edges, from input beams with transverse Gaussian profile. We have reported that the generation of the SGCR beams is governed by the adjustment of the control parameter $\rho_{0}$ to a value of 0.445 . We have shown that the SGCR beams have only the flat-top profile at the focal plane of the system, while far 
from the focal plane the transverse cross-section becomes Gaussian-like. Additionally, the SGCR beams have been shown to have a confocal parameter of $6.1 z_{R}$, i.e. more than three times that one for Gaussian beams. In addition to full power conversion, broad wavelength range and smoothness of the generated beam commented in the previous paragraph, the SGCR beam has the advantage presented that biaxial crystals can be used with high power beams, which is a requirement for high intensity x-rays experiments, for instance. One possible drawback of the presented method is the state of polarization of the SGCR beam, which is non-uniform and contains circular, elliptical and linear SOPs. However, the SOP of the beam is, in principle, only relevant in optical processing with tightly focused beams, while for the rest of the possible applications the most relevant aspect is the shape of the beam. The state of polarization and the optical singularities generated with low values of $\rho_{0}$ will be addressed in Chapter 4 . Finally, note that the methods presented in this Chapter could be extended to alternative input beams such as elliptical beams, which have been studied in detail in CR [54], to generate elliptical beams with transverse flat-top cross-section. 
CHAPTER 4

Novel vector beams and optical singularities in conical refraction

In this Chapter we report novel vector beams, i.e., beams with involving states of polarization (SOP), obtained by transforming a Gaussian beam passing through a biaxial crystal, by means of the conical refraction phenomenon. We analyze both experimentally and theoretically the SOP of the different vector beams generated and demonstrate that the SOP of the input beam can be used to control both the shape and the SOP of the transformed beam. We also identify polarization singularities of such beams and relate them to the spin-orbit coupling provided by the biaxial crystal and discuss them in terms of the coherent addition of two contributions, the $B_{0}$ and $B_{1}$ fields of the Beksly-Khapalyuk-Berry solution, which form the conical refraction beam.

The Chapter is organized as follows. In Sec. 4.1 we define vector beams, discuss their common applications and introduce our work. Then, Section 4.2 is devoted to briefly review the basics of the Stokes vector and CR formalisms needed for the subsequent sections and also to present separately the $B_{0}$ and $B_{1}$ components of the CR beam. In Sections 4.3 and 4.4, we report for $\rho_{0} \gg 1$ and $\rho_{0} \lesssim 1$, respectively, the generation of novel $\mathrm{CR}$ vector beams both theoretically and experimentally. We characterize these vector beams by measuring their Stokes parameters and propose methods to manipulate them as, for instance, making use of the SOP of the input beam. We also identify polarization singularities generated in CR beams and in Sec. 4.5 we relate them to the spin-orbit coupling provided by the biaxial crystal. Finally, in Sec. 4.6 we summarize the main results of this work and discuss potential applications of these CR vector beams to different fields of optics.

The research contained in this chapter has been done in collaboration with Alba Peinado, Ángel Lizana and Juan Campos from the Universitat Autònoma de Barcelona and has been published in Ref. 69]. 


\subsection{Introduction}

The state of polarization (SOP) is one of the fundamental signatures of light fields associated with their vectorial nature. In general, at each point in space the dynamics of the electric field vector of a wave can be described by an ellipse. This ellipse is known as polarization ellipse and it is characterized by the orientation of its major axis through the azimuth angle $\Phi \in[0, \pi]$ and by the ellipticity parameter $\beta \in[-\pi / 4, \pi / 4]$ so that $\tan \beta$ is the ratio of the axes of the polarization ellipse. If $\beta=0$ the light field is linearly polarized, while if $\beta= \pm \pi / 4$ the SOP will be circular (left handed for ' + ' and right handed for '-', if we consider an observer looking in the direction from which light is coming). Usually coherent light beams are homogeneously polarized, i.e. the SOP is identical for all points at any transverse plane along the beam propagation. However, there exist light beams possessing non-homogenous polarization, known as vector beams, such as the well known radial or azimuthal polarizations [101] or even beams with more involving polarization distributions [124 126]. The non-homogeneous polarization distribution of vector beams can lead to singular points where the SOP is exactly circular (C points), lines along which the SOP is linear (L lines) or disclinations where the instantaneous electric field is null [127-130]. Vector beams have been applied to laser material processing, optical imaging, atomic spectroscopy, and optical trapping (see [101] and the references therein), among many others.

In CR, for $\rho_{0} \gg 1$, the SOP of the beam at the focal plane is already well known [54, 59, 74, 131]. In this Chapter we investigate the CR SOP out of the focal plane, including values of $\rho_{0} \lesssim 1$, when Poggendorff fine splitting vanishes. By means of the Stokes vector formalism, we characterize the resulting novel vector beams of CR and show that the SOP of the input beam can be used to control both the shape and the $\mathrm{SOP}$ of the transformed CR beam.

\subsection{Theoretical background}

\subsubsection{Analysis of the $B_{0}$ and $B_{1}$ functions}

From Eqs. (2.11)-(2.13), for a homogeneously polarized input beam with state of polarization given by $\mathbf{e}_{\mathbf{0}}=\left(e_{x}, e_{y}\right)$, the electric field components in Cartesian coordinates $E_{x}$ and $E_{y}$ of the CR beam behind the biaxial crystal can be written as follows:

$$
\begin{aligned}
& E_{x}=\left(B_{0}+B_{1} \cos \varphi\right) e_{x}+\left(B_{1} \sin \varphi\right) e_{y}, \\
& E_{y}=\left(B_{1} \sin \varphi\right) e_{x}+\left(B_{0}-B_{1} \cos \varphi\right) e_{y},
\end{aligned}
$$


where $\phi$ is the azimuthal angle in cylindrical coordinates. From Eq. (4.1) and Eq. (4.2) it is straightforward to obtain the SOP of the $B_{0}$ and $B_{1}$ components:

$$
\begin{aligned}
& B_{0, x}=B_{0} e_{x}, \\
& B_{0, y}=B_{0} e_{y}, \\
& B_{1, x}=B_{1} \cos \varphi e_{x}+B_{1} \sin \varphi e_{y}, \\
& B_{1, y}=B_{1} \sin \varphi e_{x}-B_{1} \cos \varphi e_{y} .
\end{aligned}
$$
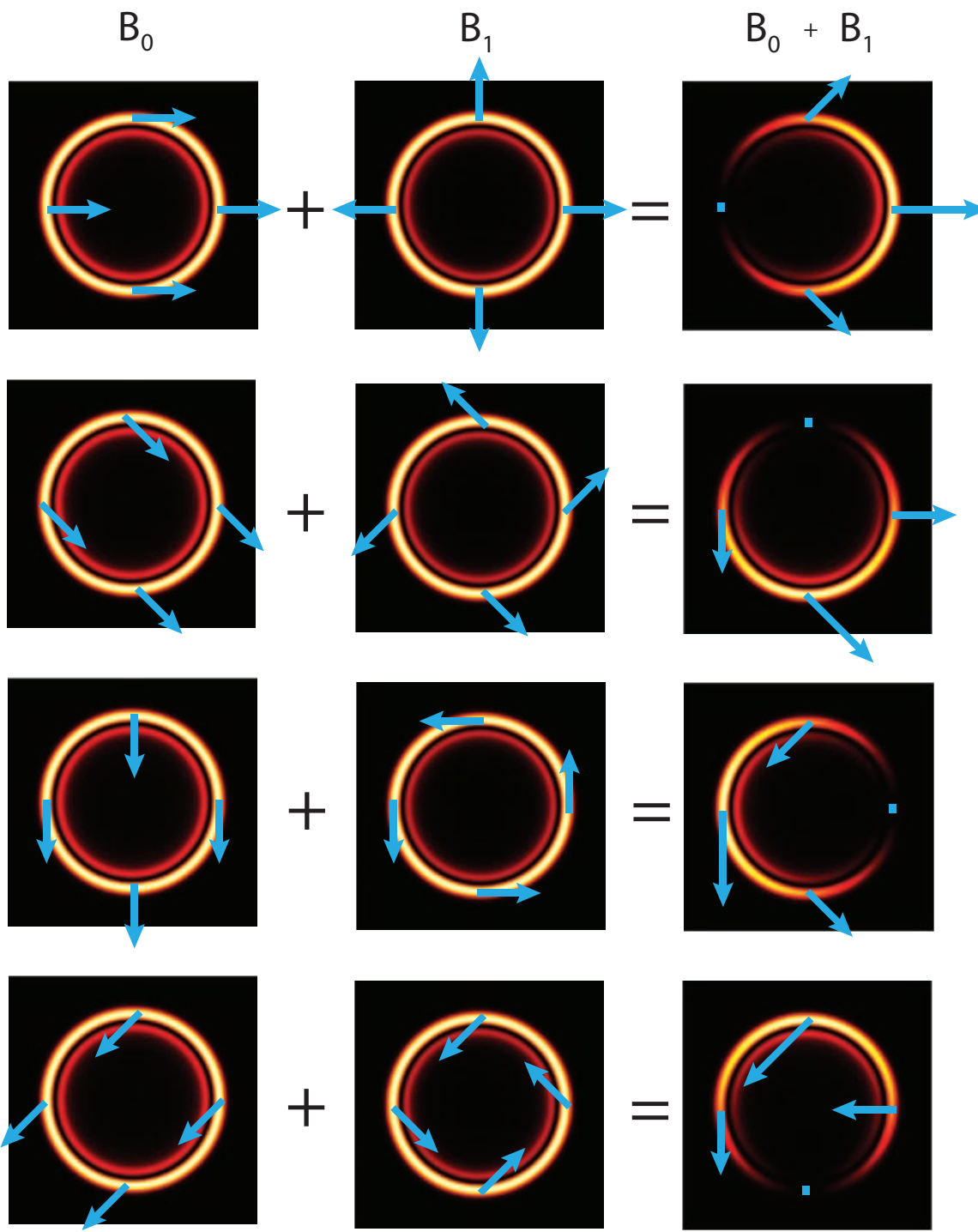

Figure 4.1: Transverse intensity patterns and electric field (blue arrows) of the $B_{0}$ component (first column), $B_{1}$ component (second row) and CR rings (third column) obtained at the focal plane for a right-handed circularly polarized Gaussian input beam. Each row represents a different moment of time over half a period of rotation of the electric field. $\rho_{0}=10$. 
For a circularly polarized Gaussian input beam with $\mathrm{SOP} \mathbf{e}_{\mathbf{0}}=(1, \pm i) / \sqrt{2}\left({ }^{+}+\right.$' for left-handed and '-' for right-handed) passing through the biaxial crystal under CR conditions, Eqs. (4.3)-(4.6) read as follows

$$
\begin{aligned}
B_{0, x} & =\frac{1}{\sqrt{2}} B_{0}, \\
B_{0, y} & =\frac{ \pm i}{\sqrt{2}} B_{0}, \\
B_{1, x} & =\frac{1}{\sqrt{2}} B_{1} e^{ \pm i \varphi}, \\
B_{1, y} & =\frac{\mp i}{\sqrt{2}} B_{1} e^{ \pm i \varphi} .
\end{aligned}
$$

For this case, the SOP of the $B_{0}$ and $B_{1}$ components can be easily understood. Both components are circularly polarized but, while the $B_{0}$ component is in phase with the input beam, the $B_{1}$ component is in counter-phase and possesses an azimuthal phase $e^{i \varphi}$ associated to beams carrying $\pm \hbar$ orbital angular momentum (OAM) per photon [132 135]. In other words, every point of the $B_{1}$ component is circularly polarized but there is a rotation of the field by $2 \pi$ on running along the azimuthal angle $\varphi$.

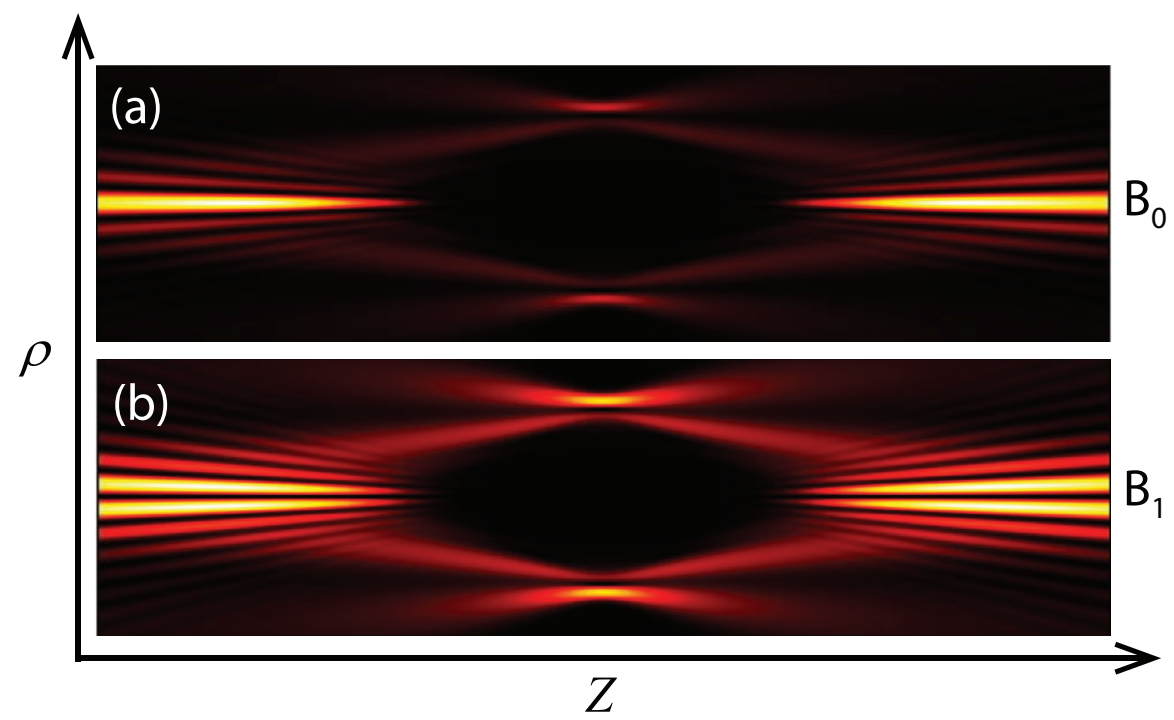

Figure 4.2: Transverse intensity cuts in the $Z-\rho$ plane of the (a) $B_{0}$ and (b) $B_{1}$ components.

The SOP of the $B_{1}$ component combined with the $B_{0}$ component leads to the characteristic CR polarization distribution along the CR rings obtained for $\rho_{0} \gg 1$, as shown in Fig. 4.1 for a right-handed circularly polarized (RHCP) Gaussian input beam. As it can be observed, the transverse intensity patterns at the focal plane of the $B_{0}$ (first column) and $B_{1}$ (second column) components are practically indistinguishable each other and almost identical to the CR pattern (third column), except for the presence of a 
node in the latter. Each row corresponds to different moments of time running over half a period, the blue arrows indicating the SOP at different points of the rings. This sequence of images corroborate the previous highlighted features of the electric field of the $B_{0}$ and $B_{1}$ components: (i) electric field of the $B_{0}$ component rotates clockwise, as the input beam, while the electric field of the $B_{1}$ component rotates counter-clockwise; and (2) all the points of the $B_{0}$ component are in phase whereas for the $B_{1}$ component there is an azimuthal phase of $2 \pi$ along the rings. Note that at any moment of time the transverse intensity pattern of the $B_{0}$ and $B_{1}$ components form complete light rings. In contrast, the $\mathrm{CR}$ transverse intensity patterns form a azimuthally crescent annular structures with the position of the null-intensity point rotating in counter-phase with the input beam. From these images it is clear that how SOP of the CR rings is obtained from the coherent addition of the $B_{0}$ and $B_{1}$ fields.

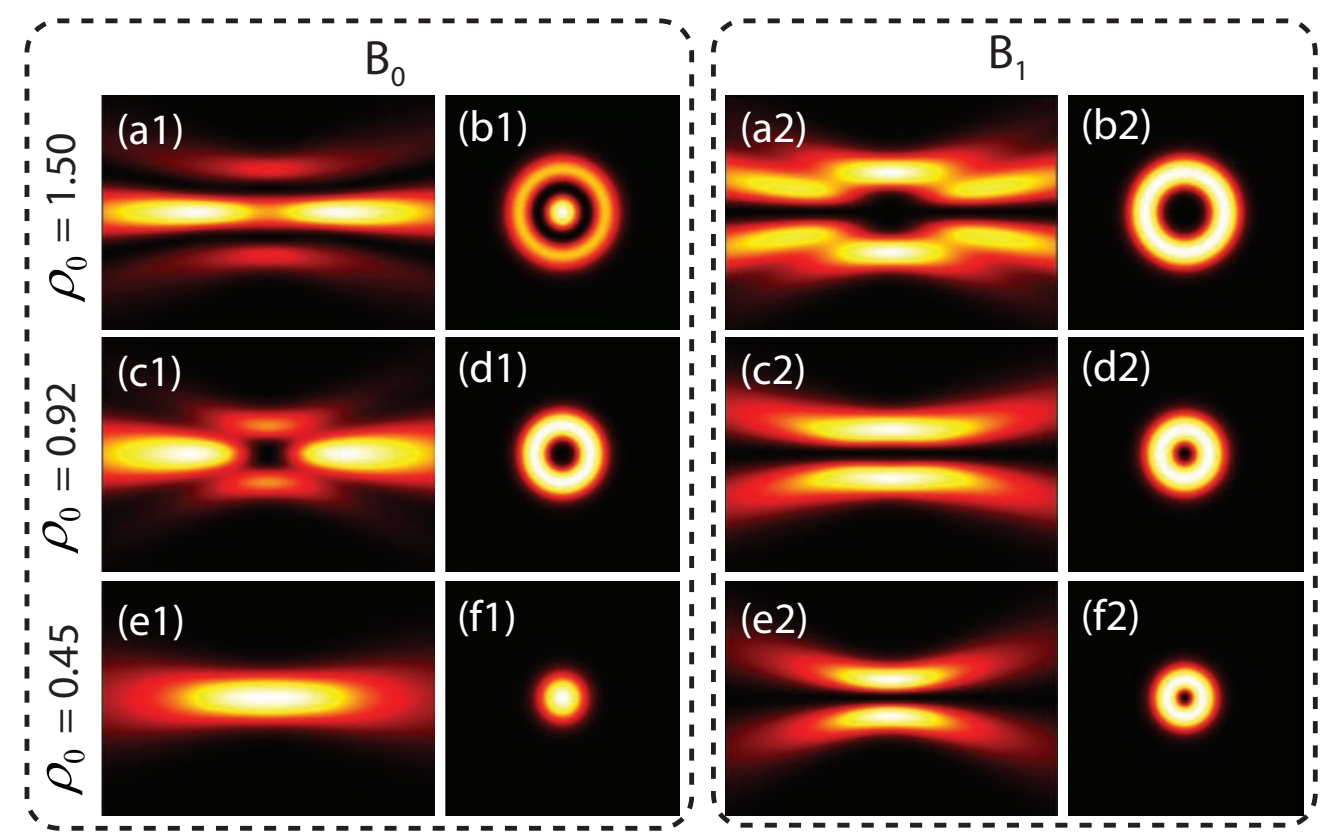

Figure 4.3: $B_{0}$ (left-hand side box) and $B_{1}$ (right-hand side box) beams obtained for $\rho_{0}=1.50$ (first row), $\rho_{0}=0.92$ (second row) and $\rho_{0}=0.45$ (third row) in the $Z-\rho$ plane (first column of each box) and in $X-Y$ plane at $Z=0$ (second column of each box).

As shown in Fig. 4.1 the transverse intensity patterns of the $B_{0}$ and $B_{1}$ fields at the focal plane $(Z=0)$ are almost identical. However, out of the focal plane the situation changes, see Fig. 4.2. In particular, at the positions where the Raman spots would start to form for the whole CR beam, the nature of the $J_{0}$ and $J_{1}$ Bessel functions involved in the $B_{0}$ and $B_{1}$ components becomes appreciable. Far enough from the focal plane, the $B_{0}$ beam possesses an axial intensity maximum, while the $B_{1}$ beam has a nodal line coinciding with the $Z$ axis, i.e., it forms an optical vortex, which is an optical singularity [128. This result becomes more appreciable for low values of $\rho_{0}$. In Fig. 4.3 
we show the intensity patterns of the $B_{0}$ (left-hand side box) and $B_{1}$ (right-hand side box) components for $\rho_{0}=1.50$ (first row), $\rho_{0}=0.92$ (second row) and $\rho_{0}=0.45$ (third row) in the $Z-\rho$ plane (first column of each box) and in $X-Y$ plane at $Z=0$ (second column of each box). It is clear that the $B_{1}$ field forms an optical vortex possessing a nodal line along the $Z$ axis, while the $B_{1}$ component has, in general, light intensity at the beam axis. These results obtained by analyzing separately the $B_{0}$ and $B_{1}$ components of the CR beam will be very useful to understand the vector beams and optical singularities shown in the following Sections.

\subsubsection{Stokes vector formalism}

Since in this Chapter we aim to analyze in detail the SOP of CR beams, in what follows we introduce the standard tool to analyze the SOP of a light beam: the Stokes vector $\mathbf{S}=\left(S_{0}, S_{1}, S_{2}, S_{3}\right)$. For an electric field $\mathbf{E}=\left(E_{x}, E_{y}\right)$ with intensity $I$ the Stokes parameters read [6]:

$$
\begin{aligned}
& S_{0}=I=\left|E_{x}\right|^{2}+\left|E_{y}\right|^{2}, \\
& S_{1}=I_{0^{\circ}}-I_{90^{\circ}}=\left|E_{x}\right|^{2}-\left|E_{y}\right|^{2}, \\
& S_{2}=I_{45^{\circ}}-I_{135^{\circ}}=2 \operatorname{Re}\left[\mathrm{E}_{\mathrm{x}}^{*} \mathrm{E}_{\mathrm{y}}\right], \\
& S_{3}=I_{R}-I_{L}=2 \operatorname{Im}\left[\mathrm{E}_{\mathrm{x}}^{*} \mathrm{E}_{\mathrm{y}}\right],
\end{aligned}
$$

where $I_{\Phi}\left(\Phi=0^{\circ}, 45^{\circ}, 90^{\circ}, 135^{\circ}\right)$ indicates the intensity of linearly polarized light with azimuth $\Phi$, and $I_{R}$ and $I_{L}$ indicate the intensity of right- and left-handed circularly polarized light, respectively. In what follows, we use equations normalized to $E^{2}$, i.e. we consider $I=E^{2}=1$. These definitions show that $S_{0}$ account for the intensity of the light beam, $S_{1}$ measures the amount of light which is linearly polarized (LP) in the vertical/horizontal basis, $S_{2}$ does the same but with the diagonal basis and $S_{3}$ relates the state of polarization in the right- and left- circularly polarized (CP) basis. The following equations show how the Stokes parameters account for the azimuth $\epsilon$ and ellipticity $\beta$ of the polarization ellipse [6]:

$$
\begin{aligned}
\epsilon & =\frac{1}{2} \arctan \left(\frac{S_{2}}{S_{1}}\right) \\
\beta & =\frac{1}{2} \arctan \left(\frac{S_{3}}{\sqrt{S_{1}^{2}+S_{2}^{2}}}\right) .
\end{aligned}
$$

\subsection{State of polarization for $\rho_{0} \gg 1$}

The first information that can be extracted from Eqs. (2.11)-(2.13) with respect to the SOP of the CR beam is that at $\rho=0$ there is only contribution of $B_{0}$, since $B_{1} \propto J_{1}(\eta \rho=0)=0$. Additionally, as commented previously, the SOP of the $B_{0}$ component is $\mathbf{e}_{0}$. As a consequence, the center of the $\mathrm{CR}$ beam will possess always the 
same SOP as the input beam. This fact, that was already pointed out in [62,63,65], will be discussed with more detail below. To obtain the Stokes parameters of the CR beam, Eqs. (4.11) -(4.14) must be combined with Eqs. (2.11)-(2.13). For a circularly polarized $(\mathrm{CP})$ input beam, the electric field and intensity beyond the crystal become:

$$
\begin{aligned}
E_{x} & =B_{0}+B_{1} e^{ \pm i \varphi}, \\
E_{y} & = \pm i B_{0} \mp i B_{1} e^{ \pm i \varphi}, \\
I_{\mathrm{CP}} & =2\left(\left|B_{0}\right|^{2}+\left|B_{1}\right|^{2}\right),
\end{aligned}
$$

where upper/lower sign stays for LHCP/RHCP beam. For a linearly polarized (LP) input beam, the corresponding electric field and intensity beyond the crystal read as follows:

$$
\begin{aligned}
E_{x} & =B_{0} \cos \Phi+B_{1} \cos (\varphi-\Phi), \\
E_{y} & =B_{0} \sin \Phi+B_{1} \sin (\varphi-\Phi), \\
I_{\mathrm{LP}} & =I_{\mathrm{CP}}+2 \operatorname{Re}\left[B_{0} B_{1}^{*}\right] \cos (2 \Phi-\varphi),
\end{aligned}
$$

where $\Phi$ is the polarization azimuth of the LP input beam with $\mathbf{e}_{\mathbf{0}}=(\cos \Phi, \sin \Phi)$.

For well resolved concentric rings with Poggendorff splitting, i.e. for $\rho_{0} \gg 1$, Eqs. (2.12), (2.13), (4.19) and (4.22) show that a radially symmetric intensity pattern of CR is obtained only for a CP input beam. Instead, for a LP input beam, a crescent annular intensity pattern appears such that the zero intensity point is obtained for the ring position that possesses orthogonal polarization to the input beam. In both cases, the polarization distribution of the CR pattern is the same. Every point of the rings is linearly polarized and the azimuth rotates continuously along the ring so that every two diametrically opposite points have orthogonal polarizations.

Fig. 4.4(a) presents the numerically obtained Stokes parameters at transverse sections of the CR beam $\left(\rho_{0}=10\right)$ at $Z=0$ (first and second rows) and $Z=10.92$ (third and fourth rows) obtained from a RHCP (first and third rows) and a $\operatorname{LP}\left(\Phi=45^{\circ}\right)$ (second and fourth rows) Gaussian input beam. At the focal plane, see first two rows in Fig. 4.4 (a), the SOP described by the Stokes parameters is the expected: symmetric pattern for the RHCP case and with a node at $\varphi=270^{\circ}\left(\Phi=135^{\circ}\right.$, since we have used $\left.\varphi_{C}=0^{\circ}\right)$. Last column in Fig. 4.4(a) demonstrates that at the focal plane the SOP of the CR beams, either RHCP or LP $\left(\Phi=45^{\circ}\right.$ ) (or any other) is linear, i.e. $S_{3}=0$. In contrast, for the Raman spot, $Z=10.92$, all Stokes parameters are substantially different from zero, as shown in the last two rows in Fig. 4.4(a).

Stokes parameters in Fig. 4.4(a) clearly identify polarization singularities of CR beams. For RHCP input light, it is a C-point at the center of the CR beam, i.e., it is of $\mathrm{CP}$ at any point of the beam center along propagation. The center of the $S_{3}$ transverse pattern is a point with maximum intensity, while the other two Stokes parameters $S_{1}$ and $S_{2}$ have zero values. For $\mathrm{LP}_{45^{\circ}}$ input light, one can identify L-line singularity. In Fig. 4.4(a) this line can be identified as a vertical line of zero value of the Stokes 
parameters $S_{3}$ and $S_{1}$ and nonzero value of $S_{2}$. It belongs to the plane defined by the points of CR ring with linear $\mathrm{SOP}$ of $\mathrm{LP}_{\Phi=45^{\circ}}$ and $\mathrm{LP}_{\Phi=135^{\circ}}$.

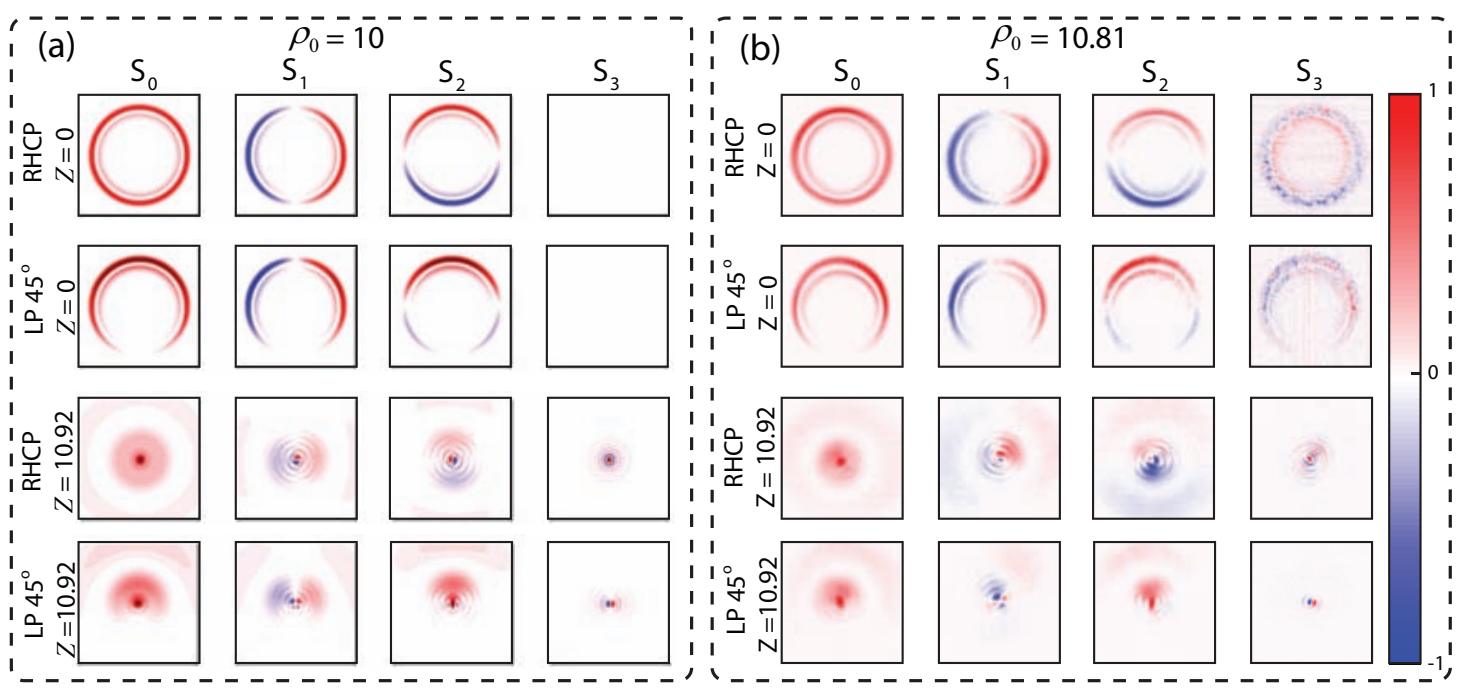

Figure 4.4: (a) Theory: transverse pattern for $\rho_{0}=10$ of the Stokes parameters $S_{0}, S_{1}, S_{2}, S_{3}$ obtained from numerical simulations for the CR beam transverse profile with a RHCP and a LP $\left(\Phi=45^{\circ}\right)$ Gaussian input beam. First and second rows correspond to the focal plane $(Z=0)$ while third and fourth rows to the Raman spot plane $(Z=10.92)$. The plane of optic axes of the crystal lies horizontally $\left(\varphi_{c}=0\right)$. (b) Experiment: for $\rho_{0}=10.81$, transverse patterns of the Stokes parameters $S_{0}, S_{1}, S_{2}, S_{3}$ for the CR beams measured with a RHCP and a LP $\left(\Phi=45^{\circ}\right)$ Gaussian input beam. First and second rows correspond to the focal plane $(Z=0)$ while third and fourth rows to the Raman spot plane $(Z=10.92)$.

We have also investigated the spatial evolution of the Stokes parameters along the propagation direction for a RHCP and LP $\left(\Phi=45^{\circ}\right)$ Gaussian input beam. As we move away from the focal plane, the bright rings become wider and the intensity at the Poggendorff dark ring is no longer zero. The intensity of the outer ring decreases while the intensity of the inner ring increases. The outer ring expands and the inner ring becomes smaller in radius. As a result, at $Z \approx 6$ a spot in the center of the pattern appears. Finally, the inner ring shrinks into a bright spot at $Z=10.92$ for $\rho_{0}=10$, corresponding to the Raman spot. At this point, it can be found an additional type of polarization singularity independently on the polarization state of an input beam. It is clearly distinguishable by inspection of the Stokes parameter $S_{3}$ that defines degree of circular polarization of the field. Observing the $S_{3}$ parameter far from the focal plane, for instance, at the Raman spot as shown in the last two rows in Fig. 4.4(a), reveals alternating annular regions of RHCP and LHCP states. These annular regions of circular polarization are separated by circles of null intensity. At these circles the field is linearly polarized. Therefore, these polarization singularities can be called as L- 
circles. This behavior has been also observed in the focusing of radially polarized beams and explored in detail in Refs. [136, 137]. For a LP $\left(\Phi=45^{\circ}\right)$ input beam, the central spot is broken by a line of null intensity (L-line) that connects the two points with LP and azimuth $\Phi=45^{\circ}$ and $\Phi=135^{\circ}$, as commented before. Note that polarization singularities, i.e., C-point for CP input beam, L-lines for LP input beam and L-circles, are invariants of $\mathrm{CR}$ beam propagation behind the crystal.

To test the validity of the obtained theoretical results, we have performed the corresponding experimental measurements. Fig. 4.5 shows the experimental set-up. The Gaussian input beam is obtained from a diode laser at $640 \mathrm{~nm}$ coupled to a monomode fiber with a collimator, yielding a beam waist of $w_{0}=1.26 \mathrm{~mm}$. To generate the different input polarization states (LP with $\Phi=45^{\circ}$ and RHCP) we use half- and quarterwaveplates. We use lenses with different focal lengths to modify the waist radius $w_{0}$ of the input beam and to reach different values of $\rho_{0}$. The beam passes along one of the optic axes of a biaxial crystal, placed always before the expected focal plane of the beam. An additional imaging lens is used to take different planes along the beam propagation direction and transfer them onto the CCD camera. Linear and circular polarizers are used to measure the Stokes parameters of the beam after being transformed by the CR phenomenon. We use a commercially available (CROptics) $\mathrm{KGd}\left(\mathrm{WO}_{4}\right)_{2}$ biaxial crystals with $\alpha=16.9 \mathrm{mrad}$ and length $l=10.5 \mathrm{~mm}$ yielding $\mathrm{CR}$ ring radius of $R_{0}=180 \mu \mathrm{m}$.

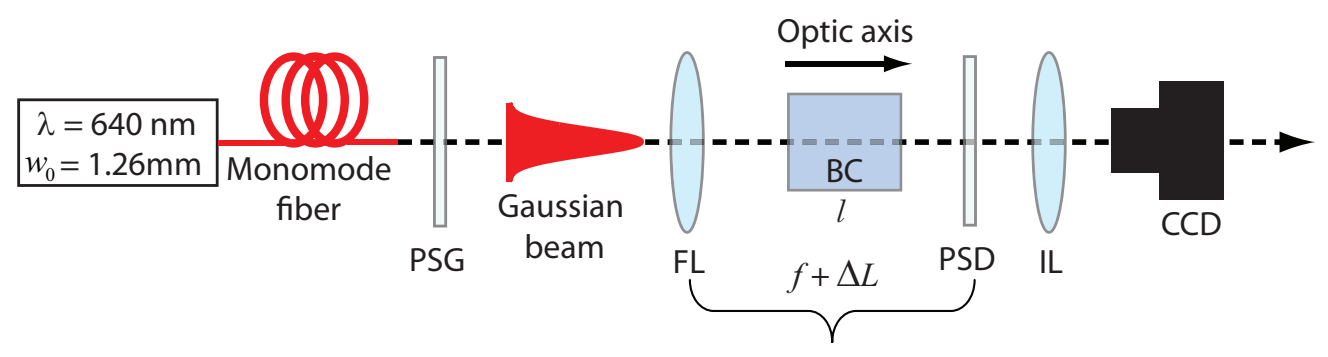

Figure 4.5: Experimental set-up. A diode laser coupled to a monomode fiber generates a Gaussian beam at $640 \mathrm{~nm}$ with a beam waist radius $w_{0}=1.26 \mathrm{~mm}$. Then the beam is focused by means of a focusing lens (FL) along one of the optic axes of a $\mathrm{KGd}\left(\mathrm{WO}_{4}\right)_{2}$ biaxial crystal (BC). Experiments from Fig. 4.4 were carried out using a FL with $100 \mathrm{~mm}$ focal length and a biaxial crystal $10.5 \mathrm{~mm}$ long, while FLs with focal lengths of $150 \mathrm{~mm}$, $200 \mathrm{~mm}$ and $400 \mathrm{~mm}$ and a biaxial crystal $2.3 \mathrm{~mm}$ long were used for the experiments from Fig. 4.7. Linear and circular polarizers are used as polarization state generators (PSG) and polarization state detectors (PSD) to generate and measure the SOP of the input and output beam, respectively. The transverse patterns are recorded by means of an imaging lens (IL) that projects the image into a CCD camera.

Fig. 4.4(b) shows the obtained experimental Stokes parameters for $\rho_{0}=10.81$. The experimental results are in good agreement with the theoretical results presented in Fig. 4.4(a). Discrepancy has been observed only for the $S_{3}$ parameter for the case of a 
LP input beam (see last image of second rows) of Fig. 4.4(a). This can be explained in terms of the experimental error introduced by the polarization state detector elements used, that disturb the beam shape and its position, which is central for the quality of the experimental results.

\subsection{State of polarization for $\rho_{0} \lesssim 1$}
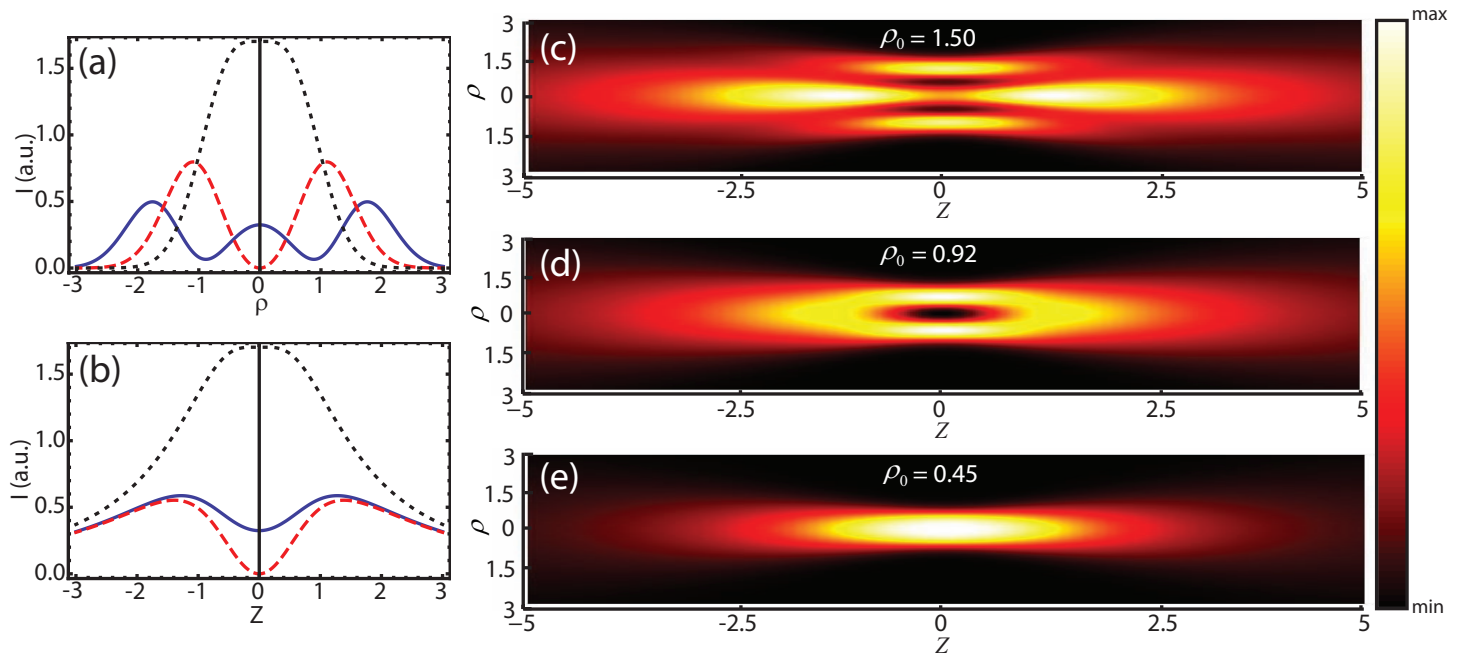

Figure 4.6: Intensity variation (a) along the radial direction $\rho$ at the focal plane $Z=0$ and (b) along the axial direction $Z$ at the beam center $(\rho=0)$ for CR vector beams obtained using $\rho_{0}=1.50$ (blue-solid line), $\rho_{0}=0.92$ (red-dashed line) and $\rho_{0}=0.45$ (black-dotted line). The corresponding intensity distribution in the $(Z, \rho)$ plane are shown in figures (c)-(e)

For $\rho_{0} \lesssim 1$ CR patterns are significantly different from the double bright concentric rings with clear Poggendorff splitting (occurring for $\rho_{0} \gg 1$ as shown in the previous section). The region $\rho_{0} \lesssim 1$ has been explored recently [63,66, 68] showing that CR can be used to design new CR lasers [138], to increase the directivity of laser beams [63, to generate a super-Gaussian beam [68, to create a three dimensional dark focus [66] and even to develop a novel scheme for super-resolution microscopy [85]. However, in all these works, no deep insight about the SOP of the generated CR beams has been provided. In what follows by considering the Stokes parameters we uncover the evolution of the SOP and polarization singularities of the CR beams and demonstrate how they depend on the SOP of the input beam. Fig. 4.6 shows the main features and general view of CR beams with $\rho_{0}=[1.50,0.92,0.45]$. The cross-section of the CR transverse intensity pattern at the focal plane and far away from the focal plane are shown in Figs. 4.6(a) and (b), respectively. Figs. 4.6(c)-(e) are 2D density plots of the intensity of the CR beams in the $Z-\rho$ plane. 
Figs. 4.7(a), (c) and (e) presents density plots of the numerically calculated Stokes parameters for (a) $\rho_{0}=1.50$, (c) $\rho_{0}=0.92$ and (e) $\rho_{0}=0.45$ at $Z=0$ (first and second rows) and out of the focal plane (third and fourth rows). For $\rho_{0}=1.50$ and $\rho_{0}=0.92$ these planes give the axial intensity maximum, while for $\rho_{0}=0.45$ we have considered the plane where the cross-section area of the beam is doubled [68]. First and third rows refer to a RHCP Gaussian input beam while second and fourth rows present the case of a $\operatorname{LP}\left(\Phi=45^{\circ}\right)$ Gaussian input beam. For $\rho_{0} \lesssim 1$ the position of the Raman spots are not well determined by Eq. (2.18) and the position of the axial intensity maxima must be determined for each particular case of $\rho_{0}$. Its $Z$ position is indicated at each image. The corresponding experimentally measured transverse Stokes parameters are shown in Figs. 4.7(b), (d) and (f) for (b) $\rho_{0}=1.48$, (d) $\rho_{0}=0.95$ and (f) $\rho_{0}=0.45$ at $Z=0$ (first and second rows) and out of the focal plane (third and fourth rows). For these experiments, the same set-up shown in Fig. 4.5 was used but in this case taking a $2.3 \mathrm{~mm}$ long $\mathrm{KGd}\left(\mathrm{WO}_{4}\right)_{2}$ biaxial crystal yielding $\mathrm{CR}$ ring radius of $R_{0}=39 \mu \mathrm{m}$.

While transverse distribution for intensity and polarization of CR beam look different for the $\rho_{0} \lesssim 1$ case with respect to $\rho_{0} \gg 1$, it should be noted that singularities, i.e., the topological structure of the CR beam, remains similar. It means that (i) the CR beam center constitutes a C-point singularity for the case of RHCP input beam (see the first and third rows in Fig. 4.7), (ii) there exists a L-line polarization singularity for the $\mathrm{LP}_{\Phi=45^{\circ}}$ input beam (see the second and fourth rows in Fig. 4.6) and (iii) there exists a L-circle singularity for input beam with arbitrary SOP (see the third and fourth rows in Fig. 4.7).

If we consider the special case of $\rho_{0}=0.92$, the central point at the focal plane is a null-intensity point and therefore all Stokes parameters are also 0 at the beam center. Vanishing intensity at the beam center leads to another feature: at the focal plane the sign of the circular polarization state associated with the Stokes parameter $S_{3}$ is the opposite with respect to the input beam. In other words, at the focal plane the CR beam is predominantly LHCP for the RHCP input beam that we consider in this article (see first row in Fig. 4.7 for the case of $\rho_{0}=0.92$ ). This feature is easily deduced from the mathematical formulation, since for this $\rho_{0}$ the value of the integral $B_{0}$ at any radial point is null, which leads to dominance of the contribution of integral $B_{1}$ associated with a SOP orthogonal to the input one, see Fig. 4.3. For the case of a LP $\left(\Phi=45^{\circ}\right)$ input beam and for all values of $\rho_{0}$ investigated, at the focal plane $Z=0$ the transverse patterns are crescent-like, with the intensity minimum at a point diagonally opposite to the point with maximum intensity. In the case of $\mathrm{LP}_{\Phi=45^{\circ}}$ input beam that we consider, the intensity minimum is observed at the bottom, corresponding to the point of LP with $\Phi=135^{\circ}$. In contrast to the RCHP case, for all three values of $\rho_{0}$ the $S_{3}$ parameter is null, which means that the patterns are completely LP. Out of the focal plane $S_{3} \neq 0$ except at the beam center, where there is an L-line connecting the points with $\operatorname{LP} \Phi=45^{\circ}$ and $\Phi=135^{\circ}$. 

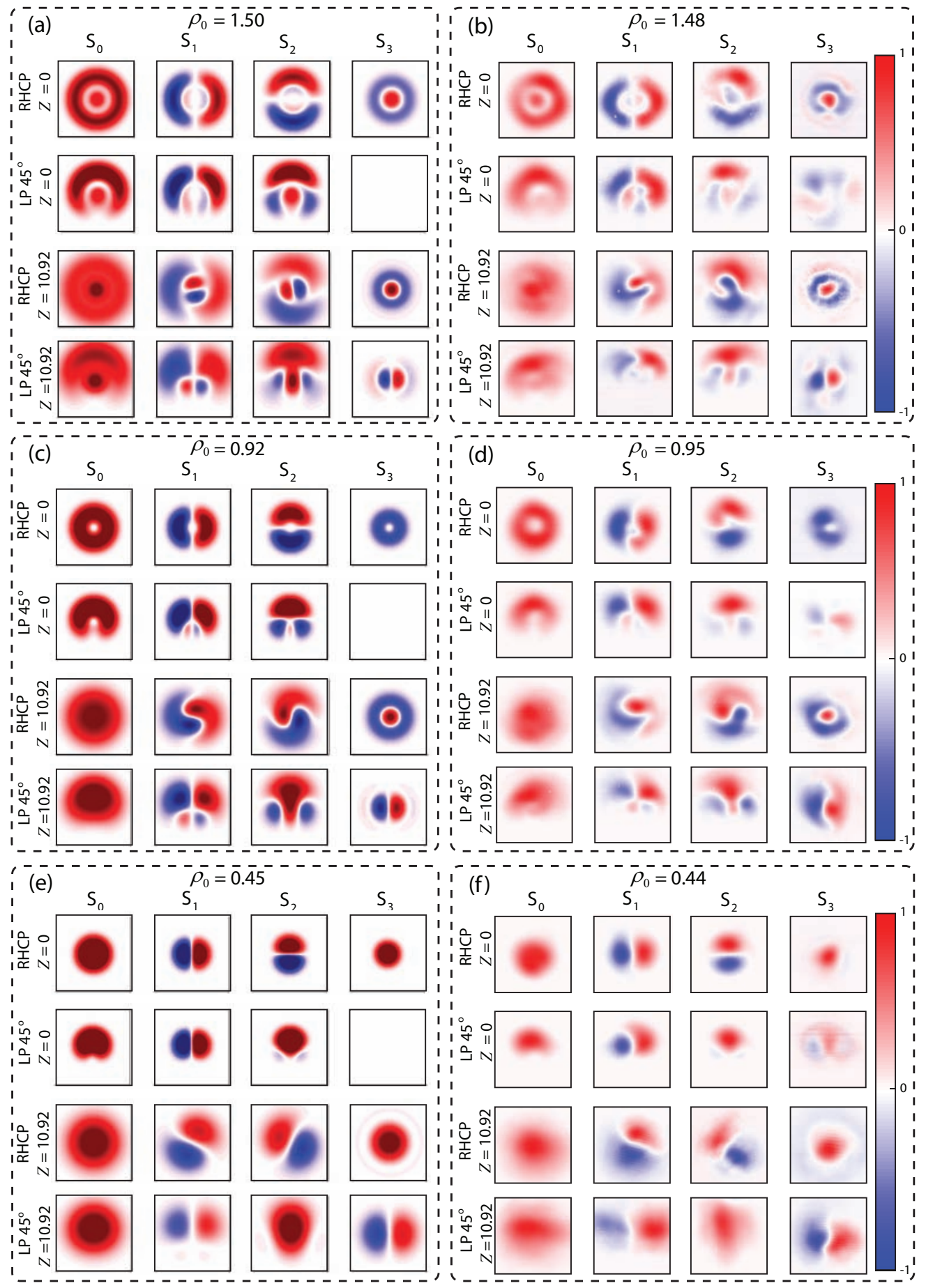

Figure 4.7: Numerically calculated Stokes parameters for $\rho_{0}=1.50$ (a), $\rho_{0}=0.92$ (c), and $\rho_{0}=0.45$ (e); and measured Stokes parameters for: (b) $\rho_{0}=1.48$, (d) $\rho_{0}=0.95$, and (f) $\rho_{0}=0.44$. The plane of optic axes of the crystal lies horizontally $\left(\varphi_{c}=0\right)$.

The theoretical predictions are, in general, in good agreement with the experimental 
results. Again, the $S_{3}$ parameter for a LP input beam (last image of second and fourth rows of Figs. 4.7(a), (c) and (e)) is the measurement that differs most with respect to the numerical predictions. In addition to the experimental difficulties commented above, i.e., the experimental error introduced by the polarization state detector elements used that disturb the beam shape and its position (being these ones particularly significant for large values of $w_{0}$ ), here it must be also taken into account the fact that small changes in the $\rho_{0}$ can modify quantitatively the CR pattern. Additional features of focused CR beams are associated with Gouy phase.

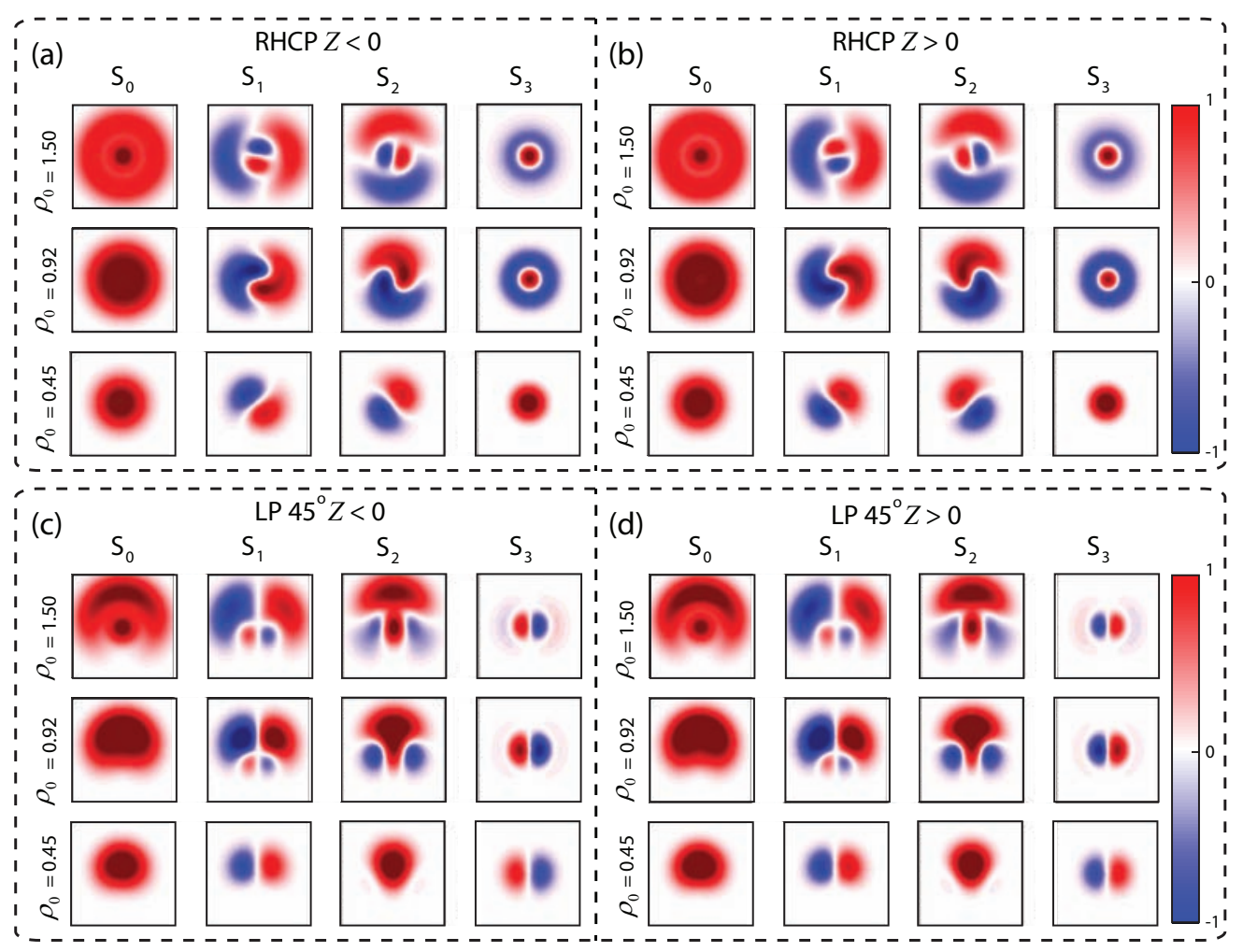

Figure 4.8: Numerically calculated Stokes parameters for $\rho_{0}=1.50$ (top row of each box), $\rho_{0}=0.92$ (middle row of each box), and $\rho_{0}=0.45$ (bottom row of each box) before $(\mathrm{a}, \mathrm{c})$ and after $(\mathrm{b}, \mathrm{d})$ the focal plane of a right-handed circularly polarized (RHCP) beam and a linearly polarized (LP) beam with azimuth at $45^{\circ}$.

It can be revealed by considering the transverse pattern evolution for Stokes parameters along CR beam propagation shown in Fig. 4.8 for a RHCP (top boxes) and LP $\left(\Phi=45^{\circ}\right)$ (bottom boxes) Gaussian input beam for $\rho_{0}=1.50, \rho_{0}=0.92$ and $\rho_{0}=0.45$ before $(Z<0)$ and after $(Z>0)$ the focal plane. For RHCP input light [boxes (a) and (b)] the focal plane is a symmetry plane for $S_{3}$. In contrast, $S_{1}$ and $S_{2}$ are rotated roughly $180^{\circ}$ before and after the focal plane, which must be associated to the Gouy phase [139]. For a LP $\left(\Phi=45^{\circ}\right)$ input beam [boxes (c) and (d)] the Stokes parameters $S_{1}$ and $S_{2}$ are symmetric with respect to the focal plane and now $S_{3}$ suffers from a phase 
shift of $180^{\circ}$, due to the Gouy phase too.

\subsection{Discussion in terms of spin-orbit coupling}

In general, the rotation of the Stokes parameters before and after the focal plane obtained for CR beams is also observed with focused Gaussian beams: due to the Gouy phase shift, which induces an additional phase in the electric field of $180^{\circ}$ at the focal plane, the $S_{1}$ and $S_{2}$ of a Gaussian beam rotate around the $Z$ axis by $180^{\circ}$. However, note that at variance with the results expected for a focused Gaussian beam, the $S_{1}$ and $S_{2}$ parameters of CR beams obtained from CP input beams not only rotate along the $Z$ axis but also twist around it at the focal plane. CR beams possess non-integer OAM that is generated due to the spin-orbit coupling provided by the biaxial crystal, as it has been reported by Berry and co-workers [140]. In Ref. [140] it is shown that the total OAM $J_{\mathrm{OAM}}$ and total spin angular momentum (SAM) $J_{\mathrm{SAM}}$ carried by the CR beam as a function of $\rho_{0}$ and of the initial total angular momentum $J_{0}$ are:

$$
\begin{aligned}
J_{\mathrm{OAM}} & =J_{0} \frac{1}{2} \rho_{0} e^{-\rho_{0}^{2}} \sqrt{\pi} D\left(\rho_{0}\right) \\
J_{\mathrm{SAM}} & =J_{0}\left(1-\rho_{0} e^{-\rho_{0}^{2}} \sqrt{\pi} D\left(\rho_{0}\right)\right)
\end{aligned}
$$

where $D(x)$ is the Dawson integral

$$
D(x)=\frac{2}{\sqrt{\pi}} \int_{0}^{\infty} d t e^{t^{2}}
$$

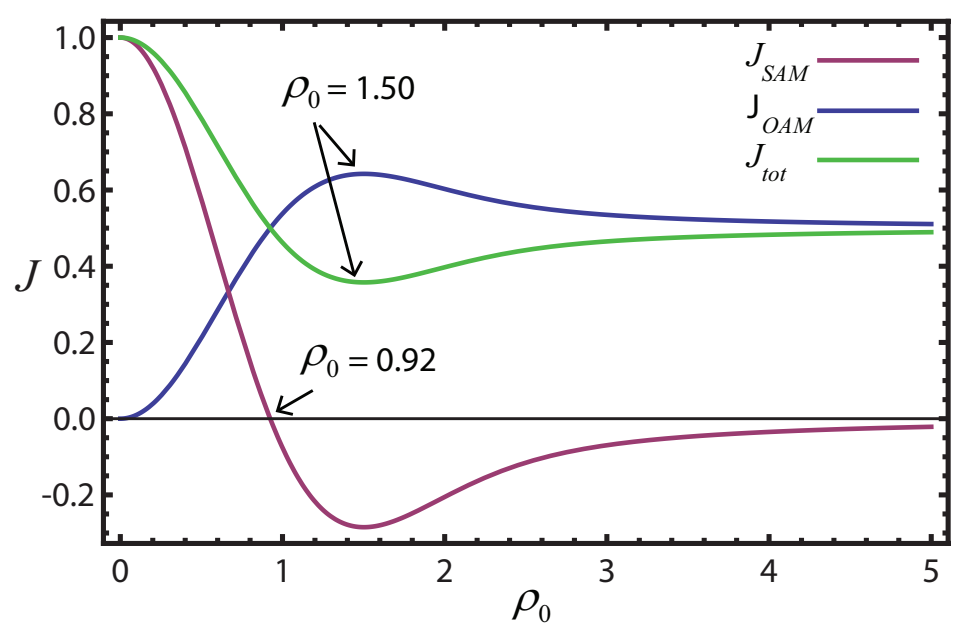

Figure 4.9: Plots of the orbital angular momentum (OAM) (blue line), spin angular momentum (SAM) (purple line) and total angular momentum (green line) of the CR beam as a function of $\rho_{0}$ for a left handed circularly polarized Gaussian input beam carrying a SAM of $+\hbar$. 
Both the value and handedness of the OAM depend on the ellipticity of the input beam and on the $\rho_{0}$ parameter, see Fig. 4.9. For $\rho_{0} \gg 1$ and a CP input beam, i.e., with $J_{0}= \pm \hbar$ ( + for LHCP and - for RHCP), the output CR beam carries total optical angular momentum $\pm \hbar / 2$ and zero SAM. This is natural if one pays attention to the combination of the $B_{0}$ and $B_{1}$ components that give rise to the CR beam. On the one hand, the $B_{0}$ field carries $\pm \hbar \mathrm{SAM}$ and 0 OAM. On the other hand, the $B_{1}$ component carries carries $\mp \hbar \mathrm{SAM}$ and $\pm \hbar$ OAM. Therefore, the equal coherent positive superposition of both fields gives $0 \mathrm{SAM}$ and $\pm \frac{1}{2} \hbar$ OAM.

For values of $\rho_{0}$ out of that condition, the spin-orbit coupling differs. For instance, around $\rho_{0}=0.92$ the handedness of the SAM of the CR beam changes from the same $\left(\rho_{0}<0.92\right)$ to the opposite $\left(\rho_{0}>0.92\right)$ with respect to the input beam. As reported in Ref. [140], for $\rho_{0}=1.50$ the OAM of the CR beam is maximum compared with any other value of $\rho_{0}$. Near the focal plane the twist is more appreciable at the central spot. Additionally, the velocity of rotation of the $S_{1}$ and $S_{2}$ parameters at that region as the beam approaches to the focal plane $(Z=0)$ is much faster than the rotation velocity of the outer ring. For the value $\rho_{0}=0.445$ the beam carries almost null OAM, which explains the small twist of the $S_{1}$ and $S_{2}$ parameters near the focal plane and its similarities with a homogeneously CP Gaussian beam.
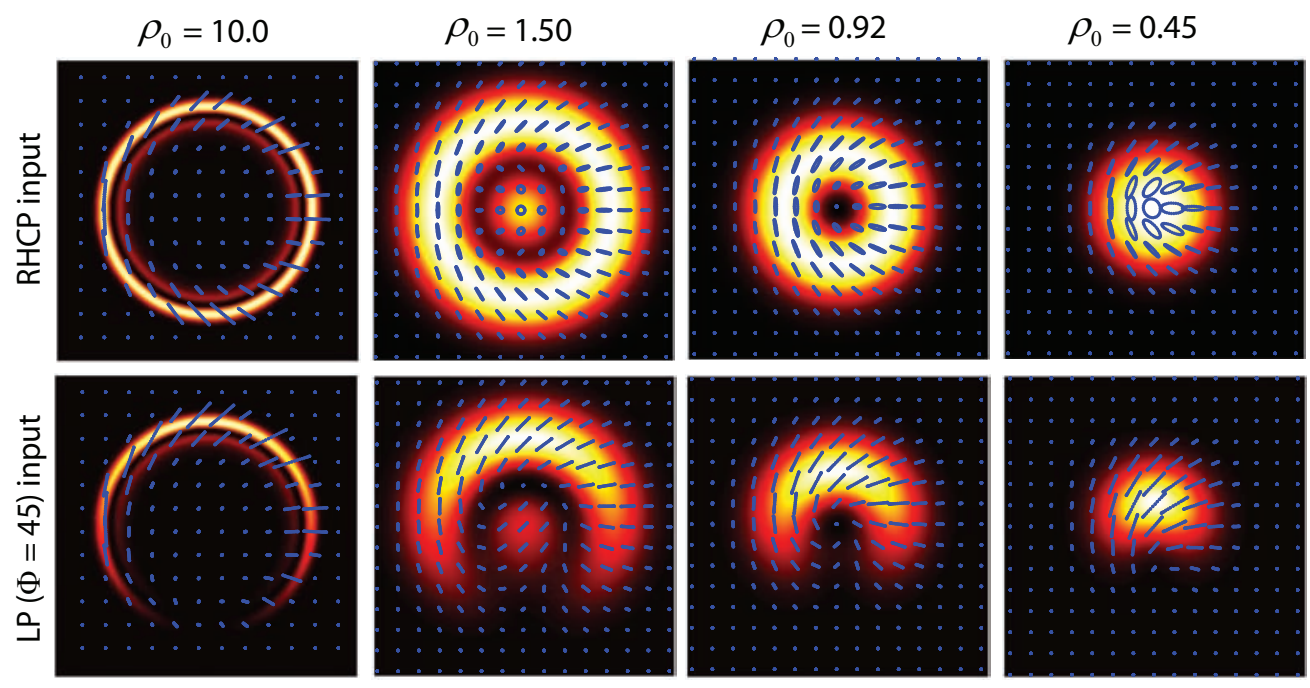

Figure 4.10: Numerically simulated transverse intensity patterns and SOP (blue lines) at $Z=0$ of vector beams obtained for a RHCP (first row) and a $\operatorname{LP}\left(\Phi=45^{\circ}\right)$ input Gaussian beam for $\rho_{0}=10.0$ (first column), $\rho_{0}=1.50$ (second column), $\rho_{0}=0.92$ (third column) and $\rho_{0}=0.45$ (fourth column).

As shown in Fig. 4.3, for $\rho_{0} \lesssim 1$ the intensity patterns of the $B_{0}$ and $B_{1}$ components differ from each other. Since there are regions of the beam where both functions do not coincide, the coherent addition of both functions is not as direct as for $\rho_{0} \gg 1$ and the spin-orbit coupling in the $\rho_{0} \lesssim 1$ case is rather complex. To illustrate some examples, 
note that in some regions of the beam, the amplitude of the electric field vector of the $B_{0}$ and $B_{1}$ components are equal but they have opposite senses. As a consequence, in these regions the total electric field is null and a polarization singularity is found, such as for the case of $\rho_{0}=0.92$. In an alternative scenario, the $B_{0}$ and $B_{1}$ fields have the precise amplitude to generate a region of linearly polarized states, such as the L-line found for $\rho_{0}=1.50$. As a summary, the combination of both SAM and OAM of the CR vector beams leads to the rotation and twisting of the $S_{1}$ and $S_{2}$ Stokes parameters when $\mathrm{CP}$ input beams propagating through the biaxial crystal are considered.

To sum up the the results discussed in this Chapter, Fig. 4.10 presents the calculated transverse patterns and SOP (blue curves) of the vector beams analyzed in this work for a RHCP (first row) and a LP $\left(\Phi=45^{\circ}\right.$ ) (second row) input Gaussian beam at the focal plane. As it can be observed, for low values of $\rho_{0}$ the SOP of the generated vector beams is rich and include regions with different ellipticity and azimuth, leading to Poincare beams [141].

\subsection{Conclusions}

In summary, we have studied in detail the SOP of CR vector beams for a wide range of $\rho_{0}$ and for different SOP of the input beam. We have determined the Stokes parameters of the $\mathrm{CR}$ beam at different transverse planes along the beam propagation direction and we have shown that both the shape and the SOP of the transformed beams depend on the SOP of the input beam. We have shown that the polarization distribution formed by orthogonal polarizations at any two radially opposite points of the pattern and usually associated to the $\mathrm{CR}$ phenomenon remains relevant under the condition $\rho_{0} \gg 1$ and at the focal plane only. For $\rho_{0} \lesssim 1$ and CP input beams we have found that polarization structure of $\mathrm{CR}$ beams contains non-homogeneously elliptically polarized states not only with different azimuth but also with different ellipticity. In contrast, for LP input beams, the SOP of the CR has been reported to be completely linear at the focal plane and with variable ellipticity and azimuth out of it. A good agreement between the theoretical predictions and the experimental results has been obtained.

Additionally, we have shown for the first time to our knowledge, experimental results on polarization singularities of CR beams and we have related them to the spin-orbit coupling provided by the $\mathrm{CR}$ phenomenon. We have demonstrated that CR polarization singularities can be changed by and controlled by varying the SOP of the input light beam. Such polarization singularities as C-points, L-lines and L-circles have been identified for CR beams.

The reported results can be particularly interesting for experiments with tightly focused beams [101], for the generation of novel polarizations in CR [81], in optical micromanipulation [80,142, 143], mode conversion between Heremite-Gauss-like beams and Laguerre-Gauss-like beams [76] and in super-resolution imaging [85]. It is also promising the generation of polarization-tunable potentials to inject, extract and ma- 
nipulate ultra-cold atoms [55, 144]. Additionally, by taking into account that the CR beams posses orbital angular momentum [140] and that the biaxial crystals used are transparent to a wide frequency range, the presented technique could be an alternative for the production of high-frequency vortex beams for molecular-scale super-resolution microscopy [145]. Finally, by using quantum sources, CR vector beams can be a tool for testing new Bell-like inequalities with hybrid polarization-momentum states useful in quantum information technologies [146]. 



\section{CHAPTER 5}

\section{Conical refraction healing after partially blocking the input beam}

In this Chapter we address the question of the CR healing of a Gaussian input beam in the presence of an obstruction. In our case, we investigate the reconstruction of the transverse intensity pattern of the CR beams at different propagation distances and also of their state of polarization when the Gaussian input beam passing through the biaxial crystal is blocked by an azimuthal obstruction. We show that, even for relatively large obstacles, the CR beams keep their annular shape, state of polarization and dark optical singularities. The Chapter is organized as follows: in Sec. 5.1 we introduce related works to the field of self-healing that can be found in the literature and motivate our work. Then, Section 5.2 is devoted to describe our experimental set-up and report healing of conically refracted Gaussian beams after an obstruction for $\rho_{0} \gg 1$. In Section 5.3 we compare the reconstructing behavior of the CR beam for $\rho_{0}=0.92$ and $\rho_{0} \gg 1$ and discuss the differences between the two cases. Finally, our main results are summarized in Section 5.4.

The research contained in this Chapter has been done in collaboration of Ramón Corbalán from the Universitat Autònoma de Barcelona and has been published in Ref. [147].

\subsection{Introduction}

Gaussian beams are the most well known solution of the paraxial wave equation. They are form-invariant beams, i.e., the form of their transverse intensity pattern does not change upon propagation, apart from a scaling factor. Durnin et al. [148] reported another solution of the paraxial wave equation, the Bessel beams, which are completely invariant upon propagation. In other words, both the transverse intensity profile and scale of Bessel beams remain unchanged as it propagates, i.e., Bessel beams 
are diffraction-free beams. One of the main features of Bessel beams is that they selfreconstruct after an obstacle, being this effect known as self-healing.

Recently, there has been a great interest in the study of the self-healing effect appearing in Bessel beams [149, 150] and other diffraction-free beams including Airy beams [151,152] and Pearcey beams [153], or other exotic beams such as helico-conical beams [154] as well as Mathieu and Webber beams [155]. The major advantage of self-healing beams is that they can be used through turbulent media [152] and that they are ideal candidates for particle manipulation at different planes [156, 157] and in microscopy [158]. The CR phenomenon has been also reported as an efficient tool to generate Bessel beams 62, 159, 160]. The relation of CR with Bessel beams suggests that even if the input Gaussian beam is partially blocked, the CR beam may only be slightly affected. The aim of this Chapter is precisely to investigate the CR healing of a Gaussian input beam in the presence of an obstruction that blocks an azimuthal sector of the beam. We analyze both theoretically (by using the theoretical formalism presented in Sec. (2.2.2) and experimentally the influence of the obstruction over both the transverse intensity pattern and the state of polarization of the CR beam for well developed CR rings $\left(\rho_{0} \gg 1\right)$ and for the three-dimensional dark focus reported in Sec. 3.2.

\subsection{CR healing of Gaussian beams for $\rho_{0} \gg 1$}

In this Section we analyze the reconstruction of conically refracted Gaussian beams under the condition $R_{0} \gg w_{0}$ when an obstruction of closing angle $\phi$ blocks an azimuthal part of the input beam. We consider first the approximation $R_{0} \gg w_{0}$ since it is the commonly used configuration in most experimental arrangements. Fig. 5.1 shows our experimental set-up. We obtain a circularly polarized Gaussian beam at $640 \mathrm{~nm}$ from a diode laser coupled to a monomode fiber by utilizing a linear polarizer (LP) and a quarter wave-plate (QWP). As an obstruction, we use amplitude angular masks (AAM). They block an azimuthal sector of angle $\phi$ of the Gaussian beam at the exit of the collimator. The AAM are made by printing the desired 2D pattern over a transparent sheet of plastic. The beam, whose waist radius $w_{0}$ can be adjusted by means of the collimator, passes through a biaxial crystal and parallel to one of the optic axes. A CCD camera combined with an imaging lens (IL) records the transverse intensity pattern of the CR beam at different planes. As a biaxial crystal we use a $\mathrm{KGd}\left(\mathrm{WO}_{4}\right)_{2}$ crystal of length $l=28 \mathrm{~mm}$, conicity $\alpha=16.9 \mathrm{mrad}$ and, therefore, $R_{0}=l \alpha \approx 475 \mu \mathrm{m}$. By reducing the focused beam waist down to $w_{0}=44 \mu \mathrm{m}$, we have obtained a $\rho_{0}$ parameter up to 10.75 .

Fig. 5.2 shows both the experimental (top row) and numerically calculated (bottom row) transverse intensity patterns at the focal plane for obstructions of $(\mathrm{a}, \mathrm{f}) \phi=0^{\circ},(\mathrm{b}, \mathrm{g})$ $45^{\circ},(\mathrm{c}, \mathrm{h}) 90^{\circ}$, (d,i) $135^{\circ}$ and $(\mathrm{e}, \mathrm{j}) 180^{\circ}$. Insets represent the obstructed input beam just behind the AMM. When a relatively small obstruction angle is considered $\left(\phi=45^{\circ}\right)$, the transverse intensity pattern is almost unaffected as compared with the case without obstruction. In this case, the Poggendorff dark ring and the two ring-like structures are 
clearly visible. The rings are mirror-symmetric with respect to the horizontal axis but they have a maximum at their top and bottom regions. As $\phi$ increases the $\mathrm{CR}$ rings become asymmetric and the outer ring breaks into two boomerang-like lobes such that no complete Poggendorff dark ring is appreciable. For $\phi=180^{\circ}$, i.e., when the AAM blocks half of the input beam, the CR transverse intensity pattern is formed by a wide single ring with two dark singularities in the upper and top regions of the ring.

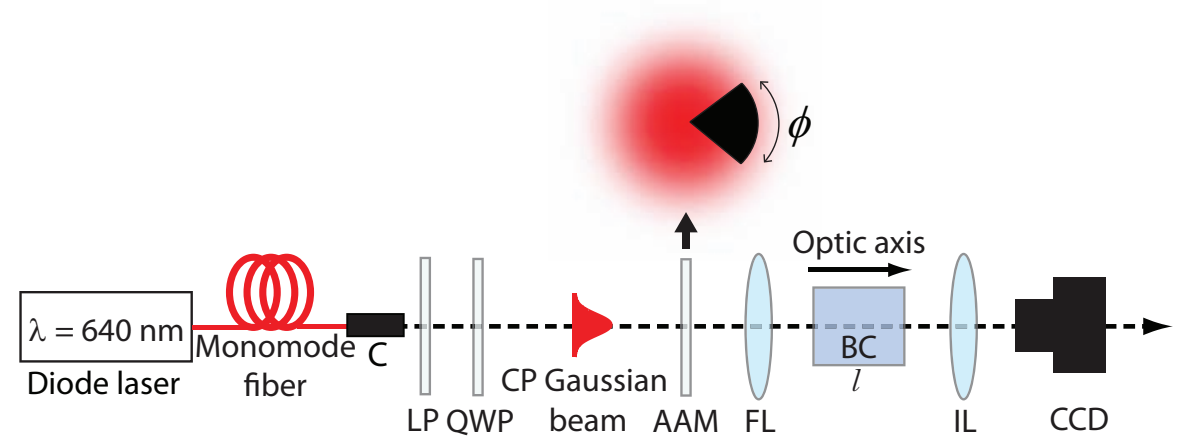

Figure 5.1: Experimental set-up. A Gaussian beam is obtained from a diode laser coupled to a monomode fiber. A collimator $(\mathrm{C})$ is used to change the beam waist radius $w_{0}$ of the Gaussian beam. A linear polarizer (LP) and a quarter wave-plate (QWP) are used to fix the state of polarization of the Gaussian beam to circular. Azimuthal angular amplitude masks (AAM) characterized by their closing angle $\phi$ are used to block a sector of the Gaussian beam. The obtained beam passes through the biaxial crystal (BC) along one of its optic axes and a CCD camera and an imaging lens (IL) record the transverse intensity pattern at different planes.

The healing mechanism provided by the biaxial crystal to reconstruct the CR beam can be understood in terms of the wave-vector splitting within the crystal. Every planewave is described by a certain wave-vector $\mathbf{k}=\mathbf{k}_{\|}+\mathbf{k}_{\perp}$, with $\mathbf{k}_{\perp}=\left|k_{\perp}\right|\left(\cos \phi_{k}, \sin \phi_{k}\right)$. The biaxial crystal splits every plane wave into two new plane-waves. At the focal plane, these two plane-waves are refracted at positions on the ring characterized by their azimuthal angle $\varphi=\phi_{k}$ and $\varphi=\phi_{k}+\pi$ [54. As a consequence, when one azimuthal sector of the Gaussian beam is blocked, the azimuthally opposite sector partially compensates the absence of the blocked sector. For this reason, even when half of the input beam is blocked, a ring-like structure can be formed after passing through the biaxial crystal. This mechanism explains why a single bright ring without Poggendorff splitting is obtained when half of the input beam is blocked with the AAM, i.e., when $\phi=180^{\circ}$. The two bright rings with Poggendorff splitting appear as an interference of plane waves going to a particular azimuthal point of the CR ring from opposite sectors of the input beam. In contrast, if there are no other waves coming to the corresponding opposite points of the $\mathrm{CR}$ ring pattern at the focal plane, there is no interference and a only 
a single ring is observed. In Figs. 5.2(b)-(e) it is clearly visible an increase of the azimuthal sector of a single bright ring and shrink of the double bright rings' domain with the AMM closing angle $\phi$. Note that the azimuthal sector occupied by the double bright rings is larger than the angular sector of the AMM, $\phi$, because of the diffraction of the input beam at the edges of AMM dark sector.

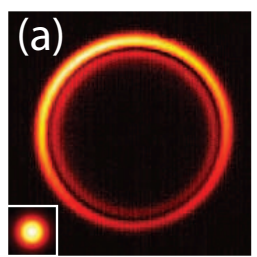

$\phi\left({ }^{\circ}\right)=0$

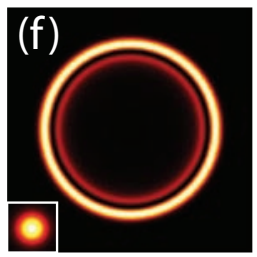

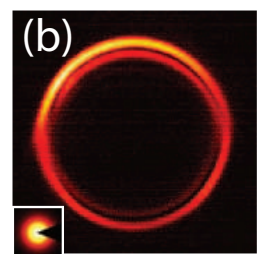

45

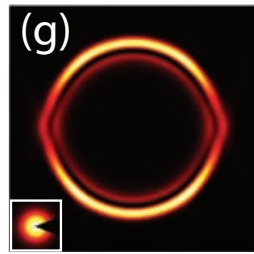

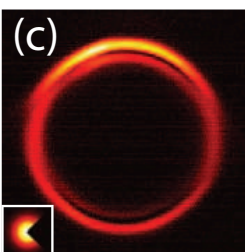

90

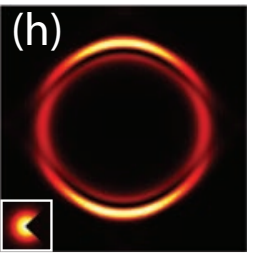

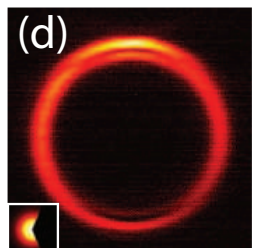

135

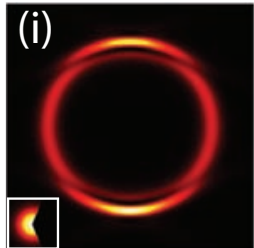

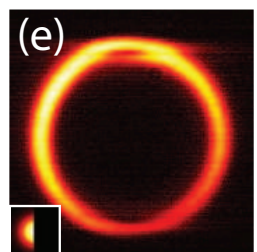

180

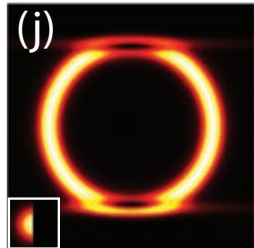

Figure 5.2: Transverse intensity patterns at the focal plane $(z=0)$ obtained with an obstruction of closing angle (a,f) $\phi=0^{\circ},(\mathrm{b}, \mathrm{g}) \phi=45^{\circ},(\mathrm{c}, \mathrm{h}) \phi=90^{\circ},(\mathrm{d}, \mathrm{i}) \phi=135^{\circ}$ and $(\mathrm{e}, \mathrm{j}) \phi=180^{\circ}$ placed before the biaxial crystal. (a,f) Intensity pattern obtained in the absence of obstruction. First row: experimental results $\left(\rho_{0}^{\exp }=10.75\right)$. Second row: numerical calculations obtained from Eqs. (2.3), (2.3), (2.19) $-(2.22)\left(\rho_{0}^{\text {th }}=10\right)$.

Now we turn to analyze the healing of the state of polarization of the reconstructed CR beams. The standard tool to analyze the state of polarization of a light beam is the Stokes vector $S=\left(S_{0}, S_{1}, S_{2}, S_{3}\right)$ together with the azimuth $\epsilon$ and ellipticity $\beta$ of the polarization ellipse of the light beam described by Eqs. (4.11)-(4.16). The values of $\epsilon$ and $\beta$ at the focal plane numerically calculated for obstructions of $(\mathrm{a}, \mathrm{f}) \phi=0^{\circ},(\mathrm{b}, \mathrm{g})$ $45^{\circ},(\mathrm{c}, \mathrm{h}) 90^{\circ},(\mathrm{d}, \mathrm{i}) 135^{\circ}$ and $(\mathrm{e}, \mathrm{j}) 180^{\circ}$ are presented in Fig. 5.3. Insets represent the obstructed input beam. Ideally, as $\rho_{0} \rightarrow \infty, \beta \rightarrow 0$. Since in our numerical simulations we consider $\rho_{0}=10$, the state of polarization of the CR rings is slightly elliptical rather than purely linear. As it can be appreciated, in general, the polarization structure of the CR beam is maintained for all the values of $\phi$, i.e., every two diametrically opposite points of the light structure are orthogonally polarized.

In Fig. 5.4 we show the evolution of the transverse intensity patterns along the axial direction for a blocking sector of $\phi=45^{\circ}$. Top row shows the transverse intensity patterns in the absence of the blocking mask, while middle and bottom rows are the experimental and numerically calculated transverse intensity patterns for the obstructed Gaussian beam. Near the focal plane $(z=0)$ the transverse intensity pattern resembles the pattern obtained without obstruction. In contrast, far enough of the focal plane it can be appreciated a perturbation of the CR transverse intensity pattern that resembles 
the considered obstruction.

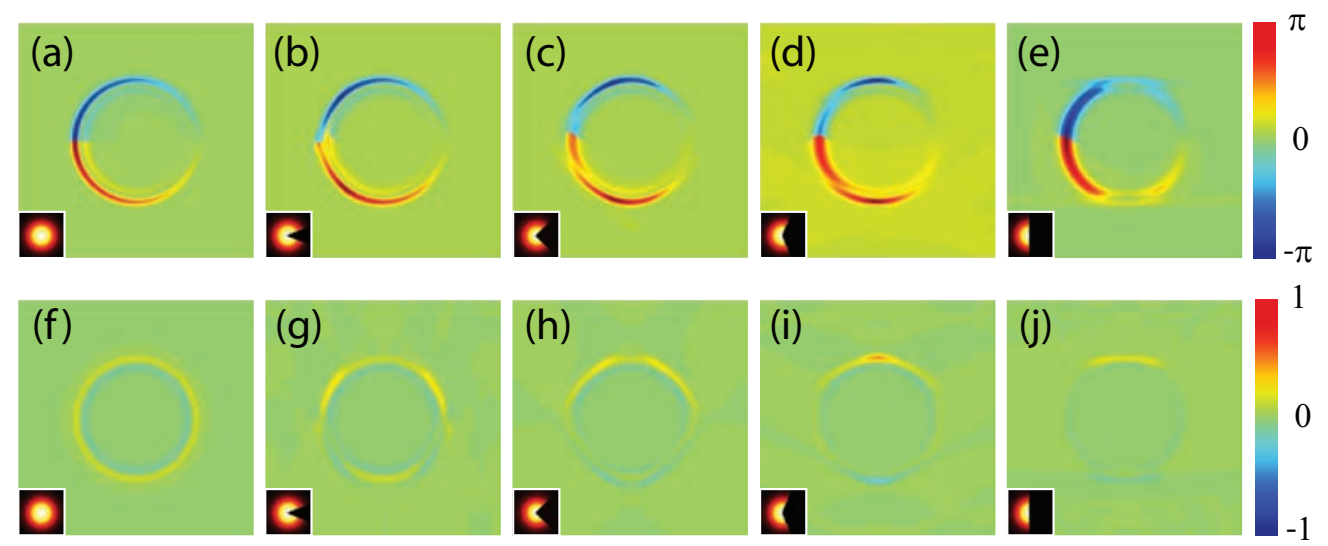

Figure 5.3: Numerically calculated 2D density plots of the azimuth $\epsilon$ (first row) and the ellipticity $\beta$ (second row) of conically refracted beams at the focal plane for $\rho_{0}=10$ when the input Gaussian is blocked by azimuthal obstructions of $(\mathrm{b}, \mathrm{g}) \phi=45^{\circ},(\mathrm{c}, \mathrm{h})$ $\phi=90^{\circ},(\mathrm{d}, \mathrm{i}) \phi=135^{\circ}$ and $(\mathrm{e}, \mathrm{j}) \phi=180^{\circ}$ is placed before the biaxial crystal. (a,f) $2 \mathrm{D}$ density plots of $\epsilon$ and $\beta$ in the absence of the obstruction.
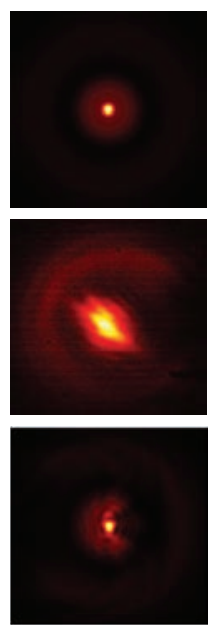

$Z=-15$
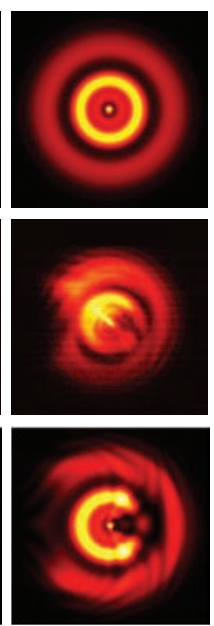

$-10$
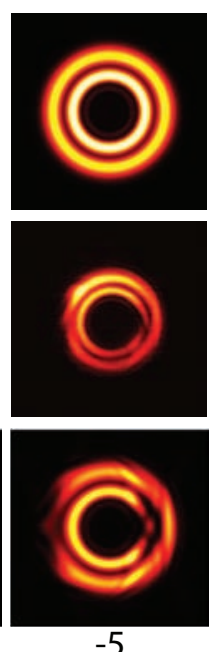

$-5$
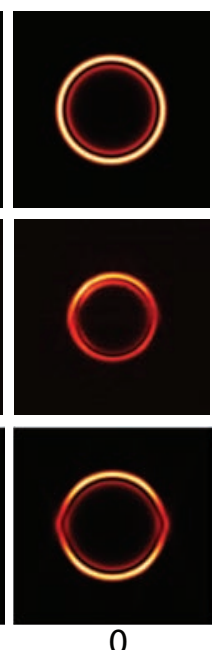
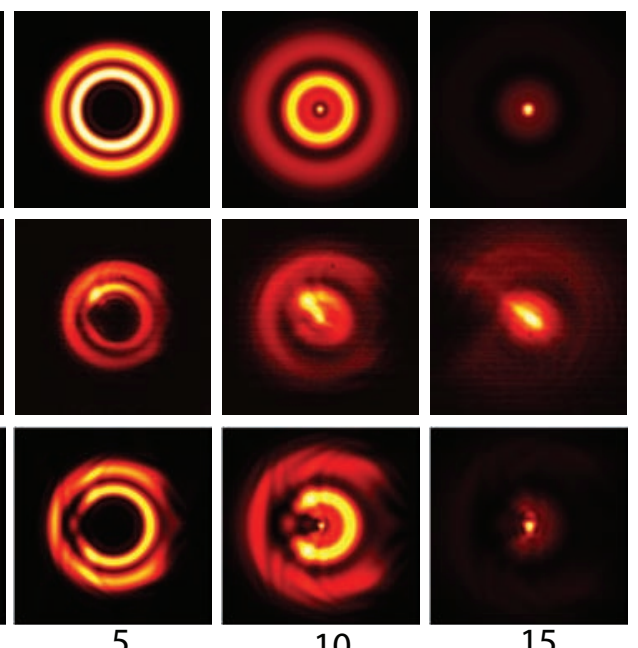

10
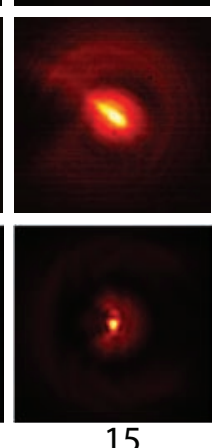

Figure 5.4: Experimental (second row) and numerically calculated with Eqs. (2.3), (2.3), (2.19) -(2.22) (third row) transverse intensity patterns along the axial direction $z$ for an obstruction with $\phi=45$ for $\rho_{0}=10$. The first row shows the numerically calculated transverse intensity patterns obtained in the absence of the obstruction. $z$ is measured in units of the Rayleigh range, which for the Gaussian beam used in our experiments $\left(w_{0}=44 \mu \mathrm{m}\right)$ is $z_{R}=9.5 \mathrm{~mm}$. 


\subsection{CR healing of Gaussian beams for $\rho_{0} \approx 1$}

As discussed in Section 2.4.1, the CR beam depends strongly on the value of the control parameter $\rho_{0}$. For $\rho_{0}=0.92$ the transverse intensity pattern at the focal plane forms a doughnut-like light structure with a null intensity point at the beam center [66], see Fig. 5.5(a). Along the axial direction, the intensity at the beam center is no longer zero and the beam forms an optical bottle. This value of $\rho_{0}$ is particularly interesting because the polarization distribution of the light ring has points with different $\beta$ and $\epsilon$ and one deals with a Poincare beam, i.e., a beam possessing points with all the polarization states of the Poincare sphere [141]. In what follows we discuss the reconstruction of a conically refracted Gaussian beam for $\rho_{0}=0.92$ after an obstruction, analogously to what has been performed previously for $\rho_{0} \gg 1$. For the incoming experiments we have used the same set-up as in Fig. 5.1 but with a $2.3 \mathrm{~mm}$ long $\mathrm{KGd}\left(\mathrm{WO}_{4}\right)_{2}$ (therefore $\left.R_{0}=39 \mu \mathrm{m}\right)$ crystal and a waist radius of $w_{0}=44 \mu \mathrm{m}$.
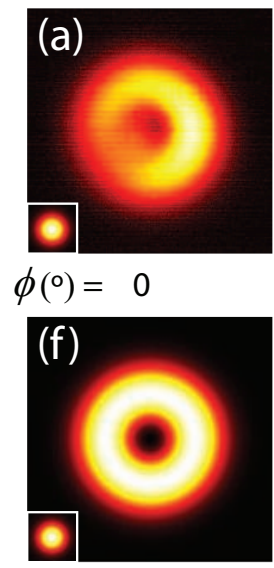

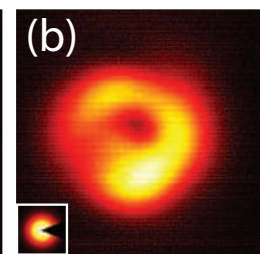

45

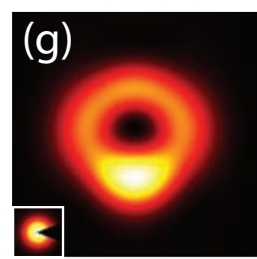

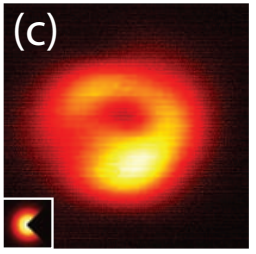

90

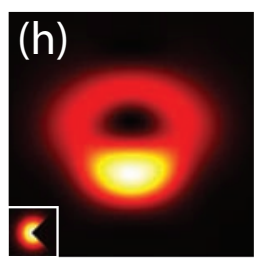

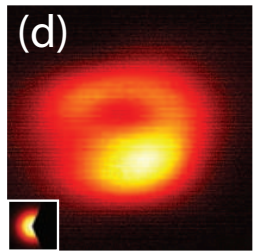

135

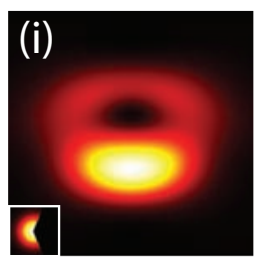

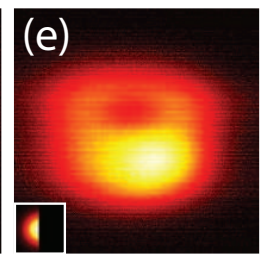

180

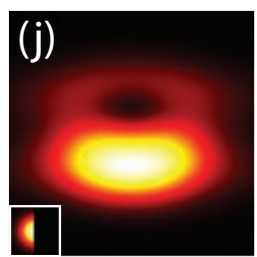

Figure 5.5: Transverse intensity patterns at the focal plane $(z=0)$ obtained when an obstruction of angle $(\mathrm{b}, \mathrm{g}) \phi=45^{\circ},(\mathrm{c}, \mathrm{h}) \phi=90^{\circ},(\mathrm{d}, \mathrm{i}) \phi=135^{\circ}$ and $(\mathrm{e}, \mathrm{j}) \phi=180^{\circ}$ is placed before the biaxial crystal. (a,f) Transverse intensity pattern in the absence of obstruction. First row: experimental results $\left(\rho_{0}^{\exp }=1.04\right)$. Second row: numerical calculations obtained from Eqs. (2.3), (2.3), (2.19) $-(2.22)\left(\rho_{0}^{\text {th }}=0.92\right)$.

Fig. 5.5 shows both the experimental (top row) and numerically calculated (bottom row) transverse intensity pattern at the focal plane for obstructions of $(\mathrm{a}, \mathrm{f}) \phi=0^{\circ},(\mathrm{b}, \mathrm{g})$ $45^{\circ},(\mathrm{c}, \mathrm{h}) 90^{\circ},(\mathrm{d}, \mathrm{i}) 135^{\circ}$ and $(\mathrm{e}, \mathrm{j}) 180^{\circ}$. Insets represent the obstructed input beam for $\rho_{0}=0.92$. In contrast to the case of $\rho_{0} \gg 1$, when a relatively small obstruction angle is considered $\left(\phi=45^{\circ}\right)$, the transverse intensity pattern is substantially different with respect to the case with no obstruction. In this case, a maximum of intensity appears at the bottom part of the ring and, therefore, the intensity pattern is mirror symmetric with respect to the vertical axis. As $\phi$ increases, the intensity in the bottom part of the light structure becomes stronger than in the top part. For all the values of $\phi$, an 
intensity minimum can be observed but its position moves in the vertical direction as $\phi$ increases. However, note that even when half of the beam is blocked by the obstruction, the dark singularity is preserved. A similar behavior has been reported for a linearly polarized Bessel beam [150].

With respect to the state of polarization of the CR beams at the focal plane, see Fig. 5.6, we have observed that there is a tendency to preserve the polarization structure of the CR beam without obstruction: the CR beam is elliptically polarized and the ellipticity changes radially similarly to the intensity pattern. At the edges of the beam, $\beta \rightarrow 0$ and the characteristic CR polarization distribution is recovered. However, since the transverse intensity pattern is very affected by the presence of an obstruction, these features of the state of polarization are lost for large enough values of $\phi$. In particular, $\beta$ losses its doughnut-like shape, while $\epsilon$ is kept quite stable up to $\phi=135^{\circ}$.

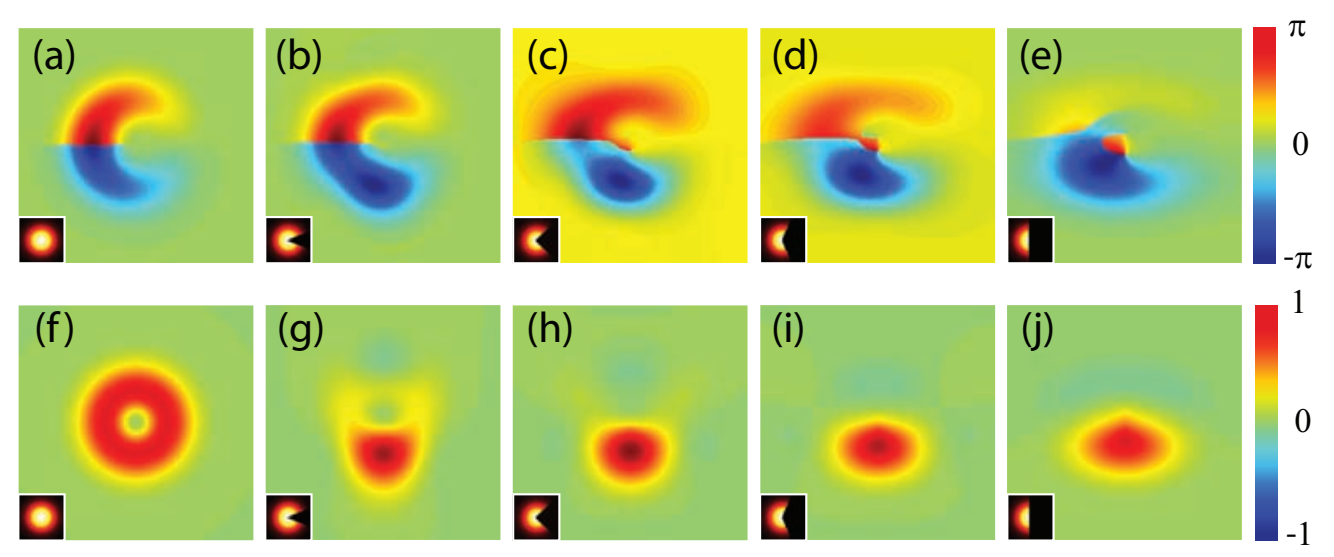

Figure 5.6: 2D density plots of the azimuth $\epsilon$ (first row) and the ellipticity $\beta$ (second row) of conically refracted beams at the focal plane for $\rho_{0}=0.92$ when the input Gaussian is blocked by azimuthal obstructions of (b,g) $\phi=45^{\circ},(\mathrm{c}, \mathrm{h}) \phi=90^{\circ},(\mathrm{d}, \mathrm{i}) \phi=135^{\circ}$ and $(\mathrm{e}, \mathrm{j}) \phi=180^{\circ}$ is placed before the biaxial crystal. (a,f) $2 \mathrm{D}$ density plots of $\epsilon \beta$ in the absence of obstruction.

The evolution of the transverse intensity pattern along the axial direction for a blocking sector of $\phi=45^{\circ}$ is shown in Fig. 5.7. Top row shows the transverse intensity patterns in the absence of the blocking mask, while middle and bottom row are the experimental and numerically calculated transverse intensity patterns for the obstructed Gaussian beam. Near the focal plane $(z=0)$ the transverse intensity pattern differs from the one obtained without obstruction. Additionally, out of the focal plane there is no reconstruction of the transverse intensity pattern. Therefore, for $\rho_{0}=0.92$, the beam reconstruction process is neither found at the focal plane nor away from it. 

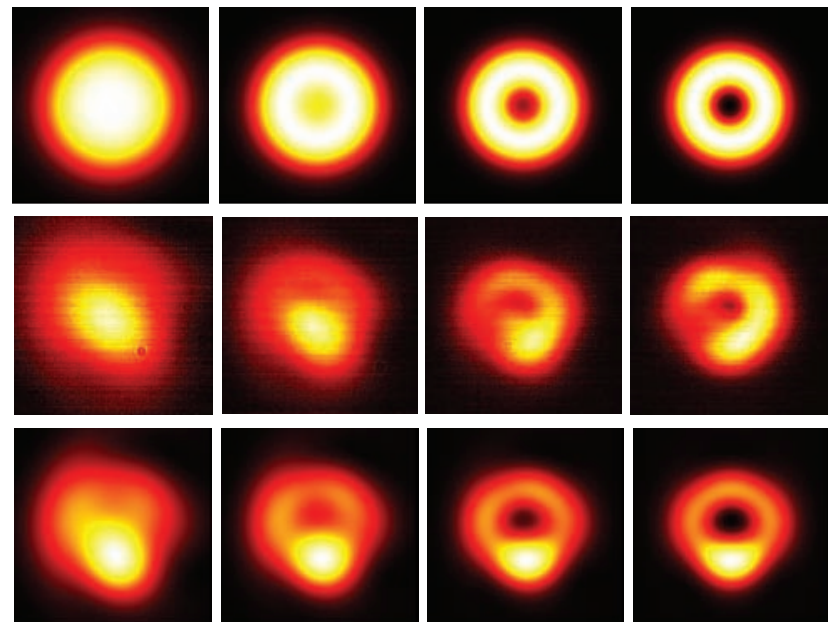

$z=-1.5$

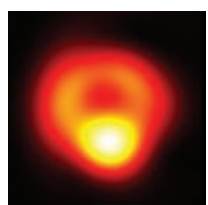

$-1.0$
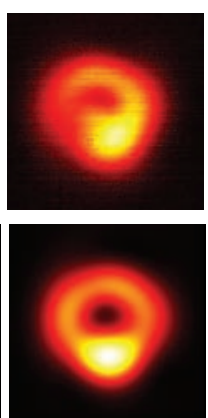

$-0.5$
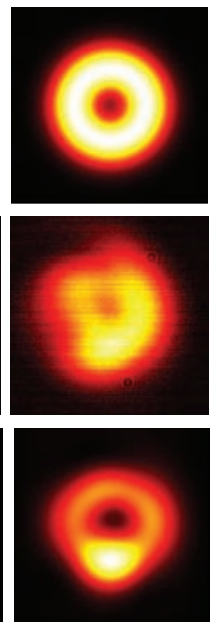

0.5
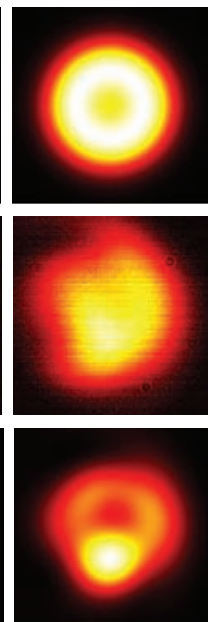

1.0
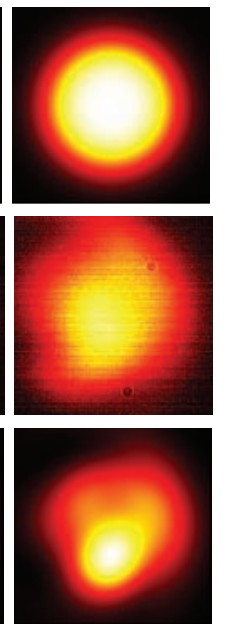

1.5

Figure 5.7: Experimental (second row) and numerically calculated (third row) with Eqs. (2.3), (2.3), (2.19)-(2.22) transverse intensity patterns along the axial direction $z$ for an obstruction with $\phi=45$ for $\rho_{0}=0.92$. The first row shows the numerically calculated transverse intensity patterns obtained in the absence of the obstruction. $z$ is measured in units of the Rayleigh range, which for the Gaussian beam used in our experiments $\left(w_{0}=44 \mu \mathrm{m}\right)$ is $z_{R}=9.5 \mathrm{~mm}$.

\subsection{Conclusions}

We have analyzed in detail the transformation of Gaussian beams partially obstructed when they propagate through a biaxial crystal and parallel to one of the optic axes, i.e., under conditions of CR. We have shown that, at the focal plane, the CR beams for $\rho_{0} \gg 1$ preserve the annular shape even when half of the beam is blocked. However, we have found that the dark annular singularity known as Poggendorff dark ring only remains for small perturbations of the input beam. Out of the focal plane we have obtained that the obstruction affects the beam evolution, being its effect more appreciable the further away one moves along the axial direction. Additionally, the reconstruction of the state of polarization of the CR beam has also been investigated. For $\rho_{0} \gg 1$, we have found that the polarization distribution of the CR rings is very stable against large perturbations.

We have carried out analogous investigations for $\rho_{0} \approx 1$. In this case the transverse light pattern is more affected by the presence of the obstruction than in the case of $\rho_{0} \gg 1$. Regarding the reconstruction of the state of polarization we have found that only the azimuth of the polarization is relatively robust when large obstructions affect the input Gaussian beam. 


\section{CHAPTER 6}

\section{An optical vault for absorbing particles}

This Chapter is devoted to demonstrate that the CR bottle beam obtained for $\rho_{0}=R_{0} / w_{0} \gg 1$ can be used as a reconfigurable optical potential to trap absorbing particles throughout the photophoretic force. We take profit of the characteristic state of polarization of the CR bright rings to generate a null-intensity region at the top region of the ring that allows particles to enter into the optical trap. Then, by changing the state of polarization of the input beam from linear to circular, we close the bottle and confine particles. Finally, by modifying again the state of polarization of the input from circular to linear beam we generate a null-intensity region at the bottom of the ring and the trapped particles can scape from the trap in a controlled manner. The Chapter is organized as follows. In Sec. 6.1 we motivate our approach and discuss related works available in the literature. Then, Sec. 6.2 presents the fundamentals on the photophoretic force. Our experimental proposal, which only consists on the use of half- and quarter-waveplates, a focusing lens and a biaxial crystal in addition to the input laser beam is shown in Sec.6.3. In Sec.6.4, we demonstrate experimental efficient loading and unloading of carbon-coated glass shells with sizes ranging from $20 \mu \mathrm{m}$ to $50 \mu \mathrm{m}$. Finally, the conclusions of our work are shown in Sec. 6.5

The research contained in this chapter has been published in Refs. 142, 161, and has been done in collaboration with Vladlen Shvedov, Cyril Hnatovsky and Wieslaw Krolikowski from the Australian National University.

\subsection{Introduction}

Since its inception in the late 70s the field of optical trapping and manipulation of micron and submicron-sized objects with light has experienced an intense interest 
and rapid development [162,163]. Optical tweezers utilizing the presence of mechanical forces arising from light interaction with matter are now an indispensable tool in various physical, biological and medical applications. They have been extensively used on manipulating colloidal particles, molecules, nanoparticles and even single atoms. Last decade has seen an enormous progress in the field of trapping resulting in the implementation of advanced techniques involving for instance, multiple holographic traps, optical fibers, or singular scalar and vector beams [164, 165. Optimum conditions for particle trapping are dictated by the optical properties of the particles and the surrounding medium, as well as the physical nature of the light-mediated trapping forces. For instance, while high light intensity attracts and traps transparent high-index objects in a low-index medium, it in fact repels low index particles in a high index environment [166]. That is why hollow (or doughnut) beams are used for efficient trapping in the latter case. In general, depending on the particular media and application, robust trapping and manipulation of micro-objects requires tailoring the light beam intensity pattern via phase and amplitude modulation or by varying the spatial coherence of light [165, 167-169].

In 2000, Arlt and Padgett [95] introduced the concept of an optical bottle which represents an optical beam with a low (ideally null) intensity region surrounded entirely by light. Such a bottle could be used as a three-dimensional trap. Following this idea various practical implementations of optical bottles have been proposed. The low intensity regions have been formed using, for instance, interference of multiple laser beams, partially spatially coherent optical vortices or laser beams affected by optical aberrations. The suitability of an optical bottle for particle trapping and manipulation has been confirmed in experiments with atoms [170, 171] and absorbing particles [86, 143, 172, 173.

The problem with an ideal bottle beam is that the more efficiently it traps particles the more difficult it is to load it with particles. Once an optical bottle is formed it actually prevents particles from entering it. To cope with this issue, one straightforward solution consists of turning on the bottle beam when the particles already float in the region where the trap will be formed. Another and much more convenient choice would be to design a bottle in such a way that it could be partially opened and closed so it could be loaded and unloaded with particles as required.

The purpose of this Chapter is to prove that such a design is indeed possible. We demonstrate that optical bottle formed CR can be tailored so that it can be opened and closed at will and in real time by varying the polarization of the input beam. We then use photophoretic trapping to demonstrate loading and unloading of airborne particles into and from the bottle.

\subsection{The photophoretic force}

The photophoretic force is a thermal force induced by optical beams that was identified firstly by Felix Ehrenhaft at the beginning of the $20^{\text {th }}$ century [174]. The force 
of photophoresis works as follows. When a gas-suspended particle is illuminated, light is diffracted, refracted and reflected by the particle. Additionally, if the particle is not transparent, it will absorb part of the energy of the light field and its temperature will rise up. As a consequence, the illuminated particle will radiate heat to the environment and the molecules of the surrounding gas will increase their kinetic energy. If the particle is non-symmetrically illuminated, heat will be radiated unevenly too and only molecules from a certain region will increase their kinetic energy. Those ones will exchange linear momentum with the illuminated particle with the result of a net force applied over the particle. At this point, the movement of the illuminated particle can be away from the light source or towards to it depending on its absorption properties.

(a)

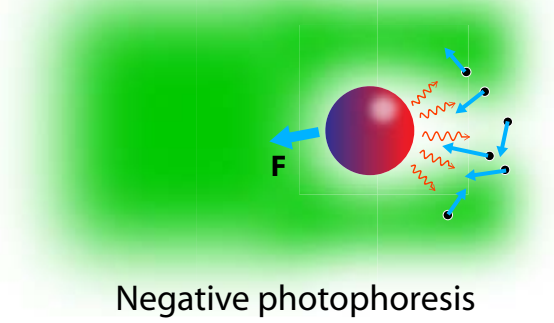

(b)

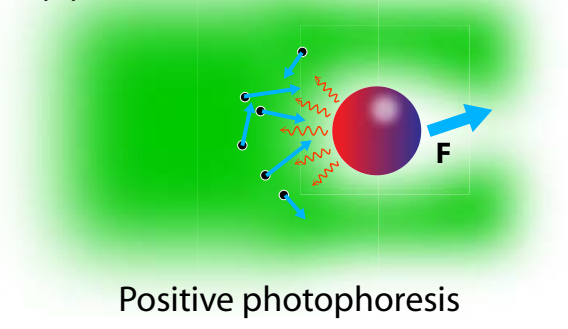

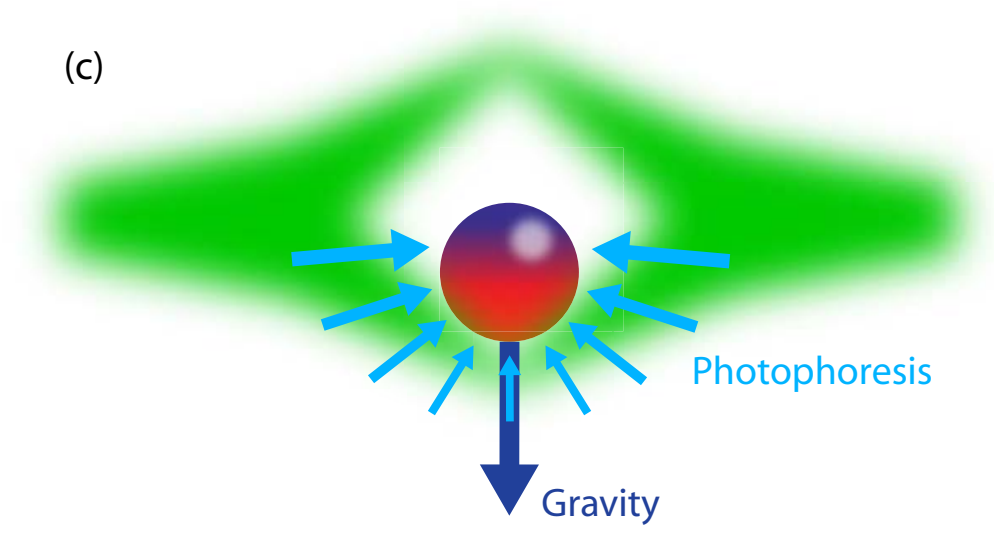

Figure 6.1: Action of the photophoretic force over an absorbing particle. (a) If heat is concentrated at the dark side of the particle, the particles from the surrounding medium will push it toward the light source (negative photophoresis). (b) Positive photophoresis occurs when the illuminated side of the particle is hotter than the dark one. In this case the net motion of the particle is away from the source. (c) If the particle is illuminated symmetrically it can be trapped in a three-dimensional region of space.

First, let's consider the case of a low absorbing spherical particle. In this case, most of the illuminating light is refracted and concentrated at the dark side of the particle. As a consequence, this side will be hotter than the illuminated one and the particle will move towards the light source. This effect is known as negative photophoresis. Negative photophoresis can also occur if heat absorption over the particle's surface is non-uniform, 
as sketched in Fig. 6.1(a). In contrast, if the particle is strongly absorbing, most of the illuminated light will be absorbed at the illuminated side of the particle and its interaction with the surrounding medium will result on a net force away from the source, being this case named as positive photophoresis, see Fig. 6.1(b). In addition, if heat transmission over the particle surface is non-homogeneous, the photophoretic force will be even more involved. In our work, we consider homogeneous carbon-coated spherical glass shells, so that most of particles suffer from positive photophoresis. For further reading on the photophoretic force, we recommend Refs. [172, 174]. The photophoretic force has been used as a speckle trap [169], to trap biological samples [175, 176], combined with engineered vortex beams as a tractor beam [86, 143] and demonstrated to also depend on the polarization of the illuminating source [143, 177].
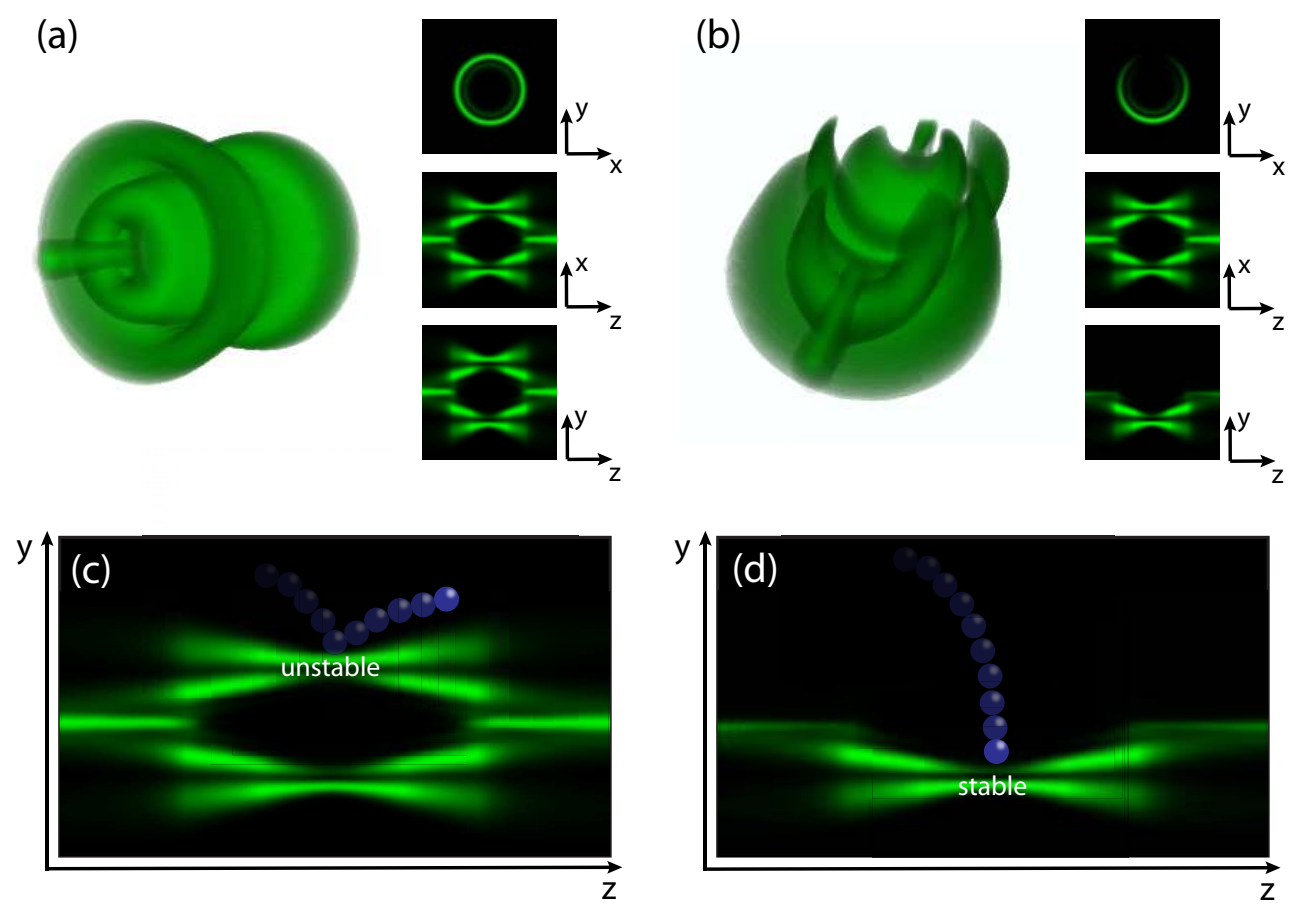

Figure 6.2: Numerically calculated CR optical bottle beams obtained for (a) a circularly polarized input beam and (b) a linearly polarized input beam by using Eqs. (2.14)(2.16). The bottle is fully closed for a circularly polarized incident beam and opened for a linearly polarized incident beam. The hole in the top wall of the "bottle" is clearly visible. The insets depict the principal cross sections of the light intensity distribution. (c) and (d) depict the loading of particles into the bottles. Here $\rho_{0}=10$.

\subsection{Experimental proposal}

Our trapping scheme, which is based on the possibility of opening and closing in real time the optical bottle provided by the CR phenomenon when $\rho_{0} \equiv R_{0} / w_{0} \gg 1$, works as 
follows. Let's consider the case of the CR optical bottle generated when the input beam passing through the biaxial crystal is circularly polarized, see Fig. 6.2(a). In this case the optical bottle will be completely closed in 3D and absorbing particles falling down inside the bottle will be repelled from the latter as sketched in Fig. 6.2(c). Obviously, there is a certain probability for the particles to pass through the light barrier and enter inside the bottle but such probability is very low. In contrast, a linearly polarized input beam impinging the crystal results in the loss of perfect cylindrical symmetry of the CR optical bottle. From the 3D point of view, this latter case results in the formation of a hole in the side of the otherwise perfect bottle beam, see Fig. 6.2(b).

The angular position of null-intensity region can be varied by rotating the azimuth of the linear polarization of the input beam. If the latter coincides with the azimuth from the bottom part of the CR rings at the focal plane, a null intensity point at their top will be generated. This would allow increasing the trapping efficiency, since absorbing particles will find an stable point at the bottom part of the CR rings, as shown in Fig. 6.2(c) and Fig. 6.2(d). Once a particle has been loaded inside the bottle, the latter can be closed by modifying the state of polarization of the input beam from linear to circular, which isolates the particle from the environment. Finally, reverting the state of polarization of the input beam to linear would enable opening a hole in the trap at a desired angular location to unload the particles.

We tested experimentally the practical suitability of the above described optical bottle beams to trap of airborne microscopic particles. Our experimental proposal is rather simple, since only a half-waveplate (HWP), a quarter-waveplate (QWP) a focusing lens and a biaxial crystal is needed to generate the 3D trapping potential, see Fig. 6.3(a). The light beam from a CW laser $(\lambda=532 \mathrm{~nm}$, input power $100 \mathrm{~mW})$ passes through a HWP and QWP and then, after focusing with $100 \mathrm{~cm}$ positive lens, propagates along the optical axis of a monoclinic KTP crystal $(l=10 \mathrm{~mm}$ and $\alpha=10 \mathrm{mrad})$ cut perpendicular to one of its optic axes, giving $\rho_{0} \approx 12$. Light emerging from the crystal is imaged with a CCD camera. We start with a circularly polarized input beam to create a perfect, cylindrically symmetric optical bottle. In order to visualize the optical bottle the camera was translated axially with a $10 \mu \mathrm{m}$ step and at each step the transverse light intensity distribution is recorded and stored in the computer. A sequence of 75 intensity slices is then used to reconstruct the full 3D structure of the bottle. The result is depicted in Fig. 6.3(b). As expected, the CR beam forms an optical bottle with a dark central region entirely surrounded by light. The transverse size and the length of the bottle could be adjusted by varying the collimating optics as well as the position of the crystal. Then, the mutual orientation of the fast axis between the HWP and the QWP is modified to transform continuously the ellipticity of the input beam from $90^{\circ}$ (circularly polarized) to $0^{\circ}$ (linearly polarized). The 3D light intensity distribution for the linearly polarized case is shown in Fig. 6.3(c). The light structure is no longer cylindrically symmetric, with the top wall of the bottle featuring an opening, in agreement with the theoretical prediction, see Fig. 6.2(b). 
(a)

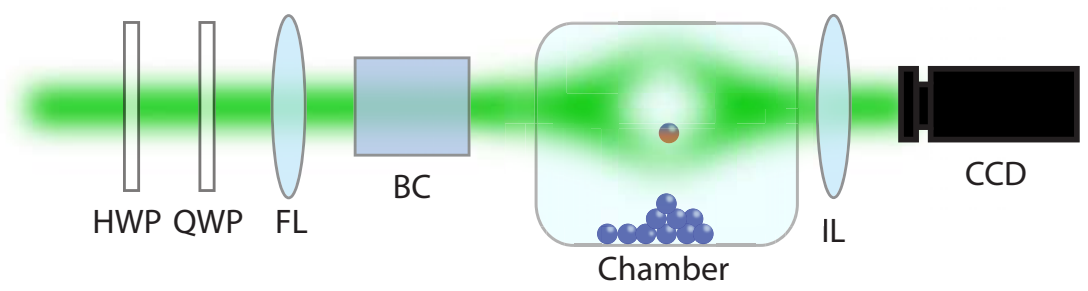

(b)

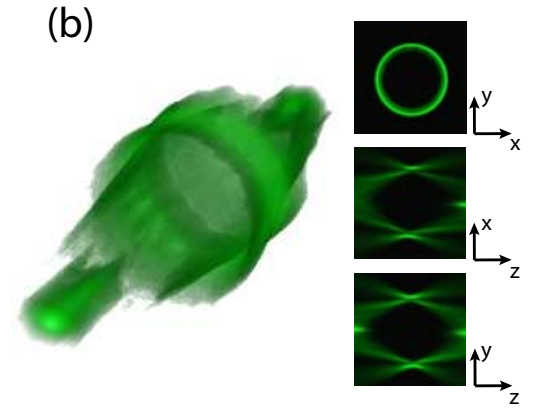

(c)

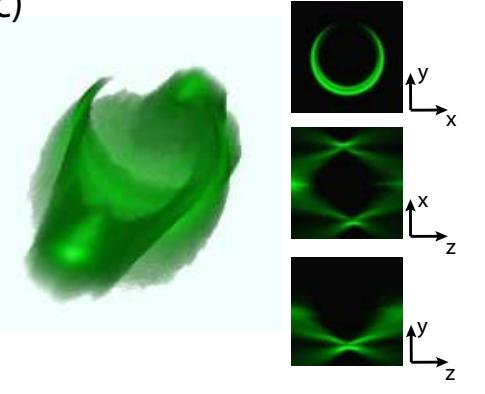

Figure 6.3: (a) Experimental set-up. A Gaussian-like input beam obtained at $532 \mathrm{~nm}$ and with $100 \mathrm{~mW}$ of power is focused by a lens (FL) parallel to one of the optic axis of a KTP biaxial crystal. Previously, the state of polarization of the input beam has been prepared by means of a half-waveplate (HWP) and a quarter-waveplate (QWP). Absorbing particles contained in a chamber are trapped at the generated CR optical bottle. An imaging lens (IL) and a CCD camera are used to image different transverse planes along the beam propagation direciton. (b) and (c) are the reconstructed experimental 3D intensity distribution obtained for circularly polarized and linearly polarized input beams, respectively. Here $\rho_{0}=12$.

\subsection{Experimental results}

We used the experimental set-up and optical bottles depicted in Fig. 6.3 to demonstrate trapping and manipulation of airborne light absorbing particles. Such particles can be efficiently confined by employing the photophoretic force [173, 174]. As commented in Sec. 6.2, in this case the illumination of particles leads to their heating and nonuniform temperature distribution. Interaction with the surrounding air results in the appearance of the photophoretic force which tends to repel particles from the high intensity region. In our experiments with the optical bottle we used glass shells covered with a thin layer of carbon (around $200 \mathrm{~nm}$ thick) in order to enhance light absorption. The external diameter of the shells ranged from a few to tens of micrometers. To prevent accidental air flow from affecting the trapping the optical bottle was formed inside a transparent glass cell placed immediately behind the biaxial crystal. The axially located CCD camera recorded images of the particles inside the optical bottle. In order to speed up the trapping process the spheres were made floating in the air. 
(a)

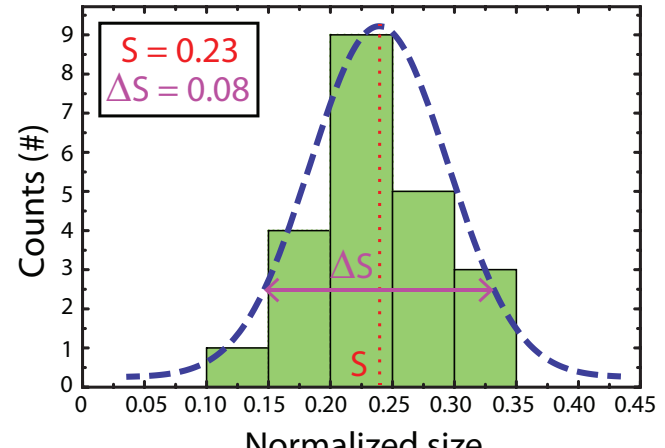

(b)
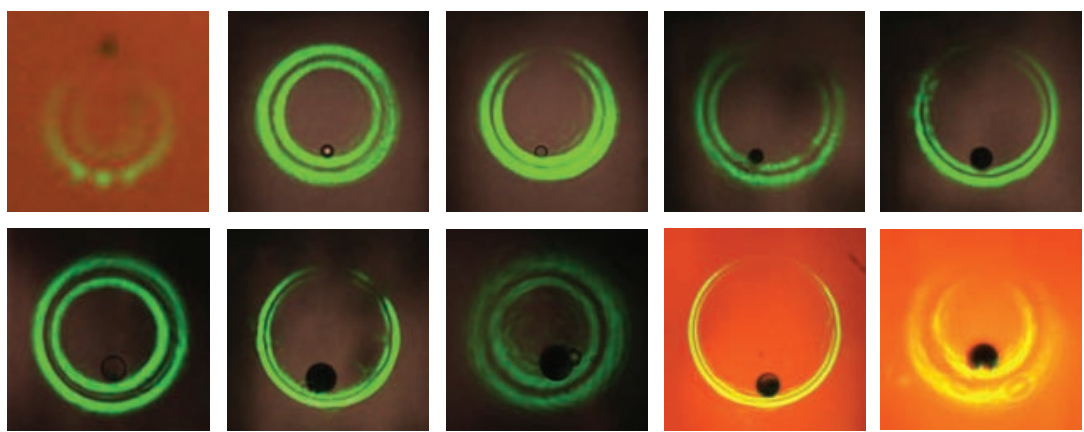

Figure 6.4: (a) Statistics of the particles size normalized to the radial dimensions of the trap. (b) Experimental images of hollow glass shells of different size trapped inside the bottle beam. Particles diameter has been measured to be in the range $20-50 \mu \mathrm{m}$. Note that simultaneous trapping of two glass spheres is also possible. Here $\rho_{0} \approx 12$, being the ring radius $R_{0} \approx 200 \mu \mathrm{m}$.

We found that while a particle could be trapped using either a fully closed (circular polarization) or open (linear polarization) bottle, the loading process was much faster in the latter case. As the internal diameter of the bottle was rather large $(200 \mu \mathrm{m})$ the bottle could accommodate a great variety of trapped spheres. In Fig. 6.4(b) we show examples of particles with different size confined in the trap. Because of gravity they are all located at the bottom of the bottle. The trapping was generally very robust, with the particles resting on the lower "wall", although other stable trapping positions are also possible. However, we found that sometimes the trapped particles oscillated inside the trap with the oscillation frequency increasing with the trapping power. Such dynamics was observed in the case of trapping complex objects such as those formed by two connected glass spheres. Besides, in Fig. 6.4(a) it is shown an histogram with statistics on the particle size normalized to the radial dimensions of the trap, i.e., the diameter of the CR ring. The size distribution is Gaussian-like, with an average normalized size of 0.23. That is to say it is possible to efficiently trap particles of as big as the half of the CR ring radius $R_{0}$. 

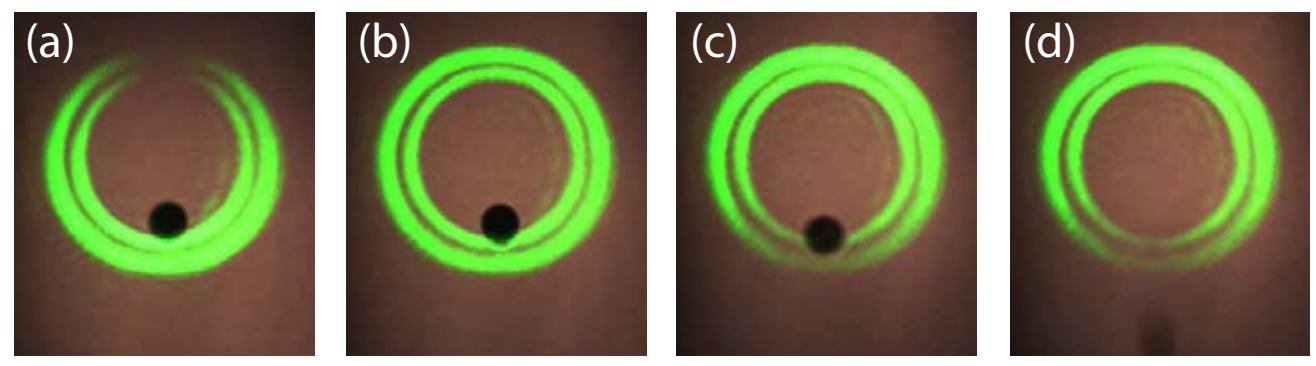

Figure 6.5: Experimentally recorded sequence of images illustrating unloading the trapped glass sphere from the optical bottle.

The ability to open or close the bottle at will by varying the polarization of the input beam gives a unique opportunity to use the opening in the bottle not only to easily trap micro-object but also to unload the trap. Such functionality is demonstrated in Fig. 6.5. The image sequence represents various stages of closing the hole in the upper wall of the bottle while subsequently opening it in its bottom wall. It is clearly seen that the initially trapped sphere drops out of the trap under the effect of gravity when the hole in the bottom wall appears.

\subsection{Conclusions}

In summary, we have used the phenomenon of conical refraction of light to create an optical bottle for photophoretic trapping and manipulation of airborne particles. By changing the input beam polarization from circular to linear the light wall can be opened up to let the particles under study enter the otherwise almost impenetrable trap. If necessary, the trapped particles can later be released by rotating the plane of the linearly polarized input beam and thus adjusting the angular position of the exit opening in the trap. We have demonstrated the ability to perform such manipulations in ambient air with relatively large and heavy absorbing glass shells, in the range of $20 \mu \mathrm{m}-50 \mu \mathrm{m}$. Finally, we envisage that it would be very promising to combine the here presented technique with the results shown in Sec. 2.4 in order to reconfigure the CR optical bottle along the axial direction too, which could be used to vary dynamically the position of the trapped particles. 
CHAPTER 7

Trapping Bose-einstein condensates with conical refraction

In this Chapter we present two a novel proposals to trap ultracold neutral atoms and Bose-Einstein condensates (BECs) with dipole potentials generated with CR.

On the one hand, we demonstrate optical trapping of ultra-cold atoms in a bluedetuned trap formed by the three-dimensional dark focus shown in Sec. 3.2. We present experiments on trapping a ${ }^{87} \mathrm{Rb} \mathrm{BEC}$ in this potential and derive the trapping frequencies and potential barriers under the harmonic approximation and the CR theory. On the other hand, we also present a novel approach for the optical manipulation of neutral atoms in the Poggendorff dark ring of CR. We demonstrate both theoretically and experimentally that the Poggendorff dark ring is confined in three dimensions by regions of higher intensity. We derive the positions of the confining intensity maxima and minima and discuss the application of the Poggendorff ring for trapping ultra-cold atoms using the repulsive dipole force of blue-detuned light. We give analytical expressions for the trapping frequencies and potential depths along both the radial and the axial directions. Finally, we present realistic numerical simulations of the dynamics of a ${ }^{87} \mathrm{Rb} \mathrm{BEC}$ trapped inside the Poggendorff ring which are in good agreement with corresponding experimental results.

The organization of the Chapter is the following. In Sec. 7.1 we give an insight into atom trapping with red- and blue-detuned potentials based on the dipolar force. Sec. 7.2 and Sec. 7.4 are devoted, respectively, to discuss theoretically and demonstrate experimentally optical trapping of ${ }^{87} \mathrm{Rb}$ BECs in a blue-detuned 3D trap and in a bluedetuned light ring provided by $\mathrm{CR}$.

The research contained in this Chapter has been done in collaboration with Juan Polo, and Verònica Ahufinger from the Universitat Autònoma de Barcelona; and Johannes Küber, Felix Schmaltz and Gerhard Birkl from the Technische Universität Darm- 
stadt (TUD); and has been published in Refs. [178, 179]. The reported experiments were carried out by the author at the laboratory of Prof. Birkl at the TUD during a 3 months stay that was financed by the Deutscher Akademischer Austausch Dienst (grant number 91526836).

\subsection{Introduction}

The field of atomtronics is a key point to enable integrated matter-based quantum technologies such as quantum computation, quantum simulation and quantum metrology [180, 181]. Light-assisted manipulation of matter by means of the field intensity gradient throughout the dipolar force is one of the main techniques used in atomtronics. The intensity gradient of a light field can be used to efficiently trap ultra-cold atoms and BECs, using either red- or blue-detuned light. In the former technique, atoms are trapped in the region of intensity maxima taking profit of light whose wavelength is smaller (red-detuned case) or larger (bue-detuned case) than the atomic two-level transition frequency. Red-detuned (bright) optical traps are the most widely used optical traps due to their simplicity, since only a tightly enough focused beam producing a strong intensity gradient is needed. A drawback of trapped atoms in attractive dipole potentials is that they suffer from different energy shifts depending on the state and intensity of the trap. The fidelity of high precision measurements based on dipole traps also suffers from coherence loss caused by inhomogeneous differential light shifts [182]. In contrast, blue-detuned (dark) optical traps, allowing for a confinement of atoms in an intensity local minimum, have significantly decreased scattering rate and decoherence. Therefore, they are ideal candidates for highly sensitive experiments.

The aim of this chapter is to demonstrate that both a 3D dark potential and a dark ORP generated with CR can be used to efficiently trap BECs. Starting from the fundamental CR equations, we will obtain the trapping potentials and potential depths under the harmonic approximation. Then we will demonstrate experimental trapping of ${ }^{87} \mathrm{Rb}$ BECs in these potentials.

\subsection{Trapping BECs in a $3 \mathrm{D}$ dark focus}

Blue-detuned optical potentials are used in the manipulation of Rydberg states [183, atomic clocks [184], quantum information processing [185] or Bose-Einstein condensation in uniform potentials [186]. In the best ideal situation for blue-detuned optical traps, the local minimum where atoms are trapped has null intensity. If additionally this null intensity region is confined in three dimensions by regions of higher intensity, the light beam will form an optical bottle [95]. Different methods have been proposed to generate optical bottle beams, such as the interference of Laguerre-Gauss beams [99, surrounding a region of space in three dimensions with several beams [187, crossing two 
or more vortex beams [188] or by using optical C-cut uniaxial crystals [100]. However, most of these methods have associated different limitations such as the extreme precise control on the optical elements needed to generate and align the complex beams used or the fact that the intensity minimum is not exactly equal to zero [101. In what follows, we exploit the 3D dark focus presented in Sec. 3.2 as a blue-detuned potential for atom trapping experiments. Firstly, we will derive the trapping frequencies and potentials depths under harmonic approximation, by using the CR theory presented in Sec. 2.2 . Then we will report the experimental implementation of the CR 3D dark focus for the trapping of a ${ }^{87} \mathrm{Rb}$ BEC.

\subsubsection{Theoretical formulation for the 3D dark focus to atom trapping}
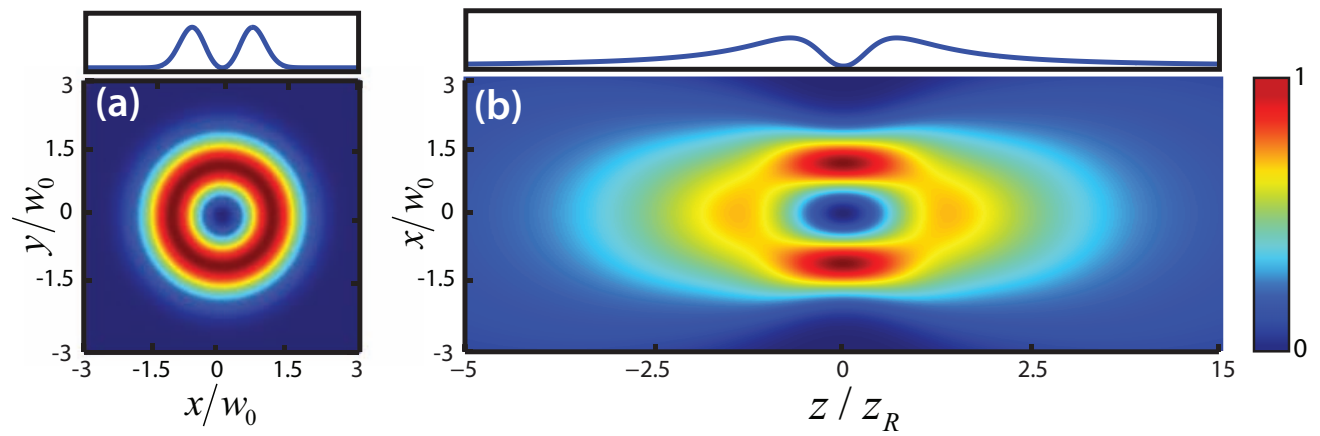

Figure 7.1: (a) 2D density plot of the transverse pattern of $\mathrm{CR}$ at the focal plane for $\rho_{0}=0.924$. (b) Corresponding $2 \mathrm{D}$ density plot in the $z-x$ plane showing that for this value of $\rho_{0}$ the CR beam forms a 3D dark focus. Top insets plot the radial and axial transverse cross-sections at $z=0$ and $x=0$, respectively.

Previously, it has been shown that for $\rho_{0}=R_{0} / w_{0}=0.924$ the CR beam forms a 3D dark focus, i.e., a null intensity point surrounded by regions of higher intensity in 3D space, as shown in Fig. 7.1. Now, we will study the behavior of the CR beam at and around the origin, i.e., for $\mathbf{r}=(\rho \approx 0, Z \approx 0)$, and we will derive the trapping frequencies and potential depths using the harmonic approximation. The dipolar potential trapping that it will be considered is [189]:

$$
\begin{aligned}
U(\mathbf{r}) & =-I(\mathbf{r}) \tilde{U}_{0}, \\
\tilde{U}_{0} & =\frac{\pi c^{2}}{2}\left[\frac{\Gamma_{D_{2}}}{\omega_{D_{2}}^{3}}\left(\frac{2}{\omega_{D_{2}}-\omega_{L}}\right)+\frac{\Gamma_{D_{1}}}{\omega_{D_{1}}^{3}}\left(\frac{1}{\omega_{D_{1}}-\omega_{L}}\right)\right] .
\end{aligned}
$$

Note that in $\tilde{U}_{0}$ we have applied the rotating-wave approximation and we consider the case of Alkali atoms. $c$ is light's velocity in vacuum, $\Gamma_{D_{i}}$ and $\omega_{D_{i}}(i=1,2)$ are, respectively, the natural line width and frequency of the $D_{i}$ line of the atomic specie used, and $\omega_{L}$ is the frequency of the input beam. In our case, $I(\mathbf{r})$, is given by Eqs. (2.12)(2.14). 


\section{Radial direction}

The Taylor expansion of the Bessel function of order $\alpha, J_{\alpha}(x)$, around $x=0$ can be written as follows:

$$
J_{\alpha}(x)=\sum_{k=0}^{\infty} \frac{(-1)^{k}}{k ! \Gamma(k+\alpha+1)}\left(\frac{x}{2}\right)^{2 k+\alpha},
$$

where $\Gamma(t)=\int_{0}^{\infty} x^{t-1} e^{-x} d x$ is the well known gamma function. Under this expansion and for an input beam of transverse fundamental Gaussian profile, Eqs. (2.12) and (2.13) become:

$$
\begin{aligned}
& B_{0}(\rho, Z)=E_{0} \int_{0}^{\infty} \eta e^{\frac{-\eta^{2}(1+i Z)}{4}} \cos \left(\eta \rho_{0}\right) \sum_{k=0}^{\infty} \frac{(-1)^{k}}{k ! \Gamma(k+1)}\left(\frac{\eta \rho}{2}\right)^{2 k} d \eta \\
& B_{1}(\rho, Z)=E_{0} \int_{0}^{\infty} \eta e^{\frac{-\eta^{2}(1+i Z)}{4}} \sin \left(\eta \rho_{0}\right) \sum_{k=0}^{\infty} \frac{(-1)^{k}}{k ! \Gamma(k+2)}\left(\frac{\eta \rho}{2}\right)^{2 k+1} d \eta
\end{aligned}
$$

where we have considered a Gaussian input beam with normalized transverse profile of the electric field amplitude $E(\rho)=E_{0} \exp \left(-\rho^{2}\right)$ and corresponding Fourier transform $a(\eta)=E_{0} \exp \left(-\eta^{2} / 4\right)$, being $E_{0}=\sqrt{\frac{P}{2 \pi w_{0}^{2}}}, P$ the power of the input beam and $w_{0}$ its waist radius. Eqs. (7.4) and (7.5) can be analytically solved, obtaining the following expressions:

$$
\begin{aligned}
& B_{0}(\rho, Z)=2 E_{0} \sum_{k=0}^{\infty} \frac{(-1)^{k} \rho^{2 k}}{k !(1+i Z)^{1+m}{ }_{1} F_{1}}\left(k+1 ; \frac{1}{2} ; \frac{-\rho_{0}^{2}}{1+i Z}\right) \\
& B_{1}(\rho, Z)=4 \rho_{0} E_{0} \sum_{k=0}^{\infty} \frac{(-1)^{k} \rho^{2 k+1}}{k !(1+i Z)^{m+1} 1} F_{1}\left(k+2 ; \frac{3}{2} ; \frac{-\rho_{0}^{2}}{1+i Z}\right) .
\end{aligned}
$$

where ${ }_{1} F_{1}(a ; b ; z)$ is the Kummer confluent hyper-geometric function. This formulation can be used for any value of $\rho_{0}$ as long as the point of intensity minimum remains at $\rho=0$. Note that the minimum intensity point $\rho_{\text {min }}$ will depend on the value of $\rho_{0}$. For the $3 \mathrm{D}$ dark focus $\left(\rho_{0}=0.924\right), \rho_{\min }=0$. We have found that close to $\rho=0$, the term $k=0$ approximates well to the original CR beam without approximation. In contrast, to describe appropriately the position of the radial maximum, up to the term $k=4$ must be considered. For the harmonic approximation, only up to second order terms are required. Since the intensity of the CR beam for a circularly polarized input beam is given by Eq. (2.14), it is enough to keep the $k=0$ terms of the series in Eqs. (7.7) and (7.8). In this case, the intensity of the $\mathrm{CR}$ beam reads as follows

$$
I(\rho \approx 0, Z)=\frac{2 P}{\pi w_{0}^{2}}\left(\left|\frac{{ }_{1} F_{1}\left(1 ; \frac{1}{2} ; \frac{-\rho_{0}^{2}}{1+i Z}\right)}{1+i Z}\right|^{2}+4 \rho_{0}^{2}\left|\rho^{2} \frac{{ }_{1} F_{1}\left(2 ; \frac{3}{2} ; \frac{-\rho_{0}^{2}}{1+i Z}\right)}{(1+i Z)^{2}}\right|^{2}\right) .
$$

The first term in Eq. (17.9) is an offset of the potential that appears when one moves out axially from the focal plane, as shown at the top inset of Fig. 17.1(b). As a consequence, 
trapping atoms out of the focal plane can increase the scattering rate of photons. The second term (after the ' + ') is the one that must be taken into account to obtain the trapping frequency of the potential. By using the harmonic approximation, the trapping frequency along the radial direction at any $Z, \omega_{r}(Z)$, is:

$$
\omega_{r}(Z)=\sqrt{\frac{16 \rho_{0}^{2} \tilde{U}_{0} P}{\pi m w_{0}^{4}}}\left|\frac{{ }_{1} F_{1}\left(2 ; \frac{3}{2} ; \frac{-\rho_{0}^{2}}{1+i Z}\right)}{(1+i Z)^{2}}\right|,
$$

where $m$ is the atomic mass. Note that, in the axial direction this approximation is only valid in the region where the optical bottle is formed, i.e,, for $Z \in[-1.388,1.388]$. Additionally, note that in Eq. (7.13), we have undone the normalization of the radial coordinate, i.e., we have replaced $\rho$ by $r / w_{0}$.

As commented above, the potential barrier along the radial direction, i.e., at the point $\mathbf{r}=(\rho=1.1, Z=0)$, is not well described by the harmonic approximation and it must be evaluated by taking terms $k>4$ in Eq. (7.9). Its value is:

$$
U(\rho=1.1, Z=0)=0.80 \times \tilde{U}_{0} \frac{P}{2 \pi w_{0}^{2}},
$$

\section{Axial direction}

For completeness, we will study also the trapping confinement along the axial direction. In this case, a compact expression for any value of $\rho_{0}$ cannot be obtained since the minimum radial intensity point depends on it. For our case of interest, the point of minimum intensity is at $\rho=0$. Here, the approximation from Eq. (7.3) used before is not needed since $J_{1}(0)=0$ and $J_{0}(0)=1$ and, as a consequence $B_{1}(\rho=0, Z)=0$. Therefore, the light intensity is solely described by $B_{0}$ as follows

$$
\begin{aligned}
I(0, Z)=\left|B_{0}(0, Z)\right|^{2} & =\frac{P}{2 \pi w_{0}^{2}}\left|\int_{0}^{\infty} \eta e^{-\frac{\eta^{2}(1+i Z)}{4}} \cos \left(\eta \rho_{0}\right) d \eta\right|^{2}= \\
& =\frac{2 P}{\pi w_{0}^{2}|1+i Z|}\left|1+\frac{2 \rho_{0} D\left(\frac{\rho_{0}}{\sqrt{1+i Z}}\right)}{\sqrt{(1+i Z)}}\right|^{2},
\end{aligned}
$$

where $D(x)$ is the Dawson function. The second order of the Taylor expansion of this analytical solution leads to the following expression for the trapping frequency $\left(\omega_{z}\right)$ along the axial direction:

$$
\omega_{z}=\sqrt{\frac{\tilde{U}_{0} P}{\pi m w_{0}^{2} z_{R}^{2}}} .
$$

The potential barriers along the axial direction, i.e., at $\mathbf{r}=(\rho=0, Z= \pm 1.388)$ can be obtained directly from Eq. (7.13). Their values are:

$$
U(\rho=0, Z= \pm 1.388)=0.32 \times \tilde{U}_{0} \frac{P}{2 \pi w_{0}^{2}},
$$




\subsection{Experimental trapping of a ${ }^{87} \mathrm{Rb} \mathrm{BEC}$ in the 3D dark focus}

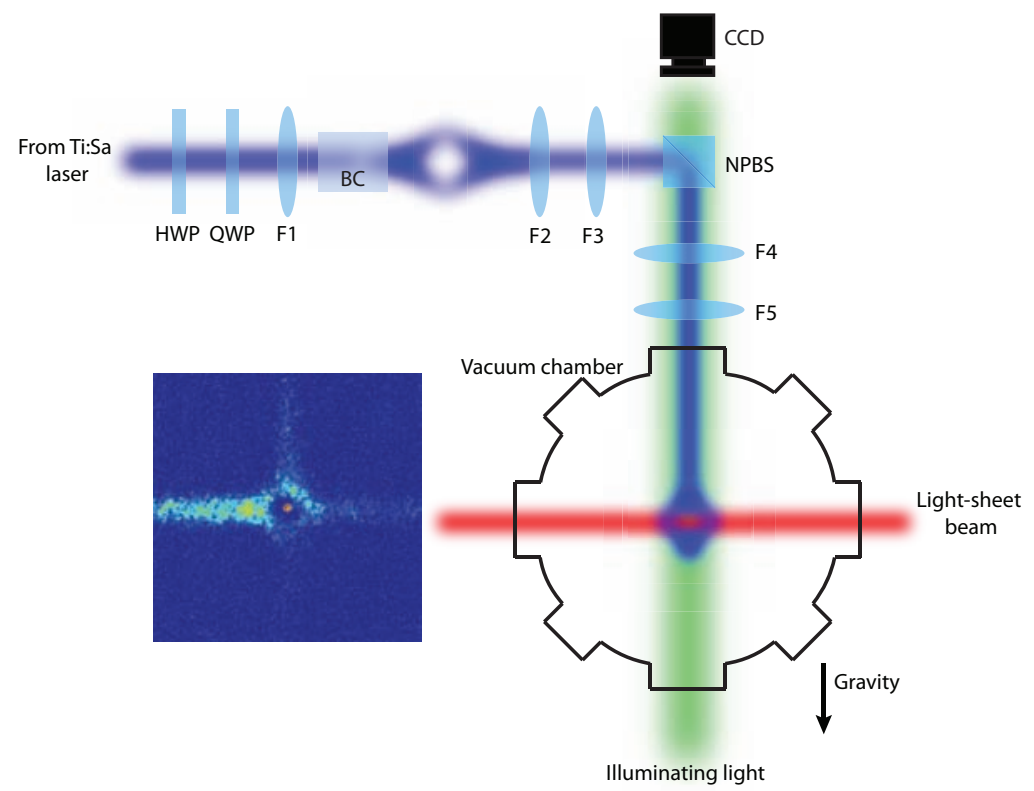

Figure 7.2: Experimental setup for the creation of a 3D dark focus potential based on CR. The CR ring potential $(\lambda=792.55 \mathrm{~nm})$ is oriented horizontally. Vertical confinement is achieved by a horizontally oriented red-detuned light sheet $(\lambda=783.55 \mathrm{~nm})$. The re-imaging system de-magnifies the $\mathrm{CR}$ beam by a factor of $0.75 . \mathrm{F}_{1}=150 \mathrm{~mm}$, $\mathrm{F}_{2}=\mathrm{F}_{3}=\mathrm{F}_{4}=400 \mathrm{~mm}, \mathrm{~F}_{5}=300 \mathrm{~mm}, \mathrm{HWP}=$ half- waveplate, QWP $=$ quarterwaveplate, NPBS = non-polarizing beam splitter. The inset is a density plot of an experimentally trapped ${ }^{87} \mathrm{Rb}$ BEC (image size $2 \mathrm{~mm} \times 2 \mathrm{~mm}$ ) with our set-up.

The experimental set-up is presented in Fig. 7.2, A $90^{\circ}$ mutually intersected pair of beams (not shown in the set-up) forming a crossed optical dipole trap is used to create a BEC of $25000{ }^{87} \mathrm{Rb}$ atoms in the center of the vacuum chamber. The crossing plane of the two beams is in the direction perpendicular to gravity. The light of the CR potential is obtained from a tunable Ti:Sapphire laser at $\lambda_{\mathrm{CR}}=792.55 \mathrm{~nm}$, providing a power of $P_{\mathrm{CR}}=24 \mathrm{~mW}$ inside the vacuum chamber. To generate the $3 \mathrm{D}$ dark focus potential, we align the focused input beam with waist radius of $42.7 \mu \mathrm{m}$ and Rayleigh length $z_{R}=5.42 \mathrm{~mm}$ along one of the optic axis of $\mathrm{KGd}\left(\mathrm{WO}_{4}\right)_{2}$ biaxial crystal by using a lens $F_{1}$ of $150 \mathrm{~mm}$ focal length. A half-waveplate (HWP) and a quarter-waveplate (QWP) are used to ensure the state of polarization of the input beam to be circular. A pinhole before the focusing lens $F_{1}$ is arranged in order to increase the spatial coherence of the input beam and ensure its Gaussian transverse profile. The $\mathrm{KGd}\left(\mathrm{WO}_{4}\right)_{2}$ crystal has a length of $l=2.2 \mathrm{~mm}$, a conicity of $\alpha(\lambda=792.55 \mathrm{~nm})=17.6 \mathrm{mrad}$ and, therefore, $R_{0}=37.4 \mu \mathrm{m}$. This value, together with the measured $w_{0}$, gives $\rho_{0}=0.88$, which is 
close to the theoretical value $\rho_{0}=0.924$ for the $3 \mathrm{D}$ dark focus. The transformed CR pattern appears at the focal plane of the system. Lenses $F_{2}, F_{3}, F_{4}$ and $F_{5}$ are used in telescope configuration to re-image the focal plane into the vacuum chamber on top of the crossed dipole trap. This re-imaging system de-magnifies the CR potential by a factor of 0.75 , so that the final radius of the $\mathrm{CR}$ ring and of the focused beam are $R_{0}^{\prime}=28.1 \mu \mathrm{m}$ and $w_{0}^{\prime}=32.0 \mu \mathrm{m}$, respectively.

The radial direction of the $3 \mathrm{D}$ dark focus is in a plane perpendicular to gravity. Since the confinement provided by the $3 \mathrm{D}$ dark focus depends on $z_{R}$, a large quantity of power would be required to trap atoms against gravity along the axial direction. For this reason, we use an additional red-detuned sheet of light generated by means of a cylindrical lens that focuses a Gaussian beam to hold atoms against gravity. For the light-sheet, we use light obtained from a diode laser at $\lambda_{\mathrm{LS}}=783.55 \mathrm{~nm}$, with a power of $P_{\mathrm{LS}}=137 \mathrm{~mW}$. The waist radius of the focused beam in the axial direction of the system is $w_{Z}=26.2 \mu \mathrm{m}$. All these parameters yield a measured trapping frequency of $\omega_{Z}=(169 \pm 2) \mathrm{Hz}$. This measurement was done by loading a BEC into the light sheet potential and switching it off for $300 \mu s$. Then, the light sheet was switched on again and we could observe the velocity of oscillation of the atoms in the potential induced by gravity. As a summary, our 3D optical trapping potential is formed by the dark focus with blue-detuned light, allowing for confinement in the radial direction, and for the light sheet with red-detuned light, allowing for confinement in the axial direction. The loading of the BEC into this potential is performed adiabatically, i.e., the crossed dipole trap is switched off slowly while the CR potential and the light sheet are switched on. The total duration time of the process is $40 \mathrm{~ms}$. Inset in Fig. 7.2 shows experimental atomic density images of a ${ }^{87} \mathrm{Rb} \mathrm{BEC}$ in this trapping configuration.

To measure the trapping frequency of the $3 \mathrm{D}$ dark focus potential we used the optical lattice to give a momentum of $2 \hbar k$ to the trapped atoms and measure their velocity for different oscillation times. We found a trapping frequency of $\omega_{R}=2 \pi \times(283 \pm 16) \mathrm{Hz}$. The experimental error is due to the azimuthal asymmetry of the potential heights.

To measure the potential heights of the blue-detuned dark focus trap, we used an optical lattice that accelerated the trapped atoms in a direction parallel to the lightsheet beam. In our experimental arrangement, both arms of the optical lattice could be modified independently both in intensity and frequency, resulting this in a moving optical lattice. More details about the optical lattice used can be found in Ref. [190]. The dark focus point of the CR potential was positioned on top of the crossing point of cross-dipole trap in order to accelerate the atoms against the light walls of the dark focus potential. Atoms were accelerated with a momentum of $2 \hbar k$ and we took measurements on the atom number that left the potential as a function of its power. The potential height is found when only half of the atoms can leave the potential. The measured potential height of the 3D trapping potential in the radial direction was $U_{r}=25 E_{R}$, where $E_{R}$ is the recoil energy $\left(E_{R} / \hbar=2 \pi \times 3.77 \mathrm{kHz}\right.$ in our experiments).

For the experimental parameters used in our experiment, the corresponding calcu- 
lated trapping frequency is $\omega_{r}\left(\rho_{0}=0.88, Z=0\right)=2 \pi \times(284) \mathrm{Hz}$, which is in complete agreement with the measured trapping frequency. With respect to the calculated potential barrier, we have obtained a theoretical value of $U(\rho=1.1, Z=0)=31 E_{R}$, which slightly differs with the corresponding measurement. This can be associated to the asymmetry of the maximum along the azimuthal direction in the experimental potential.

\subsection{Blue-detuned optical ring traps for BECs based on conical refraction}

Optical ring potentials (ORPs) with axial symmetry are considered as basic building blocks and the simplest nontrivial closed-loop circuits in atomtronics [180, 191, 194] and atom interferometry [195]. Atoms can be trapped by means of the optical dipolar force in high or low intensity regions with red-detuned [196, 197] or blue-detuned [198] light, in what follows called bright and dark potentials, respectively. On the one hand, bright ORPs have been proposed and demonstrated with high-azimuthal-order Laguerre-Gaussian (LG) beams [199] and also with annular microlenses [200, 201]. Azimuthal lattices within ORPs have been demonstrated with time orbiting of light beams [202,203] and by interference of LG beams of different azimuthal orders [204]. A one-dimensional stack of ORPs in a line has been proposed in an optical cavity [205] and demonstrated with axicon beams [206]. Experimental storage and propagation of ultra-cold atoms and BECs in bright ORPs have been reported recently [207, 208. Dark ORPs on the other hand are optical fields with an annular region of minimum intensity [170, such as closed-loop optical singularities [91,94, for which the region of minimum intensity is exactly zero. For ultra-cold atoms, dark ORPs have the advantage of substantially reducing atom heating and decoherence rates [198] because of the low rate of spontaneous photon scattering as well as producing intrinsically flat potential minima. Blue-detuned ORPs have been experimentally reported by means of LG beams generated with spatial light modulators (SLMs) 209] and by amplitude masks [210 212 . These two techniques might experience the following limitations: (i) a significant fraction of the input power is lost and, therefore, it does not contribute to create the optical trap, (ii) the smoothness and, therefore, the quality of the trapping potential is limited by the size and number of pixels for the SLMs and the resolution of the printing system for the amplitude masks, and (iii) an accurate control on the position and alignment of the optical elements being used is required. As a consequence, these two techniques yield typically not null intensity minima. Producing ORPs with zero-intensity annular regions both along the radial and axial directions is a challenging task. In this case, the dark potential forms a toroidal dark focus, i.e., a region of minimum intensity confined by higher intensities (light walls) both in the axial and radial directions. A toroidal dark focus has only been demonstrated using a superposition of two LG beams [170].

In this section, we present a new method to generate a dark ORP by means of the 
Poggendorff dark ring (PDR) of CR. We theoretically investigate the three-dimensional (3D) field distribution around the CR PDR and show both theoretically and experimentally that it is a toroidal dark focus. We also discuss the use of the PDR as a blue-detuned ORP for ultra-cold atoms and demonstrate this for a ${ }^{87} \mathrm{Rb}$ BEC.

\subsubsection{Asymptotic solution close to the Poggendorff dark ring}

The asymptotic solution for the Poggendorff dark ring, i.e., for $\rho_{0}=\frac{R_{0}}{w_{0}} \gg 1$, is obtained by using the asymptotic expansion of Bessel functions: $\cos \left(\eta \rho_{0}\right) J_{0}(\eta \rho) \approx$ $\sin \left(\eta \rho_{0}\right) J_{1}(\eta \rho) \approx \cos (\eta \xi-\pi / 4) / \sqrt{2 \pi \eta \rho_{0}}$. Here we have centered the normalized radial component in cylindrical coordinates at $\rho_{0}$ by using $\xi \equiv \rho-\rho_{0}=r / w_{0}-R_{0} / w_{0}$. In this case $B_{C} \approx B_{S}$ and the electric field can be written as [27, 29]:

$$
\mathbf{E}(\xi, Z, \varphi)=f(\xi, Z) E_{0}\left(\mathbf{e}_{\mathrm{CR}} \cdot \mathbf{e}_{0}\right) \mathbf{e}_{\mathrm{CR}},
$$

where

$$
f(\xi, Z)=\sqrt{\frac{1}{8 \pi^{3} \rho_{0}}} \quad \int_{0}^{\infty} d \eta \sqrt{\eta} a(\eta) e^{-i \frac{Z}{4} \eta^{2}} \cos \left(\eta \xi-\frac{\pi}{4}\right)
$$

and

$$
\mathbf{e}_{\mathrm{CR}}=\left(\begin{array}{c}
\cos \frac{\varphi+\varphi_{0}}{2} \\
\sin \frac{\varphi+\varphi_{0}}{2}
\end{array}\right) .
$$

Therefore, the asymptotic intensity distributions $I_{\mathrm{CP}}^{\mathrm{a}}$ and $I_{\mathrm{LP}}^{\mathrm{a}}$ for $\mathrm{CP}$ and LP input beams are, respectively,

$$
\begin{aligned}
I_{\mathrm{CP}}^{\mathrm{a}}(\xi, Z) & =|f(\xi, Z)|^{2}, \\
I_{L P}^{\mathrm{a}}(\xi, Z, \varphi) & =I_{\mathrm{CP}}^{\mathrm{a}} \cos ^{2}\left(\Phi-\frac{\varphi+\varphi_{0}}{2}\right) .
\end{aligned}
$$

In the following we will analyze the case of a CP input beam, for which the CR output intensity is azimuthally symmetric and its spatial distribution is described by Eq. (7.18). For the Gaussian input beam considered in the previous section, Eq. (7.16) can be analytically evaluated through the Kummer confluent hyper-geometric function ${ }_{1} F_{1}(a ; b ; z)[32$ :

$$
\begin{aligned}
f(\xi, Z) & =\frac{\sqrt{P}}{(1+i Z)^{3 / 4} \sqrt{2 \pi^{2} w_{0}^{2} \rho_{0}}}\left[\Gamma\left(\frac{3}{4}\right){ }_{1} F_{1}\left(\frac{3}{4} ; \frac{1}{2} ;-\frac{\xi^{2}}{1+i Z}\right)\right. \\
& \left.+2 \frac{\xi}{\sqrt{1+i Z}} \Gamma\left(\frac{5}{4}\right){ }_{1} F_{1}\left(\frac{5}{4} ; \frac{3}{2} ;-\frac{\xi^{2}}{1+i Z}\right)\right],
\end{aligned}
$$

where $w_{Z}=1+i Z$. 

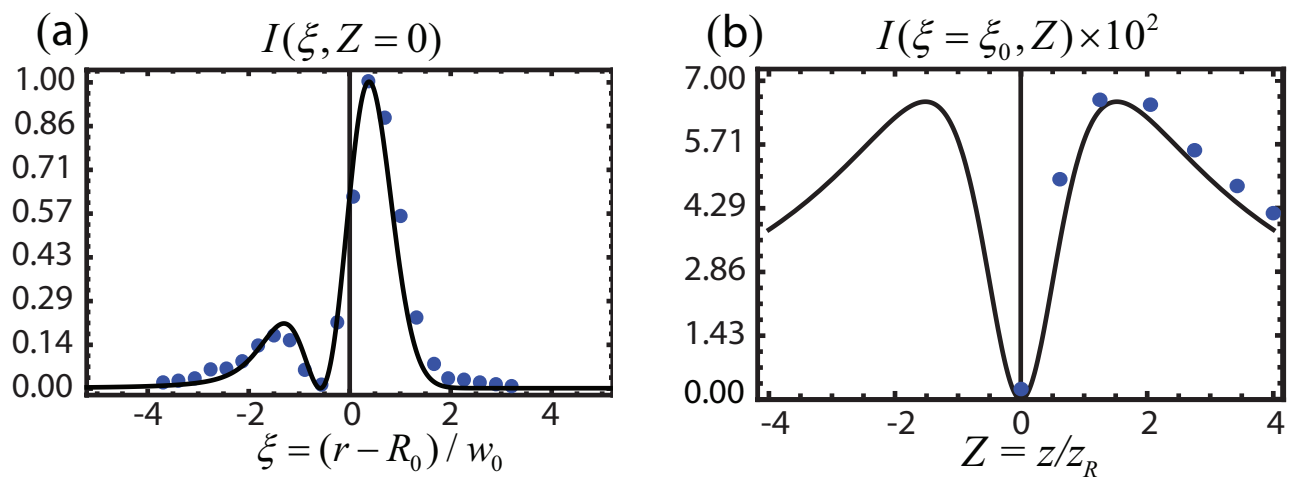

Figure 7.3: Normalized CR intensity for a CP Gaussian input beam as given by Eq. (7.20) along the radial direction (a) at the focal plane and (b) along the axial direction at the radial position of the $\operatorname{PDR}\left(\xi=\xi_{0}\right)$. Blue solid circles represent experimental data with an experimental uncertainty of $5 \%$ along both axis.

The solid line in Fig. 7.3(a) shows the square modulus of Eq. (7.20) at the focal plane $(Z=0) . f\left(\xi_{0}, 0\right)=0$, gives the radial position of the Poggendorff dark ring at the focal plane, being $\xi_{0}=-0.541$. In other words, the radius of the PDR is smaller than the geometric approximation of the CR ring, $R_{0}$, by approximately half the waist of the input beam. Note that $\xi=\rho-\rho_{0}$, with $\rho_{0} \equiv R_{0} / w_{0}$. In the radial direction the PDR is confined by two maxima at $\xi_{+}=0.390$ and $\xi_{-}=-1.235$, respectively (see Table (7.1). Along the $\mathrm{Z}$ direction, the lowest intensity barrier is observed also at the radial position of the PDR, i.e., at $\xi=\xi_{0}$ as shown in Fig. 7.3(b). At this radial point the positions of the intensity maxima along $Z$ obtained from Eq. (7.18) and (7.20) are $Z_{ \pm}= \pm 1.519$. Therefore, the PDR is confined by walls of light in all directions and forms a toroidal dark-focus. Table 7.1 presents the positions of the PDR and of the maxima in the radial $\left(\xi_{ \pm}\right)$and axial $\left(Z_{ \pm}\right)$directions. As a visualization of the toroidal dark

Table 7.1: Positions of the Poggendorff dark ring and of the maxima in the radial $(\xi \pm)$ and axial $(Z \pm)$ directions.

\begin{tabular}{rcc}
\hline Point name & $\xi\left(w_{0}\right)$ & $Z\left(z_{R}\right)$ \\
\hline Dark Ring: $\xi_{0}$ & -0.541 & 0 \\
Bright Rings: $\xi_{+}$ & 0.390 & 0 \\
$\xi_{-}$ & -1.235 & 0 \\
Maxima along Z: $Z_{ \pm}$ & -0.541 & \pm 1.519
\end{tabular}

trap provided by the PDR of CR, Fig. 7.3(a) shows the three-dimensional distribution of light intensity of the asymptotic approximation of the BKB solution near the focal plane. Fig. 7.3(b) is a contour plot near the PDR, confirming that it is a region of low intensity surrounded in all directions by regions of higher intensity. Note that the PDR is an exact null intensity region only for input Gaussian beams under the asymptotic 
approximation, i.e., for $\rho_{0} \gg 1$, while non-zero intensity radial minimum points are found out of the paraxial approximation, as reported in Chapter 3. For input beams with different transverse profile the CR pattern may change [70,72].

(a)

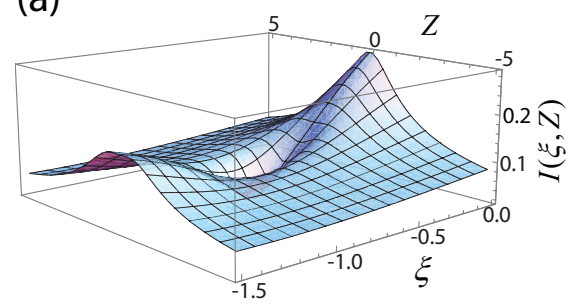

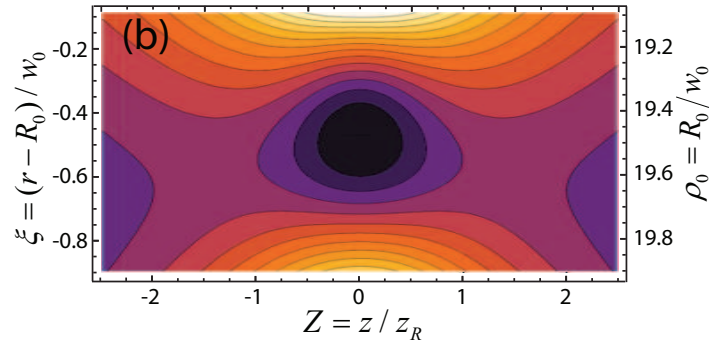

Figure 7.4: (a) Normalized light intensity in three dimensions near the PDR. (b) 2D contour density plot near the PDR of the normalized light intensity calculated from Eqs. (7.18) and (7.20) and for $\rho_{0}=R_{0} / w_{0}=20$. Color map: black $=$ null intensity, white $=$ high intensity.

We have experimentally checked that near the PDR the light intensity increases in all directions, see blue solid circles in Fig. 7.4(a) and Fig. 7.4(b). These experiments on the CR PDR were carried out using a CP focused input Gaussian beam $\left(w_{0}=40 \mu \mathrm{m}\right.$, $\left.z_{R}=7.9 \mathrm{~mm}\right)$ at $\lambda=640 \mathrm{~nm}$ and a $\mathrm{KGd}\left(\mathrm{WO}_{4}\right)_{2}$ biaxial crystal (cross-section $6 \times 4 \mathrm{~mm}^{2}$, $l=28 \mathrm{~mm}, \alpha=16.9 \mathrm{mrad}$ ) cut perpendicular to one of the optic axes (entrance surface parallelism better than 10 arc seconds) yielding a $\mathrm{CR}$ ring of $R_{0}=475 \mu \mathrm{m}\left(\rho_{0} \approx 12\right)$. The transverse light patterns at and around the focal plane were recorded with a CCD camera.

\subsubsection{Harmonic potential approximation}

We consider the same potential as described by Eq. (17.1) and Eq. (7.2) where $I(\mathbf{r})$ is given by Eq. (17.18) and Eq. (7.20). By using the harmonic approximation, we have obtained the following expressions for the corresponding radial $\left(\omega_{r}\right)$ and axial $\left(\omega_{z}\right)$ trapping frequencies of the $\operatorname{PDR}\left(\xi=\xi_{0}, Z=0\right)$

$$
\omega_{r, z}=\sqrt{\frac{A_{r, z} \tilde{U}_{0} P}{\pi^{2} m w_{0}^{4} \rho_{0}}},
$$

with the numerical constants $A_{r}(Z=0)=4.63$ and $A_{z}=0.34$. Eq. (7.21) is obtained by expanding Eq. (7.20) in Taylor series, introducing it into Eq. (17.18) and considering the $\xi^{2}$ coefficient.

Note that from Eqs. (7.18), (7.20), and (7.21) for a given CR set-up, i.e., for a fixed $R_{0}$ and $w_{0}$, the trapping frequencies and the maxima of the potential barriers can be tuned by modifying the power $P$ and the frequency $\omega_{L}$ of the input beam. We have 
obtained that at the focal plane the maxima of the potential barriers are described by

$$
U\left(\xi_{ \pm}, 0\right)=C_{ \pm} \tilde{U}_{0} \frac{P}{4 \pi^{2} w_{0}^{2} \rho_{0}},
$$

where $C_{+}=2.54$ (outer bright ring) and $C_{-}=0.541$ (inner bright ring).

There can be other experimental situations however, where it is required to work outside the focal plane, for instance in experiments where a more symmetric potential is needed, such as the one shown with a solid line in Fig. 17.5(a), where the radial intensity distribution close to the PDR is shown for the focal plane $(Z=0)$ and the plane $Z=4$. In these cases, Eq. (7.21) can be utilized to calculate the trapping frequency of the potential at any axial position $Z$ by just replacing $A_{r}(Z=0)$ by

$$
A_{r}(Z)=-0.051+\frac{8.817}{1.873+2.307 Z^{2}} .
$$

Figure 7.5(b) presents the dependence of $A_{r}(Z)$ on $Z$. Note that outside the focal plane an offset to the potential is occurring, since the minimum intensity point is no longer of null intensity as plotted for $Z=4$ as solid line in Fig. 7.5(a). We have found that this non-zero minimum intensity point can be taken into account by means of the optical potential along the axial direction,

$$
U\left(\xi_{0}, Z\right)=\tilde{U}_{0} \frac{P}{4 \pi^{2} w_{0}^{2} \rho_{0}} Z^{2} .
$$

The confining maxima along $Z$ are not well described by the harmonic approximation and must be evaluated using Eqs. (7.18) and (7.20). They read

$$
U\left(\xi_{0}, Z_{ \pm}\right)=0.17 \tilde{U}_{0} \frac{P}{4 \pi^{2} w_{0}^{2} \rho_{0}} .
$$
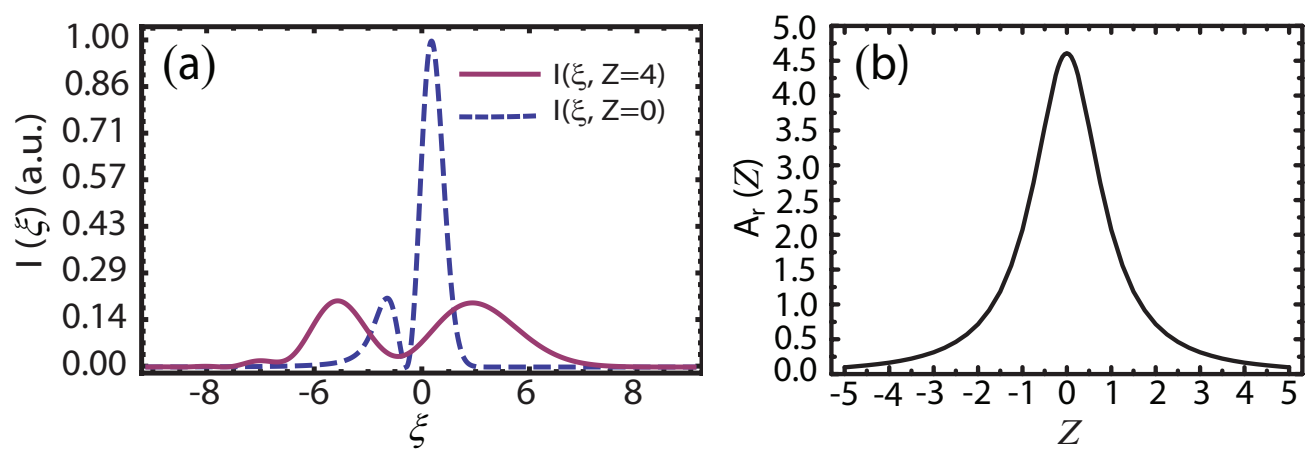

Figure 7.5: (a) Profile of the trapping potential at $Z=0$, i.e., at the focal plane (dashed curve), and at $Z=4$ (solid curve) where the inner and the outer bright rings of CR have equal maximum intensity. (b) Coefficient $A_{r}$ as a function of $Z$. The analytical expression for the $A_{r}(Z)$ is given by Eq. (7.23). 


\subsubsection{Numerical simulations of a BEC of ${ }^{87} \mathrm{Rb}$ atoms}

To demonstrate the applicability of the PDR for ultra-cold gases, now we discuss the two-dimensional (2D) evolution of a BEC of ${ }^{87} \mathrm{Rb}$ atoms confined in an annular geometry within the focal plane by using the PDR of CR and a strong additional confinement along the axial direction so that $\omega_{\text {axial }} \gg \omega_{r}$. Such confinement can be achieved by using an additional red-detuned sheet of light (e.g. generated by focusing a Gaussian beam with a cylindrical lens as shown in Fig. (7.2) to compensate for the weak axial confinement as well as, in case of a horizontal ring plane, the effect of gravity. We use the 2D GrossPitaevskii equation (GPE) in order to study the dynamics of the BEC along the ring potential:

$$
i \hbar \frac{\partial}{\partial t} \Psi(\mathbf{r}, t)=\left(-\frac{\hbar^{2}}{2 m} \nabla^{2}+V_{\text {ext }}(\mathbf{r})+g_{2 D}|\Psi(\mathbf{r}, t)|^{2}\right) \Psi(\mathbf{r}, t)
$$

where $V_{\text {ext }}(\mathbf{r})$ is the external potential, $g_{2 D}=2 \hbar a_{s} N \sqrt{2 \pi \frac{\hbar \omega_{z}}{m}}, a_{s}$ is the scattering length, $\omega_{z}$ is the frequency of the confining potential in the axial direction, $m$ is the mass of the ${ }^{87} \mathrm{Rb}$ atoms and $N$ is the number of atoms.

In our simulations, we consider trapping close to the $D_{2}$ and $D_{1}$ lines of ${ }^{87} \mathrm{Rb}$. These lines possess natural line widths of $\Gamma_{D_{2}}=2 \pi \times 6.07 \mathrm{MHz}$ and $\Gamma_{D_{1}}=2 \pi \times 5.75 \mathrm{MHz}$ and frequencies of $\omega_{D_{2}}=2 \pi \times 384.23 \mathrm{THz}$ and $\omega_{D_{1}}=2 \pi \times 377.11 \mathrm{THz}$, respectively. Thus, to calculate the trapping frequencies and the maxima of the potential barriers is straightforward by using Eqs. (7.2), (7.21), (7.22), and (7.25). Based on the experimental parameters of [190, for a biaxial crystal yielding a CR ring of $R_{0}=170 \mu \mathrm{m}$, an input beam waist $w_{0}=18 \mu \mathrm{m}$, a light frequency of $\omega_{L}=2 \pi \times 378.40 \mathrm{THz}$ and a laser power $P=27 \mathrm{~mW}$, at the focal plane, the maxima of the potential barriers and trapping frequencies are, respectively, $U\left(\xi_{-}, Z=0\right) / k_{B}=280 \mathrm{nK}, U\left(\xi_{+}, Z=0\right) / k_{B}=1314 \mathrm{nK}$ and $\omega_{r}=2 \pi \times 265 \mathrm{~Hz}$, where $k_{B}$ is the Boltzmann constant.

Figure 7.6(a) shows the numerical simulation for a ${ }^{87} \mathrm{Rb}$ BEC, with scattering length $a_{s}=5.45 \mathrm{~nm}$, of $N=12000$ atoms trapped in a blue-detuned harmonic annular potential $V_{r}=\frac{1}{2} m \omega_{r}^{2}\left(r-\left(R_{0}-0.541 w_{0}\right)\right)^{2}$ with radial frequency $\omega_{r}=2 \pi \times 265 \mathrm{~Hz}$ calculated using Eq. (7.21). Our numerical simulations are based in the following loading process: the BEC is created in a cross-dipole trap, see e.g. [213, and loaded into the red-detuned sheet of light. We consider that both the cross-dipole trap and the red-detuned sheet of light lie in a common plane orthogonal to the gravity field. The PDR potential, which also lies in the plane orthogonal to the gravity field, is placed tangent to one of the beams of the cross-dipole trap, see Fig. 7.6(c). The beam from the cross-dipole trap that is tangent to the PDR is switched off as the CR PDR potential is switched on, in an adiabatic process. Finally, the remaining beam from the cross-dipole trap is switched off and the BEC expands in the CR PDR potential. We plot the atomic density of the $\mathrm{BEC}$ after $30 \mathrm{~ms}$ of expansion in the annular potential. In order to reduce the transverse excitations, the loading of the $\mathrm{BEC}$ into the $\mathrm{CR}$ ring potential has been performed adiabatically (in our case during $20 \mathrm{~ms}$ ) as reported in [190]. Fig. 7.6(b) shows the 
corresponding experimental density distribution after $30 \mathrm{~ms}$ expansion of a ${ }^{87} \mathrm{Rb} \mathrm{BEC}$ trapped in the real CR PDR. The CR PDR was placed perpendicular to gravity and a sheet of light generated by focusing a Gaussian beam with a cylindrical lens was used to hold atoms against gravity. The corresponding measured trapping frequencies are $\omega_{r}^{\exp }=2 \pi \times(300 \pm 20) \mathrm{Hz}$ and $\omega_{z}^{\exp }=2 \pi \times(169 \pm 2) \mathrm{Hz}$
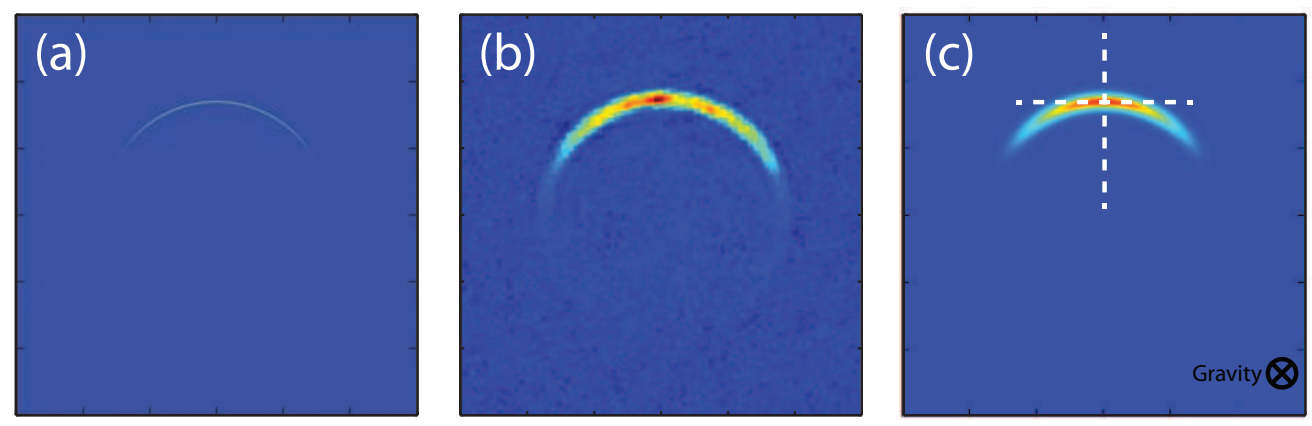

Figure 7.6: (a) Plot of the atomic density from the numerical simulation of a trapped ${ }^{87} \mathrm{Rb} \mathrm{BEC}$ after $30 \mathrm{~ms}$ of expansion in the ring $V_{r}=\frac{1}{2} m \omega_{r}^{2}\left(r-\left(R_{0}-0.541 w_{0}\right)\right)^{2}$, with the frequency $\omega_{r}=2 \pi \times 265 \mathrm{~Hz}$ being calculated using the harmonic approximation. Parameter values used for the simulation: $R_{0}=170 \mu \mathrm{m}, w_{0}=18 \mu \mathrm{m}, P=27 \mathrm{~mW}$, $w_{z}=2 \pi \times 500 \mathrm{~Hz}, a_{s}=5.45 \mathrm{~nm}$ and $N=12000$ atoms. (b) Experimental density distribution of a trapped ${ }^{87} \mathrm{Rb} \mathrm{BEC}$ in the $\mathrm{CR}$ ring potential using the same experimental parameters as for the numerical simulation, with the exception of the axial confinement, that was made using a red-detuned Gaussian beam focused with a cylindrical lens, providing a measured trapping frequency of $w_{z}^{\exp }=2 \pi \times(169 \pm 2) \mathrm{Hz}$. The measured radial trapping frequency provided by the CR PDR was $\omega_{r}^{\exp }=2 \pi \times(300 \pm 20) \mathrm{Hz}$. (c) Numerical simulation under the same conditions as in (a) but including the scattering induced by the position spreading during detection. Each figure is $600 \mu \mathrm{m} \times 600 \mu \mathrm{m}$. Color map: dark blue (red) corresponds to null (high) intensity. White dashed lines in (c) indicate the position of the cross-dipole trap with respect to the PDR, being both of them orthogonal to the gravity field. The waist radius of each beam from the cross-dipole trap is $25 \mu \mathrm{m}$.

The major discrepancy between experimental and numerical density plots is found in the radial width of the BEC. In the ideal case (Fig. 7.6(a)), the effects of broadening due to finite optical resolution and photon scattering of the detection light have not been considered to obtain the plot, which shows a BEC with a width of $3 \mu \mathrm{m}$. In contrast, the experimental image from Fig. 7.6(b), which shows a BEC with a width of $25 \mu \mathrm{m}$, was obtained by using red-detuned light $\left(\lambda_{\text {ill }}=780 \mathrm{~nm}, P_{\text {ill }}=0.25 \mathrm{~mW}\right)$ to illuminate the BEC during a time of $t_{\text {ill }}=200 \mu \mathrm{s}$. For this illuminating light we have calculated a scattering rate $\Gamma_{s c}=3.29 \times 10^{6} \mathrm{~s}^{-1}$ that, together with the recoil velocity of $v_{\text {rec }}=5.89 \mathrm{~mm} / \mathrm{s}$, increases the width of the atomic cloud in the radial direction by $21.89 \mu \mathrm{m}$ during the illumination time. Figure 7.6(c) shows the same numerical 
simulation as Fig. 7.6(a) where we have taken into account now the increase of the width produced by the detection process. Now, numerical simulation and experimental result agree well.

In order to further confirm the validity of the harmonic approximation, we also studied the ground state of the BEC trapped in the toroidal dark-focus (see Fig. (7.7)). The physical system considered has the following parameters: $R_{0}=170 \mu \mathrm{m}, w_{0}=$ $18 \mu \mathrm{m}, P=27 \mathrm{~mW}, w_{r}=2 \pi \times 265 \mathrm{~Hz}, a_{s}=5.45 \mathrm{~nm}$ and $N=12000$ atoms. The toroidal dark trap is placed orthogonal to gravity and, to provide confinement along the axial direction, we have considered a sheet of light analogous to the one discussed in [190] with a trapping frequency $w_{z}=2 \pi \times 500 \mathrm{~Hz}$. The plots represent a section of the wave-function probability in the radial direction at the peak value of the density. The red-solid line in Fig. 7.7(a) shows the ground state wave-function probability of the BEC trapped in the PDR potential (represented by the red-dashed line), while the black-solid line is the ground state of the BEC trapped in the harmonically approximated potential (represented by black-dashed line) equivalent to the PDR. To provide confinement in the azimuthal direction, an extra beam yielding a trapping frequency of $w_{\text {azi }}=2 \pi \times 265 \mathrm{~Hz}$ is included. We have found a $0.7 \%$ of relative difference between the energies of the two ground states. Figure [7.7(b) presents the BEC wave-function probability after $30 \mathrm{~ms}$ of expansion within the harmonically approximated ring potential (black-solid line) and within the real PDR potential (red-solid line). Black- and red-dashed lines represent the harmonic ring potential and the PDR potential, respectively. In this case, the relative difference between both wave-function probabilities is negligible. These results confirm the good agreement between the harmonic approximation derived above and the original PDR.
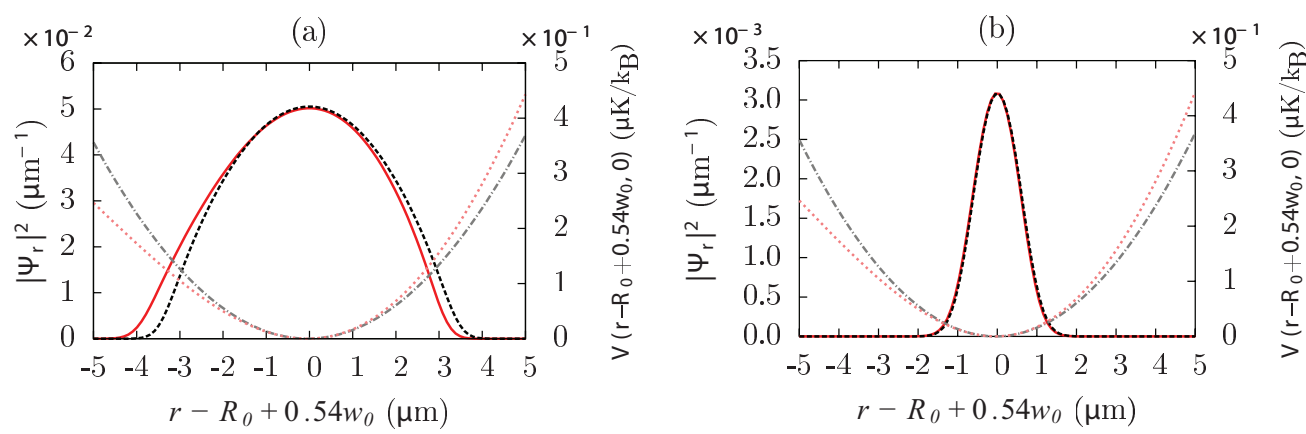

Figure 7.7: Radial sections of the atomic density of the BEC (a) before and (b) after $30 \mathrm{~ms}$ of azimuthal expansion of the BEC trapped in the harmonic potential $V_{r}=\frac{1}{2} m \omega_{r}^{2}\left(r-\left(R_{0}-0.541 w_{0}\right)\right)^{2}$ (black-dashed line) and in the Poggendroff dark ring of CR (red-solid line). Black-dashed and red-dotted lines are the corresponding trapping potentials. Parameter values: $R_{0}=170 \mu \mathrm{m}, w_{0}=18 \mu \mathrm{m}, P=27 \mathrm{~mW}, w_{r}=2 \pi \times 265 \mathrm{~Hz}$, $w_{z}=2 \pi \times 500 \mathrm{~Hz}, a_{s}=5.45 \mathrm{~nm}$ and $N=12000$ atoms. The ground state (a) is obtained by adding an extra confinement $\left(w_{\mathrm{azi}}=2 \pi \times 265 \mathrm{~Hz}\right)$ in the azimuthal direction in order to reproduce the loading of the $\mathrm{BEC}$ in the $\mathrm{CR}$ ring trap. 


\subsection{Conclusions}

In this Chapter we have proposed and demonstrated two blue-detuned novel optical potentials based on CR for atomtronics experiments. On the one hand, we have demonstrated the experimental implementation of a blue-detuned 3D trap obtained from a single beam and a biaxial crystal. We have derived simple expressions for the trapping frequencies and potential barriers in three dimensions as a function of typical experimental parameters. Both experimental measurements and predicted results are in complete agreement with each other. In our case, the $3 \mathrm{D}$ dark focus beam was arranged with the beam propagation direction parallel to gravity. Since the axial confinement offered by such optical potential is not enough to compensate gravity, an additional trapping potential was needed to confine in this direction was needed. However, the $3 \mathrm{D}$ dark focus can be used as a blue-detuned optical trap with a single beam by arranging the light potential in a plane orthogonal to gravity. One of the advantages of our technique is that CR provides the full conversion of the input power into the 3D dark focus and avoid diffraction losses, in contrast to other methods based on spatial light modulators (SLMs), for instance, which introduce losses due to diffraction in the generation of LG beams. Moreover, biaxial crystals can be transparent to an extremely wide spectral range 214] $\left(0.35 \mu \mathrm{m}-5.5 \mu \mathrm{m}\right.$ in $\mathrm{KGd}\left(\mathrm{WO}_{4}\right)_{2}$, for instance), at variance with SLMs which only work in a short spectral range usually of few hundreds of $\mathrm{nm}$. These features make the 3D dark focus beam very attractive for particle manipulation [142, 143] and atom [185, 200] trapping. We would like to note that if instead of a Gaussian input beam, an elliptical beam was used, the 3D dark focus would lead to a pair of elliptical beams divided by a thin dark region. This configuration could be used as a dark sheet potential. Also, a combination of the 3D dark focus with an array of micro lenses [201,215] would lead to the generation of a 2D array of 3D dark traps of significant interest in atom trapping for quantum computing experiments. Finally, the interference of the 3D dark focus with a plane wave along the beam propagation direction would generate a $1 \mathrm{D}$ stack of doughnut-like beams, ideal for quantum many-body experiments [206].

On the other hand, we have demonstrated that the Poggendorff dark ring of CR obtained for $\rho_{0} \gg 1$ is a good candidate for matter-wave experiments requiring annular geometries. We have found the positions of the bright and dark rings of CR and the position of the two points with maximum intensity along the beam propagation direction, both experimentally and analytically. Besides, we have shown that the radius of the $\mathrm{PDR}$ is smaller than the optical geometric approximation of the CR ring radius $R_{0}$, by approximately half the waist radius of the input beam $\left(-0.541 w_{0}\right.$ in Table 7.1). All previous related works $[27,29,31,32,74$ ] were performed considering that the radius of the PDR exactly coincided with $R_{0}$. The reported results show that the PDR is enclosed by higher intensity walls both in the radial as in the axial directions, i.e., it is a toroidal dark-focus in all three dimensions, at variance with other light beams possessing only radial confinement, such as Laguerre-Gaussian modes. We have applied the harmonic approximation around the PDR and we have derived the expression for the 
radial and axial trapping frequencies and the maxima of the potential barriers for bluedetuned light as a function of common experimental parameters such as beam power, beam waist, detuning and the parameters of the crystal. The reported results show the suitability of the PDR for trapping ultra-cold atoms with blue-detuned light, making this technique ideal for experiments where well-defined potentials and high intensity beams are required [170,198, 200]. Therefore, as a proof of the usefulness of the derived theory we have performed numerical simulations of the dynamics of a trapped ${ }^{87} \mathrm{Rb} \mathrm{BEC}$ with $N=12000$ atoms in the dark ring potential using the harmonic approximation and have compared the obtained results with the solution of the original CR light field. We have also compared the ground states in both cases and we have found $0.7 \%$ relative difference in energy between them. The numerical simulations agree well with the experimental results on the dynamics of a trapped ${ }^{87} \mathrm{Rb} \mathrm{BEC}$ in the PDR of $\mathrm{CR}$. In addition to the advantages commented above in terms of power efficiency and beam quality, the minimum (and practically null) intensity circle offered by the toroidal dark trap avoids photon scattering and presents no corrugation of the potential minimum at the focal plan, at variance with techniques based on LG beams [209] or amplitude masks [210212. A range of applications of this technique can be envisioned: for optimized beam geometries, i.e., small $w_{0}, R_{0}$, and $z_{R}$, the toroidal dark focus of the PDR generated by $\mathrm{CR}$ could be used to built an all-optical trap for BECs using a single beam. Under such conditions, this potential could be used as a basic element in atomic SQUID experiments [216, 217], as well as to study the dynamics of matter waves with periodic boundary conditions and the generation of persistent currents [208, 218]. For large $R_{0}$, the PDR can be used as a dark 2D ring potential by using a 1D light sheet, along the axial direction, as an additional confining potential. This configuration would allow to study wave-packet interference in a mesoscopic ring simulating a quasi-one-dimensional system. By modifying this 1D light sheet to a blue-detuned double layer also accessible via CR [54,66] again a fully blue-detuned dark trap geometry with added flexibility is generated. Additionally, a radial optical lattice could be generated by means of a cascade of biaxial crystals, generating $2^{N-1}$ dark rings for a cascade of $\mathrm{N}$ biaxial crystals [55, 83]. This could be also combined with the technique shown in 82 to generate an azimuthal optical lattice with controllable number of nodes and separation between them, applicable in quantum-many body systems experiments [219,220]. Also interesting is the possibility of using the PDR to coherently injecting, extracting, and velocity filtering of particles, ultra-cold atoms and BECs as reported in [142,144] by tuning the polarization of the input beam and opening/closing the ring potential. Finally, we would also like to note that by switching to red-detuned light, the inner and outer bright rings around the PDR generate an intrinsically concentric system of a double-ring potential which can be used for the generation of coherent double wave packets for the investigation of wave packet tunneling and coupled persistent currents of ultra cold atoms [221,222]. 

CHAPTER 8

Second harmonic generation of a conically refracted beam

Type I and type II second harmonic generation (SHG) of a beam transformed by the conical refraction phenomenon are presented within this Chapter. At variance with other works combining SHG and CR where both phenomena are generated in a single non-linear biaxial crystal, see Section 8.1, in our approach we firstly transform an input Gaussian beam with a linear biaxial crystal and then double its frequency with nonlinear KTP and LBO crystals. In this case, a very efficient process is found, since the optic axis of the linear biaxial crystal can be aligned with the phase-matching direction of the non-linear crystals used. Section 8.2 describes our experimental approach, in which we use LBO (for type I SHG) and KTP (for type II SHG) non-linear crystals to frequency double a CR beam at a fundamental frequency of $1064 \mathrm{~nm}$. In Section 8.3 it is discussed the transverse intensity patterns obtained at the focal plane of the system. We show that for type I the second harmonic intensity pattern is a light ring with a point of null intensity, while for type II the light ring possesses two dark regions. The experimental results are in good agreement with the proposed theoretical model. The beam evolution of the SHG CR beams are reported in Section 8.4, showing great similarities with the $\mathrm{CR}$ beam at the fundamental frequency. We discuss SHG CR beams generated at different positions of the non-linear crystals along the fundamental CR beam in Section 8.5. Since the SHG process depends strongly on the input intensity distribution, transverse intensity patterns generated do not resemble the ones obtained at the focal plane. Finally, in Section [8.6 we outline the main conclusions that can be drawn from our work.

The research contained in this chapter has been performed in collaboration of José Trull and Crina Cojocaru from the Universitat Politècnica de Catalunya (UPC) and published in Ref. [223]. 


\subsection{Introduction}

The invention of the laser opened many new areas of physics. In particular, the interaction of a powerful, monochromatic and coherent light source with optical media resulted on the born of non-linear optics. The first non-linear optical effect reported was second harmonic generation (SHG), in which two photons at frequency $\nu$ combine each other to form a single photon at frequency $2 \nu$. Note that in this process both energy $(E)$ and momentum $(\mathbf{k})$ are conserved:

$$
\begin{array}{r}
E_{f}=\hbar 2 \nu=\hbar \nu+\hbar \nu, \\
\mathbf{k}_{\mathbf{f}}=\mathbf{k}_{\mathbf{2} \nu}=\mathbf{k}_{\nu, \mathbf{1}}+\mathbf{k}_{\nu, \mathbf{2}} .
\end{array}
$$

Eq. (9.2) is also known as the phase-matching condition. Since linear momentum is a vectorial magnitude, both the direction and magnitude of this quantity must be preserved. In the simplest case the refractive indices at the two involved frequencies satisfy $n(2 \nu)=n(\nu)$ and we have collinear phase-matching, i.e., $\mathbf{k}_{\mathbf{2} \nu} \| \mathbf{k}_{\nu}$. For $n(2 \nu) \neq n(\nu)$, the phase matching condition implies, in general, non-collinear SHG.

In spite of being a relatively old phenomenon, only few articles have addressed CR in the non-linear regime [224 231, being all of them centered in the study of second harmonic generation (SHG) processes. In most of these works [224, 229], CR and its SH signal were generated in the same biaxial crystal (BC). This configuration ensures a very compact set-up, but unfortunately the phase matching direction does not coincide, in general, with one of the the optic axis of the crystal. Therefore, only materials with very large non-linearities are able to simultaneously generate $\mathrm{SH}$ and $\mathrm{CR}$. An alternative study of SHG in combination with CR can be carried out by placing a NLC after a $\mathrm{BC}[223,230]$. This configuration allows the adjustment of the phase matching condition for SHG in the NLC with the optic axis of the BC. In this case, a more efficient SHG process can be found. In what follows we present our results of SHG in type I (LBO) and type II (KTP) NLCs of a beam transformed by the CR phenomenon, that have in published in Ref. [223]. [223 231, being all of them centered in the study of second harmonic generation (SHG) processes. In most of these works [224, 229], CR and its SH signal were generated in the same biaxial crystal (BC). This configuration ensures a very compact set-up, but unfortunately the phase matching direction does not coincide, in general, with one of the the optic axis of the crystal. Therefore, only materials with very large non-linearities are able to simultaneously generate $\mathrm{SH}$ and CR. An alternative study of SHG in combination with CR can be carried out by placing a NLC after a BC [223, 230]. This configuration allows the adjustment of the phase matching condition for SHG in the NLC with the optic axis of the BC. In this case, a more efficient SHG process can be found. In what follows we present our results of SHG in type I (LBO) and type II (KTP) NLCs of a beam transformed by the CR phenomenon [223]. 


\subsection{Experimental set-up}

Fig. 8.1 shows in detail our experimental set-up.

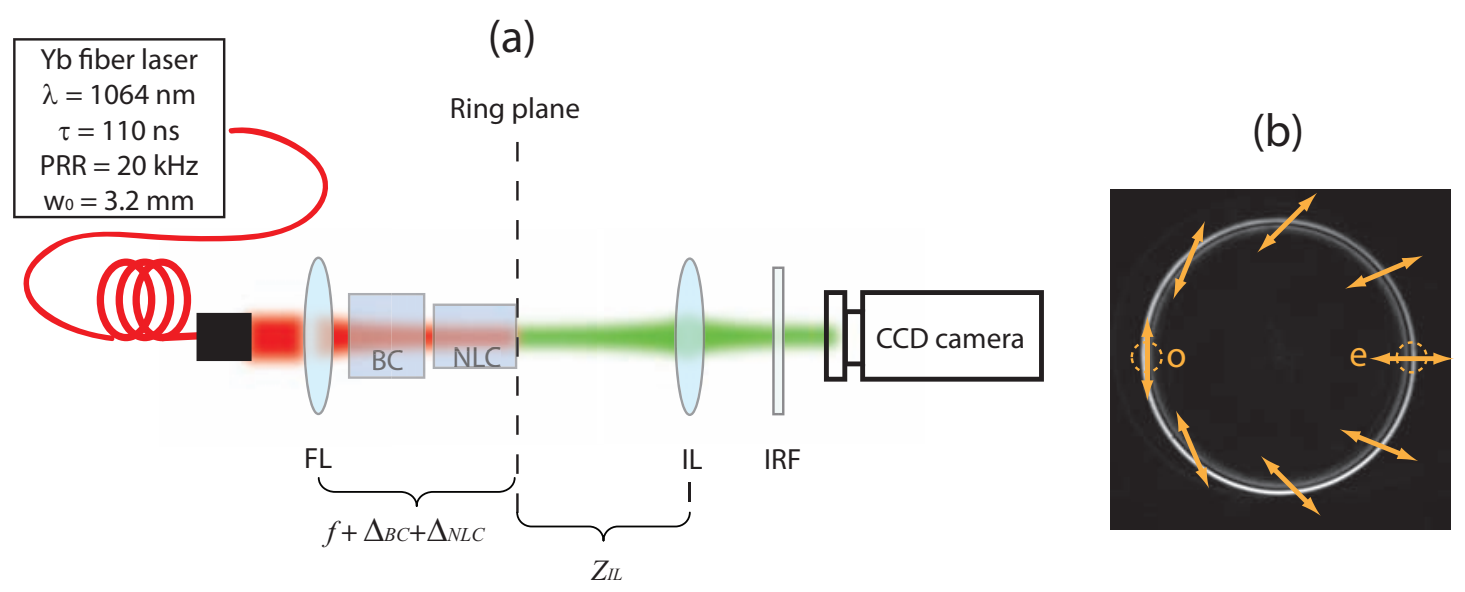

Figure 8.1: (a) Experimental set-up. A randomly polarized input beam with a beam waist radius of $w_{0}=3.2 \mathrm{~mm}$ is obtained from an $\mathrm{Yb}$ fiber laser generating light pulses at $1064 \mathrm{~nm}$ with pulse duration $\tau=(110 \pm 10) \mathrm{ns}$ at $20 \mathrm{kHz}$ repetition rate (PRR) and up to $10 \mathrm{~W}$ of nominal power per pulse. This beam is focused by a lens (FL) of $400 \mathrm{~mm}$ focal length to a $\mathrm{KGd}\left(\mathrm{WO}_{4}\right)_{2} \mathrm{BC}$ of length $L=28 \mathrm{~mm}$ and conicity, yielding $R_{0}=475 \mu \mathrm{m}$. At the ring plane, we place the NLCs: LBO (type I, $d_{\text {eff }}=0.668 \mathrm{pm} / \mathrm{V}$, $L_{\mathrm{LBO}}=10 \mathrm{~mm}$ ) and KTP (type II, $d_{\mathrm{eff}}=3.2598 \mathrm{pm} / \mathrm{V}, L_{\mathrm{KTP}}=8 \mathrm{~mm}$ ). The imaging lens IL projects different planes of the SHG propagated beams onto the CCD camera. The infrared filter (IRF) eliminates the radiation at the FH. $\Delta_{\mathrm{BC}}=L\left(1-1 / n_{\mathrm{BC}}\right)$ and $\Delta_{\mathrm{NLC}}=L_{\mathrm{NLC}}\left(1-1 / n_{\mathrm{NLC}}\right)$ are the longitudinal shift of the ring plane added by the $\mathrm{BC}$ and the NLC, respectively. (b) CR ring at the ring plane with the fine Poggendorff splitting obtained in the absence of the NLC. Double orange arrows show the polarization distribution along the ring. $o$ and $e$ denote the points with ordinary and extraordinary polarizations, respectively.

A randomly polarized input beam at $1064 \mathrm{~nm}$ is focused to a $\mathrm{KGd}\left(\mathrm{WO}_{4}\right)_{2} \mathrm{BC}$ under $\mathrm{CR}$ conditions. At the ring plane, where the $\mathrm{CR}$ ring at fundamental harmonic $(\mathrm{FH})$ appears, we place the NLCs oriented precisely under phase-matching conditions for optimal generation of SH. The focusing lens used ensures operation under plane wave approximation, i.e., $L_{\mathrm{NLC}} \approx z_{R}$ so that the NLC generates $\mathrm{SH}$ only from a unique transverse pattern of the CR beam. The length of the NLCs $\left(L_{\mathrm{LBO}}=10 \mathrm{~mm}, L_{\mathrm{KTP}}=\right.$ $8 \mathrm{~mm})$ is smaller than the distance of the Raman spot from the ring plane: $Z_{\text {Raman }} \approx$ $166 \mathrm{~mm}$. Finally, an imaging lens (IL, with position $\mathrm{Z}_{\mathrm{IL}}$ ) of $200 \mathrm{~mm}$ focal length projects the ring plane into the CCD camera. 


\subsection{Transverse intensity patterns at the focal plane}

Fig. 8.2(b) and Fig. 8.2(c) show the experimental SHG intensity patterns for type I and type II NLCs, respectively. We observe that in type I SHG, the transverse pattern consists of a light ring with a point of null intensity, resembling the pattern obtained in $\mathrm{CR}$ with linearly polarized beams [33. However, in this case the whole SH ring is linearly polarized with the polarization plane coinciding with the extraordinary polarization of the NLC. For type I NLC, SHG occurs in the form oo $\rightarrow e$. Thus, the point of the CR ring at the $\mathrm{FH}$ with polarization coinciding with the extraordinary mode of the NLC does not lead to SHG and, therefore, the resulting pattern in this case forms a crescent ring. With respect to type II SHG, we observe a light ring with two diagonally opposite points of null intensity. In this case, the ring is also linearly polarized coinciding with the extraordinary polarization of the NLC. Note that in type II SHG the doubling frequency process occurs through the channels $o e \rightarrow e$ and $e o \rightarrow e$. As a consequence, the two points of the $\mathrm{CR}$ ring at the $\mathrm{FH}$ with only ordinary or extraordinary polarization do not contribute to $\mathrm{SH}$ while the maximum $\mathrm{SH}$ intensity comes from those points of the $\mathrm{FH}$ ring with an equal contribution of ordinary and extraordinary polarizations.

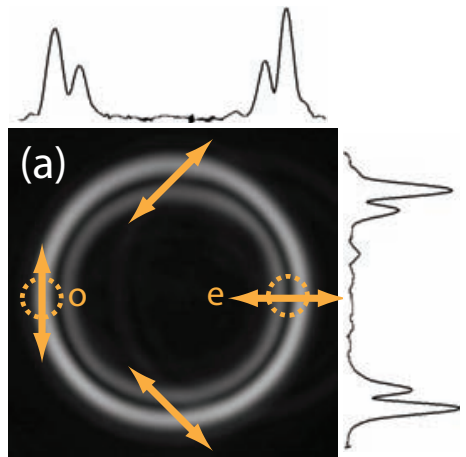

$\mathrm{FH}$

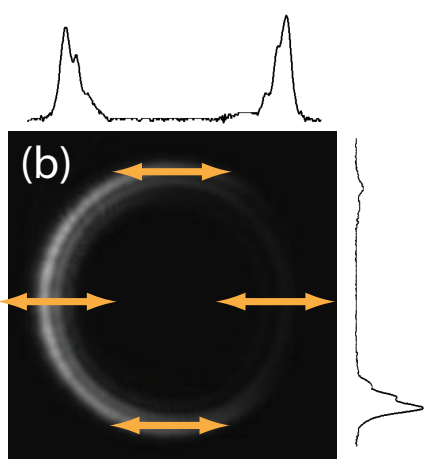

Type I (LBO)

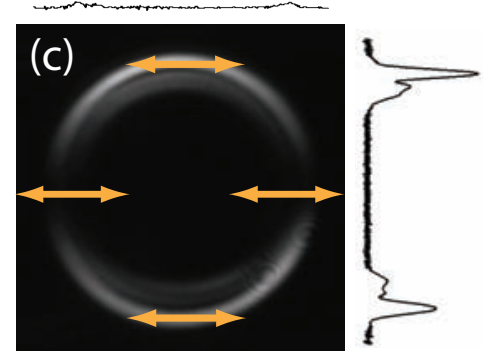

Type II (KTP)

Figure 8.2: Patterns of the FH (a), type I (b) and type II SH (c) generated with the NLCs placed at the ring plane. Patterns were captured by using the lens IL, see Fig. 8.1, to image the ring plane onto the CCD. Top and right insets are, respectively, the horizontal and vertical intensity profiles at the center of the images. Orange double arrows indicate the polarization plane.

Fig. 8.4 presents the SHG transverse intensity patterns for different CR rings obtained from biaxial crystals with corresponding different length. The $\mathrm{KGd}\left(\mathrm{WO}_{4}\right)_{2}$ crystals used in this case were $28 \mathrm{~mm}, 23 \mathrm{~mm}$ and $11 \mathrm{~mm}$ long $(476 \mu \mathrm{m}, 397 \mu \mathrm{m}$ and $181 \mu \mathrm{m}$ of ring radius respectively). Insets are the profile of the fundamental frequency. Since the state of polarization and transverse intensity pattern of the $\mathrm{FH}$ is well preserved even for the shorter BC, the results obtained for the three crystals are identical. 


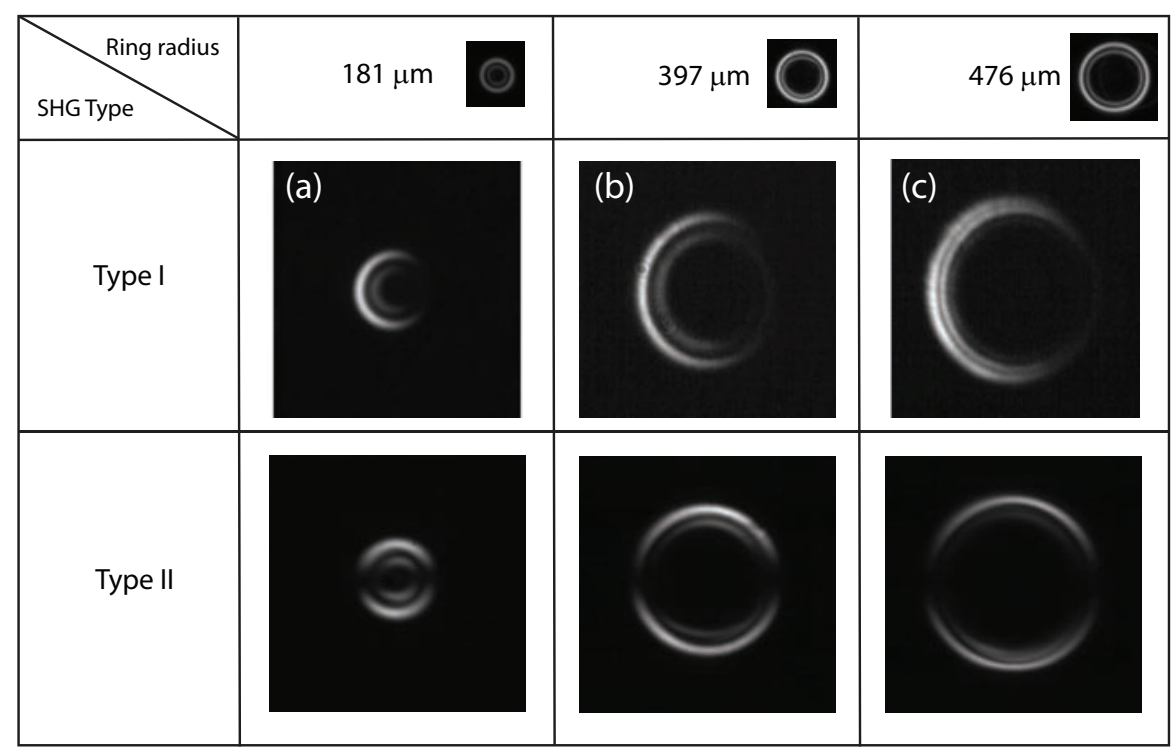

Figure 8.3: Type I (a)-(c) and type II (d)-(f) SHG patterns of conically refracted beams with ring radius of $181 \mu \mathrm{m}$ (second column), $397 \mu \mathrm{m}$ (third column), and $476 \mu \mathrm{m}$ (fourth column). Insets in first row represent in scale the rings at the fundamental frequency.

To obtain a quantitative description of the SH process we use the diffractive theory of CR presented in Chapter 2. In particular, it can be shown, that for the asymptotic case $\rho_{0} \rightarrow \infty$, the intensity of the CR beam at the ring plane is described by:

$$
|f(\xi, Z)|=\frac{e^{-\frac{\xi^{2}}{2 w_{Z}}}}{2^{5 / 4} w_{Z}^{3 / 4}} D_{\frac{1}{2}}\left(\frac{\sqrt{2} \xi}{\sqrt{w_{Z}}}\right),
$$

where $w_{Z}=1+i Z$ and $D_{\frac{1}{2}}(x)$ is the parabolic cylinder (Weber) function [29]. $\xi \equiv \rho-\rho_{0}$ is the normalized radial component in cylindrical coordinates. Taking into account both the polarization and the intensity distribution of the FH given by Eq. (8.3) and the the nature of the SHG processes (oo $\rightarrow e$ for type I; oe $\rightarrow e$ and $e o \rightarrow e$ for type II), it is straightforward to derive the corresponding analytical expressions for the $\mathrm{SH}$ intensity patterns from the CR ring:

$$
\begin{array}{r}
I_{\text {Type I }}=I_{2 \omega_{0}} \frac{|f(\xi, \eta)|^{4}}{\rho_{0}^{2}} \cos ^{4}\left(\frac{\varphi+\phi_{0}}{2}\right), \\
I_{\text {Type II }}=I_{2 \omega_{0}} \frac{|f(\xi, \eta)|^{4}}{\rho_{0}^{2}} \sin ^{2}\left(\varphi+\phi_{0}\right), \\
I_{2 \omega_{0}}=\left(\frac{P}{\lambda_{1} L \epsilon_{0} c} \frac{n_{1}}{n_{2}}\right) \tanh ^{2}\left[\sqrt{\left.\left(\frac{16 \pi^{2} d_{e f f}^{2} P L}{n_{1} n_{2} \epsilon_{0} c \lambda_{1}^{3}}\right)\right],}\right.
\end{array}
$$

where $\varphi$ indicates the point of the $\mathrm{CR}$ ring, $\phi_{0}$ is the mutual orientation between the planes of optic axes of the $\mathrm{BC}$ and the NLC and $\rho_{0} \equiv \frac{R_{0}}{w_{0}}$ measures the ring radius in 
beam waists. $I_{2 \omega_{0}}$ is the normalized intensity of the SH signal at the exit of a NLC for a Gaussian beam [232, where $P$ is the power of the input beam, $\lambda_{1}$ is the wavelength of the fundamental wave, $\epsilon_{0}$ is the vacuum permeability, $c$ is the speed of light in the vacuum, $d_{\text {eff }}$ is the maximum value of the effective $\chi^{2}$ coefficient and $n_{1}$ and $n_{2}$ are the optical refractive indexes of the NLC. In Fig. 8.4 we plot the azimuthal intensity variations obtained experimentally (symbols) and the corresponding theoretical solutions (solid lines) for type I (red) and type II (black) NLCs.

Note, in addition, that the inner Poggendorff ring is almost non visible in the SHG intensity patterns, see Figs. 8.2(b) and 8.2(c). For the FH, it has been shown that the intensity of the input beam redistributes between the two Poggendorff rings in a ratio 3:1 (outer:inner) [27]. Since $I_{2 \omega_{0}} \propto I_{\omega_{0}}^{2}$, the two Poggendorff rings do not generate the same SH intensity signal, being the SHG outer ring much more intense than the inner one.

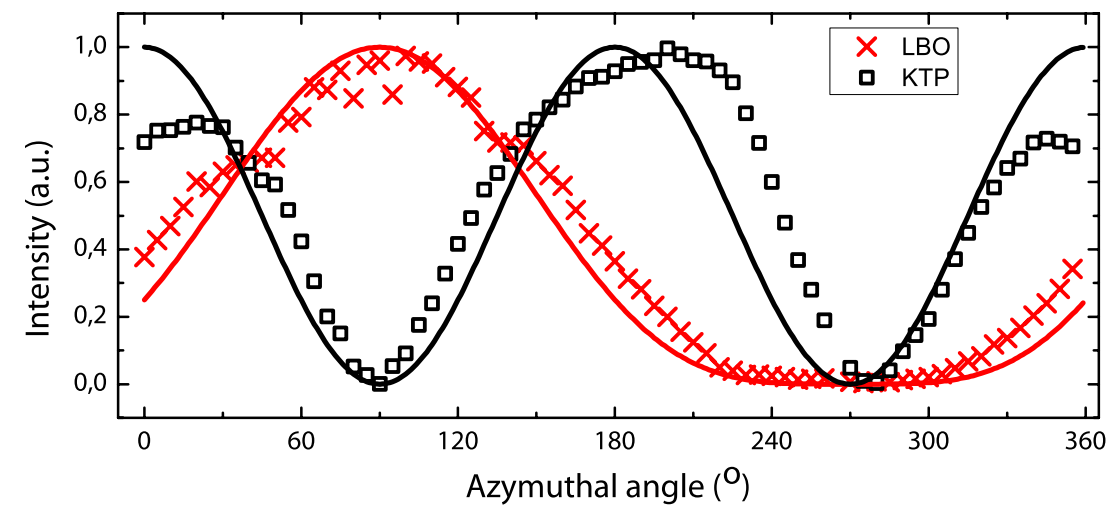

Figure 8.4: Azimuthal intensity distribution of the final patterns for type I (LBO) and type II (KTP) SHG. Symbols represent the experimental data, while solid lines are the corresponding analytical solutions from Eqs. (8.4)-(8.6).

\subsection{Beam evolution of the SHG beams}

Fig. 8.5 presents the evolution of the transverse intensity patterns of the SHG beams obtained by imaging different planes along the beam propagation. Comparing with the intensity patterns observed in the absence of the NLC, i.e., under conditions of CR (top row), one concludes that the frequency doubled waves are also CR beams, being their evolution completely analogous to the FH. We have observed two focusing spots placed symmetrically from the ring plane of the $\mathrm{SH}$ signals resembling the Raman spots of CR. This behavior is expected since SHG is a non-linear process that converts both the intensity and the phase of the incoming wavefront inside the NLC. 

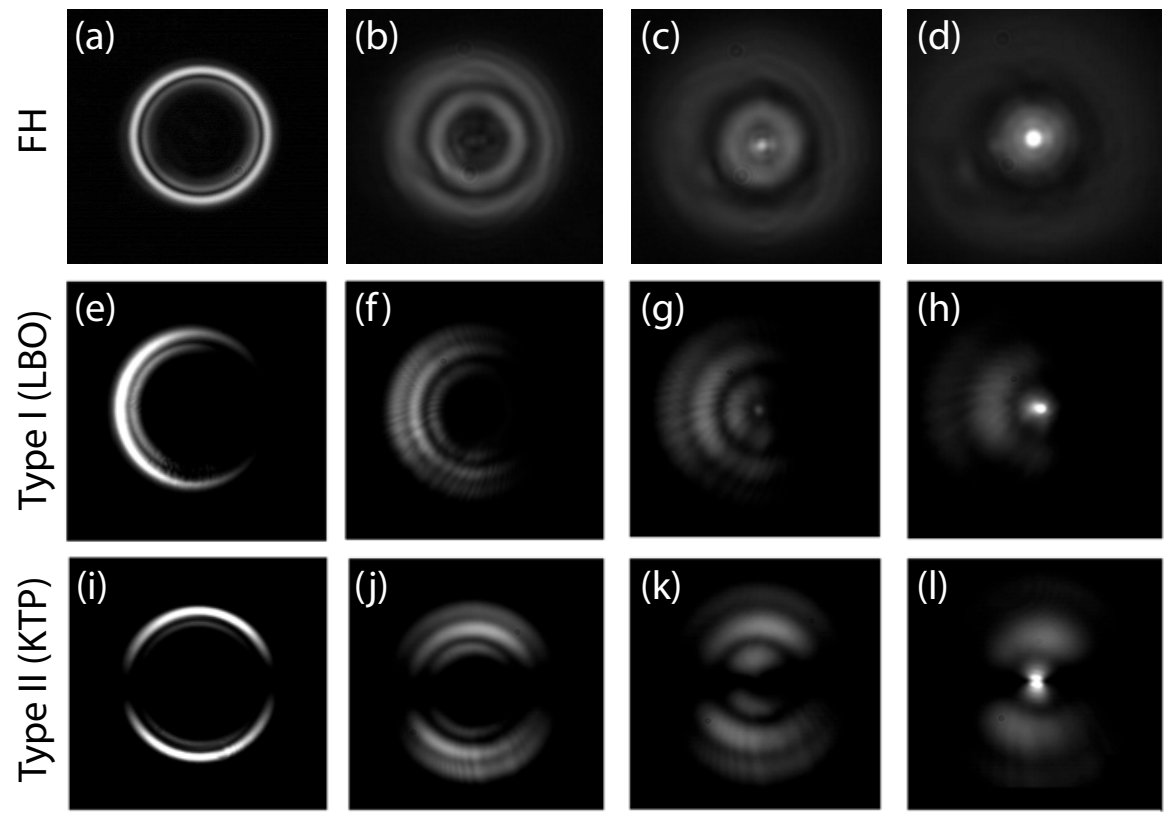

Figure 8.5: Evolution of the transverse intensity profile in type I (middle row) and type II (bottom row) SHG when the NLCs are placed at the ring plane of the CR beam. The extraordinary polarization in the NLC was perpendicular to the plane of optic axes of the BC, i.e., $\phi_{0}=0^{\circ}$. We note that the Raman-like spots for second harmonic, see (d) and (h), have been observed on both sides from the ring plane. First row images show the transverse intensity patterns observed in the absence of the NLC.

\subsection{Influence of the position of the non-linear crystal}

It is well known that SHG depends on the beam's profile passing through a NLC. To illustrate how is this dependence for CR beams, power measurements of the total SHG intensity were taken with the NLCs placed at different positions of the CR beam. Both in type I and type II SHG, the Raman spot is the most efficient plane. The corresponding transverse intensity patterns obtained at the focal image plane when the NLCs are placed at different positions of the beam propagation at FH are shown in Fig. 8.5(a)-(h). It is particularly interesting that, when the NLCs are placed out of the ring plane, there are contributions to the SHG beam of all the original CR beam, i.e., of the CR ring, the Raman spot and the secondary rings at the same time. This has been also pointed out by Peet and Shchemelyov [230].

\subsection{Conclusions}

In summary, we have reported SHG in type I and type II NLCs from an input beam refracted conically after passing along the optic axis of a BC. This configuration allows 
aligning precisely the phase-matching direction of the NLC. For type I, the SH pattern at the ring plane forms a light ring with a point of null intensity, corresponding to the extraordinary polarization of the FH. In contrast, for type II SHG, the light ring possesses two dark points that correspond to the two points of the FH with only ordinary or extraordinary polarizations. We have provided a qualitative explanation of the $\mathrm{SH}$ intensity patterns in terms of the different channels that contribute to the SH signal and derived an analytical solution that is in good agreement with the experimental results. Besides, we have investigated the spatial evolution of the SH beams showing that they resemble conically refracted beams. Finally, we have shown that the Raman spot is the most efficient region for SHG and demosntrated that when the NLCs are placed out of the ring plane, the transverse intensity patterns observed at the focal image plane posses contributions from the $\mathrm{CR}$ rings and the Raman spot at the same time.
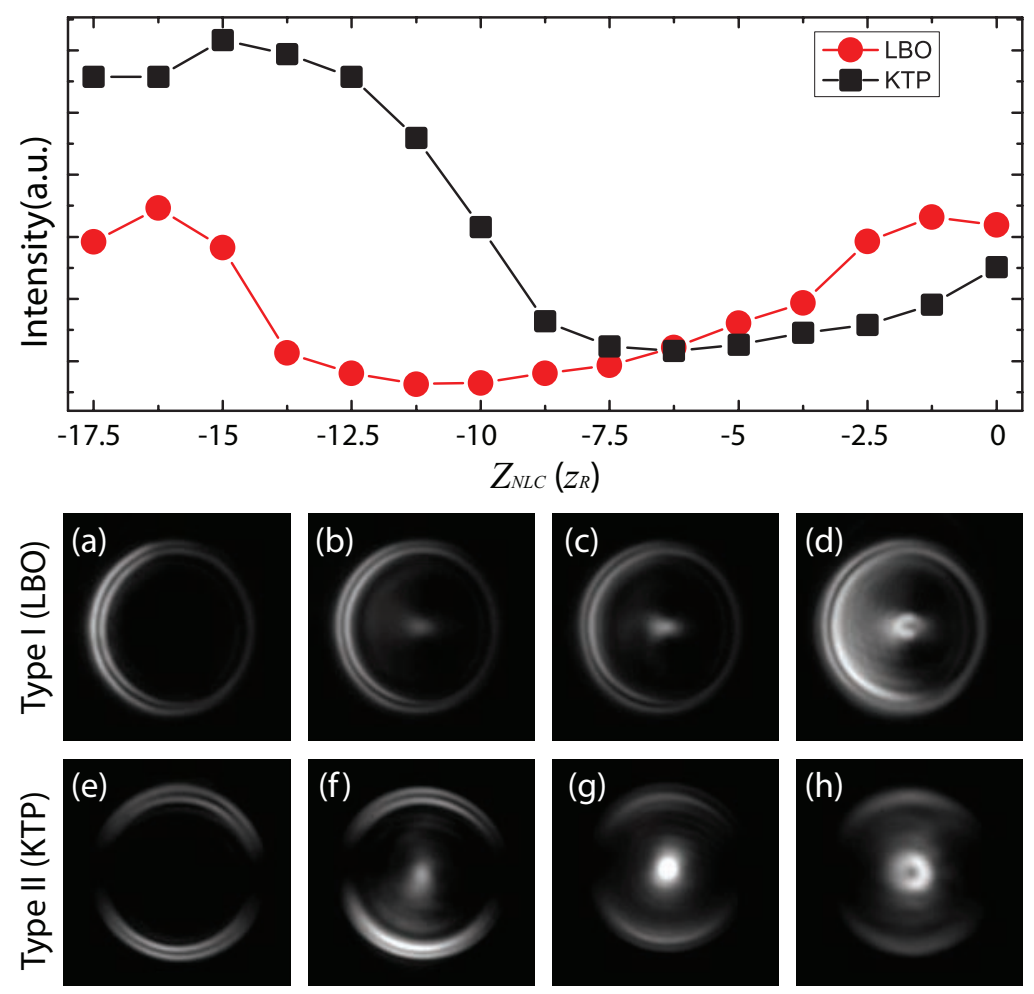

Figure 8.6: Top plot: Measured total output SHG power for different positions of the NLCs along the FH beam propagation. (a)-(h) Patterns obtained as the position of the type I (first row) and type II (bottom row) NLC is placed at different positions along the beam propagation direction of the FH beam. Subsequent images are separated a distance of $5 z_{R}$. 
CHAPTER 9

Testing quantum mechanics with conical refraction

In this Chapter there are presented, to our knowledge, the first attempts to use CR for quantum optical applications. We propose the use of CR to measure linear momentum entanglement between two twin photons generated by means of spontaneous parametric down-conversion through a polarization measurement. This proposal was conceived during a research stay at the group of Prof. Miles Padgett (University of Glasgow). Unfortunately, the duration of the stay was insufficient to achieve good quality experimental data, reason for which we will restrict to the theoretical proposal. The Chapter is organized as follows. In Sec. 9.1 we introduce the Einstein-PodolskyRosen paradox and Bell inequalities, that are at the heart of our proposals. Sec. 9.2 is devoted to explain the transformation of a spontaneous parametric down-conversion beam by means of the conical refraction phenomenon. Then, in Sec. 9.3 we show how a single biaxial crystal can be used to swap linear momentum entanglement for polarization entanglement. We present the quantum state of the system and how it can be converted into a polarization state. To conclude, in Sec. 9.4 we sum up the ideas presented in this Chapter and discuss the reasons why we did not succeed with the experiments.

\subsection{Introduction}

In 1935 Albert Einstein, Boris Podolsky and Nathan Rosen presented a paradox (the EPR paradox) that became the major critique against the Copenhagen interpretation of quantum mechanics [233. With that critique, EPR aimed to show that the wavefunction is not enough to describe reality and, therefore, that the quantum-mechanical description of the world is incomplete. The EPR paradox can be summarized as follows. Let's consider a disintegration process that emits a pair of particles and let them travel 
a time enough so they are space-time separated but they are entangled in momentum and position. Quantum mechanics affirms that there are some quantities (observables) that cannot be simultaneously measured with complete precision. In this sense, it is stated that operators associated to these conjugated observables. This is the case of momentum $\mathbf{p}$ and position $\mathbf{r}$, whose canonical commutation relation leads to the well known uncertainty principle $\sigma_{x} \sigma_{p} \geq \hbar / 2$. Besides the fact that the uncertainty principle introduces some constrains, there is no restriction to measure simultaneously the momentum of one of a pair of particles and the position of the other one. However, such measurement would give us a complete knowledge of both the position and momentum of the two particles, since in the disintegration process both momentum and center of mass position must be preserved. This seems to be in contradiction with the Copenhagen interpretation of quantum mechanics. EPR stated that a more fundamental theory based on some unknown (hidden) variables should be developed to make quantum mechanics complete. Soon afterward, Neils Bohr wrote a reply to the EPR paradox where he stated that it is an error to consider both particles as two separate systems [234. After the particles have interacted once, they must be considered as part of a single system. This response to the EPR paradox was enough for the quantum-mechanical defenders but it was not enough to discard the existence of some hidden variable theory that explained the oddities of quantum mechanics.

In 1964 John S. Bell published a work that settle any discussion on that sense [235,236]. Bell realized that EPR assumed that the measuring process performed in one particle is not affecting the measuring process carried out over the other particle, i.e., the measurements are local. By taking the latter statement and the idea of the EPR paradox that separated particles have separated physical realities as axioms, Bell derived a set of inequalities that every local theory of hidden variables should satisfy. To the delight of quantum mechanical backers, he found that in some cases quantum mechanics does not satisfy those inequalities and, therefore, that quantum mechanics cannot be described by any local theory of supplemental variables. The direct consequence of this finding is that quantum mechanics is non-local, at variance with any intuition that we have of our everyday world.

The problem is that situations of conflict where quantum mechanics violate Bell inequalities are rare and hard to test experimentally, since a very well defined entangled state and the corresponding measuring devices are needed. In 1969, John F. Clauser, Michael A. Horne, Abner Shimony and Richard A. Holt proposed a feasible experiment to test Bell inequalities based on polarization entangled correlated photons produced in certain atomic cascades that opened the route to a first batch of experiments [237-240]. With the exception of Ref. [238] all these works demonstrated agreement with quantum mechanics, i.e., violation of Bell inequalities. However, in these first experiments the detection efficiency was so low that one additional assumption was required: given a pair of photons emerging from the polarizers used, the probability of their joint detection is independent of the polarizer orientations. Alain Aspect and co-workers were able to 
avoid the latter assumption by using a very efficient atomic cascade process that enabled to clearly violate Bell inequalities [241 243].

Nowadays, most experiments devoted to further test Bell inequalities take profit of the non-linear optical phenomenon of spontaneous parametric down-conversion (SPDC) that in 1995 was demonstrated to be an efficient source of polarization entangled photon pairs by Anton Zeilinger and co-workers [244]. In fact, this source also produces frequency entangled [245,247] and both linear [248,249] and orbital angular momentum entangled [250 255] photon pairs. In our case, we will use photon pairs produced by SPDC to propose a novel method to swap linear momentum entanglement into polarization entanglement by means of CR. Additionally, we will propose a CR-based Bell-like inequality to test entanglement between the linear momentum and the polarization of a single photon.

\subsection{Conical refraction of a spontaneous parametric down- converted beam}

(a)

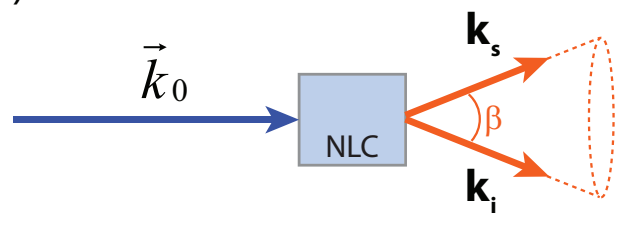

(b)
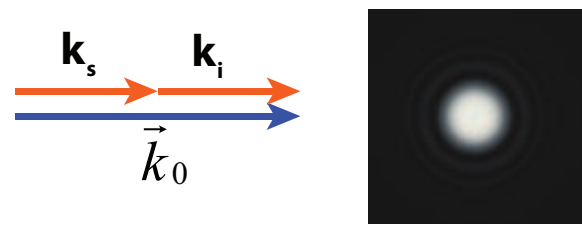

(c)
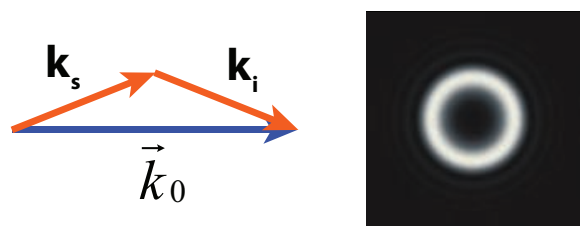

Figure 9.1: (a) Spontaneous parametric down-conversion (SPDC) process: a pump photon of frequency $\nu_{0}$ and momentum $\left|\mathbf{k}_{\nu_{0}}\right|=2 \pi n / \lambda_{0}$ decays into two photons with corresponding momentum $\left|\mathbf{k}_{\nu_{j}}\right|=2 \pi n / \lambda_{j}(j=\{s, i\})$. (b) Perfect collinear phase-matching: the two photons are emitted parallel to the direction of the pump and the down-converted light forms a bright spot. (c) Perfect non-collinear phase-matching: the signal and idler photons have opposite transverse momentum and their directions belong to a cone. In this case the transverse light pattern of the down-converted light forms a light central ring. NLC: nonlinear crystal. 
Spontaneous parametric down-conversion (SPDC) is a non-linear process in which a single photon decays into two new photons called as idler and signal photons. In this process, which was firstly identified by Kleinman as optical parametric noise [256], both the energy and the linear momentum must be preserved, i.e.

$$
\begin{array}{r}
h \nu_{0}=h \nu_{s}+h \nu_{i}, \\
\mathbf{k}_{\nu_{0}}=\mathbf{k}_{\nu_{s}}+\mathbf{k}_{\nu_{i}},
\end{array}
$$

being Eq. (9.1) and Eq. (9.2) known as the phase-matching conditions. If $\left|\mathbf{k}_{\nu_{0}}\right|=$ $\left|\mathbf{k}_{\nu_{s}}\right|+\left|\mathbf{k}_{\nu_{i}}\right|$ collinear phase-matching is found and the transverse intensity pattern of the SPDC beam at the output of the non-linear crystal forms a bright spot, see Fig. 9.1(b). In contrast, if the previous relation does not hold, the non-collinear phase-matching condition produces a SPDC beam evolving as a cone whose transverse intensity pattern forms a light ring, as shown in Fig. 9.1(c). Note that in this case, the signal and idler photons appear at diametrically opposite positions on the light ring.

The signal and idler photons generated in the SPDC process have been reported to be entangled in many different degrees of freedom such as polarization [244, 257, frequency [258], linear momentum [259], transverse position [260] and orbital angular momentum [250]. In our case, we use a BBO uniaxial crystal to produce type-I SPDC photons with the same polarization and frequency and we pay attention to correlations in linear momentum. The intensity profile of the SPDC beam in the far-field of the crystal imaged with a lens of focal length $f$ can be modeled through the following expression:

$$
I(r)_{\mathrm{SPDC}}=\operatorname{sinc}^{2}\left(\frac{a r^{2}}{f}+\alpha\right)
$$

where $\alpha=\left(\left|\mathbf{k}_{\nu_{0}}\right|-\left|\mathbf{k}_{\nu_{s}}\right|-\left|\mathbf{k}_{\nu_{i}}\right|\right) L / 2$ is a phase-matching parameter that determines the opening angle of the SPDC cone and $a=\left(\left|\mathbf{k}_{\nu_{s}}\right|+\left|\mathbf{k}_{\nu_{i}}\right|\right) L / 4 n^{2}$, where $n$ is the refractive index for the signal and idler wavelengths and $L$ is the crystal length [261. For our purposes, we require non-collinear phase-matching to obtain a clear SPDC light cone. The transformation of such a beam by means of CR can be easily predicted making use of Eqs. (2.12)-(2.15) and (9.3). Both the theoretical and the experimental transverse intensity patterns obtained when the SPDC beam at the far-field of the non-linear BBO crystal ( $\left.\alpha \approx-3, f=200 \mathrm{~mm}, L=5 \mathrm{~mm}, \lambda_{0}=355 \mathrm{~nm}, \lambda_{i}=\lambda_{s} \approx 2 \lambda_{0}\right)$ passes through a biaxial crystal are presented in Fig. 9.2. Note that the polarization of the SPDC beam was transformed into circular by means of a quarter wave-plate. The transverse intensity pattern is formed by a pair of concentric light rings without Poggendorff splitting. We have checked that the polarization distribution along the rings is CR-like, i.e., it is linear at every point with the azimuth rotating $\pi$ rad for a complete turn along the rings. In what follows, we take profit of the linear momentum entanglement provided by the SPDC process and of the linear momentum-polarization relation offered by the CR phenomenon to propose a new Bell-type inequality test of quantum mechanics. 

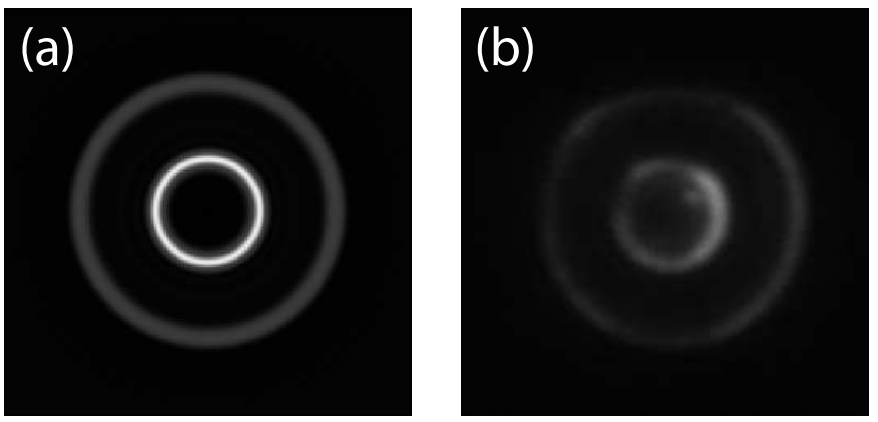

Figure 9.2: (a) Numerically calculated, by using Eq. (9.3), and (b) experimentally recorded transverse intensity pattern of the down-converted light cone after being transformed by the CR phenomenon. For the experimental image, a BBO non-linear crystal was used to down-convert input photons at $355 \mathrm{~nm}$ into momentum-entangled photon pairs at $710 \mathrm{~nm}$. Then, the optic axis of a KTP biaxial crystal $(l=10 \mathrm{~mm})$ was aligned with the beam propagation direction of the pump beam and a CCD camera was used to record the transverse intensity pattern. $\alpha \approx-5$.

\subsection{Detection of linear momentum entanglement with con- ical refraction}

As commented in the previous section, SPDC can be used to generate photon pairs entangled in time, frequency, polarization, linear momentum and orbital angular momentum. For the case of linear momentum entangled photons, entanglement detection is not an easy task, since transverse positions must be measured, e.g., by using a single photon CCD camera [146, 260]. In contrast, detection of polarization entanglement is much simpler, since only linear polarizers and single photon detectors are needed. In this Section we propose how to take profit of the CR phenomenon to transform linear momentum entanglement into polarization entanglement.

We consider that the down-converted photons have the same frequency, i.e., that $\nu_{s}=\nu_{i}=\equiv \nu=\nu_{0}$ and also that they are generated in type I SPDC (therefore they are horizontally polarized). As previously stated, the signal and idler photons are, in general, emitted in a light cone, see Figs. 9.1(a) and (c). Their linear momentum $\mathbf{k}_{s, i}$ has both transverse $\mathbf{k}_{s, i}^{\perp}$ and parallel $\mathbf{k}_{s, i}^{\|}$components to the propagation direction of the fundamental beam. These momentum components can be written as

$$
\begin{aligned}
& \mathbf{k}_{s, i}^{\perp}=\frac{k_{0}}{2} \sin \beta\left(\cos \phi_{s, i} \mathbf{u}_{\mathbf{x}}+\sin \phi_{s, i} \mathbf{u}_{\mathbf{y}}\right), \\
& \mathbf{k}_{s, i}^{\|}=\frac{k_{0}}{2} \cos \beta \mathbf{u}_{\mathbf{z}},
\end{aligned}
$$

where $k_{0}=2 \pi n / \lambda_{0}$ is the wave-number of the fundamental photon, $\beta$ is the aperture angle of the down-converted cone, $\phi_{s, i}$ is the azimuthal angle in cylindrical coordinates 
and $\mathbf{u}_{\mathbf{x}, \mathbf{y}, \mathbf{z}}$ form an orthogonal basis of unitary vectors. If we look at the transverse momentum component and define $\phi_{s} \equiv \phi$, due to momentum conservation the relation $\phi_{i}=\phi+\pi$ must be satisfied. Fig. 9.3 shows our experimental proposal. The state of polarization of the down-converted photons emerging from the non-linear crystal is transformed by means of a quarter wave-plate into left-handed circular $\left(\sigma^{+}\right)$, for instance. The system formed by the down-converted photons can be mathematically described by the following state:

$$
|\psi\rangle=\frac{1}{2 \pi} \int_{0}^{2 \pi} d \phi\left|\phi, \sigma^{+}\right\rangle_{s}\left|\phi+\pi, \sigma^{+}\right\rangle_{i}
$$

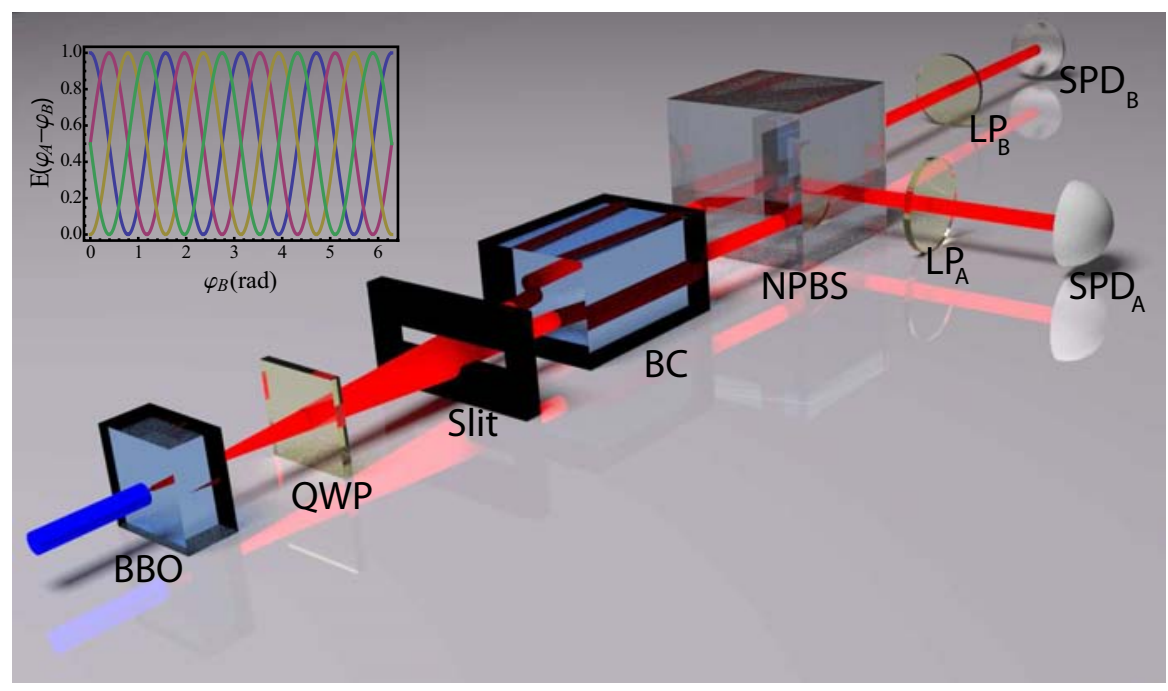

Figure 9.3: Proposed set-up to demonstrate linear momentum entanglement through a polarization measurement with CR. The state of polarization of the SPDC cone emitted by a BBO non-linear crystal is transformed from linear into circular by means of a quarter wave-plate (QWP). A slit selects only two azimuthal sectors of the light cone and, therefore, fixes the transverse momentum of the transmitted twin photons. A biaxial crystal $(\mathrm{BC})$ couples the transverse momentum of photons with a certain linear polarization that depends on the orientation of the BC. A non-polarizing beam splitter (NPBS) sends each photon to a linear polarizer (LP) and a single photon detector (SPD) to measure coincidences counts while rotating the mutual angle between the transmission axes of the polarizers. The inset shows different plots of the correlation function between the LPs of branches $A$ and $B$ as a function of the orientation of the transmission axis of $\operatorname{LP}_{B}\left(\varphi_{B}\right)$ calculated from Eq. (9.14) for $\varphi_{A}=0 \mathrm{rad}$ (blue line), $\varphi_{A}=\pi / 8 \mathrm{rad}$ (purple line), $\varphi_{A}=\pi / 4 \mathrm{rad}$ (yellow line) and $\varphi_{A}=3 \pi / 8 \mathrm{rad}$ (green line).

Then, a slit whose aperture plane is described by the azimuthal angle $\phi_{k}$ projects the state described by Eq. (9.6) into the state

$$
|\psi\rangle^{\prime}=\left|\phi_{k}, \sigma^{+}\right\rangle_{s}\left|\phi_{k}+\pi, \sigma^{+}\right\rangle_{i}
$$


which is a state with well defined transverse linear momentum given by the azimuthal angle $\phi_{k}$. This bi-photon state passes through a biaxial crystal with $\mathbf{k}_{s, i}^{\|}$parallel to one of its optic axis. Following the theoretical formalism used in Sec. 2.3.3 it is straightforward to deduce that the resulting state can be described by

$$
|\psi\rangle_{\text {out }}=\frac{1}{\sqrt{2}}\left(\left|\phi_{k}, \frac{\phi_{k}}{2}\right\rangle_{s}\left|\phi_{k}+\pi, \frac{\phi_{k}}{2}+\frac{\pi}{2}\right\rangle_{i}+\left|\phi_{k}+\pi, \frac{\phi_{k}}{2}+\frac{\pi}{2}\right\rangle_{s}\left|\phi_{k}, \frac{\phi_{k}}{2}\right\rangle_{i}\right),
$$

where we have set the orientation of the characteristic $\mathbf{G}$ vector of the biaxial crystal at $\varphi_{c}=0$. Note that the action of the biaxial crystal over the bi-photon state is twofold: (1) the linear momentum of each photon has equal probabilities of being projected at azimuthal angles $\phi_{k}$ and $\phi_{k}+\pi$ and (2) the state of polarization of each state is correspondingly transformed from circular to linear with azimuth $\phi_{k} / 2-\varphi_{c}$ and $\phi_{k} / 2+$ $\pi / 2-\varphi_{c}$. Without loosing generality we can set $\phi_{k}=0$, which leaves the state from Eq. (9.8) as

$$
|\psi\rangle_{\text {out }}=\frac{1}{\sqrt{2}}\left(|\rightarrow, H\rangle_{s}|\leftarrow, V\rangle_{i}+|\leftarrow, V\rangle_{s}|\rightarrow, H\rangle_{i}\right),
$$

where we have defined transverse linear momentum states with azimuthal angle $0 / \pi$ as $\rightarrow / \leftarrow$ and linearly polarized states with azimuth at $0 / \frac{\pi}{2} \mathrm{rad}$ as $H / V$. The combined role of the biaxial crystal and the NPBS is to remove the information on the linear momentum of each photon, so that the bi-photon state approaching the detectors is:

$$
|\psi\rangle_{\text {out }}=\frac{1}{\sqrt{2}}\left(|H\rangle_{s}|V\rangle_{i}+|V\rangle_{s}|H\rangle_{i}\right)
$$

which is a well known Bell-state for polarization entangled photons (see [236] and the references therein). Once the photons are spatially separated by the NPBS and passed through the corresponding linear polarizers $A$ and $B$ with respective transmission axis at angles $\varphi_{A}$ and $\varphi_{B}$, the expected quantum coincidence counts $C_{i j}$ (where $i, j \in 0,1$ ) at given single photon detectors after the linear polarizers are

$$
\begin{aligned}
& C_{00}\left(\varphi_{A}, \varphi_{B}\right)=C_{11}\left(\varphi_{A}, \varphi_{B}\right)=\frac{1}{2} \sin ^{2}\left(\varphi_{A}-\varphi_{B}\right), \\
& C_{01}\left(\varphi_{A}, \varphi_{B}\right)=C_{10}\left(\varphi_{A}, \varphi_{B}\right)=\frac{1}{2} \cos ^{2}\left(\varphi_{A}-\varphi_{B}\right) .
\end{aligned}
$$

For these coincidence counts, the well known correlation function

$$
E\left(\varphi_{A}, \varphi_{B}\right)=\frac{C_{00}\left(\varphi_{A}, \varphi_{B}\right)+C_{11}\left(\varphi_{A}, \varphi_{B}\right)-C_{01}\left(\varphi_{A}, \varphi_{B}\right)-C_{10}\left(\varphi_{A}, \varphi_{B}\right)}{C_{00}\left(\varphi_{A}, \varphi_{B}\right)+C_{11}\left(\varphi_{A}, \varphi_{B}\right)+C_{01}\left(\varphi_{A}, \varphi_{B}\right)+C_{10}\left(\varphi_{A}, \varphi_{B}\right)}
$$

reads:

$$
E\left(\varphi_{A}, \varphi_{B}\right)=\cos ^{2}\left[2\left(\varphi_{A}-\varphi_{B}\right)\right] .
$$

Therefore, the Bell parameter $S \equiv E\left(\varphi_{A}, \varphi_{B}\right)+E\left(\varphi_{A}^{\prime}, \varphi_{B}^{\prime}\right)+E\left(\varphi_{A}^{\prime}, \varphi_{B}\right)-E\left(\varphi_{A}, \varphi_{B}^{\prime}\right)$ reaches a maximum value of $2 \sqrt{2}$, which is the upper bounding for violation of Bell inequalities. 


\subsection{Conclusions}

In this Chapter we have proposed the use of CR to transform linear momentum entanglement of twin photons generated in a non-linear crystal by means of SPDC into polarization entanglement. It has been shown that the CR phenomenon couples a given transverse linear momentum with a certain linear polarization. We have described the quantum state of photons before and after the biaxial crystal in terms of these two observables and shown that, at least theoretically, linear momentum entanglement is transformed into polarization entanglement. It has been demonstrated that this output quantum state is a Bell state for polarization with a well known Bell inequality that gives a maximum value of $2 \sqrt{2}$ of the $S$ parameter.

Unfortunately, the experimental data collected from the experiments carried out in order to demonstrate our proposal did not have quality enough to measure the Bell $(S)$ parameter. The major drawback that we suffered during the research stay at the group of Prof. Miles Padgett was noise from the environment that strongly affected the bucket detectors used, together with the lack of extra time to keep on with the experiment.

In case of being demonstrated experimentally, our proposal would open a new route to demonstrate linear momentum entanglement of twin photons, which is a hard task due to the high sensibility of the CCD detectors that are needed, with a relatively simple polarization measurement. Note that, as commented in previous Chapters, CR is a linear effect that does not depend on the intensity of the input source. Additionally, biaxial crystals can be transparent to a high spectral range and they convert the full power of the input light source, which would significantly decrease losses compared with other systems. 
CHAPTER 10

\section{Conical refraction for free-space optical communications}

In this Chapter, we propose a polarization de-multiplexing and multiplexing system based on conical refraction to increase the channel capacity for free-space optical communication applications. The proposed technique is based on the forward-backward optical transform occurring when a light beam propagates consecutively along the optic axes of two identical biaxial crystals with opposite orientations of their conical refraction characteristic vectors. We present an experimental proof of usefulness of the presented technique by increasing in one order of magnitude the channel capacity at optical frequencies in a propagation distance of $4 \mathrm{~m}$. The Chapter is organized as follows. In Sec. 10.1 we discuss different FSOC techniques that can be found in the literature. Then, the forward-backward CR optical transform that is at the heart of our FSOC system is presented in Sec. 10.2. Sec. 10.3 is devoted to show our experimental proposal, in which we use three biaxial crystals, and the corresponding experimental results. Finally, in Sec. 10.4 we summarize our work and envisage future possible experiments based on our technique.

The research contained in this chapter has been published in Ref. [82] and patented in Ref. 262].

\subsection{Introduction}

In optical communications, different properties of a light field, such as its intensity, wavelength, polarization, and orbital angular momentum (OAM), can be used to provide optical signals to efficiently transmit the information so that these signals do not interfere each other along the communication channel. Thus, for example, the capacity of a communication channel can be substantially increased if one multiplexes different 
wavelengths of various input optical carrier signals into a single channel by using the Wavelength Division Multiplexing (WDM) technique [263]. For a monochromatic laser beam, Laguerre-Gaussian light beams carrying orbital angular momentum (OAM) in the helicity of their phase fronts have been proposed [132] as a basis of carrier signals allowing, in principle, for an arbitrary increase of the channel capacity [264 267]. However, there are practical drawbacks that restrict the range of applicability of the OAM encoding technique [268, 269] such as the large divergence of high order OAM modes, which prevent their use for free space optical communications (FSOC) at long distances.

Alternatively, also for a monochromatic field, one could additionally use the polarization degree of freedom of a light beam as a carrier basis of signals for FSOC links. In this case, nevertheless, the use of a polarization beam splitter allows, at most, to double the FSOC channel capacity. In this Chapter, we present a novel method to de-multiplex and multiplex a monochromatic input light beam into, in principle, an arbitrary large number of polarization states by means of the CR phenomenon. Our formalism is closely related to the wave-vector and polarization formalism presented in Sec. 2.3 and on the forward-backward optical transformation carried out by the CR phenomenon in a cascade of two biaxial crystals with opposite orientations of their $\mathbf{G}$ vectors.

\subsection{The forward-backward conical refraction transforma- tion}

As it has been previously shown, when an ideally collimated circularly polarized light beam passes along the optic axis of a biaxial crystal it refracts conically inside the crystal and emerges as a collimated hollow cylinder whose transverse intensity pattern forms a light ring. This light ring is laterally shifted being both the direction of the displacement as well as the ring radius given by the so-called characteristic $\mathbf{G}$ vector of the biaxial crystal [33, see Fig. 2.1. As a consequence, a crystal with orientation of its $\mathbf{G}$ vector $\varphi_{G}=90^{\circ}$ shifts the center of the CR ring a distance $R_{0}$ in the $+y$ direction, where we have considered that $\mathbf{G}$ is contained in the $x y$ plane and beam propagation parallel to the $z$ direction, as sketched in Fig. 10.1(a). In contrast, a crystal with orientation of its $\mathbf{G}$ vector $\varphi_{G}=270^{\circ}$, i.e., opposite to the previous case, shifts the $\mathrm{CR}$ ring a distance $R_{0}$ in the $-y$ direction, see Fig. 10.1(b). If both effects are combined, the output beam at the exit of the second biaxial crystal is identical to the input beam entering into the first biaxial crystal. In other words, the birefringent effects of both biaxial crystals cancel each other and the input beam remains invariant after passing through the 2-crystal cascade. This particular arrangement, which allows for an optical CR forward-backward transform, will be called in what follows as the degenerate 2-crystal cascade CR configuration. Note that in this simplified explanation, we have only considered ideally collimated beams. However, as we will demonstrate in what follows, the here defined forward-backward transform also applies to focused beams. 

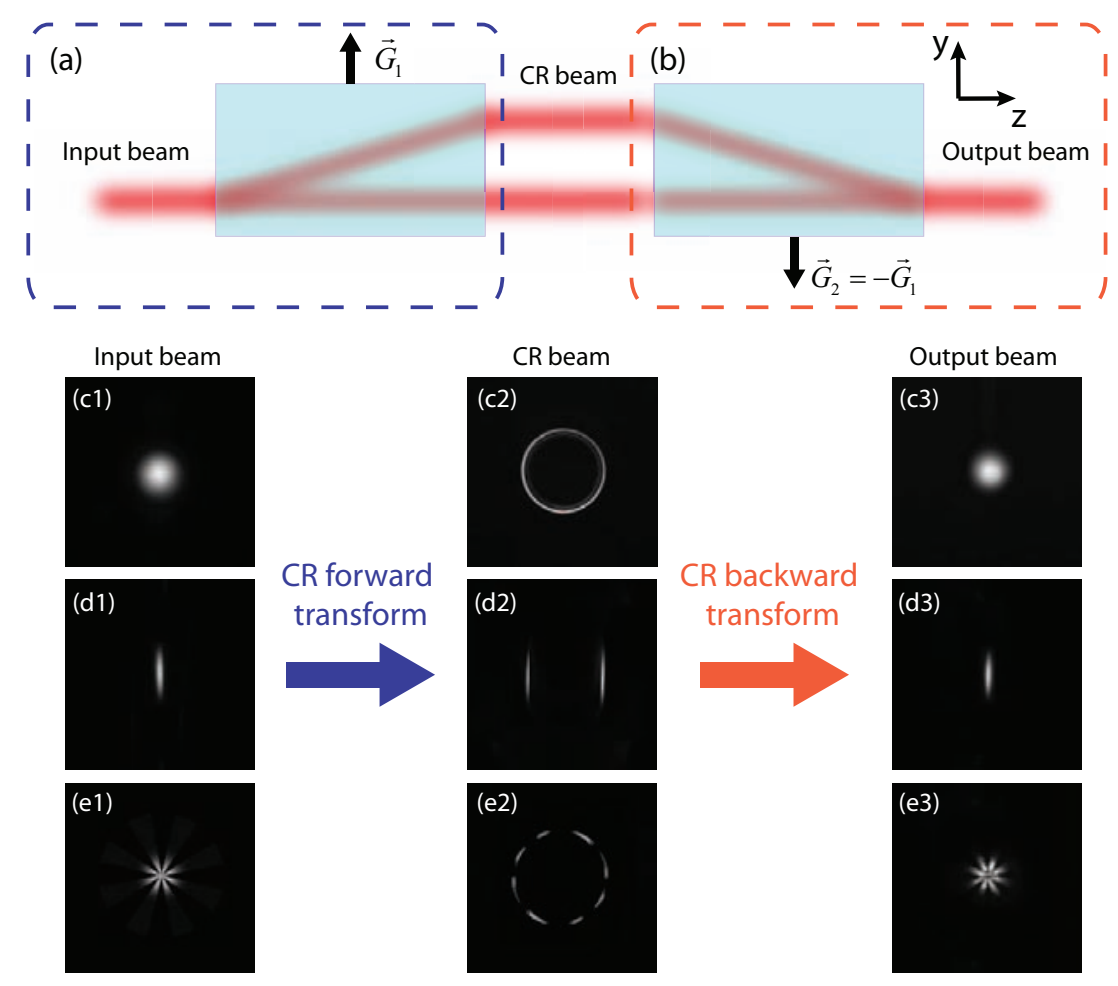

Figure 10.1: Sketch of the forward-backward CR transform provided by a degenerate 2 -crystal cascade configuration with formed by two biaxial crystals with orientation of their characteristic vector $\mathbf{G}$ of (a) $\varphi_{G}=90^{\circ}$ for $\mathbf{G}_{\mathbf{1}}$ and (b) $\varphi_{G}=270^{\circ}$ for $\mathbf{G}_{\mathbf{2}}$. (c1)-(e3) Experimental transverse intensity patterns of the input beam entering into the system (first column), the CR beam obtained at the ring plane (second column) and the output beam emerging from the whole degenerate 2-crystal cascade (third column) for a Gaussian input beam (first row), an elliptical input beam (second row) and a star-shaped input beam (third row).

In Figs. 10.1(c1)-(e3) we show the transverse intensity patterns corresponding to different input beams entering the first biaxial crystal (first column), the CR beam obtained at the ring plane (second column) and the output beam emerging from the whole degenerate 2-crystal cascade (third column) for a Gaussian input beam (first row), an elliptical input beam (second row) and a star-shaped input beam (third row). For a Gaussian input beam, at the ring plane the transverse intensity pattern is formed by the well known pair of concentric bright rings with Poggendorff splitting. As it was already discussed in Sec. 2.3, an elliptical input beam splits into two elliptical beams at the ring plane of the first biaxial crystal. Finally, the star-like input beam is transformed into azimuthal sectors along a ring at the $\mathrm{CR}$ ring plane. As it can be appreciated, the output beams after passing through the degenerate 2-crystal cascade reproduces the input beam. 


\subsection{Multiplexing and de-multiplexing proposal and ex- perimental results}

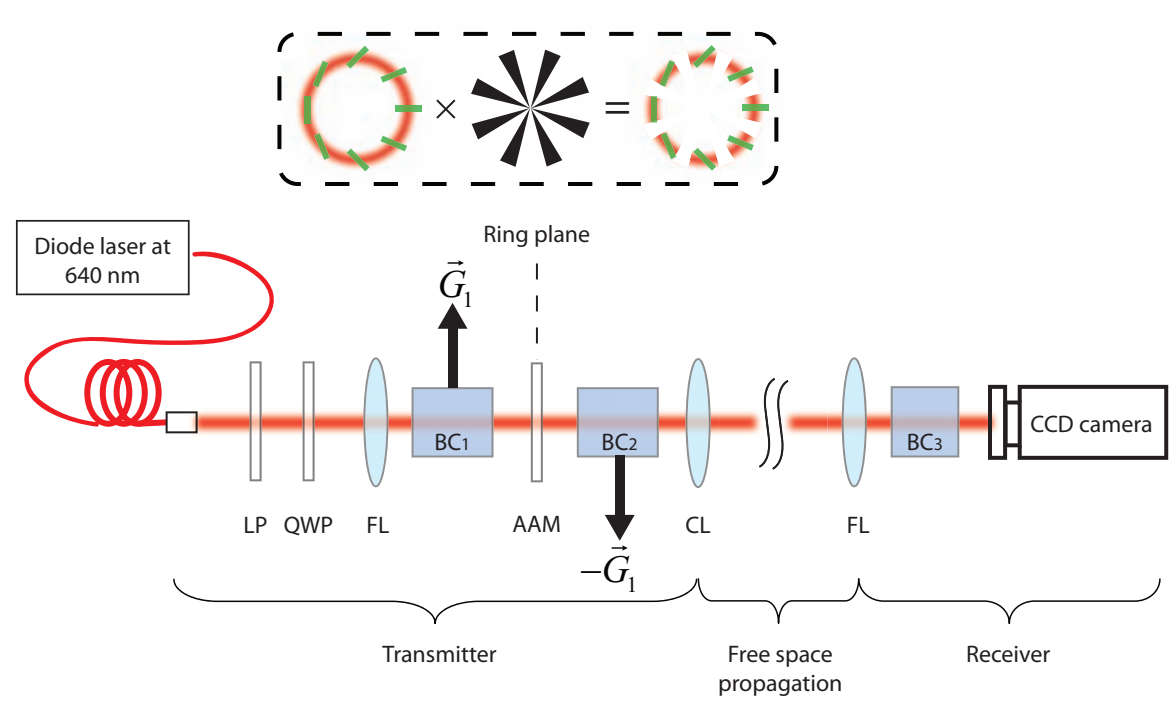

Figure 10.2: Sketch of the polarization de-multiplexing and multiplexing experimental set-up based on CR being used to increase the channel capacity of a FSOC system. The FSOC system is formed by the transmitter with its two identical biaxial crystals presenting opposite $\mathbf{G}$ vectors, a free space propagation distance of $4 \mathrm{~m}$, and the receiver with the third biaxial crystal. The inset shows how polarization signals are selected by using an angular amplitude mask (AAM) that only allows passing some sectors of the CR ring. Green lines indicate the state of polarization. LP: linear polarizer. QWP: quarter wave plate.

In this section, we make use of the forward-backward transform of conical refraction to propose a novel method to de-multiplex and multiplex a monochromatic light beam into a large number of linearly polarized states as it is schematically shown in Fig. 10.2, The first crystal de-multiplexes the input beam into an infinite number of linearly polarized beams placed along a ring (see boxed inset in Fig. 10.2 where each transmitted sector is a linearly polarized beam). Each of these beams constitutes an information channel (note that the channels are polarization channels) that can be individually selected and modulated in amplitude. Later on, the second biaxial crystal multiplexes all the channels into one beam that propagates in free space. Finally, a third biaxial crystal can be used to decode the transmitted signal at the receiver stage. In what follows we describe the $\mathrm{CR}$ de-multiplexing and multiplexing protocol in terms of the standard elements that form a free space optical telecommunications system: the transmitter, the free space propagation, and the receiver. Note that the mutual alignment of the optic axes of the biaxial crystals should be maintained with precision within $50 \mu \mathrm{rad}$ 
to make it work properly by means of the CR effect. In this section, we make use of the forward-backward transform of CR to propose a novel method to de-multiplex and multiplex a monochromatic light beam into a large number of linearly polarized states as it is schematically shown in Fig. 10.2. The first crystal de-multiplexes the input beam into an infinite number of linearly polarized beams placed along a ring (see boxed inset in Fig. 10.2 where each transmitted sector is a linearly polarized beam). Each of these beams constitutes an information channel (note that the channels are polarization channels) that can be individually selected and modulated in amplitude. Later on, the second biaxial crystal multiplexes all the channels into one beam that propagates in free space. Finally, a third biaxial crystal can be used to decode the transmitted signal at the receiver stage. In what follows we describe the CR de-multiplexing and multiplexing protocol in terms of the standard elements that form a free space optical telecommunications system: the transmitter, the free space propagation, and the receiver. Note that the mutual alignment of the optic axes of the biaxial crystals should be maintained with precision within $50 \mu \mathrm{rad}$ to make it work properly by means of the CR effect.

The transmitter consists of an input monochromatic light beam, two biaxial crystals in a degenerate 2-cascade configuration, an angular amplitude mask, and the lenses to focus and collimate the beam. As input beam, we take a collimated linearly polarized Gaussian beam with $w_{0}=1 \mathrm{~mm}$ beam waist obtained from a $640 \mathrm{~nm}$ diode laser coupled to a monomode fiber. A linear polarizer and a quarter wave plate are placed to ensure a perfect circularly polarized Gaussian beam at the entrance of the first crystal. Note that the experiment could also be performed with a linearly polarized input beam but with the inconvenience of producing a crescent intensity pattern instead of a complete ring and, therefore, the polarization channels would possess different amplitudes. The degenerated 2 -cascade scheme is prepared with two identical $\mathrm{KGd}\left(\mathrm{WO}_{4}\right)_{2}$ biaxial crystals $(<100 \mathrm{~nm}$ of difference) yielding a light ring after the first crystal of $872 \mu \mathrm{m}$ ring radius. The polished entrance surfaces of the two biaxial crystals (cross-section $6 \times 4 \mathrm{~mm}^{2}$ ) have parallelism with less than 10 arc seconds and they are perpendicular to one of the two optic crystal axes within $1.5 \mathrm{mrad}$ misalignment angle. To focus and collimate the beam we use lenses with $200 \mathrm{~mm}$ focal length. To select the polarization channels at the light ring we use angular amplitude masks forming a star burst-like pattern with $n$ (up to 12) opened circular sectors, see boxed inset in Fig. 10.2. The amplitude masks actually allow passing only some parts of the ring, thus we are indeed selecting the communication channels. Encoding the information into the different channels could be performed by time varying the transmission coefficient for each sector of the mask using, for instance, a spatial light modulator.

In our experiments, the free space propagation distance is $4 \mathrm{~m}$. We have measured that the multiplexed beam has a divergence similar to the initial Gaussian beam and, therefore, we expect that our protocol could operate for the same distances as other FSOC systems do it with Gaussian beams.

The receiver itself consists of an objective of $50 \mathrm{~mm}$ focal length, a $12 \mathrm{~mm}$ long 
$\mathrm{KGd}\left(\mathrm{WO}_{4}\right)_{2}$ biaxial crystal, and a CCD camera. This biaxial crystal de-multiplexes (final patterns shown in the third row of Fig. 10.3) the free space propagated beam (transverse patterns shown in the second row of Fig.10.3), performing CR and recovering the sectors that were modulated by the angular amplitude masks (first row of Fig. 10.3) at the transmitter. As it can be observed in the third row of Fig. 10.3, we are able to independently modulate up to 12 sectors, which constitutes an increase in one order of magnitude of the channel capacity of the FSOC link. Last row in Fig. 10.3 shows the intensity variation along the azimuthal direction of the corresponding de-multiplexed patterns from the third row of Fig. 10.3. The intensity peaks of the received channels are perfectly distinguishable with respect to the background. Additionally, one can also note that there is no crosstalk between neighbor channels, since there appear as number of peaks as number of channels selected at the transmitter.

(a)

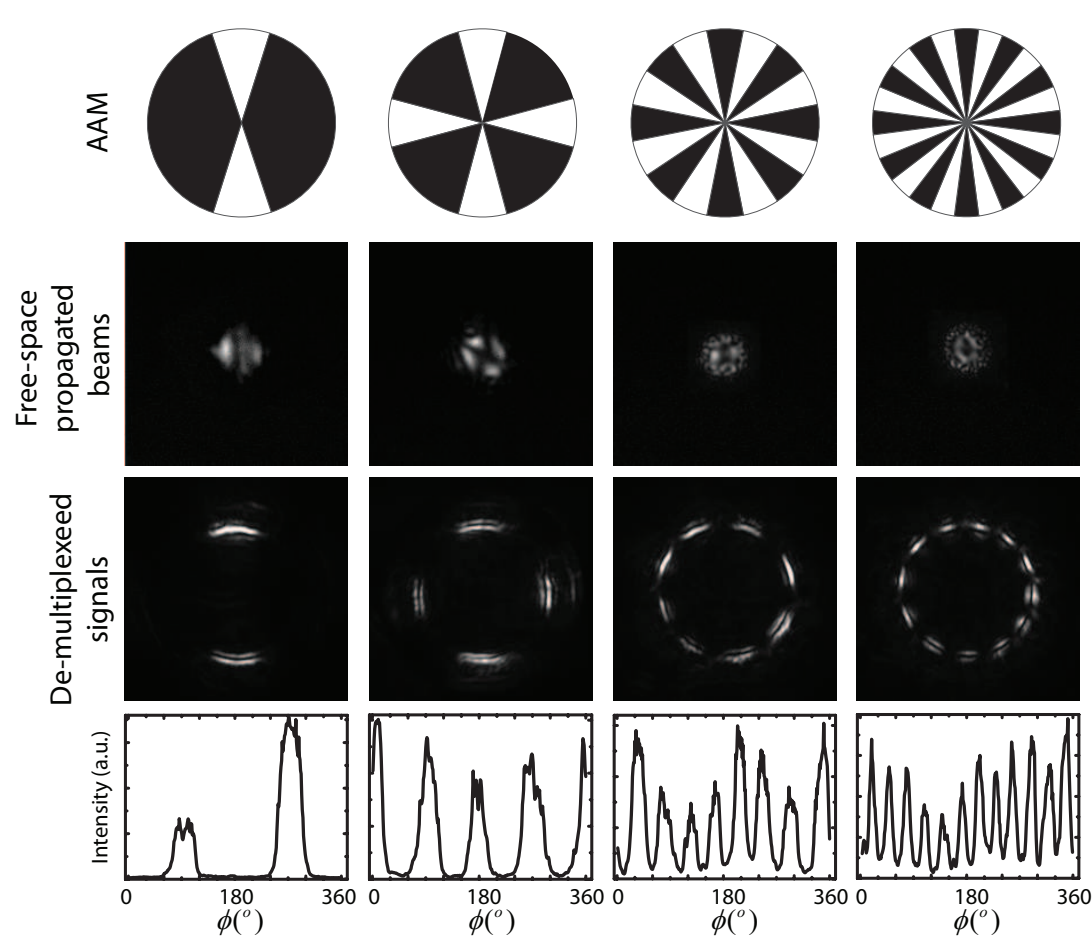

Figure 10.3: Experimental transverse intensity patterns (third row) and the corresponding integrated azimuthal intensities (fourth row) obtained by the receiver for multiplexer masks (first row) with 2 (a), 4 (b), 8 (c), and 12 (d) opened sectors. The second row shows the multiplexed beams at the entrance of the receiver.

Crosstalk (XT) between the channels is one of the main limiting factors for real applications. The main contribution into the XT between adjacent channels in our system comes from light diffraction on the mask domain boundaries. To characterize the XT, we have investigated the influence of the closure angle of the masks, i.e., the 
azimuthal angle separating neighbor open sectors (see $\theta$ in the inset of Fig. 10.4), and the number of channels over it by measuring the residual intensity at the center of the closed sector. For the latter case, the open and closed sectors in the mask have the same azimuthal angular width and we measure the XT at the closed sectors. The results for XT, i.e., residual intensity related to the intensity maximum, are presented in Fig. 10.4. The data reveal that, as it can be expected, the smaller the number of channels the smaller the XT. Moreover, the thinner the open sectors, which corresponds to larger closure angle $\theta$, the smaller the XT too. Red solid curve gives exponential fitting to the experimental data that show the XT decay as $\theta$ increases and $N$ decreases. For the 12 channels case shown in Fig. 10.3, the average XT is less than 3\%. Finally, we would like also to note that misalignment in crystals' rotation around the beam propagation direction leads to light polarization XT between any opposite points at the CR ring. However, in our system the latter is well controlled below $10^{-6}$.

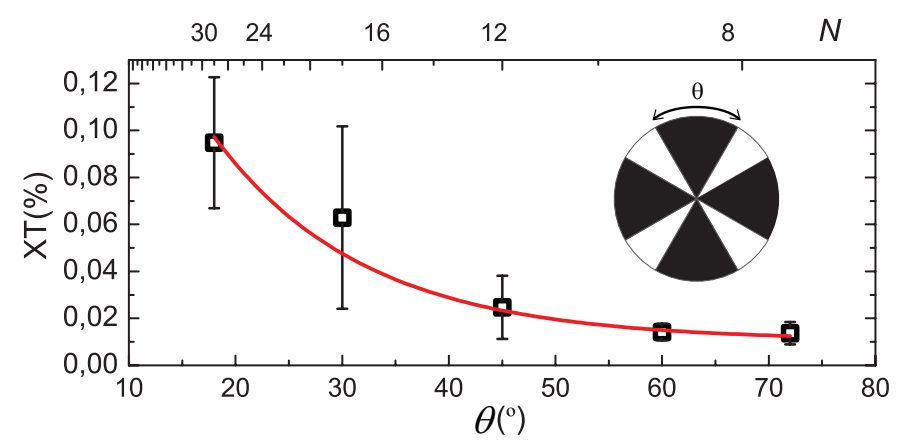

Figure 10.4: Crosstalk (XT) between adjacent channels vs. the closure angle $\theta$ (bottom axis) and vs. the number of channels $N$ (top axis) of the masks used. Red solid curve represents exponential fitting to the experimental data. Uncertainty of the $\theta$ angle measurement was below $1^{\circ}$.

\subsection{Conclusions}

In summary, we have proposed a novel technique to de-multiplex and multiplex a monochromatic light beam into a finite and, in the ideal case, an arbitrary number of linearly polarized states. The technique is based on the forward-backward transform produced by two biaxial crystals under conditions of CR. We have demonstrated an increase of one order of magnitude in the channel capacity for FSOC of a monochromatic input Gaussian beam at $640 \mathrm{~nm}$ for a $4 \mathrm{~m}$ propagation distance with cross-talk being below $3 \%$. In addition, we have investigated the $\mathrm{XT}$ with respect to the azimuthal angle of the closed sectors and the number of sectors of the masks used. The obtained results suggest that by simply optimizing the channel selecting mechanism, i.e., the thickness of the open and closed sectors of the masks, one could increase the channel capacity or decrease the XT for a fixed number of channels. 
As an encouragement for future investigations on the technique proposed in this Chapter, we would like to note that by selecting appropriate biaxial crystals it would be interesting to extend this novel method to other wavelengths in the optical and telecommunication bands at which the crystals are transparent and to combine it with the WDM technique. Finally, it would be very promising to look for new quantum cryptography protocols by extending the technique to the single-photon case. 
CHAPTER 11

Conclusions and outlook

In this last Chapter we aim to discuss and summarize the main contributions of this thesis. Since all Chapters have been summed up at their end, here we will just comment our most relevant contributions.

Within this thesis we have analyzed in detail the phenomenon of conical refraction $(\mathrm{CR})$ occurring in biaxial crystals when a light beam propagates parallel to one of the optic axis and we have applied this phenomenon to different areas of physics such as optical trapping, free-space optical communications or quantum entanglement.

After a brief overview of the phenomenon of $\mathrm{CR}$ and its relevance for the acceptance of the wave theory of light (see Chapter 1), we have exhaustively analyzed the phenomenon of CR both theoretically and experimentally in Chapters 24. For a cylindrically symmetric and homogeneously polarized beam, the diffractive solution of CR (introduced by Belsky and Khapalyuk and reformulated by Berry [26, 27, 32, 74]) is based on two main equations and $\rho_{0} \equiv R_{0} / w_{0}$ is used as the control parameter that structures the output beam, where $R_{0}$ is the CR ring radius under geometrical approximation and $w_{0}$ the waist radius of the focused input beam. We have explored the influence of $\rho_{0}$ over the CR beam and shown that for $\rho_{0} \approx 1$ both the transverse intensity pattern at the focal plane and the evolution of the beam differs from the CR beam found for $\rho_{0} \gg 1$, which is characterized at the focal plane by a pair of concentric bright rings with different intensity split by the Poggendorff dark ring. In particular, we have reported the generation of a super-Gaussian beam [68] and a three-dimensional dark focus for $\rho_{0}=0.45$ and $\rho_{0}=0.92$ [66], respectively.

It is also well known that under conditions of $\rho_{0} \gg 1$ and for a Gaussian input beam the polarization distribution along the CR rings at the focal plane is linear at every point with the azimuth rotating continuously by $\pi \mathrm{rad}$ for a complete turn along the rings. 
In this thesis we have demonstrated that this polarization distribution is only found at the focal plane and that out of the focal plane there exist states possessing non-null ellipticity [69]. Not only this, it has been shown the existence of annular lines forming polarization singularities separating right and left handed circularly polarized states. We have further analyzed the state of polarization of CR beams for $\rho_{0} \approx 1$ and demonstrated the generation of Poincaré beams, i.e., non-homogeneously polarized beams possessing all possible states of polarization. It has been also discussed the generation of optical singularities and we have linked them with the spin-orbit coupling provided by the biaxial crystal. We note here that it would be very interesting to further analyze the spin-orbit coupling and optical singularities generated by non-Gaussian input beams, including non-cylindrically symmetric and non-homogeneously polarized input beams and also for the propagation out of the optic axis.

In this thesis we have also contributed to the diffractive theory of CR by demonstrating that the Hamiltonian operator introduced by Berry [32] can be used to predict the transformation of non-homogeneously polarized and non-cylindrically symmetric beams propagating through a biaxial crystal (or a cascade of them) along any direction [52. The validity of the presented equations has been proved both for Gaussian and elliptical input beams propagating both along the optic axis of a biaxial crystal and out of it and also for a cascade of two biaxial crystals with aligned optic axis.

Although the Belsky-Khapalyuk-Berry equations of CR describes well all the phenomenology associated to CR, they do not offer too much physical intuition about the beam evolution [27,32]. Recently it was proposed that the CR beam can be understood as the interaction of two light cones with slightly different positions of their vertices along the axial direction [59. Our contribution to this picture has been the reformulation of the dual-cone model of $\mathrm{CR}$ and also the experimental demonstration of the existence of two light cones without the need of blocking the beam at any part [56]. A segmented linear polarizer that mimics the usual CR polarization distribution has been used in order to select each of the cones and modify the intensity ratio between the inner and the outer rings of $\mathrm{CR}$.

The last contribution to the theory of $\mathrm{CR}$ reported in the present thesis has been a CR model based in splitting of linearly polarized input waves. In this model, every azimuthal point of the CR ring at the focal plane (Poggendorff splitting is neglected in this approach) is characterized by a transverse wave-vector and a state of linear polarization [54]. When an input wave enters into the crystal, it splits into two subwaves that preserve the original transverse wave-vector and refract to opposite points of the CR ring. The state of linear polarization of these two waves is mutually orthogonal and depends on the orientation of the biaxial crystal. We have shown that, with this simple model, there can be obtained the general features of the final transverse intensity pattern and polarization structure of complex input beams. As a proof of principle, we have applied this formalism to the case of a linearly polarized axicon beam. This theory has been shown to be of significantly usefulness for the calculation of the transverse 
intensity pattern obtained for an arbitrary large cascade of crystals with aligned optic axes [55]. In this case, the resulting pattern after a cascade of $N$ crystals contains in general $2^{N-1}$ concentric bright rings. The intensity of the rings depend on the mutual orientation between the crystals and their radius is a function of the $\mathrm{CR}$ ring radius generated independently by each of the crystals.

The above mentioned splitting of an input wave into two sub-waves makes us glimpse that even if the input beam lacks of some wave-vectors, the CR light ring can still be observed. Following this idea, in Chapter 5 we have also investigated the CR transformation of Gaussian input beams partially blocked by an obstruction [147. We have obtained that the CR phenomenon is a healing-like process that reconstructs partially the output beam emerging from the biaxial crystal, e.g., when half of the input beam is blocked, the transformed intensity pattern still forms a light ring. This has been analyzed for two different scenarios, $\rho_{0} \gg 1$ and $\rho_{0}=0.92$, obtaining that the healing process both in shape and in polarization distribution is more robust for large values of $\rho_{0}$.

When one looks at the shape of CR beams, the first application that is envisaged is optical trapping. While optical trapping is usually associated to optical tweezers, in this thesis we have explored and demonstrated optical trapping of both absorbing particles and Bose-Einstein condensates by using, respectively, the photophoretic force and the dipole force, see Chapters $\mathbf{6}$ and $\mathbf{7}$. On the one hand, it has been demonstrated that the optical bottle formed by a CR beam can be reconfigured by changing the state of polarization of the input beam [142]. In collaboration with the group of Prof. Wieslaw Krolikowski at the Australian National University, we have used this mechanism to efficiently load and unload absorbing glass shells up to $100 \mu \mathrm{m}$ of radius by means of the photophoretic force. In this sense, it would be very promising to combine this technique with the method described in Sec. 2.4 to modulate in real time the intensity distribution between each of the axial intensity maxima of the CR bottle in order to move the particles away from and towards the input source. On the other hand, we have carried out two different implementations of $\mathrm{CR}$ as a dipolar potential for ultra-cold neutral atoms by taking profit of the Poggendorff dark ring and the three-dimensional dark focus reported in Chapter 3. Firstly, a complete characterization of both the Poggendorff dark ring and the three-dimensional dark focus has been made, demonstrating that they form, respectively, a null intensity region surrounded by high intensity walls in three dimensions. For the Poggendorff dark ring case, this implies a dark toroidal potential [178, while the three-dimensional dark focus forms a perfect optical bottle [179]. Then, we have derived the harmonic trapping frequencies and potential depths directly from the CR theory and, in collaboration with the group of Prof. Gerhard Birkl at the Technische Universität Darmstadt, we have experimentally applied these potentials to trap a ${ }^{87} \mathrm{Rb}$ Bose-Einstein condensate. Optical ring potentials are particularly interesting because they represent basic elements in the recently born field of Atomtronics [180,191, 194] and they are ideal candidates for the study of persistent currents and the implementation 
of atomic Sagnac interferometers [270]. To motivate future implementations of novel potentials based on $\mathrm{CR}$, the combination of $\mathrm{CR}$ and microlenses arrays could be used for the generation of bright and dark annular optical lattices. In our case, we have restricted to the case of dark potentials but note that annular bright geometries, including coupled concentric rings, are also available via CR. Note also that reshaping of the CR optical potentials could be possible by modifying in real time the state of polarization of the input beam.

Since both the spatial evolution and the polarization distribution of a CR beam is very rich, in collaboration with Prof. Jose Trull and Prof. Crina Cojocaru from the Universitat Politècnica de Catalunya we have looked at the second harmonic generation (SHG) of this kind of beams in type I and type II non-linear crystals, see Chapter 8 and Ref. [223]. It has been obtained that, in general, when the non-linear crystal is placed at the focal plane of the input CR beam the transverse intensity patterns of the SHG beams resemble the ring-like patterns of the fundamental frequency CR beams but possessing linear polarization at all the points of the light structure. In contrast, when the non-linear crystals are placed out of the focal plane, we have shown that the SHG beams have contributions of both the Raman spot and the CR rings.

The first proposal of combining CR with quantum optics has also been presented in this thesis, in collaboration with the group of Prof. Miles Padgett from the University of Glasgow, see Chapter 9. Firstly, we have analyzed the transformation of a spontaneous parametric down-conversion beam generated in a non-linear crystal, which leads photons entangled in linear momentum. Then, inspired by the wave-vector splitting introduced by the biaxial crystal we have proposed a method to demonstrate such entanglement through a polarization measurement, i.e., we swap linear momentum entanglement into polarization entanglement by means of the conical refraction phenomenon. Unfortunately, the experimental results obtained in Glasgow did not have quality enough to confirm the theoretical proposal.

CR has been also considered for technological applications, being at the heart of a single shot novel polarimeter [131,271] and of a solid state laser [138. In particular, in Chapter 10 of this thesis we have also reported a free space optical communication (FSOC) system for multiplexing and demultiplexing multiple polarization channels with a cascade of 3 biaxial crystals, see Ref. [82] and patent [262. Some sectors of the CR ring generated by the first biaxial crystal are selected by means of amplitude masks and a second biaxial crystal is used to recombine (multiplex) all these sectors back to a single beam. Finally, the third biaxial crystal demultiplexes the selected sectors that form our communication channels. With this system, it has been demonstrated the possibility to increase in one order of magnitude of the channel capacity, i.e., the number of polarization channels, with less than $3 \%$ of crosstalk. This system is interesting since it can work at any wavelength at which the biaxial crystals are transparent. In this case, it would be very interesting to investigate the multiplexing of multiple independent lasers with a single biaxial crystal, i.e., to combine multiple independent lasers. We 
have carried out some investigations in this sense and our preliminary conclusions are that this could only be achieved with linearly polarized elliptical beams. We would like to note that the FSOC system reported in this thesis is not the only technological application of CR. In collaboration with the group of Prof. Edik U. Rafailov at the Aston University (Birmingham), we have shown that the doping elements found in our biaxial crystals can be used to built intracavity lasers with CR patterns [138]. Additionally, due to that for a uniformly polarized input beam there exists a direct link between the intensity distribution of the $\mathrm{CR}$ rings and the state of polarization of the input beam, we have demonstrated and patented a single-shot polarimeter based on CR in collaboration with the group of Prof. Juan Campos at the Universitat Autònoma de Barcelona, see Refs. [131,271,272, for further information.

In conclusion, the applications discussed in this thesis are only some examples of the possibilities to apply CR to different fields of science and technology. Our aim has been to show that the physics behind the phenomenon of CR is very rich and that it can be really useful in a wide variety of situations. We hope that our investigations will motivate the scientific community to take profit of the flexibility offered by the CR phenomenon, giving a second life to this old and almost forgotten optical effect. 

[1] I. Newton. Opticks: or, a treatise of the reflexions, refractions, inflexions and colours of light. Also two treatises of the species and magnitude of curvilinear figures (London, 1704).

[2] R. Bartholin. Experimenta crystalli islandici disdiaclastici: Quibus mira et insolita refractio detegitur Hafniae. (1669).

[3] C. Huygens. Traité de la lumière. (Leiden, 1690).

[4] T. Young. Bakerian lecture: On the theory of light and colours. Phil. Trans. R. Soc. Lond. 92, 12 (1802).

[5] A. Fresnel. Memoir on the Diffraction of Light. (Académie des Sciences, 1819).

[6] M. Born and E. Wolf. Principles of Optics: Electromagnetic Theory of Propagation, Interference and Diffraction of Light. 7th Ed. (London, 1999).

[7] L. D. Landau, E. M. Liftshift, and L. P. Pitaevskii. Electrodynamics of Continuous Media. 2nd Ed. (Oxford, 1984).

[8] H. Lloyd. On the phenomena presented by light in its passage along the axes of biaxal crystals. Philos. Mag. 2, 112 (1833).

[9] W. R. Hamilton. Third supplement to an essay on the theory of systems of rays. Trans. R. Irish Acad. 17, 1 (1837).

[10] J. C. Poggendorff. über die konische refraction. Ann. Phys. Chem. 124, 461 (1839).

[11] R. Potter. An examination of the phenomena of conical refraction in biaxal crystals. Philos. Mag. 159, 194 (1851). 
[12] D. Beerr. Ableitung der intensitäts und polarisations-verhältnisse des lichtringes bei der inneren konischen refraction. Ann. Phys. Chem. 161, 67 (1852).

[13] W. Haidinger. Die konische refraction am diopsid, nebst bemerkungen über einige erscheinungen der konischen refraction am arragonit. Ann. Phys. Chem. 172, 469 (1855).

[14] W. Voigt. Theoretisches und experimentelles zur aufklärung des optischen verhaltens aktiver kristalle. Ann. Phys. Chem. 323, 645 (1905).

[15] C. V. Raman, V. S. Rajagopalan, and T. M. K. Nedungadi. Conical refraction in naphthalene crystals. Nature 147, 268 (1941).

[16] C. V. Raman, V. S. Rajagopalan, and T. M. K. Nedungadi. Conical refraction in naphthalene crystals. Proc. Indian Acad. Sci. 14, 221 (1941).

[17] C. V. Ramani. The phenomena of conical refraction. Curr. Sci. 11, 46 (1942).

[18] R. Goyal and H. Prakash. Conical refraction of light in isotropic nonlinear dielectric in presence of another intense light beam. Phys. Lett. 31, 506 (1970).

[19] D. L. Portigal and E. Burstein. Internal conical refraction. J. Opt. Soc. Am. 59, 1567 (1969).

[20] E. Lalor. The angular spectrum representation of electromagnetic fields in crystals. i. uniaxial crystals. Journal of Mathematical Physics 13 (1972).

[21] E. Lalor. The angular spectrum representation of electromagnetic fields in crystals. ii. biaxial crystals. Journal of Mathematical Physics 13 (1972).

[22] E. Lalor. An analytical approach to the theory of internal conical refraction. Journal of Mathematical Physics 13 (1972).

[23] A. J. Schell and N. Bloembergen. Laser studies of internal conical diffraction. $i$. quantitative comparison of experimental and theoretical conical intensity distribution in aragonite. J. Opt. Soc. Am. 68, 1093 (1978).

[24] B. S. Perkal'skis and Y. P. Mikhailichenko. Demonstration of conical refraction. Sov. Phys. J. 22, 901 (1979).

[25] T. S. Velichkina, O. I. Vasil'eva, A. I. Israilenko, and I. A. Yakovlev. Demonstration of phenomena of conical refraction. Usp. Fiz. Nauk 130, 357 (1980).

[26] A. M. Belskii and A. P. Khapalyuk. Propagation of confined light beams along the beam axes (axes of single ray velocity) of biaxial crystals. Opt. Spectrosc. 44, 312 (1978).

[27] A. M. Belskii and A. P. Khapalyuk. Internal conical refraction of bounded light beams in biaxial crystals. Opt. Spectrosc. 44, 436 (1978). 
[28] J. P. Féve, B. Boulanger, and G. Marnier. Experimental study of internal and external conical refractions in ktp. Opt. Commun. 105, 243 (1994).

[29] A. M. Belsky and M. A. Stepanov. Internal conical refraction of coherent light beams. Opt. Commun. 167, 1 (1999).

[30] A. M. Belsky and M. A. Stepanov. Internal conical refraction of light beams in biaxial gyrotropic crystals. Opt. Commun. 204, 1 (2002).

[31] A. Belafhal. Theoretical intensity distribution of internal conical refraction. Optics Communications 178, 257 (2000).

[32] M. V. Berry. Conical diffraction asymptotics: fine structure of poggendorff rings and axial spike. Journal of Optics A: Pure and Applied Optics 6, 289 (2004).

[33] T. K. Kalkandjiev and M. A. Bursukova. Conical refraction: an experimental introduction (2008).

[34] M. J. P. Musgrave (San Francisco).

[35] T. P. Srinivasant and G. Lakshmi. Internal conical refraction of elastic waves in tetragonal crystals. J. Phys. D: Appl. Phys. 6, 306 (1973).

[36] V. I. Al'shits, A. N. Darinskii, and A. L. Shuvalov. Disclinations in a vector field of the polarizations of acoustic and optical beams under conical refraction conditions. Zh. Eksp. Teor. Fiz. 96, 1958 (1989).

[37] P. Boulanger and M. Hayes. Acoustic axes for elastic waves in crystals: theory and applications. Proc. R. Soc. Lond. A 454, 2323 (1998).

[38] B. D. Zaitsev and I. E. Kuznetsova. Behavior of acoustic axes and internal conical refraction in linbo 3 and srtio 3 crystals placed in an external electric field. IEEE Trans. Ultrason. Ferroelec. Freq. Contr. 45, 361 (1998).

[39] M. O. I. Skab, I. I. Martynyuk-Lototska, and R. Vlokh. Generation of helical acoustic modes using anisotropic crystals. Ukr. J. Phys. Opt. 12, 47 (2011).

[40] V. I. Alshits and V. N. Lyubimov. Conical refraction of elastic waves in absorbing crystals. J. Exp. Theor. Phys. 113, 659 (2011).

[41] J. de Klerk and M. J. P. Musgrave. Internal conical refraction of transverse elastic waves in a cubic crystal. Proc. Phys. Soc. B68, 81 (1955).

[42] H. J. McSkimin and W. L. Bond. Conical refraction of transverse ultrasonic waves in quartz. J. Acoust. Soc. Am. 39, 499 (1966).

[43] R. A. Artman. Ultrasonic internal conical refraction in potassium chloride. J. Acoust. Soc. Am. 39, 493 (1966). 
[44] D. Tisklauri. On the conical refraction of hydromagnetic waves in plasma with anisotropic thermal pressure. Phys. Plasmas 3, 800 (1996).

[45] B. E. Applegate, T. A. Barckholtz, and A. M. Miller. Explorations of conical intersections and their ramifications for chemistry through the jahn-teller effecte. Chem. Soc. Rev. 32, 38 (2003).

[46] M. J. Ablowitz, S. D. Nixon, and Y. Zhu. Conical diffraction in honeycomb lattices. Phys. Rev. A 79, 053830 (2009).

[47] H. Ramezani, T. Kottos, V. Kovanis, and D. N. Christodoulides. Exceptionalpoint dynamics in photonic honeycomb lattices with pt symmetry. Phys. Rev. A 85, 013818 (2012).

[48] D. Leykam, O. Bahat-Treidel, and A. S. Desyatnikov. Conical diffraction, pseudospin, and nonlinear wave dynamics in photonic lieb lattices. CLEO EUROPE/IQEC (2013).

[49] O. Peleg, G. Bartal, B. Freedman, O. Manela, M. Segev, and D. N. Christodoulides. Conical diffraction and gap solitons in honeycomb photonic lattices. Phys. Rev. Lett. 98, 103901 (2007).

[50] A. A. Bulgakov and I. V. Fedorin. The phenomenon of conical refraction in a thinlayer periodic semiconductor-dielectric structure in a magnetic field. Opt. Spectr. 112, 474 (2012).

[51] S. Matos, C. Paiva, and A. Barbosa. Conical refraction in generalized biaxial media: A geometric algebra approach. In EUROCON - International Conference on Computer as a Tool (EUROCON), 2011 IEEE, 1-3 (2011).

[52] A. Turpin, Y. V. Loiko, T. K. Kalkandjiev, and J. Mompart. Light propagation in biaxial crystals. Journal of Optics 17, 065603 (2015).

[53] A. Turpin, A. Vargas, A. Lizana, F. A. Torres-Ruiz, I. Estévez, I. Moreno, J. Campos, and J. Mompart. Transformation of vector beams with radial and azimuthal polarizations in biaxial crystals. J. Opt. Soc. Am. A 32, 1012 (2015).

[54] A. Turpin, Y. V. Loiko, T. K. Kalkandjiev, H. Tomizawa, and J. Mompart. Wavevector and polarization dependence of conical refraction. Opt. Express 21, 4503 (2013).

[55] A. Turpin, Y. V. Loiko, T. K. Kalkandjiev, and J. Mompart. Multiple rings formation in cascaded conical refraction. Opt. Lett. 38, 1455 (2013).

[56] A. Turpin, Y. Loiko, T. K. Kalkandjiev, H. Tomizawa, and J. Mompart. On the dual-cone nature of the conical refraction phenomenon. Opt. Lett. 40, 1639 (2015). 
[57] M. A. Dreger. Optical beam propagation in biaxial crystals. Journal of Optics A: Pure and Applied Optics 1, 601 (1999).

[58] J. Garnier. High-frequency asymptotics for maxwell equations in anisotropic media part i: Linear geometric and diffractive optics. Journal of Mathematical Physics 42 (2001).

[59] G. S. Sokolovskii, D. J. Carnegie, T. K. Kalkandjiev, and E. U. Rafailov. Conical refraction: New observations and a dual cone model. Opt. Express 21, 11125 (2013).

[60] M. Pujol, M. Rico, C. Zaldo, R. Solé, V. Nikolov, X. Solans, M. Aguiló, and F. Díaz. Crystalline structure and optical spectroscopy of er ${ }^{3+}$-doped $k g d\left(w_{4}\right)_{2}$ single crystals. Applied Physics B 68, 187 (1999).

[61] M. Berry, M. Jeffrey, and J. Lunney. Conical diffraction: observations and theory. Proceedings of the Royal Society of London A: Mathematical, Physical and Engineering Sciences 462, 1629 (2006).

[62] C. F. Phelan, D. P. O’Dwyer, Y. P. Rakovich, J. F. Donegan, and J. G. Lunney. Conical diffraction and bessel beam formation with a high optical quality biaxial crystal. Opt. Express 17, 12891 (2009).

[63] V. Peet. Improving directivity of laser beams byemploying the effect of conical refractionin biaxial crystals. Opt. Express 18, 19566 (2010).

[64] V. Peet and D. Zolotukhin. Free-space evolution of focused gaussian beams transformed by conical diffraction in a biaxial crystal. Optics Communications 283, 3011 (2010).

[65] V. Peet. The far-field structure of gaussian light beams transformed by internal conical refraction in a biaxial crystal. Optics Communications 311, 150 (2013).

[66] Y. V. Loiko, A. Turpin, T. K. Kalkandjiev, E. U. Rafailov, and J. Mompart. Generating a three-dimensional dark focus from a single conically refracted light beam. Opt. Lett. 38, 4648 (2013).

[67] S. D. Grant and A. Abdolvand. Evolution of conically diffracted gaussian beams in free space. Opt. Express 22, 3880 (2014).

[68] A. Turpin, Y. V. Loiko, T. K. Kalkandkiev, H. Tomizawa, and J. Mompart. Supergaussian conical refraction beam. Opt. Lett. 39, 4349 (2014).

[69] A. Turpin, Y. V. Loiko, A. Peinado, A. Lizana, T. K. Kalkandjiev, J. Campos, and J. Mompart. Polarization tailored novel vector beams based on conical refraction. Opt. Express 23, 5704 (2015). 
[70] V. Peet. Conical refraction and formation of multiring focal image with laguerregauss light beams. Opt. Lett. 36, 2913 (2011).

[71] V. Peet. Experimental study of internal conical refraction in a biaxial crystal with laguerre-gauss light beams. Journal of Optics 16, 075702 (2014).

[72] R. T. Darcy, D. McCloskey, K. E. Ballantine, J. G. Lunney, P. R. Eastham, and J. F. Donegan. Conical diffraction intensity profiles generated using a top-hat input beam. Opt. Express 22, 11290 (2014).

[73] Y. V. Loiko, M. A. Bursukova, T. K. Kalkanjiev, E. U. Rafailov, and J. Mompart. Fermionic transformation rules for spatially filtered light beams in conical refraction (2011).

[74] M. Berry and M. Jeffrey. Chapter 2 conical diffraction: Hamilton's diabolical point at the heart of crystal optics. volume 50 of Progress in Optics, 13 - 50 (Elsevier, 2007).

[75] C. F. Phelan, K. E. Ballantine, P. R. Eastham, J. F. Donegan, and J. G. Lunney. Conical diffraction of a gaussian beam with a two crystal cascade. Opt. Express 20, 13201 (2012).

[76] V. Peet. Biaxial crystal as a versatile mode converter. Journal of Optics 12, 095706 (2010).

[77] S. D. Grant and A. Abdolvand. Left- and right-circularly polarized light in cascade conical diffraction. Opt. Lett. 37, 5226 (2012).

[78] A. Abdolvand. Conical diffraction from a multi-crystal cascade: experimental observations. Applied Physics B 103, 281 (2011).

[79] K. Wilcox, A. Abdolvand, T. Kalkandjiev, and E. Rafailov. Laser with simultaneous gaussian and conical refraction outputs. Applied Physics B 99, 619 (2010).

[80] D. P. O’Dwyer, K. E. Ballantine, C. F. Phelan, J. G. Lunney, and J. F. Donegan. Optical trapping using cascade conical refraction of light. Opt. Express 20, 21119 (2012).

[81] D. P. O'Dwyer, C. F. Phelan, Y. P. Rakovich, P. R. Eastham, J. G. Lunney, and J. F. Donegan. The creation and annihilation of optical vortices using cascade conical diffraction. Opt. Express 19, 2580 (2011).

[82] A. Turpin, Y. Loiko, T. K. Kalkandjiev, and J. Mompart. Free-space optical polarization demultiplexing and multiplexing by means of conical refraction. Opt. Lett. 37, 4197 (2012).

[83] M. V. Berry. Conical diffraction from an n-crystal cascade. Journal of Optics 12, 075704 (2010). 
[84] J. Hellström, H. Henricsson, V. Pasiskevicius, U. Bünting, and D. Haussmann. Polarization-tunable yb:kgw laser based on internal conical refraction. Opt. Lett. 32, 2783 (2007).

[85] S. Rosen, G. Y. Sirat, H. Ilan, and A. J. Agranat. A sub wavelength localization scheme in optical imaging using conical diffraction. Opt. Express 21, 10133 (2013).

[86] V. G. Shvedov, A. V. Rode, Y. V. Izdebskaya, A. S. Desyatnikov, W. Krolikowski, and Y. S. Kivshar. Giant optical manipulation. Phys. Rev. Lett. 105, 118103 (2010).

[87] C. J. R. Sheppard and A. Choudhury. Annular pupils, radial polarization, and superresolution. Appl. Opt. 43, 4322 (2004).

[88] A. Bouhelier, F. Ignatovich, A. Bruyant, C. Huang, G. C. des Francs, J.-C. Weeber, A. Dereux, G. P. Wiederrecht, and L. Novotny. Surface plasmon interference excited by tightly focused laser beams. Opt. Lett. 32, 2535 (2007).

[89] M. Meier, V. Romano, and T. Feurer. Material processing with pulsed radially and azimuthally polarized laser radiation. Applied Physics A 86, 329 (2007).

[90] L. Allen, M. W. Beijersbergen, R. J. C. Spreeuw, and J. P. Woerdman. Orbital angular momentum of light and the transformation of laguerre-gaussian laser modes. Phys. Rev. A 45, 8185 (1992).

[91] M. Berry and M. Dennis. Polarization singularities in isotropic random vector waves. Proceedings of the Royal Society of London A: Mathematical, Physical and Engineering Sciences 457, 141 (2001).

[92] J. Leach, M. R. Dennis, J. Courtial, and M. J. Padgett. Laser beams: knotted threads of darkness. Nature 432, 165 (2004).

[93] W. T. Irvine and D. Bouwmeester. Linked and knotted beams of light. Nature Physics 4, 716 (2008).

[94] M. R. Dennis, R. P. King, B. Jack, K. O'Holleran, and M. J. Padgett. Isolated optical vortex knots. Nature Physics 6, 118 (2010).

[95] J. Arlt and M. J. Padgett. Generation of a beam with a dark focus surrounded by regions of higher intensity: the optical bottle beam. Opt. Lett. 25, 191 (2000).

[96] N. Davidson, H. Jin Lee, C. S. Adams, M. Kasevich, and S. Chu. Long atomic coherence times in an optical dipole trap. Phys. Rev. Lett. 74, 1311 (1995).

[97] Cacciapuoti, L., de Angelis, M., Pierattini, G., and Tino, G. M. Single-beam optical bottle for cold atoms using a conical lens. Eur. Phys. J. D 14, 373 (2001). 
[98] T. Kuga, Y. Torii, N. Shiokawa, T. Hirano, Y. Shimizu, and H. Sasada. Novel optical trap of atoms with a doughnut beam. Phys. Rev. Lett. 78, 4713 (1997).

[99] L. Isenhower, W. Williams, A. Dally, and M. Saffman. Atom trapping in an interferometrically generated bottle beam trap. Opt. Lett. 34, 1159 (2009).

[100] V. G. Shvedov, C. Hnatovsky, N. Shostka, and W. Krolikowski. Generation of vector bottle beams with a uniaxial crystal. JOSA B 30, 1 (2013).

[101] Q. Zhan. Cylindrical vector beams: from mathematical concepts to applications. Adv. Opt. Photon. 1, 1 (2009).

[102] K. Bongs, S. Burger, S. Dettmer, D. Hellweg, J. Arlt, W. Ertmer, and K. Sengstock. Waveguide for bose-einstein condensates. Phys. Rev. A 63, 031602 (2001).

[103] V. Bagini, D. Ambrosini, G. S. Spagnolo, R. Borghi, F. Gori, A. M. Pacileo, and M. Santarsiero. Propagation of axially symmetric flattened gaussian beams. J. Opt. Soc. Am. A 13, 1385 (1996).

[104] I. Gur and D. Mendlovic. Diffraction limited domain flat-top generator. Optics Communications 145, 237 (1998).

[105] A. A. Tovar. Propagation of flat-topped multi-gaussian laser beams. J. Opt. Soc. Am. A 18, 1897 (2001).

[106] Y. Cai and S. He. Partially coherent flattened gaussian beam and its paraxial propagation properties. J. Opt. Soc. Am. A 23, 2623 (2006).

[107] Y. Dan and B. Zhang. Beam propagation factor of partially coherent flat-topped beams in a turbulent atmosphere. Opt. Express 16, 15563 (2008).

[108] F. Wang and Y. Cai. Experimental generation of a partially coherent flat-topped beam. Opt. Lett. 33, 1795 (2008).

[109] D. Liu and Z. Zhou. Propagation of partially coherent flat-topped beams in uniaxial crystals orthogonal to the optical axis. J. Opt. Soc. Am. A 26, 924 (2009).

[110] I. A. Litvin and A. Forbes. Intra-cavity flat-top beam generation. Opt. Express 17, 15891 (2009).

[111] H. Ma, Z. Liu, P. Zhou, X. Wang, Y. Ma, and X. Xu. Generation of flat-top beam with phase-only liquid crystal spatial light modulators. Journal of Optics $\mathbf{1 2}$, 045704 (2010).

[112] K. Jahn and N. Bokor. Intensity control of the focal spot by vectorial beam shaping. Optics Communications 283, 4859 (2010).

[113] W. Han, W. Cheng, and Q. Zhan. Flattop focusing with full poincaré beams under low numerical aperture illumination. Opt. Lett. 36, 1605 (2011). 
[114] A. Hendriks, D. Naidoo, F. S. Roux, C. López-Mariscal, and A. Forbes. The generation of flat-top beams by complex amplitude modulation with a phase-only spatial light modulator (2012).

[115] S. Ngcobo, K. Ait-Ameur, I. Litvin, A. Hasnaoui, and A. Forbes. Tuneable gaussian to flat-top resonator by amplitude beam shaping. Opt. Express 21, 21113 (2013).

[116] H. Chen, S. Tripathi, and K. C. Toussaint. Demonstration of flat-top focusing under radial polarization illumination. Opt. Lett. 39, 834 (2014).

[117] R. E. Grojean, D. Feldman, and J. F. Roach. Production of flat top beam profiles for high energy lasers. Review of Scientific Instruments 51 (1980).

[118] W. Wang, P. X. Wang, Y. K. Ho, Q. Kong, Y. Gu, and S. J. Wang. Vacuum electron acceleration and bunch compression by a flat-top laser beam. Review of Scientific Instruments 78, 093103 (2007).

[119] C. Maher-McWilliams, P. Douglas, and P. F. Barker. Laser-driven acceleration of neutral particles. Nature Photonics 6, 386 (2012).

[120] C. Zhao, Y. Cai, X. Lu, and H. T. Eyyuboğlu. Radiation force of coherent and partially coherent flat-topped beams on a rayleigh particle. Opt. Express 17, 1753 (2009).

[121] S. Gras, D. G. Blair, and L. Ju. Opto-acoustic interactions in gravitational wave detectors: Comparing flat-top beams with gaussian beams. Phys. Rev. D 81, 042001 (2010).

[122] J. W. Kim, J. I. Mackenzie, J. R. Hayes, and W. A. Clarkson. High-power er:yag laser with quasi-top-hat output beam. Opt. Lett. 37, 1463 (2012).

[123] F. M. Dickey and S. C. Holswade. Laser Beam Shaping: Theory and Techniques (London, 2000).

[124] D. Maluenda, I. Juvells, R. Martínez-Herrero, and A. Carnicer. Reconfigurable beams with arbitrary polarization and shape distributions at a given plane. Opt. Express 21, 5432 (2013).

[125] X.-L. Wang, J. Ding, W.-J. Ni, C.-S. Guo, and H.-T. Wang. Generation of arbitrary vector beams with a spatial light modulator and a common path interferometric arrangement. Opt. Lett. 32, 3549 (2007).

[126] A. Desyatnikov, T. A. Fadeyeva, V. G. Shvedov, Y. V. Izdebskaya, A. V. Volyar, E. Brasselet, D. N. Neshev, W. Krolikowski, and Y. S. Kivshar. Spatially engineered polarization states and optical vortices in uniaxial crystals. Opt. Express 18, 10848 (2010). 
[127] J. F. Nye and M. V. Berry. Dislocations in wave trains. Proceedings of the Royal Society of London A: Mathematical, Physical and Engineering Sciences 336, 165 (1974).

[128] M. R. Dennis, K. O'Holleran, and M. J. Padgett. Chapter 5 singular optics: Optical vortices and polarization singularities. volume 53 of Progress in Optics, 293 - 363 (Elsevier, 2009).

[129] D. Kleckner and W. T. Irvine. Creation and dynamics of knotted vortices. Nature Physics 9, 253 (2013).

[130] I. Freund. Ordinary polarization singularities in three-dimensional optical fields. Opt. Lett. 37, 2223 (2012).

[131] A. Peinado, A. Turpin, A. Lizana, E. Fernández, J. Mompart, and J. Campos. Conical refraction as a tool for polarization metrology. Opt. Lett. 38, 4100 (2013).

[132] L. Allen, M. W. Beijersbergen, R. J. C. Spreeuw, and J. P. Woerdman. Orbital angular momentum of light and the transformation of laguerre-gaussian laser modes. Phys. Rev. A 45, 8185 (1992).

[133] G. Molina-Terriza, J. P. Torres, and L. Torner. Twisted photons. Nature Physics 3, 305 (2007).

[134] J. P. Torres and L. Torner. Twisted photons: applications of light with orbital angular momentum (John Wiley \& Sons, 2011).

[135] A. M. Yao and M. J. Padgett. Orbital angular momentum: origins, behavior and applications. Adv. Opt. Photon. 3, 161 (2011).

[136] R. W. Schoonover and T. D. Visser. Polarization singularities of focused, radially polarized fields. Opt. Express 14, 5733 (2006).

[137] V. Shvedov, P. Karpinski, Y. Sheng, X. Chen, W. Zhu, W. Krolikowski, and C. Hnatovsky. Visualizing polarization singularities in bessel-poincar\&\#x00e9; beams. Opt. Express 23, 12444 (2015).

[138] Y. V. Loiko, G. S. Sokolovskii, D. Carnegie, A. Turpin, J. Mompart, and E. U. Rafailov. Laser beams with conical refraction patterns. In SPIE LASE, 89601Q89601Q (International Society for Optics and Photonics, 2014).

[139] R. W. Boyd. Intuitive explanation of the phase anomaly of focused light beams. J. Opt. Soc. Am. 70, 877 (1980).

[140] M. V. Berry, M. R. Jeffrey, and M. Mansuripur. Orbital and spin angular momentum in conical diffraction. Journal of Optics A: Pure and Applied Optics 7, 685 (2005). 
[141] A. M. Beckley, T. G. Brown, and M. A. Alonso. Full poincare beams. Opt. Express 18, 10777 (2010).

[142] A. Turpin, V. Shvedov, C. Hnatovsky, Y. V. Loiko, J. Mompart, and W. Krolikowski. Optical vault: A reconfigurable bottle beam based on conical refraction of light. Opt. Express 21, 26335 (2013).

[143] V. Shvedov, A. R. Davoyan, C. Hnatovsky, N. Engheta, and W. Krolikowski. A long-range polarization-controlled optical tractor beam. Nature Photonics $\mathbf{8}, 846$ (2014).

[144] Y. Loiko, V. Ahufinger, R. Menchon-Enrich, G. Birkl, and J. Mompart. Coherent injecting, extracting, and velocity filtering of neutral atoms in a ring trap via spatial adiabatic passage. The European Physical Journal D 68, 147 (2014).

[145] G. Gariepy, J. Leach, K. T. Kim, T. J. Hammond, E. Frumker, R. W. Boyd, and P. B. Corkum. Creating high-harmonic beams with controlled orbital angular momentum. Phys. Rev. Lett. 113, 153901 (2014).

[146] R. Fickler, R. Lapkiewicz, S. Ramelow, and A. Zeilinger. Quantum entanglement of complex photon polarization patterns in vector beams. Phys. Rev. A 89, 060301 (2014).

[147] A. Turpin, Y. V. Loiko, T. K. Kalkandjiev, R. Corbalán, and J. Mompart. Conical refraction healing after partially blocking the input beam. Phys. Rev. A 92, 013802 (2015).

[148] J. Durnin, J. J. Miceli, and J. H. Eberly. Diffraction-free beams. Phys. Rev. Lett. 58, 1499 (1987).

[149] S. Vyas, Y. Kozawa, and S. Sato. Self-healing of tightly focused scalar and vector bessel-gauss beams at the focal plane. J. Opt. Soc. Am. A 28, 837 (2011).

[150] G. Wu, F. Wang, and Y. Cai. Generation and self-healing of a radially polarized bessel-gauss beam. Phys. Rev. A 89, 043807 (2014).

[151] G. A. Siviloglou, J. Broky, A. Dogariu, and D. N. Christodoulides. Observation of accelerating airy beams. Phys. Rev. Lett. 99, 213901 (2007).

[152] J. Broky, G. A. Siviloglou, A. Dogariu, and D. N. Christodoulides. Self-healing properties of optical airy beams. Opt. Express 16, 12880 (2008).

[153] J. D. Ring, J. Lindberg, A. Mourka, M. Mazilu, K. Dholakia, and M. R. Dennis. Auto-focusing and self-healing of pearcey beams. Opt. Express 20, 18955 (2012).

[154] N. Hermosa, C. Rosales-Guzmán, and J. P. Torres. Helico-conical optical beams self-heal. Opt. Lett. 38, 383 (2013). 
[155] P. Zhang, Y. Hu, T. Li, D. Cannan, X. Yin, R. Morandotti, Z. Chen, and X. Zhang. Nonparaxial mathieu and weber accelerating beams. Phys. Rev. Lett. 109, 193901 (2012).

[156] V. Garces-Chavez, D. McGloin, H. Melville, W. Sibbett, and K. Dholakia. Simultaneous micromanipulation in multiple planes using a self-reconstructing light beam. Nature 419, 145 (2002).

[157] J. Baumgartl, M. Mazilu, and K. Dholakia. Optically mediated particle clearing using airy wavepackets. Nature photonics 2, 675 (2008).

[158] F. O. Fahrbach, P. Simon, and A. Rohrbach. Microscopy with self-reconstructing beams. Nature photonics 4, 780 (2010).

[159] N. S. Kazak, N. A. Khilo, and A. A. Ryzhevich. Generation of bessel light beams under the conditions of internal conical refraction. Quantum Electronics 29, 1020 (1999).

[160] T. King, W. Hogervorst, N. Kazak, N. Khilo, and A. Ryzhevich. Formation of higher-order bessel light beams in biaxial crystals. Optics Communications 187, 407 (2001).

[161] Á. Turpin and J. Mompart. Una cámara acorazada óptica. Investigación y ciencia 14-15 (2015).

[162] A. Ashkin. Optical trapping and manipulation of neutral particles using lasers. Proceedings of the National Academy of Sciences 94, 4853 (1997).

[163] D. G. Grier. A revolution in optical manipulation. Nature 424, 810 (2003).

[164] K. Dholakia, P. Reece, and M. Gu. Optical micromanipulation. Chem. Soc. Rev. 37, 42 (2008).

[165] M. Woerdemann, C. Alpmann, M. Esseling, and C. Denz. Advanced optical trapping by complex beam shaping. Laser \& Photonics Reviews 7, 839 (2013).

[166] K. T. Gahagan and G. A. Swartzlander. Optical vortex trapping of particles. Opt. Lett. 21, 827 (1996).

[167] T. Čižmár, M. Mazilu, and K. Dholakia. In situ wavefront correction and its application to micromanipulation. Nature Photonics 4, 388 (2010).

[168] D. McGloin, G. Spalding, H. Melville, W. Sibbett, and K. Dholakia. Applications of spatial light modulators in atom optics. Opt. Express 11, 158 (2003).

[169] V. G. Shvedov, A. V. Rode, Y. V. Izdebskaya, A. S. Desyatnikov, W. Krolikowski, and Y. S. Kivshar. Selective trapping of multiple particles by volume speckle field. Optics express 18, 3137 (2010). 
[170] S. E. Olson, M. L. Terraciano, M. Bashkansky, and F. K. Fatemi. Cold-atom confinement in an all-optical dark ring trap. Phys. Rev. A 76, 061404 (2007).

[171] P. Xu, X. He, J. Wang, and M. Zhan. Trapping a single atom in a blue detuned optical bottle beam trap. Opt. Lett. 35, 2164 (2010).

[172] A. S. Desyatnikov, V. G. Shvedov, A. V. Rode, W. Krolikowski, and Y. S. Kivshar. Photophoretic manipulation of absorbing aerosol particles with vortex beams: theory versus experiment. Opt. Express 17, 8201 (2009).

[173] V. G. Shvedov, C. Hnatovsky, A. V. Rode, and W. Krolikowski. Robust trapping and manipulation of airborne particles with a bottle beam. Opt. Express 19, 17350 (2011).

[174] O. Jovanovic. Photophoresis: Light induced motion of particles suspended in gas. Journal of Quantitative Spectroscopy and Radiative Transfer 110, 889 (2009). Light Scattering: Mie and More Commemorating 100 years of Mie's 1908 publication.

[175] B. Redding, S. C. Hill, D. Alexson, C. Wang, and Y.-L. Pan. Photophoretic trapping of airborne particles using ultraviolet illumination. Opt. Express 23, 3630 (2015).

[176] C. Wang, Y.-L. Pan, S. C. Hill, and B. Redding. Photophoretic trapping-raman spectroscopy for single pollens and fungal spores trapped in air. Journal of Quantitative Spectroscopy and Radiative Transfer 153, 4 (2015). Topical issue on optical particle characterization and remote sensing of the atmosphere: Part $\{\mathrm{II}\}$.

[177] V. G. Shvedov, C. Hnatovsky, N. Eckerskorn, A. V. Rode, and W. Krolikowski. Polarization-sensitive photophoresis. Applied Physics Letters 101, 051106 (2012).

[178] A. Turpin, J. Polo, Y. V. Loiko, J. Küber, F. Schmaltz, T. K. Kalkandjiev, V. Ahufinger, G. Birkl, and J. Mompart. Blue-detuned optical ring trap for boseeinstein condensates based on conical refraction. Opt. Express 23, 1638 (2015).

[179] A. Turpin, , J. Küber, F. Schmaltz, J. Mompart, and G. Birkl. Submitted to publication .

[180] B. T. Seaman, M. Krämer, D. Z. Anderson, and M. J. Holland. Atomtronics: Ultracold-atom analogs of electronic devices. Phys. Rev. A 75, 023615 (2007).

[181] M. K. Olsen and A. S. Bradley. Quantum ultracold atomtronics. Phys. Rev. A 91, 043635 (2015).

[182] N. Lundblad, M. Schlosser, and J. V. Porto. Experimental observation of magicwavelength behavior of ${ }^{87} \mathrm{Rb}$ atoms in an optical lattice. Phys. Rev. A 81, 031611 (2010). 
[183] S. Zhang, F. Robicheaux, and M. Saffman. Magic-wavelength optical traps for rydberg atoms. Phys. Rev. A 84, 043408 (2011).

[184] M. Takamoto, H. Katori, S. I. Marmo, V. D. Ovsiannikov, and V. G. Pal'chikov. Prospects for optical clocks with a blue-detuned lattice. Phys. Rev. Lett. 102, 063002 (2009).

[185] C. Monroe. Quantum information processing with atoms and photons. Nature 416, 238 (2002).

[186] A. L. Gaunt, T. F. Schmidutz, I. Gotlibovych, R. P. Smith, and Z. Hadzibabic. Bose-einstein condensation of atoms in a uniform potential. Phys. Rev. Lett. 110, 200406 (2013).

[187] P. Rudy, R. Ejnisman, A. Rahman, S. Lee, and N. P. Bigelow. An all optical dynamical dark trap for neutral atoms. Opt. Express 8, 159 (2001).

[188] G. Li, S. Zhang, L. Isenhower, K. Maller, and M. Saffman. Crossed vortex bottle beam trap for single-atom qubits. Opt. Lett. 37, 851 (2012).

[189] R. Grimm, M. Weidemüller, and Y. B. Ovchinnikov. Optical dipole traps for neutral atoms. volume 42 of Advances In Atomic, Molecular, and Optical Physics, 95 - 170 (Academic Press, 2000).

[190] J. Küber. Dynamics of Bose-Einstein condensates in novel optical potentials (Technische Universität Darmstadt, Darmstadt, 2014).

[191] R. A. Pepino, J. Cooper, D. Z. Anderson, and M. J. Holland. Atomtronic circuits of diodes and transistors. Phys. Rev. Lett. 103, 140405 (2009).

[192] A. Ruschhaupt and J. G. Muga. Atom diode: A laser device for a unidirectional transmission of ground-state atoms. Phys. Rev. A 70, 061604 (2004).

[193] J. J. Thorn, E. A. Schoene, T. Li, and D. A. Steck. Experimental realization of an optical one-way barrier for neutral atoms. Phys. Rev. Lett. 100, 240407 (2008).

[194] J. A. Stickney, D. Z. Anderson, and A. A. Zozulya. Transistorlike behavior of a bose-einstein condensate in a triple-well potential. Phys. Rev. A 75, 013608 (2007).

[195] A. D. Cronin, J. Schmiedmayer, and D. E. Pritchard. Optics and interferometry with atoms and molecules. Rev. Mod. Phys. 81, 1051 (2009).

[196] D. M. Stamper-Kurn, M. R. Andrews, A. P. Chikkatur, S. Inouye, H.-J. Miesner, J. Stenger, and W. Ketterle. Optical confinement of a bose-einstein condensate. Phys. Rev. Lett. 80, 2027 (1998). 
[197] M. D. Barrett, J. A. Sauer, and M. S. Chapman. All-optical formation of an atomic bose-einstein condensate. Phys. Rev. Lett. 87, 010404 (2001).

[198] R. Ozeri, L. Khaykovich, and N. Davidson. Long spin relaxation times in a singlebeam blue-detuned optical trap. Phys. Rev. A 59, R1750 (1999).

[199] E. M. Wright, J. Arlt, and K. Dholakia. Toroidal optical dipole traps for atomic bose-einstein condensates using laguerre-gaussian beams. Phys. Rev. A 63, 013608 (2000).

[200] G. Birkl, F. Buchkremer, R. Dumke, and W. Ertmer. Atom optics with microfabricated optical elements. Optics Communications 191, 67 (2001).

[201] T. Müther, J. Nes, A.-L. Gehrmann, M. Volk, W. Ertmer, G. Birkl, M. Gruber, and J. Jahns. Atomic quantum systems in optical micro-structures. Journal of Physics: Conference Series 19, 97 (2005).

[202] S. K. Schnelle, E. D. van Ooijen, M. J. Davis, N. R. Heckenberg, and H. Rubinsztein-Dunlop. Versatile two-dimensional potentials for ultra-cold atoms. Opt. Express 16, 1405 (2008).

[203] N. Houston, E. Riis, and A. S. Arnold. Reproducible dynamic dark ring lattices for ultracold atoms. Journal of Physics B: Atomic, Molecular and Optical Physics 41, 211001 (2008).

[204] S. Franke-Arnold, J. Leach, M. J. Padgett, V. E. Lembessis, D. Ellinas, A. J. Wright, J. M. Girkin, P. Ohberg, and A. S. Arnold. Optical ferris wheel for ultracold atoms. Opt. Express 15, 8619 (2007).

[205] T. Freegarde and K. Dholakia. Cavity-enhanced toroidal dipole force traps for dark-field seeking species. Optics Communications 201, 99 (2002).

[206] E. Courtade, O. Houde, J.-F. m. c. Clément, P. Verkerk, and D. Hennequin. Dark optical lattice of ring traps for cold atoms. Phys. Rev. A 74, 031403 (2006).

[207] C. Ryu, M. F. Andersen, P. Cladé, V. Natarajan, K. Helmerson, and W. D. Phillips. Observation of persistent flow of a bose-einstein condensate in a toroidal trap. Phys. Rev. Lett. 99, 260401 (2007).

[208] A. Ramanathan, K. C. Wright, S. R. Muniz, M. Zelan, W. T. Hill, C. J. Lobb, K. Helmerson, W. D. Phillips, and G. K. Campbell. Superflow in a toroidal boseeinstein condensate: An atom circuit with a tunable weak link. Phys. Rev. Lett. 106, 130401 (2011).

[209] A. I. Yakimenko, Y. M. Bidasyuk, O. O. Prikhodko, S. I. Vilchinskii, E. A. Ostrovskaya, and Y. S. Kivshar. Optical tweezers for vortex rings in bose-einstein condensates. Phys. Rev. A 88, 043637 (2013). 
[210] L. Corman, L. Chomaz, T. Bienaimé, R. Desbuquois, C. Weitenberg, S. Nascimbène, J. Dalibard, and J. Beugnon. Quench-induced supercurrents in an annular bose gas. Phys. Rev. Lett. 113, 135302 (2014).

[211] J. G. Lee and W. T. Hill. Spatial shaping for generating arbitrary optical dipole traps for ultracold degenerate gases. Review of Scientific Instruments 85, 103106 (2014).

[212] S. Eckel, J. G. Lee, F. Jendrzejewski, N. Murray, C. W. Clark, C. J. Lobb, W. D. Phillips, M. Edwards, and G. K. Campbell. Hysteresis in a quantized superfluid atomtronic circuit. Nature 506, 200 (2014).

[213] T. Lauber, J. Küber, O. Wille, and G. Birkl. Optimized bose-einstein-condensate production in a dipole trap based on a 1070-nm multifrequency laser: Influence of enhanced two-body loss on the evaporation process. Phys. Rev. A 84, 043641 (2011).

[214] R. T. Darcy, D. McCloskey, K. E. Ballantine, B. D. Jennings, J. G. Lunney, P. R. Eastham, and J. F. Donegan. White light conical diffraction. Opt. Express 21, 20394 (2013).

[215] M. Schlosser, S. Tichelmann, J. Kruse, and G. Birkl. Scalable architecture for quantum information processing with atoms in optical micro-structures. Quantum Information Processing 10, 907 (2011).

[216] K. C. Wright, R. B. Blakestad, C. J. Lobb, W. D. Phillips, and G. K. Campbell. Driving phase slips in a superfluid atom circuit with a rotating weak link. Phys. Rev. Lett. 110, 025302 (2013).

[217] C. Ryu, P. W. Blackburn, A. A. Blinova, and M. G. Boshier. Experimental realization of josephson junctions for an atom squid. Phys. Rev. Lett. 111, 205301 (2013).

[218] C. Ryu, K. C. Henderson, and M. G. Boshier. Creation of matter wave bessel beams and observation of quantized circulation in a bose-einstein condensate. New Journal of Physics 16, 013046 (2014).

[219] L. Amico, A. Osterloh, and F. Cataliotti. Quantum many particle systems in ring-shaped optical lattices. Phys. Rev. Lett. 95, 063201 (2005).

[220] A. S. Maciej Lewenstein and V. Ahufinger. Ultracold Atoms in Optical Lattices: Simulating quantum many-body systems (Oxford, 2012).

[221] O. Morizot, Y. Colombe, V. Lorent, H. Perrin, and B. M. Garraway. Ring trap for ultracold atoms. Phys. Rev. A 74, 023617 (2006). 
[222] D. Aghamalyan, L. Amico, and L. C. Kwek. Effective dynamics of cold atoms flowing in two ring-shaped optical potentials with tunable tunneling. Phys. Rev. A 88, 063627 (2013).

[223] A. Turpin, Y. V. Loiko, T. K. Kalkandjiev, J. Trull, C. Cojocaru, and J. Mompart. Type $i$ and type ii second harmonic generation of conically refracted beams. Opt. Lett. 38, 2484 (2013).

[224] H. Shih and N. Bloembergen. Conical refraction in second-harmonic generation. Phys. Rev. 184, 895 (1969).

[225] A. J. Schell and N. Bloembergen. Laser studies of internal conical diffraction. iii. second-harmonic conical refraction in $\alpha$-iodic acid. Phys. Rev. A 18, 2592 (1978).

[226] V. Stroganov, A. Illarionov, and B. Kidyarov. Conical refraction in second harmonic generation in a crystal of lithium formate. Journal of Applied Spectroscopy 32, 341 (1980).

[227] T. S. Velichkina, O. I. Vasil'eva, A. I. Israilenko, and I. A. Yakovlev. Demonstration of phenomena of conical refraction. Soviet Physics Uspekhi 23, 176 (1980).

[228] J. Kroupa. Second-harmonic conical refraction in guhp. Journal of Optics 12 , 045706 (2010).

[229] S. Zolotovskaya, A. Abdolvand, T. Kalkandjiev, and E. Rafailov. Second-harmonic conical refraction: observation of free and forced harmonic waves. Applied Physics B 103, 9 (2011).

[230] V. Peet and S. Shchemelyov. Frequency doubling with laser beams transformed by conical refraction in a biaxial crystal. Journal of Optics 13, 055205 (2011).

[231] S. D. Grant, S. A. Zolotovskaya, T. K. Kalkandjiev, W. A. Gillespie, and A. Abdolvand. On the frequency-doubled conically-refracted gaussian beam. Opt. Express 22, 21347 (2014).

[232] R. W. Boyd. Nonlinear optics (Academic press, 2003).

[233] A. Einstein, B. Podolsky, and N. Rosen. Can quantum-mechanical description of physical reality be considered complete? Phys. Rev. 47, 777 (1935).

[234] N. Bohr. Can quantum-mechanical description of physical reality be considered complete? Phys. Rev. 48, 696 (1935).

[235] J. S. Bell et al. On the einstein-podolsky-rosen paradox. Physics 1, 195 (1964).

[236] A. Aspect. Bell's theorem: The naive view of an experimentalist. arXiv:quant$\mathrm{ph} / 0402001$ (2004). 
[237] S. J. Freedman and J. F. Clauser. Experimental test of local hidden-variable theories. Phys. Rev. Lett. 28, 938 (1972).

[238] F. M. Pipkin. Atomic Physics Tests of the Basic Concepts in Quantum Mechanics. Advances in Atomic and Molecular Physics 14, 281 (1979).

[239] J. F. Clauser. Experimental investigation of a polarization correlation anomaly. Phys. Rev. Lett. 36, 1223 (1976).

[240] S. J. Freedman and J. F. Clauser. Experimental test of local hidden-variable theories. Phys. Rev. Lett. 28, 938 (1972).

[241] A. Aspect, P. Grangier, and G. Roger. Experimental tests of realistic local theories via bell's theorem. Phys. Rev. Lett. 47, 460 (1981).

[242] A. Aspect, P. Grangier, and G. Roger. Experimental realization of einsteinpodolsky-rosen-bohm Gedankenexperiment : A new violation of bell's inequalities. Phys. Rev. Lett. 49, 91 (1982).

[243] A. Aspect, J. Dalibard, and G. Roger. Experimental test of bell's inequalities using time- varying analyzers. Phys. Rev. Lett. 49, 1804 (1982).

[244] P. G. Kwiat, K. Mattle, H. Weinfurter, A. Zeilinger, A. V. Sergienko, and Y. Shih. New high-intensity source of polarization-entangled photon pairs. Physical Review Letters 75, 4337 (1995).

[245] J. Brendel, E. Mohler, and W. Martienssen. Experimental test of bell's inequality for energy and time. EPL (Europhysics Letters) 20, 575 (1992).

[246] W. Tittel, J. Brendel, T. Herzog, H. Zbinden, and N. Gisin. Non-local two-photon correlations using interferometers physically separated by 35 meters. EPL (Europhysics Letters) 40, 595 (1997).

[247] M. Hendrych, M. Micuda, and J. P. Torres. Tunable control of the frequency correlations of entangled photons. Opt. Lett. 32, 2339 (2007).

[248] C. I. Osorio, G. Molina-Terriza, B. G. Font, and J. P. Torres. Azimuthal distinguishability of entangled photons generated in spontaneous parametric downconversion. Opt. Express 15, 14636 (2007).

[249] R. Fickler, R. Lapkiewicz, M. Huber, M. P. Lavery, M. J. Padgett, and A. Zeilinger. Interface between path and orbital angular momentum entanglement for highdimensional photonic quantum information. Nature Communications 5, 4502 (2014).

[250] A. Mair, A. Vaziri, G. Weihs, and A. Zeilinger. Entanglement of the orbital angular momentum states of photons. Nature 412, 313 (2001). 
[251] G. Molina-Terriza, J. P. Torres, and L. Torner. Orbital angular momentum of photons in noncollinear parametric downconversion. Optics Communications 228, 155 (2003).

[252] J. P. Torres, Y. Deyanova, L. Torner, and G. Molina-Terriza. Preparation of engineered two-photon entangled states for multidimensional quantum information. Phys. Rev. A 67, 052313 (2003).

[253] C. I. Osorio, G. Molina-Terriza, and J. P. Torres. Correlations in orbital angular momentum of spatially entangled paired photons generated in parametric downconversion. Phys. Rev. A 77, 015810 (2008).

[254] J. Leach, B. Jack, J. Romero, M. Ritsch-Marte, R. Boyd, A. Jha, S. Barnett, S. Franke-Arnold, and M. Padgett. Violation of a bell inequality in twodimensional orbital angular momentum state-spaces. Optics express $\mathbf{1 7}, 8287$ (2009).

[255] B. Jack, J. Leach, J. Romero, S. Franke-Arnold, M. Ritsch-Marte, S. M. Barnett, and M. J. Padgett. Holographic ghost imaging and the violation of a bell inequality. Phys. Rev. Lett. 103, 083602 (2009).

[256] D. A. Kleinman. Theory of optical parametric noise. Phys. Rev. 174, 1027 (1968).

[257] Y. H. Shih and C. O. Alley. New type of einstein-podolsky-rosen-bohm experiment using pairs of light quanta produced by optical parametric down conversion. Phys. Rev. Lett. 61, 2921 (1988).

[258] I. Ali Khan and J. C. Howell. Experimental demonstration of high two-photon time-energy entanglement. Phys. Rev. A 73, 031801 (2006).

[259] J. C. Howell, R. S. Bennink, S. J. Bentley, and R. W. Boyd. Realization of the einstein-podolsky-rosen paradox using momentum- and position-entangled photons from spontaneous parametric down conversion. Phys. Rev. Lett. 92, 210403 (2004).

[260] P.-A. Moreau, F. Devaux, and E. Lantz. Einstein-podolsky-rosen paradox in twin images. Phys. Rev. Lett. 113, 160401 (2014).

[261] J. Romero, D. Giovannini, S. Franke-Arnold, S. M. Barnett, and M. J. Padgett. Increasing the dimension in high-dimensional two-photon orbital angular momentum entanglement. Phys. Rev. A 86, 012334 (2012).

[262] A. Turpin, Y. V. Loiko, T. K. Kalkandjiev, and J. Mompart. System, method, transmitter and receptor for optical communications (2012). EP Patent P201230105.

[263] B. Mukherjee. Wdm optical communication networks: progress and challenges. Selected Areas in Communications, IEEE Journal on 18, 1810 (2000). 
[264] G. Gibson, J. Courtial, M. J. Padgett, M. Vasnetsov, V. Pas'ko, S. M. Barnett, and S. Franke-Arnold. Free-space information transfer using light beams carrying orbital angular momentum. Optics Express 12, 5448 (2004).

[265] J. Wang, J.-Y. Yang, I. M. Fazal, N. Ahmed, Y. Yan, H. Huang, Y. Ren, Y. Yue, S. Dolinar, M. Tur, et al. Terabit free-space data transmission employing orbital angular momentum multiplexing. Nature Photonics 6, 488 (2012).

[266] J. Torres. Optical communications: Multiplexing twisted light. Nature Photonics 6, 420 (2012).

[267] M. Krenn, R. Fickler, M. Fink, J. Handsteiner, M. Malik, T. Scheidl, R. Ursin, and A. Zeilinger. Communication with spatially modulated light through turbulent air across vienna. New Journal of Physics 16, 113028 (2014).

[268] B. Jack, M. J. Padgett, and S. Franke-Arnold. Angular diffraction. New Journal of Physics 10, 103013 (2008).

[269] S. Franke-Arnold, S. M. Barnett, E. Yao, J. Leach, J. Courtial, and M. Padgett. Uncertainty principle for angular position and angular momentum. New Journal of Physics 6, 103 (2004).

[270] J. L. Helm, S. L. Cornish, and S. A. Gardiner. Sagnac interferometry using bright matter-wave solitons. Phys. Rev. Lett. 114, 134101 (2015).

[271] A. Peinado, A. Lizana, A. Turpin, C. Iemmi, T. K. Kalkandjiev, J. Mompart, and J. Campos. Optimization, tolerance analysis and implementation of a stokes polarimeter based on the conical refraction phenomenon. Optics Express 23, 5636 (2015).

[272] A. Turpin, A. Peinado, A. Lizana, T. K. Kalkandjiev, J. Campos, and J. Mompart. Complete polarimeter based on conical refraction (2014). EP Patent PCT/EP2014/065,918. 